\title{
Neural network representability of fully ionized plasma fluid model closures
}

\author{
Romit Maulik, ${ }^{1}$ Nathan A. Garland, ${ }^{2,}$ a) Joshua W. Burby, ${ }^{2}$ Xian-Zhu Tang, ${ }^{2}$ and \\ Prasanna Balaprakash ${ }^{3,1}$ \\ 1) Argonne Leadership Computing Facility, Argonne National Laboratory, Lemont, \\ IL 60439, USA \\ ${ }^{2)}$ Theoretical Division, Los Alamos National Laboratory, Los Alamos, NM 87545, \\ USA \\ ${ }^{3)}$ Mathematics and Computer Science Division, Argonne National Laboratory, Lemont, \\ IL 60439, USA
}

(Dated: June 22, 2020)

The closure problem in fluid modeling is a well-known challenge to modelers aiming to accurately describe their system of interest. Over many years, analytic formulations in a wide range of regimes have been presented but a practical, generalized fluid closure for magnetized plasmas remains an elusive goal. In this study, as a first step towards constructing a novel data-based approach to this problem, we apply ever-maturing machine learning methods to assess the capability of neural network architectures to reproduce crucial physics inherent in known magnetized plasma closures. We find encouraging results, indicating the applicability of neural networks to closure physics, but also arrive at recommendations on how one should choose appropriate network architectures for given locality properties dictated by underlying physics of the plasma.

\footnotetext{
a)Electronic mail: ngarland@lanl.gov
} 


\section{INTRODUCTION}

In modeling and simulation of plasma dynamics many practitioners employ a fluid, or moment, model. ${ }^{1-3}$ The attraction of a fluid model lies in the generally simpler model description, and far-reduced computational cost, when compared to high fidelity kinetic models that describe a charged particle distribution function, $f(\boldsymbol{r}, \boldsymbol{v}, t)$. To determine which of these two approaches to take, one must assess the trade-off between an averaged macroscopic description of a plasma that is computationally tractable, versus a fine kinetic microscopic description that may carry extreme computational cost. In the event of employing a fluid model, one must sufficiently truncate or close the infinite hierarchy of equations in the velocity moments of $f(\boldsymbol{r}, \boldsymbol{v}, t)$.

While truncation at higher order may be done, it is more common to enforce some underlying physical properties of the problem by deriving a non-trivial closure for higher-order moments as functions of lower-order moments, e.g. heat flux as a function of density, velocity and energy. The physical properties used to derive closures can range from collisional to collisionless, and can enforce local or non-local space-time relationships within the plasma ${ }^{4-6}$. This being said, there are some scenarios where complex physics prevents a simple closure being assumed, and the question as to what closure to employ has a non-trivial answer. In this situation, some research communities, particularly fluid mechanics in recent years, ${ }^{7-9}$ have turned to machine learning to try to construct surrogate closure models that map the known macroscopic variables in a fluid model to the higher order moments that must be closed .

Machine and deep learning methods have long been used in fusion and plasma physics applications. ${ }^{10-14}$ In this study we seek to follow in the footsteps of the fluid mechanics community by using machine learning to formulate and study surrogate models in a proof-of-principle study for magnetized plasma closure models that are crucial to fluid modeling. We note recent work of Ma et al. ${ }^{15}$ on the analysis of closure surrogates via neural networks for the Hammett-Perkins closure $^{16}$. In this work, not only will we touch on the Hammett-Perkins non-local closure but also expand to examine additional closures in different regimes relevant to fusion applications. ${ }^{2,17}$ These closures were specifically chosen to provide separate regime challenges for machine learning surrogates, with the goal of understanding the implications of a broader range of closure physics on potential machine learning methods and architectures.

The primary goal of this work is to verify the ability of neural networks to accurately represent several known closure functions that apply in different physics regimes. These limiting regimes are 
(1) collisional limit, (2) collisionless limit with periodic boundary condition, and (3) collisionless limit with an upstream Maxwellian source and a downstream absorbing wall. Ultimately we would like to learn closure surrogates in the intermediate collisionality regime, with complex source and boundary conditions, but these would likely be produced by data-driven learning from high fidelity kinetic simulations where more complex physics may occur than that described by known analytic closures in the limiting regimes examined here. We believe an important first step towards producing such practical closures using machine learning is to perform a benchmark study to explore whether some of the physics we value, contained in the simpler analytic closures used in our study, can be described well by neural networks and, if so, what styles of architecture are preferable for certain types of physics. This will help us make more intelligent decisions on neural network properties in order to capture necessary physics found in more general problems.

In seeking to learn surrogate closure models we aim to provide a priori physical intuition for making wise network design choices that reduce the computational complexity of the learning task. We contend that benchmarking learned surrogates against well known analytic closures in important physical limits, and understanding the structure and limitations of the neural networks that formulate closure surrogates, is an instructive exercise. In this study we will show that, in the important physical limits grounding the closures studied, there is benefit in tailoring a network's architecture to the physics regime the closure is designed for. This should be contrasted with the "black-box" approach of employing neural networks that have proven to be useful in unrelated contexts. The lessons learned from this study will inform future development of closure surrogates for practical application in more physically-complex scenarios.

Our methodology and results are presented as follows. In Section II we briefly review fluid models for magnetized plasmas and some of the closure methods used. Section III outlines the learning method employed to formulate the closure surrogate neural networks, with Section IV detailing the network architectures employed for each surrogate. The results obtained from training the appropriate networks on each benchmark closure are discussed in Section V followed by a summary of the key findings of this work in Section VI.

\section{PLASMA FLUID MODELING}

When considering electron dynamics in a plasma, the fundamental description of electrons is carried by the electron distribution function, $f_{e}(\boldsymbol{r}, \boldsymbol{v}, t)$, in phase space. While many techniques 
exist to resolve $f_{e}(\boldsymbol{r}, \boldsymbol{v}, t)$, the computational load can be enormous. The primary approach is solution of the kinetic equation for $f_{e}(\boldsymbol{r}, \boldsymbol{v}, t)$

$$
\frac{\partial f_{e}}{\partial t}+\boldsymbol{v} \cdot \frac{\partial f_{e}}{\partial \boldsymbol{x}}+\frac{q}{m_{e}}(\boldsymbol{E}+\boldsymbol{v} \times \boldsymbol{B}) \cdot \frac{\partial f_{e}}{\partial \boldsymbol{v}}=C\left(f_{e}\right)
$$

where $C\left(f_{e}\right)$ is the collision operator.

As an alternative, a fluid description of the plasma is a popular approach that can simulate plasma properties in a more computationally efficient manner. Macroscopic fluid quantities, such as density $n_{e}$, mean velocity $\boldsymbol{u}_{e}$, and pressure $\mathrm{P}_{e}$ are obtained via velocity moments of $f_{e}(\boldsymbol{r}, \boldsymbol{v}, t)$

$$
\begin{gathered}
n_{e}(\boldsymbol{r}, t)=\int f_{e}(\boldsymbol{r}, \boldsymbol{v}, t) d^{3} \boldsymbol{v} \\
\boldsymbol{u}_{e}(\boldsymbol{r}, t)=\frac{1}{n_{e}(\boldsymbol{r}, t)} \int \boldsymbol{v} f_{e}(\boldsymbol{r}, \boldsymbol{v}, t) d^{3} \boldsymbol{v} \\
\mathrm{P}_{e}(\boldsymbol{r}, t)=m_{e} \int \boldsymbol{w} \boldsymbol{w} f_{e}(\boldsymbol{r}, \boldsymbol{v}, t) d^{3} \boldsymbol{v}
\end{gathered}
$$

where $\boldsymbol{w}=\boldsymbol{v}-\boldsymbol{u}_{e}$ is the fluctuation velocity, $m_{e}$ is the electron mass, and the scalar pressure $p_{e}$ can be extracted from the pressure tensor via $\mathrm{P}_{e}=p_{e} \mathrm{I}+\Pi_{\mathrm{e}}$, where $\mathrm{I}$ is the identity tensor and $\Pi_{\mathrm{e}}$ is the viscosity tensor.

While fluid modeling is an attractive option to simulate plasmas, there are inherent challenges and disadvantages that this technique brings with it. Fundamentally, lack of exact knowledge of the velocity space distribution of electrons leads to an inherent inaccuracy in modeling discharges far from equilibrium, where a Maxwellian distribution function is often assumed. An extension of not knowing the velocity space distribution is the closure problem, where higher-order moments, such as the viscosity tensor, $\Pi_{e}$, or heat flux, $\boldsymbol{q}_{e}$, must be approximated using lower-order moments to enable computation.

\section{A. Collisional limit: Braginskii closure}

In his seminal paper, ${ }^{2}$ Braginskii tackled the problem of showing convincingly that hydrodynamics reigns in a plasma made up of electrons and a single ion species moving through a strong magnetic field, where collisions occur at a rate that is comparable to the gyrofrequency. The wellknown Braginskii ${ }^{2}$ equations for electron transport, dropping the species subscript for $n, \boldsymbol{u}$, and $p$, 
can be written

$$
\begin{gathered}
\frac{d n}{d t}+n \nabla \cdot \boldsymbol{u}=0, \\
m_{e} n \frac{d \boldsymbol{u}}{d t}+\nabla p+\nabla \cdot \Pi+e n(\boldsymbol{E}+\boldsymbol{u} \times \boldsymbol{B})=\boldsymbol{F}, \\
\frac{3}{2} \frac{d p}{d t}+\frac{5}{2} p \nabla \cdot \boldsymbol{u}+\Pi: \nabla \boldsymbol{u}+\nabla \cdot \boldsymbol{q}=W,
\end{gathered}
$$

where $\frac{d}{d t}=\frac{\partial}{\partial t}+\boldsymbol{u} \cdot \nabla$ is the commonly used convective or material derivative, $\Pi$ is the viscosity tensor, $\boldsymbol{q}$ is the heat flux vector, $\boldsymbol{F}$ is a frictional force due to plasma resistivity and thermal forces, and $W$ is an energy transfer function due to collisions with ions and work done by frictional forces.

Braginskii showed, through an asymptotic solution of the kinetic equation underlying equations (5) - (7), that one can estimate the heat flux vector $\boldsymbol{q}$, the viscosity tensor $\Pi$, the frictional force vector $\boldsymbol{F}$, and the energy exchange function $W$ in terms of the hydrodynamic observables.

In this study we seek to identify a network architecture that is capable of representing Braginskii's approximation to the closure given a range of macroscopic input profiles. To that end, a suitable litmus test is to assess the representability of one characteristic piece of the closure functional, namely the so-called gyroviscous stress tensor

$$
\Pi^{\mathrm{BG}}(n, \boldsymbol{u}, T)(\boldsymbol{x})=\frac{n(\boldsymbol{x}) T(\boldsymbol{x})}{4\|\boldsymbol{B}(\boldsymbol{x})\|}\left(\tilde{\Pi}+\tilde{\Pi}^{T}\right),
$$

where

$$
\tilde{\Pi}=\hat{b}(\boldsymbol{x}) \times\left(\nabla \boldsymbol{u}(\boldsymbol{x})+\nabla \boldsymbol{u}^{T}(\boldsymbol{x})\right) \cdot(\boldsymbol{I}+3 \hat{\boldsymbol{b}}(\boldsymbol{x}) \hat{\boldsymbol{b}}(\boldsymbol{x}))
$$

$\hat{\boldsymbol{b}}=\boldsymbol{B} /\|\boldsymbol{B}\|$ is the magnetic field unit vector, and I is the $3 \times 3$ identity tensor.

The Braginskii formulation provides one benchmark limit for learned neural network architectures that could provide a surrogate closure model in the collisional magnetized plasma limit, with a local functional dependence. In contrast to this, the following sections will describe non-local closures in collisionless plasma closure limits.

\section{B. Collisionless limit: Hammett-Perkins closure}

Linear Landau damping is an inherently kinetic effect in weakly-collisional plasmas wherein fluid moments of the single-particle distribution function decay due to the development of increasingly-fine-scale structure in velocity space. These small-scale features are produced as 
a result of higher-velocity parts of the distribution function being transported by the streaming effect more rapidly than lower-velocity parts. A similar effect to Landau damping can be observed in sheared flows of conventional fluids. ${ }^{18}$

While the Landau damping effect is kinetic in nature, some aspects of the phenomenon can be emulated within a fluid model using an idea originally due to G. W. Hammett and F. W. Perkins. ${ }^{16}$ Their basic idea was to develop a closure for the heat flux in terms of the temperature such that the decay rate predicted by linear Landau damping theory is reproduced by the fluid model when linearized. In one space dimension, the Fourier-space expression for this closure is given by the simple formula

$$
\hat{q}_{k}=-n_{0} \sqrt{\frac{8}{\pi}} v_{t h} i \operatorname{sgn}(k) \hat{T}_{k},
$$

where $n_{0}$ is the unperturbed plasma density, $v_{t h}$ is the nominal plasma thermal speed, $i$ is the imaginary unit, and sgn is the signum function. In a periodic domain the formula still makes sense provided the wave vector $k$ is suitably quantized.

Up to a proportionality factor the formula (10) says that the heat flux is related to the temperature by the Hilbert transform from signal analysis and elsewhere in plasma physics. ${ }^{19,20}$ In the signals context, the transform is used, for example, to construct complex representations of signals that are analytic in the upper half-plane, while in the plasma context the transform is required to find the action-angle variables for the continuous spectrum of the linearized Vlasov-Poisson system. If $H$ denotes the Hilbert transform, and we neglect the unimportant constants, the closure relationship may be written $q(x)=H[T](x)$. There are a number of well-known formulas for the Hilbert transform including

$$
H[T](x)=\frac{1}{\pi} P \int_{-\infty}^{\infty} \frac{T(s)}{x-s} d s,
$$

where $P$ denotes the principal value, and

$$
H[T](x)=-\frac{1}{\pi} \lim _{\varepsilon \rightarrow 0} \int_{\varepsilon}^{\infty} \frac{T(x+s)-T(x-s)}{s} d s,
$$

each of which explicitly displays the spatially-global nature of the closure. However, where the Fourier space expression (10) needs only minor modification in a periodic domain (a quantization condition on $k$ ) the previous two expressions require more significant changes. For instance the principal value formula (11) on a domain with period $2 \pi$ becomes

$$
H[T](x)=\frac{1}{2 \pi} P \int_{0}^{2 \pi} T(s) \cot \left(\frac{x-s}{2}\right) d s .
$$




\section{Collisionless limit: Guo-Tang closure}

In a long mean-free-path ( $\left.\lambda_{\mathrm{mfp}}\right)$ magnetized plasma, $C(f) \approx 0$, significant temperature anisotropy can develop. Further, if the plasma under consideration experiences an open magnetic field line, in contrast to the scenario for the Hammett and Perkins closure, ${ }^{16}$ it can be demonstrated that parallel heat flux can drive heat from low- to high- $T_{\|}$in the vicinity of absorbing boundary walls, contradicting the classical thermal conduction description of heat flux i.e. $q \sim \kappa \nabla T$. In this scenario, a closure derived by Guo and Tang ${ }^{17,21}$ has been demonstrated to sufficiently capture the behavior of the parallel heat flux in the presence of an absorbing boundary with open magnetic field line transport. In the Guo-Tang result, closure expressions for both the electron and ion parallel heat flux components are presented. In this study we solely examine the more physically-interesting electron result.

The Guo-Tang closure is applicable to electron transport within an open-field-line plasma bounded by absorbing walls. Steady-state is achieved via balance with an upstream plasma source with Maxwellian velocity distribution. Throughout the domain, electrons experience an open-line magnetic field, $\boldsymbol{B}$, and ambipolar electric potential, $\boldsymbol{\phi}$. A schematic example of this scenario is shown in Figure 1, indicating source flux, $\Gamma_{s}$, temperature $T_{s}$, wall potential $\phi_{w}$, as well as magnetic field magnitude at the source, $B_{S}$, and wall $B_{w}$.

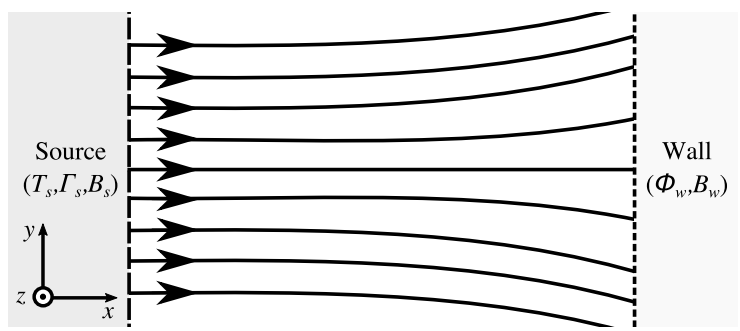

Figure 1. Geometry of Guo-Tang closure scenario ${ }^{17}$. A symmetric flux expander where the plasma is uniform in the $\mathrm{z}$ direction (out of plane) and symmetric about the source plane. Source parameters, $\Gamma_{s}$ and $T_{s}$, wall potential, $\phi_{w}$, and magnetic field magnitudes, $B_{s}$ and $B_{w}$, are employed in non-local closure.

This closure relates the parallel heat flux, $\boldsymbol{q}_{\|}$, to contributions from parallel, $q_{n}$, and perpendicular, $q_{s}$, thermal energies

$$
\boldsymbol{q}_{\|}=\left(q_{n}+2 q_{s}\right) \hat{\boldsymbol{b}}
$$


The parallel, $q_{n}$, and perpendicular, $q_{s}$, contributions to the total heat flux are given by

$$
\begin{aligned}
& q_{n}=\left\langle m_{e} v_{e \|}^{3}\right\rangle-3 n_{e} u_{e} T_{e \|}, \\
& q_{s}=\left\langle\mu_{e} B v_{e \|}\right\rangle-n_{e} u_{e} T_{e \perp},
\end{aligned}
$$

where the $n_{e} u_{e} T_{e}$ components represent convective fluxes available from the moment variables used in computation of the fluid model. The crux of the heat flux closure is in the evaluation of both $\left\langle m_{e} v_{e \|}^{3}\right\rangle$ and $\left\langle\mu_{e} B v_{e \|}\right\rangle$, given by

$$
\begin{gathered}
\frac{1}{2}\left\langle m_{e} v_{e \|}^{3}\right\rangle=\frac{\Gamma_{s} T_{s}}{2 R^{2}}\left[D+R\left(\frac{3}{2}-\frac{1}{R}+\frac{e \phi}{T_{s}}\right) \operatorname{erfc}\left(\sqrt{\tilde{\phi}_{w}}\right)\right. \\
\left.+C\left(R \alpha \frac{e \phi}{T_{s}}+R G-G-\frac{1}{2}\right) \operatorname{erfi}\left(\sqrt{\alpha \tilde{\phi}_{w}}\right)\right], \\
\left\langle\mu_{e} B v_{e \|}\right\rangle=\frac{\Gamma_{s} T_{s}}{2 R^{2}}\left[-D+\operatorname{erfc}\left(\sqrt{\tilde{\phi}_{w}}\right)+C\left(G+\frac{1}{2}\right) \operatorname{erfi}\left(\sqrt{\alpha \tilde{\phi}_{w}}\right)\right],
\end{gathered}
$$

where $R=B_{s} / B, e$ is the elementary charge, $\operatorname{erfc}()$ and erfi() are the complementary and imaginary error functions, and constants are defined as $\alpha=B_{w} /\left(B_{s}-B_{w}\right), \tilde{\phi}_{w}=e\left|\phi_{w}\right| / T_{s}$, $C=\mathrm{e}^{-(1+\alpha) \tilde{\phi}_{w}} / \alpha^{3 / 2}, D=\mathrm{e}^{-\tilde{\phi}_{w}} \sqrt{\tilde{\phi}_{w} / \pi}(1+\alpha) / \alpha, G=\alpha\left[3 / 2+(1+\alpha) \tilde{\phi}_{w}\right]$.

In equations (17) and (18) the non-local dependence of the source and wall parameters on local quantities is explicit via $\Gamma_{s}, T_{s}, \alpha$, and most prominently $\phi_{w}$, which influences multiple terms. The explicit non-local dependence in equations (17) and (18) echoes the observation of Hazeltine, ${ }^{6}$ in that the influence of the source and wall should enter explicitly in establishing a clear non-local description of the parallel heat flux. Due to its accounting for crucial non-local physics via source and boundary effects, this closure provides a useful benchmark limit for our study.

\section{DETAILS OF THE LEARNING METHOD}

In this section, we outline a workflow for machine learning the three types of closure introduced previously. The focus will be on replicating results of analytical expressions using neural network formulations. Individual networks for each closure will be designed according to the physical assumptions utilized in their analytical counterpart. In this way, we aim to provide a framework for generating closures in a physics-informed manner that simplifies the network designer's task for synthesizing closure surrogates. We note that the ultimate goal of this line of research is to generate closures that can deploy differing underlying assumptions according to localized deployment requirements. 


\section{A. A surrogate Braginskii closure}

The underlying assumptions of the Braginskii closure rely on the specification of an analytical expression which is pointwise in nature. To that end, our problem is formulated in a supervised learning framework wherein a deep neural network is tasked with predicting the Braginskii closure at discrete locations on a grid, locally. Labels for the training data are generated using the analytical form of the closure and framed in a feature-target representation where the features (or inputs) are given by the density $n(\boldsymbol{x})$, velocity $\boldsymbol{u}(\boldsymbol{x})$, gradients of velocity $\nabla_{\boldsymbol{x}} \boldsymbol{u}(\boldsymbol{x})$, and temperature $T(\boldsymbol{x})$ at a given point.

Training and testing data for the Braginskii closure surrogate was generated via equation (8) from randomly generated profiles for $(n, \boldsymbol{u}, T)$ under a uniform magnetic field given by $\boldsymbol{B}=$ $\boldsymbol{e}_{x}+0.5 \boldsymbol{e}_{y}+2 \boldsymbol{e}_{z}$ in Cartesian coordinates. These profiles were specified to be continuous, smooth, and physical (i.e. $n, T>0$ ) via random mode sinusoids

$$
\begin{gathered}
n(x, y, z)=0.5 \sin \left(\alpha_{1} x\right) \sin \left(\alpha_{2} y\right) \sin \left(\alpha_{3} z\right)+0.5, \\
u_{x}(x, y, z)=\sin \left(\alpha_{4} x\right), \\
u_{y}(x, y, z)=\cos \left(\alpha_{5} y\right), \\
u_{z}(x, y, z)=\sin \left(\alpha_{6} z\right), \\
T(x, y, z)=0.5 \cos \left(\alpha_{7} x\right) \sin \left(\alpha_{8} y\right) \cos \left(\alpha_{9} z\right)+0.5,
\end{gathered}
$$

where random modes $0 \leq \alpha_{i} \leq 3$ are generated from a uniform distribution. The target variables are given by the upper-diagonal components of $\Pi^{\mathrm{BG}}(n, \boldsymbol{u}, T)(\boldsymbol{x})$. Note that for the choice of training profiles the diagonal components are all zero and the target tensor is symmetric.

\section{B. A surrogate Hammett-Perkins closure}

In contrast to the Bragisnkii closure, the Hammett-Perkins closure captures nonlocal physics (associated with Landau damping) in a collisionless setting. This nonlocality may be inferred from the analytical expression, Eq. (10), for the Hammett-Perkins closure function written in Fourier space. Our learning task was to train a neural network to map inputs given as $N$-component vectors $\mathbf{T}$ of nodal temperature values into $N$-component vectors $\mathbf{Q}$ of nodal heat flux values. For training data we drew random temperature vectors $\mathbf{T}$ in $\mathbb{R}^{N}$ from a multi-variate normal distribution with 
mean $\overline{\mathbf{T}}$ and covariance $\mathbf{C}$ given by

$$
\begin{aligned}
\bar{T}_{i} & =1 \\
C_{i j} & =\sigma^{2} \exp \left(\frac{-2 \sin \left(\pi\left(x_{i}-x_{j}\right) / T_{p}\right)^{2}}{L_{c}^{2}}\right) .
\end{aligned}
$$

Here $T_{p}$ and $\sigma^{2}$ are positive constants, the $x_{i}$ are uniformly-spaced nodes on the 1-dimensional spatial domain $\left[0, T_{p}\right]$, and $L_{c}$ is a parameter that sets the correlation length of the samples. The form of the covariance (25) ensures that samples are periodic in the sense that $T_{1}=T_{N}$.

This method of data generation corresponds to assuming temperature profiles the closure function will see are independent realizations of a Gaussian random field with constant mean temperature and covariance $\left\langle\tilde{T}(x) \tilde{T}\left(x^{\prime}\right)\right\rangle=\sigma^{2} \exp \left(\frac{-2 \sin \left(\pi\left(x-x^{\prime}\right) / T_{p}\right)^{2}}{L_{c}^{2}}\right)$. We adopt this assumption for demonstration purposes only. In real-world applications of neural-network-based closures the distribution that the training data is drawn from must be consistent with problem-specific physics. Like all training data generated in this study, the normal distribution specified by Eqs. (24)-(25) plays the role of a generic placeholder for a physical distribution of temperature profiles.

A similar learning exercise was also carried out recently using a different training data distribution by Ma et al.. ${ }^{15}$ Like this earlier reference, we will focus on learning the closure in configuration space in order to mask its trivial nature in Fourier space. In contrast to the earlier study, our analysis will focus on the important issue of extrapolation errors inherent to artificial neural networks. We frame this extrapolation problem concretely as follows. If a neural network surrogate model for the Hammett-Perkins closure is trained using data sampled with mean $\bar{T}_{1}(x)$ and covariance $\tilde{T}_{1}\left(x, x^{\prime}\right)$ then how well does the learned closure perform on testing data sampled with mean $\bar{T}_{2}(x)$ and covariance $\tilde{T}_{2}\left(x, x^{\prime}\right)$ ? In any future application of neural networks to the problem of closure learning for plasma physics the limitations of the learning process set by extrapolation errors will be crucial to understand and manage. This will be demonstrated through visualization and quantitative assessments in the following sections.

\section{A surrogate Guo-Tang closure}

The collisionless Guo-Tang closure, similar to the Hammett-Perkins closure, requires some utilization of non-local context. Training and testing data for the Guo-Tang closure is generated by evaluating non-local closure variables in equations (17) and (18) for a variety of randomly generated inputs of magnetic field, $B$, and ambipolar potential, $\phi$, profiles. Physical constraints 
require these profiles to be monotonically decreasing. ${ }^{17}$ To generate a sufficiently-rich family of monotone training profiles, we use the exponential of a Gaussian cumulative distribution function, with random mean and variance. Over a domain $0 \leq x \leq 100$ we generate input profiles

$$
\begin{gathered}
B(x)=B_{S} \exp \left(-\frac{1}{2}\left[1+\operatorname{erf}\left(\frac{x-\hat{X}_{1}}{\sqrt{2} \sigma_{1}}\right)\right]\right), \\
\phi(x)=\exp \left(-\frac{1}{2}\left[1+\operatorname{erf}\left(\frac{x-\hat{X}_{2}}{\sqrt{2} \sigma_{2}}\right)\right]\right),
\end{gathered}
$$

where erf is the error function, the parameters $1 \leq B_{s} \leq 2$, Gaussian means $10 \leq \hat{X}_{i} \leq 90$ and variances $10 \leq \sigma_{i}^{2} \leq 60$ are randomly generated from a uniform distribution. Further, the values for temperature, $0.5 \leq T_{s} \leq 2$, and flux of source electrons, $1 \leq \Gamma_{s} \leq 3$, used to evaluate equations (17) and (18) are also randomly generated from a uniform distribution.

With a repeatable prescription for generating training data defined, we will seek to develop suitable network architectures that mimic the spatially non-local relationship between the local inputs, $B(x)$ and $\phi(x)$, the boundary quantites, $\Gamma_{s}, T_{s}, B_{w}, B_{s}$, and $\phi_{w}$, and the spatially varying heat flux component outputs, $\left\langle m_{e} v_{e \|}^{3}\right\rangle(x)$ and $\left\langle\mu_{e} B v_{e \|}\right\rangle(x)$.

\section{MACHINE LEARNING ARCHITECTURES}

For the purpose of building surrogates for each closure hypothesis, we test three different types of machine learning frameworks given by fully connected, convolutional, and locally connected artificial neural networks. A brief introduction is provided to all three frameworks and we demonstrate that each framework is associated with certain advantages (as well as disadvantages). Consequently, we outline the need for surrogate closure development from data based on the underlying hypotheses in a physics-informed manner. In addition, we also comment on the choice of optimal hyperparameters for each of these frameworks and how these may be tied to the nature of nonlocality in closure requirements. The various network architecture introduced in this section are described schematically in Figure 2.

\section{A. Fully connected neural network}

A fully connected neural network (FNN) consists of a series of global operations that nonlinearly transform a set of inputs to a set of outputs given by input and output training data. An 
FNN achieves this by performing multiple matrix operations on an input vector to map to an output vector given by the dimensionality of the targets (i.e., quantities to be predicted). The multiple matrix operations may or may not be used for transforming the size of the input vector as it "flows" through the network. Each matrix operation is followed by potential nonlinear transformations of the transformed vector by activations which are element-wise operations of nonlinear functions such as the sigmoid, hyperbolic tangent or rectified linear functions. An example of a sigmoidal activation is

$$
\varphi(\beta)=\left(1+e^{-\beta}\right)^{-1}
$$

where $\beta$ is a component of a vector. Similarly the hyperbolic tangent (also known as tanh) activation is given by

$$
\varphi(\beta)=\left(e^{\beta}-e^{-\beta}\right) \cdot\left(e^{\beta}+e^{-\beta}\right)^{-1}
$$

and the rectified linear activation (also denote ReLU) is given by

$$
\varphi(\beta)=\max [0, \beta]
$$

We note that ReLU is commonly used for its success in very deep networks as it prevents the saturation inherent in negative exponentiation in the sigmoid and tanh activations. The general operation for one layer of an FNN may be expressed as

$$
q_{i}^{(l)}=\varphi\left(\sum_{j} W_{i j}^{(l)} q_{j}^{(l-1)}\right)
$$

where an input signal $\left.q_{j}^{(} l-1\right)$ from layer $l-1$ is transformed by a matrix multiplication with $W_{i j}^{l}$ (which can be optimized for) and a nonlinear activation by $\varphi$. In this article, we have utilized the ReLU activation function for all hidden layers except the output layer which is kept as a linear operation.

\section{B. Convolutional neural network}

Convolutional neural networks (CNNs) are a class of deep, feed-forward artificial neural networks that are commonly applied to analyzing images. CNNs assume that the underlying images (and in our case field quantities) are stationary (i.e., statistics of one part of the image are the same 
as other) and that a spatially local correlation exists between the image pixels. The convolutional layer is the core building block of a CNN and consists of filters whose size defines the extent of spatial locality assumed; each filter corresponds to a specific feature or pattern in the image. The convolution operation using these filters in each layer ensures stationarity and thus translational invariance. Several convolutional layers are stacked in such a way that the complex image features are learned hierarchically by composing together the features in previous layers and a general operation within one layer of a $\mathrm{CNN}$ is given as follows:

$$
q_{i j m}^{(l)}=\varphi\left(\sum_{k=0}^{K-1} \sum_{p=0}^{L-1} \sum_{s=0}^{L-1} h_{p s k m}^{(l)} q_{i+p, j+s, k}^{(l-1)}\right)
$$

where $K$ is the number of field variables available on the grid, $h$ refers to the trainable filter, $L$ are the degrees of freedom in each dimension of an image (assumed to be two-dimensional in this equation) and $\varphi$ is our previously introduced choice for activation. Note that the number of grid variables in the transformed signal can be changed from $K$ depending on the the number of filters $h$ corresponding to different $m$. The CNN architectures are ideal for analyzing images, are more efficient to implement than fully connected models, and vastly reduce the number of parameters in the network, thus decreasing the memory requirement and training time. However, it is generally observed that a greater amount of training data is needed for learning, particularly for spatial maps that need to be deployed pointwise. Greater details may be found in the seminal work of Ref. 22, which led to mainstream utilization of CNNs for image type problems.

\section{Locally connected neural network}

A locally connected neural network (LNN) is a hybrid between CNN and FNN architectures. It leverages non-local relationships in its input layer through feature engineering before using a fully connected network to map to the target values. More importantly, in the process of feature engineering, different variables at each grid point in the field are collected to obtain a sample. In this way, relationships are learned point-wise without passing entire fields through the framework of large network architectures. Far simpler neural networks are obtained and training can be performed with fewer generation of fields since the number of samples is dramatically increased. The reader is directed to an example of such networks in ${ }^{23}$ for grid based learning problems. Note that for physics which are inherently global, this approach is limited as shall be demonstrated for the Hammett-Perkins closure. 

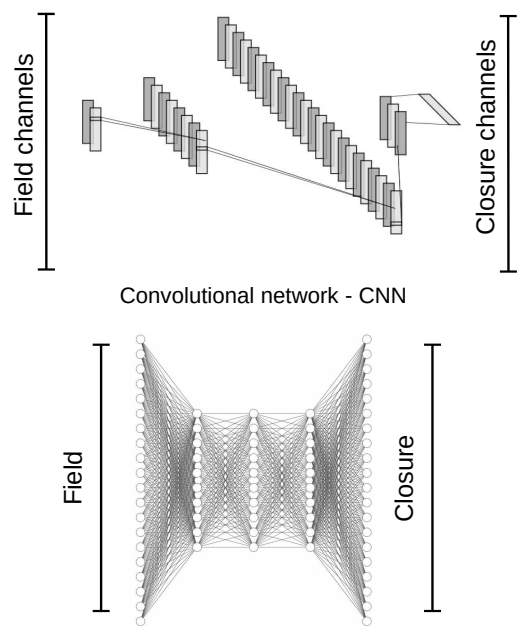

Fully connected network -FNN

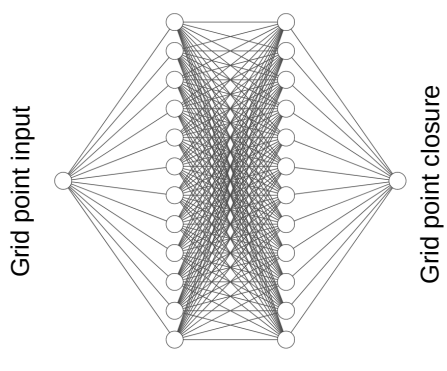

Locally connected network - LNN

Figure 2. Schematics for the three different network architecture considered in this study. The first architecture (top) refers to the convolutional neural network (CNN) which takes the entire field as an input but uses local kernels to learn translation invariant structures in the input fields, the second architecture is the fully connected neural network (FNN) that also takes the entire field as an input. The bottom schematic shows a simple locally connected neural network (LNN) which is agnostic to the discretization or query location in the field and solely makes point-wise predictions. 


\section{RESULTS}

In this section we utilize statistical assessments for diagnosing the accuracy of machine-learned predictions for the different types of architectures. These assessments are made through plots showing the probability distribution of machine learning responses compared to those of the truth, generated by the analytic closures. We also show scatter plots comparing predictions and truth. Each type of closure is assessed with the three types of neural network formulations we have outlined in the previous section. Networks are assessed in terms of accuracy of predictions, the number of training parameters, and the cost of training. We then make conclusions about architecture selection given a priori understanding of the non-locality of a closure modeling scenario. All our assessments were performed using TensorFlow v1.15 $5^{24}$ on a single-node Intel Core-i $78^{\text {th }}$ generation processor, with a clock speed of approximately $1.90 \mathrm{GHz}$ on a Ubuntu 18.04 operating system. GPU computing was not used in this study.

All the networks assessed in the experiments of this section utilize a learning rate of 0.001 . The learning rate is a tuning parameter in an optimization algorithm that determines the step size at each iteration while moving toward a minimum of the loss function. For all exercises in this study the standard Adam optimizer was used. ${ }^{25}$ With regards to the early stopping criteria, a section of the training data would be held out and not used for optimization (i.e., for validation) and training would be terminated if errors on this set were observed to be higher than the previous best error for more than 10 epochs. Batch sizes were varied for optimal throughput for all the experiments, although results were observed to be relatively robust to different choices.

For each of the three closure training exercises, all network architecture styles were fed the same quantity of input data. While the current training data is trivially generated from analytic expressions, it acts only as a placeholder for more valuable high fidelity training data to be used in future studies. Our reasoning behind this constraint is that we consider computationally expensive high fidelity training data, for example from a 6D kinetic equation or particle method, to be a premium commodity and therefore we value the ability of a network architecture to learn a reasonable surrogate without an onerous need for training data. 


\section{A. Learning Braginskii}

We outline the results from using the machine learning model for predicting the Braginskii gyroviscous stress tensor in this subsection. For training and validation of closures for this framework, the input quantities were sampled from 10 fields of nodal values of $(\mathbf{u}(\mathbf{x}), \nabla \mathbf{u}(\mathbf{x}))$ on a $N_{x} \times N_{y} \times N_{z}=50 \times 50 \times 50$ mesh. Ground-truth values, i.e. labels for the data, were generated by applying the analytic expression for the gyroviscous stress tensor to the sampled inputs. We note that the predictions were for the off-diagonal components of a symmetric tensor with zeros along the diagonal (i.e., there were three only outputs from each query of the network). We shall provide three sets of results for the testing data, which is sampled from a completely different randomly generated field of inputs and outputs. Therefore, these plots describe the learning prowess of the networks on the data that it has not seen during trainable parameter optimization.

We first outline results from the LNN framework. Here, training and validation utilized 6000 data points (5400 for training and 600 for validation). These were obtained randomly from the ten fields mentioned previously to allow for faster learning of the closure framework. We observed that the accuracy of the closure was not affected by this choice. This network architecture utilized each data point on the grid as a training sample and therefore built a map from 14 inputs (corresponding to the density, velocity, velocity gradient, and temperature information available from the grid) to 3 outputs corresponding to the closure terms. A grid-based hyperparameter search was performed to obtain the most accurate framework based on the lowest validation loss. Our grid consisted of a sweep of two-dimensions: given by the number of neurons in a hidden layer and the number of hidden layers. While more dimensions may have been included in this scan, the two-dimensional configuration was a result of compute cost considerations. Our networks were obtained from a choice of 10, 30, 50, 70 hidden layer neurons and 1, 3, 5 or 7 hidden layers with a fixed batch size of 128 . The best network was given by 3 layers and 50 neurons based on validation loss. This trained network was then tested on a held-out data set as shown in the following. Figure 3 shows the probability density functions (PDFs) for the true and predicted values of the testing data set when using the best LNN obtained after a hyperparameter search. Response trends for each component are reproduced appropriately by this version of the surrogate formulation. Figure 4 shows the corresponding scatter plots for the same predictions. The 45 degree line represents the true values plotted against themselves. The predictions are seen to be quite close to the true values, represented by the predicted scatter points being close to the true line. We note that the 
coefficient of determination of this experiment was around $R^{2}=0.993$ indicating a very good fit. Note that the $R^{2}$ values for the Braginskii experiment are obtained by averaging those for each component of the predicted tensor. Finally we include two-dimensional scatter plots that characterize the relationship between the different components of the predicted stress tensor in Figure 5 for predictions from our LNN framework. One can observe that the true relationship between the different components is recovered by the predicted ones quite accurately.

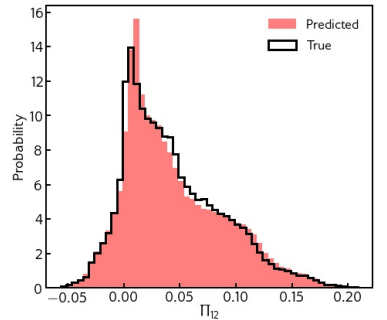

(a)

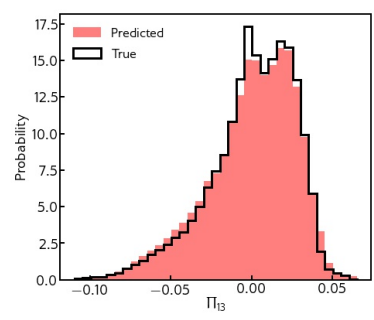

(b)

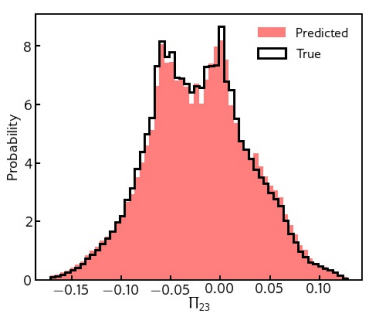

(c)

Figure 3. Probability density functions of upper diagonal Braginskii stress tensor elements (a) $\Pi_{12}$, (b) $\Pi_{13}$, (c) $\Pi_{23}$ showing true and values predicted by the trained locally connected machine learning framework. Note that this data is a part of testing set showing the generalizability of the trained network.

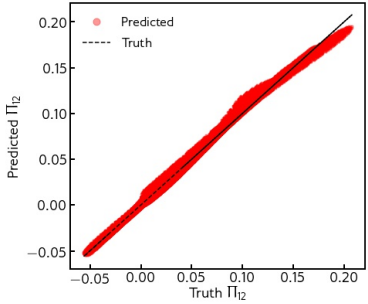

(a)

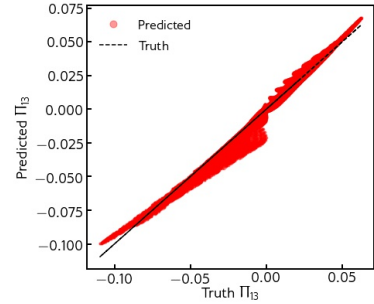

(b)

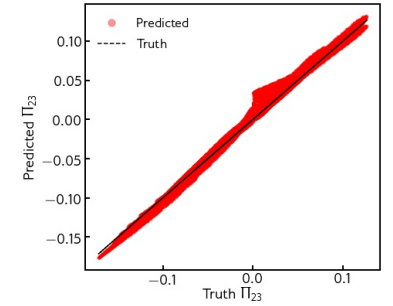

(c)

Figure 4. Scatter plots of upper diagonal Braginskii stress tensor elements (a) $\Pi_{12}$, (b) $\Pi_{13}$, (c) $\Pi_{23}$ describing the accuracy of the locally connected machine learning framework. Deviations from the 45 degree line indicate erroneous predictions. Note that this data is a part of testing set showing the generalizability of the trained network.

Next we assess the ability of a CNN for the same prediction task. For this experiment, we utilize all 10 data fields for training and validation. We note that this is motivated by recognizing 


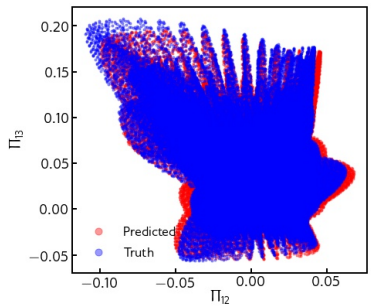

(a) $\Pi_{12} \vee \Pi_{13}$

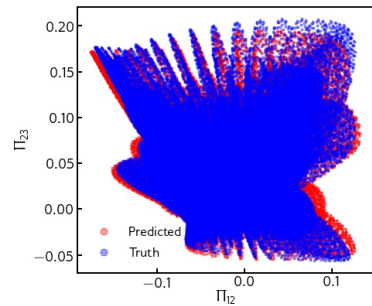

(b) $\Pi_{12} \vee \Pi_{23}$

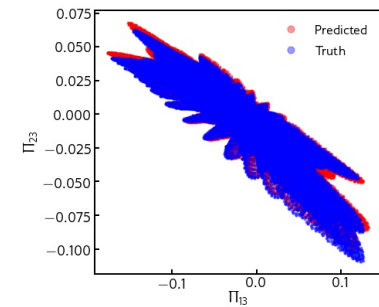

(c) $\Pi_{13} \vee \Pi_{23}$

Figure 5. Two-dimensional contour plots showing the relationships between different components of the closure terms for both true and predicted stresses. The spread of the data points in the two-dimensional space is recreated accurately by the LNN architecture.

that each data field is one data point for the CNN. For training we utilize 9 while keeping 1 aside for validation and early stopping. Thus, this framework is trained on 10 times more points than the LNN framework. An entire set of grid variables are interpreted as inputs and the closure terms on all grid points are our outputs. Like in the previous experiment with the LNN framework, we perform a two-dimensional grid-based hyperparameter scan to select the best architecture by lowest validation loss. Our options are given by different kernel sizes (i.e., the width of the filters within a $\mathrm{CNN}$ ) as well as different depths of the CNN. Our choices for kernel sizes are 2, 3, 4, 5 and we also select from CNNs with 3, 4, 5, 6 hidden layers. Note that the filter sequence for each hidden layer choice is given as follows: for 3 hidden layers we use $[30,20,10]$ as our number of filters, for 4 hidden layers we use $[30,20,15,10]$ as our filter configuration, for 5 hidden layers we use $[30,25,20,15,10]$ as our filters and for 6 hidden layers we use $[30,25,20,15,10,5]$ as our filter values. All architectures are trained with a batch size of 2 . The best architecture obtained through this sweep is one that has a kernel size of 3 and 6 hidden layers. Note that the filter sequence controls the dimensionality of the number of channels of the data as it is being transformed through the network. To elaborate, the input data has 14 channels corresponding to all the input quantities available on the grid and the output tensor contains 3 channels that correspond to the closure terms. No pooling layers are used in this study and zero padding is used to preserve the dimension of the field during the forward pass through the framework. The results for learning the Braginskii closure using the best found CNN are shown in terms of PDFs in Figure 6 and scatter plots in Figure 7, which show that the CNN performs poorly relative to the 
$\mathrm{LNN}$, even though the $\mathrm{CNN}$ training set had a richer information content. Our hypothesis is that a greater amount of training data may improve the learning of the $\mathrm{CNN}$ framework. We remind the reader that we have fixed, (a-priori), the total amount of training, validation and testing data for all experiments in this particular study for the purpose of a consistent comparison. However, we would like to remark that when data generation is costly the LNN method may prove a valuable addition to the workflow. The problem of generating adequate data for the best model performance cannot be answered until one carries out statistical assessments similar to the ones undertaken in this study. Indeed, an element of feedback (i.e., generate data, train models, assess on held-out dataset, repeat) is generally inevitable for most practical applications. The $R^{2}$ of this particular experiment was slightly lower, but still relatively accurate, at 0.87 . Finally we include the twodimensional scatter plots for the $\mathrm{CNN}$ predictions and compare them to the true data set as shown in Figure 8 which shows poorer agreement when compared to LNN, due to the reduced overlap between the true and learned relationships.

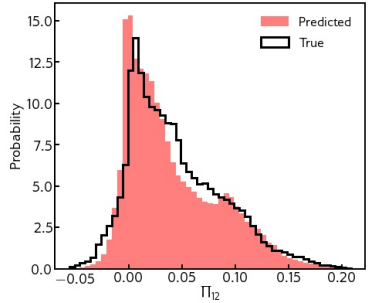

(a)

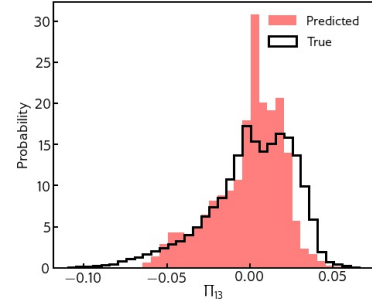

(b)

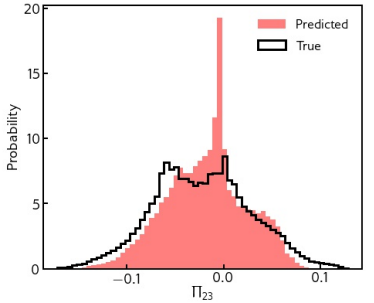

(c)

Figure 6. Probability density functions of upper diagonal Braginskii stress tensor elements (a) $\Pi_{12}$, (b) $\Pi_{13}$, (c) $\Pi_{23}$ showing true and values predicted by the trained $\mathrm{CNN}$ learning framework. Note that this data is a part of testing set showing the generalizability of the trained network.

Finally we show results from an FNN which interprets every field quantity at every point as a one dimensional vector and maps to the target tensor components also interpreted to be part of a large vector. Similar to the CNN experiment, we utilize all 10 fields for training and validation. For training we utilize 9 while keeping 1 aside for validation and early stopping. Thus, the FNN was also trained on 10 times more points than the LNN framework. Mathematically, our fully connected map is given by a nonlinear transformation from a space of dimension $N_{x} \times N_{y} \times N_{z} \times 14$ to $N_{x} \times N_{y} \times N_{z} \times 3$. One can note immediately that the use of an FNN that connects every point 


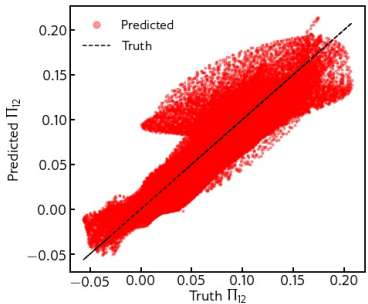

(a)

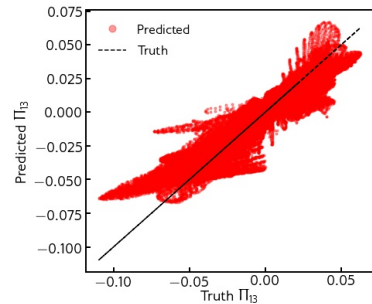

(b)

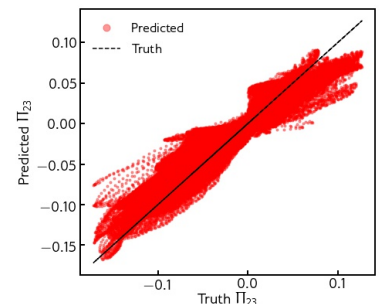

(c)

Figure 7. Scatter plots of upper diagonal Braginskii stress tensor elements (a) $\Pi_{12}$, (b) $\Pi_{13}$, (c) $\Pi_{23}$ describing the accuracy of the CNN framework. Deviations from the 45 degree line indicate erroneous predictions. Note that this data is a part of testing set showing the generalizability of the trained network.

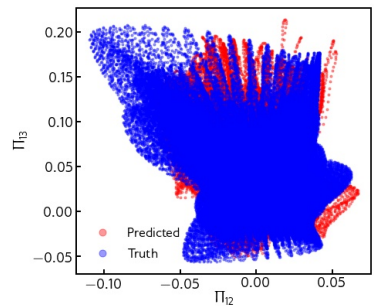

(a) $\Pi_{12} \vee \Pi_{13}$

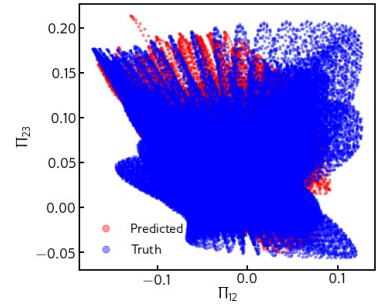

(b) $\Pi_{12} \vee \Pi_{23}$

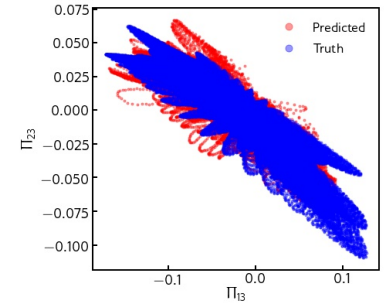

(c) $\Pi_{13} \vee \Pi_{23}$

Figure 8. Two-dimensional contour plots showing the relationships between different components of the closure terms for both true and predicted stresses. The spread of the data points in the two-dimensional space is recreated accurately by the $\mathrm{CNN}$ architecture but with less fidelity than the LNN architecture.

in the field is computationally infeasible. However, for the purpose of a thorough comparison, we perform a hyperparameter search specified for the previous frameworks. In particular, we use the same grid-based search as employed for the LNN framework - i.e., options of 10, 30, 50 and 70 hidden layer neurons combined with 1,3,5, and 7 hidden layers. All architectures were trained with a batch size of 2 . Our best network by validation loss is then found to be a five-layered network with 10 neurons. Results from this network are shown in Figures 9 (for the PDF) and Figures 10 for the true-predicted scatter plots which indicate far poorer performance than the CNN and the LNN frameworks. Figure 11 also shows the quality of the two-dimensional scatter plots capturing the correlation between the true and predicted quantities where the FNN framework is 
seen to perform poorly. Finally, $R^{2}$ values of approximately -0.97 indicate the poor performance of this framework.

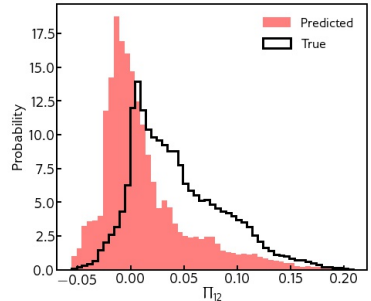

(a)

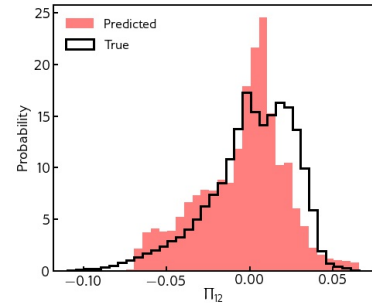

(b)

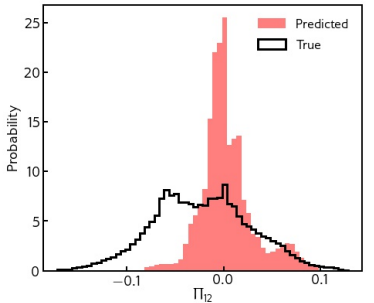

(c)

Figure 9. Probability density functions of upper diagonal Braginskii stress tensor elements (a) $\Pi_{12}$, (b) $\Pi_{13}$, (c) $\Pi_{23}$ showing true and values predicted by the trained fully connected neural network learning framework. Note that this data is a part of testing set showing the generalizability of the trained network.

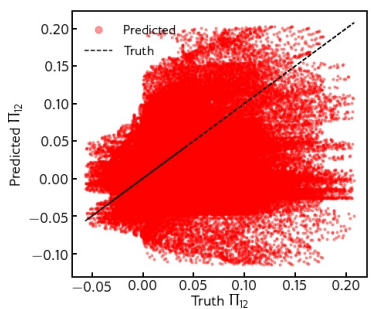

(a)

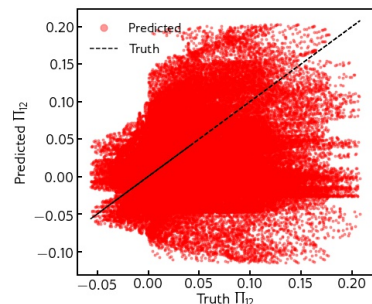

(b)

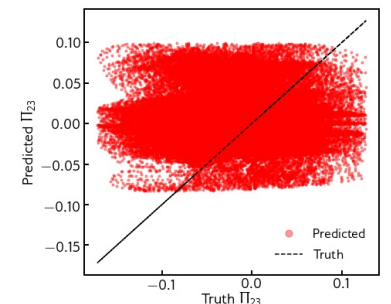

(c)

Figure 10. Scatter plots of upper diagonal Braginskii stress tensor elements (a) $\Pi_{12}$, (b) $\Pi_{13}$, (c) $\Pi_{23}$ describing the accuracy of the FNN framework. Deviations from the 45 degree line indicate erroneous predictions. Note that this data is a part of testing set showing the generalizability of the trained network.

To ensure that the conclusions about the appropriate networks for the Braginskii closure are not due to data or architectural artifact, we detail the different validation losses from the hyperparameter search for each of the LNN, CNN and FNN framework in Figure 12 through the use of box-plots. A box-plot for each architecture provides the following information: the line in the middle of the box informs the reader of the median of the different validation losses, the bottom and top edges of the box indicate the $25^{\text {th }}$ and $75^{\text {th }}$ percentile of architectures ordered by increasing validation losses, the bottom and top whiskers indicate the $5^{\text {th }}$ and $95^{\text {th }}$ percentile of the same 


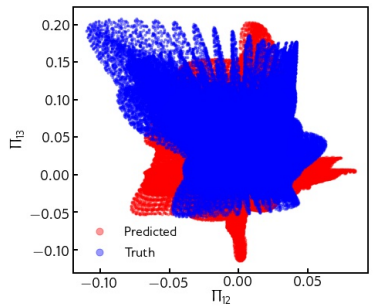

(a) $\Pi_{12} \vee \Pi_{13}$

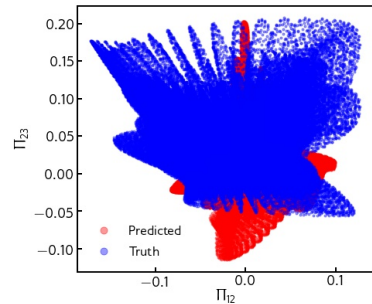

(b) $\Pi_{12} \vee \Pi_{23}$

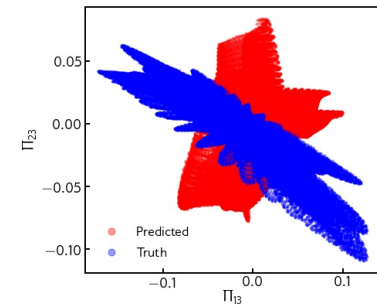

(c) $\Pi_{13} \vee \Pi_{23}$

Figure 11. Two-dimensional contour plots showing the relationships between different components of the closure terms for both true and predicted stresses. The spread of the data points in the two-dimensional space is recreated poorly by the FNN architecture given the current size of the training data set.

and values beyond the whiskers are denoted outliers. It is apparent that the LNN architecture, even when varying the hyperparameters, obtains better architectures by validation loss than the $\mathrm{CNN}$ and FNN variants with the lowest median loss of around $2 \times 10^{-6}$. The CNN framework is also able to obtain better performance than the FNN architectures with a median value of approximately $2 \times 10^{-4}$. The FNN performs uniformly poorly across the different architectures as shown with all validation losses being approximately equal.

\section{B. Learning Guo-Tang}

We proceed by assessing the ability of the machine learning frameworks for predicting the heat flux in the Guo-Tang framework. Our results are from two experiments: one with input information from solely local quantities, and a second experiment that introduces boundary and source information to every point of the physical domain to allow for a physics-informed use of machine learning. We generate data on a uniform grid with 256 points for our inputs and outputs. For the LNN framework, each location on the grid constitutes an independent data point whereas for FNN and CNN frameworks, each realization of inputs and outputs on the entire grid represents one data point. To accommodate the latter, we generate data on this grid 10,000 times. Of these 10,000 fields, 6300 are utilized for the purpose of training, 700 are utilized for validation and 3000 are retained for testing. In contrast to the previous three-dimensional experiment, a 'field' now refers to a one-dimensional instance of multiple input and output variables defined on the entire 


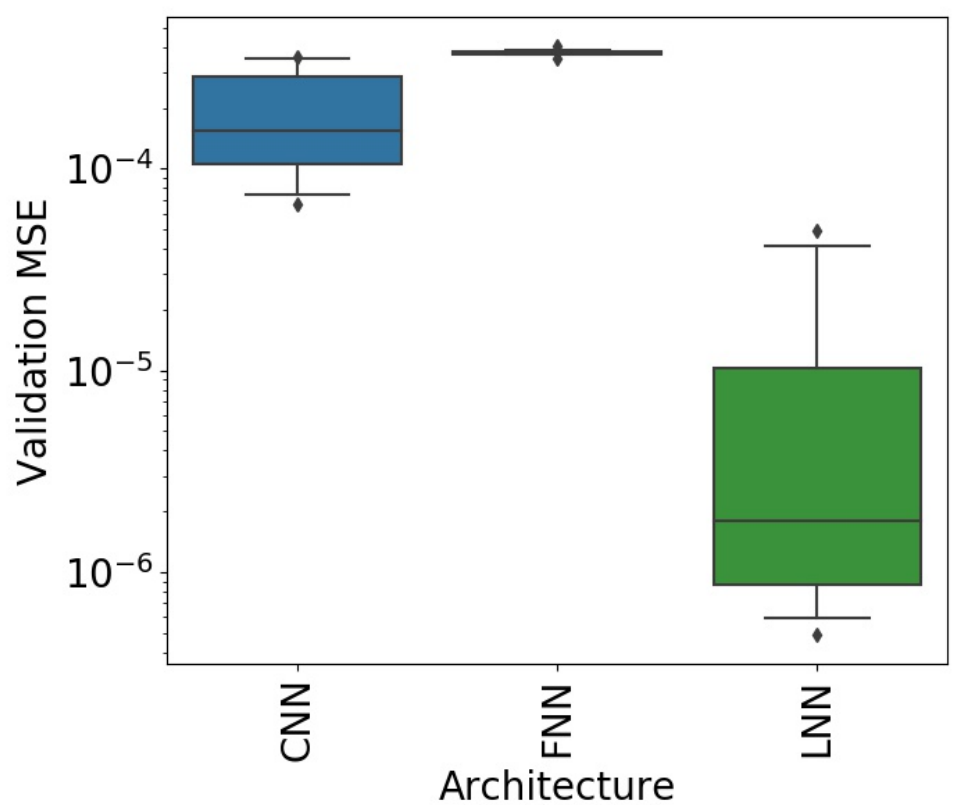

Figure 12. A grid-based hyperparameter search comparing accuracies for the LNN, CNN and FNN architectures trained for the Braginskii test case. The box plots are shown for validation mean-squared errors. The whiskers indicate the $5^{\text {th }}$ and $95^{\text {th }}$ percentile of evaluated models. The edges of the box indicate the $25^{\text {th }}$ and $75^{\text {th }}$ percentiles and the line within a box indicates the median value. It is apparent that the LNN performs better than the other architectures for this closure.

grid.

We first outline results for training on inputs given by local information alone. Our first framework for assessment is the LNN framework used to construct the local map between grid variables given by an input space of dimension 2, given by the magnetic field and ambipolar potential, to the outputs, given by parallel and perpendicular fluxes. Like for the previous assessments with the Braginskii learning, we perform a hyperparameter search to find the best number of layers and neurons for the framework. We note that the choices for layers and neurons are kept the same as the LNN hyperparameter search in the previous section. Figure 13 shows results from the optimal architecture found by a hyperparameter search with 7 layers and 10 neurons while using a batch-size of 1024. The absence of boundary information leads to poor performance - a theme 
that repeats itself across different types of neural network frameworks. Testing $R^{2}$ values of 0.349 were obtained for this particular experiment. Note that the $R^{2}$ values for the Guo-Tang experiment are obtained by averaging those for each component of the predicted output.

The results from the best $\mathrm{CNN}$ architecture obtained after a hyperparameter search are shown in Figure 14. Our hyperparameter search obtained architectures from the same possible choices as outlined in the Braginskii search in the previous section. The optimal architecture was given by a 5 layer network with a filter sequence given by $[30,25,20,15,10]$ and a filter kernel size of 5 . This training also utilized a batch size of 1024. Not much difference is observed between the LNN and the $\mathrm{CNN}$ framework for this particular configuration indicating that the lack of any boundary information causes low quality performance across neural architectures. Testing $R^{2}$ values of 0.059 are obtained for this deployment.

The results from the best FNN architecture obtained after a hyperparameter search are shown in Figure 15. As for the LNN and CNN frameworks, the hyperparameter options were fixed from the previous experiment. The best architecture was given by a 7 layer network with 70 neurons in each hidden layer to construct the local map between grid variables given by an input space of dimension $256 \times 3$ and output space of dimension $256 \times 2$. This training also utilized a batch size of 1024. An important point to note here is that the FNN architecture performs better than the CNN and LNN frameworks described previously with a testing $R^{2}$ of 0.483 . When compared with the hyperparameter search box plots shown in Figure 16, this fact is surprising since the LNN framework is seen to perform the best by measure of validation mean-squared errors. We attribute this to the fact that the best LNN framework overfits on the training data and thus achieves poorer performance on this testing data set. This is also shown in Figure 17 where the two-dimensional scatter of the FNN is superior to the LNN and CNN. In any case, as shown in the plots, the machine learned models prove poor almost universally. In the next experiment, we address this issue by explicitly introducing boundary information within the input variables used to train the networks.

We now show results from learning assessments where the input information is augmented with boundary quantities. In particular, each point of the internal domain is explicitly given boundary information related to magnetic field, ambipolar potential, source electron temperature and flux, $B_{w}, B_{s}, \phi_{w}, T_{s}$, and $\Gamma_{s}$ in addition to the local quantities, $B$ and $\phi$ at each grid point. As demonstrated below, we observed remarkable improvements in accuracy due to this data preprocessing step.

Figure 18 shows the PDFs of the true and predicted values of the parallel and perpendicular 
flux for a 5 layer 70 neuron LNN obtained after our standard hyperparameter search. In addition we also show scatter plots and a sample realization of the true and predicted closure profile for one prediction. The LNN is able to obtain the right trends in the parallel and perpendicular fluxes from the point of view of PDFs and the scatter plots show a good agreement as well. The LNN constructs a local map between grid variables now given by an input space of dimension 7 , corresponding to the magnetic field, ambipolar potential, source electron temperature and flux, $B_{w}, B_{s}, \phi_{w}, T_{s}$, and $\Gamma_{s}$ and output space of dimension 2, given by the two parallel and perpendicular components of the heat flux closure. We note that our grid is of dimension 256 but data at each location of the grid is considered independent of its neighbors for the purposes of training and deployment. Testing $R^{2}$ values of 0.99994 were observed for this experiment indicating a massive improvement in accuracy due to the physics-informed problem definition of the map.

Figure 19 shows results from a deployment of a hyperparameter optimized CNN framework for the same task. The best architecture was given by a four layer network with a kernel size of two. The four layers had a filter sequence of $[30,20,15,10]$. We note that this experiment now required 7 input channels to allow for the introduction of boundary information during the training of the map. We observed a similar improvement in accuracy due to the introduction of boundary information. However, we note that some inaccuracies in prediction can be observed near the boundary due to the strided nature of CNNs. These assessments utilized a zero-padding at the boundaries but more involved boundary conditions can also be embedded such as a periodic padding for each convolutional filter. Overall, the $\mathrm{CNN}$ is also able to learn the right nonlocal relationship for this closure. Our $\mathrm{CNN}$ architecture converged to a testing $R^{2}$ of 0.9967 . Once again, the effect of introducing boundary information led to much improved results.

Finally, Figure 20 shows results from a deployment of the FNN with boundary information added explicitly. Our hyperparameter search specified that the best FNN was given by a three layer 50 neuron architecture. Good results are obtained for this assessment although field values can be observed to be slightly noisy. This noise is a result of the FNN being sensitive to perturbations across the entire domain, hence small fluctuations of input variables through the domain can lead to jitter in the observed output quantities through the entire domain as well. However, despite the presence of noise, the predictions from the FNN show low deviation (particularly in the case of the parallel flux) from the true values. This might suggest a suitable use of the framework along with a low-pass spatial kernel used for postprocessing the output. We note that our neural network now constructed a map between grid variables given by an input space of dimension $256 \times 7$ and 
output space of dimension $256 \times 2$ with testing $R^{2}$ values of 0.991 .

Two-dimensional scatter plots for this experiment are also shown in Figure 21 where it is seen that all three frameworks have been improved considerably by the addition of boundary information. In addition, we also observe that the LNN framework is seen to have better test accuracy than the FNN and CNN frameworks. This is also seen in the box-plots shown in Figure 22 which assess performance for different architectures in our hyperparameter search where the LNN architectures are consistently more accurate than the CNN and FNN variants. This implies that if non-local information can be preprocessed and packaged into an input-space that still remains local, competitive and potentially superior performance may be obtained in comparison with non-local FNN and CNN architectures.

\section{Learning Hammett-Perkins}

We perform a third analysis for the Hammett-Perkins closure with 5000 fields of 256 spatial grid points in one dimension utilized as our training and validation data set. As outlined in the Guo-Tang experiments, a 'field' refers to a one-dimensional instance of multiple input and output variables defined on the entire grid. As in previous experiments, $90 \%$ of the total generated data was used for training (4500 fields) and the rest was utilized for validation and early stopping (500 fields). A separate set of 1000 fields was generated for the purpose of testing.

We start with assessments for the LNN framework shown in Figure 23. The global nature of Hammett-Perkins is immediately discernible through the performance of the trained local framework which fails to capture the right trends of the output almost entirely. This was observed for multiple different choices of the hyperparameters of the network and the results here are shown for 7 layers and 50 neurons obtained from our hyperparameter search. Note that our LNN attempts to map from a 1 dimensional input of temperature profile to a 1 dimensional output of the heat flux and we obtain very poor testing $R^{2}$ of $-5 \times 10^{-3}$.

In contrast, Figure 25 shows the results of a $\mathrm{CNN}$ with a finite non-locality where it is observed that the accuracy is improved significantly. The size of the kernel and the number of CNN layers are instrumental in the accuracy of the predictions with our hyperparameter search obtaining the best architecture with 5 hidden layers and a kernel size of 5 . The filter sequence of the best architecture is given by $[30,25,20,15,10]$. Essentially, an increase in the number of layers with a local stencil increases global influence and improves accuracy. Testing $R^{2}$ values of 0.723 are 
obtained for this particular experiment supporting the large improvement in accuracy compared to the LNN. However, we note that the global nature of Hammett-Perkins closure implies that nothing short of a FNN can obtain optimal learning.

The results of the FNN are shown in Figure 25 where a much improved performance is obtained by the best machine learning architecture obtained by a hyperparameter search. We note that the best model was given by a 1 layer, 70 neuron fully connected network. The FNN network mimics the global nature of the Fourier-space transformation and is thus able to recover the trends most accurately. A conclusion from this set of experiments is that for prediction tasks where the interaction between inputs is perfectly global, an FNN is optimally suited. However, due to the greater computational expense in training and deployment of these networks, one may also utilize a sufficiently deep CNN. The FNNs were able to obtain large testing $R^{2}$ values of 0.997 and are optimally suited for this type of closure requirement. The results of the architecture hyperparameter study for the Hammett-Perkins closure, which overwhelmingly confirms that FNNs are the dominant architecture for this globally non-local problem, is presented in the box plot of Figure 26.

To summarize the differing complexities of the proposed network architectures and closure requirements, we outline the number of trainable parameters and its effect on training and testing time for each closure learning in Table I. In addition, mean-squared errors and relative meansquared-errors for our network testing are tabulated in Table II. We note that the mean-squared error for each experiment is calculated through the following expression

$$
\operatorname{MSE}=\frac{1}{N_{t}}\left|\hat{\mathbf{y}}-\mathbf{y}^{t}\right|^{2}
$$

where $N_{t}$ corresponds to the number of test data points, $\hat{\mathbf{y}}$ is the prediction for one data point by a data-driven framework and $\mathbf{y}^{t}$ is the corresponding true value. Another similar metric is the relative mean-squared-error given by

$$
\operatorname{MSE}-\mathrm{R}=\frac{M S E}{\max \left(\mathbf{y}^{t}\right)-\min \left(\mathbf{y}^{t}\right)},
$$

Note that $\mathbf{y}^{t}$ and $\hat{\mathbf{y}}$ may be multidimensional as in the case of the Braginskii and Guo-Tang closures.

From Table I, we can clearly see that trainable parameters for FNN architectures significantly exceed those of the CNN and the LNN variants. Indeed, this is one of the reasons CNNs are prioritized for practical machine learning tasks in the image processing community. Another feature of the different networks is that FNN generally requires a much smaller training time despite having 
Table I. A tabulation of the features of the optimized architectures found after a grid-based hyperparameter search showing how the different architectures (FNN, CNN and LNN) compare with respect to (a) trainable parameters, (b) training time, (c) inference time.

\begin{tabular}{|c|c|c|c|}
\hline \multicolumn{4}{|c|}{ (a) Trainable parameters } \\
\hline & FNN & $\mathrm{CNN}$ & LNN \\
\hline Braginskii & $11,000,275$ & 59,103 & 6,003 \\
\hline Guo-Tang (Local) & 102,082 & 9,002 & 712 \\
\hline Guo-Tang (Boundary) & 120,862 & 2,637 & 20,582 \\
\hline Hammett-Perkins & 36,166 & 8,801 & 15,451 \\
\hline \multicolumn{4}{|c|}{ (b) Training time (in seconds) } \\
\hline & FNN & $\mathrm{CNN}$ & LNN \\
\hline Braginskii & 6.24 & 788.08 & 37.64 \\
\hline Guo-Tang (Local) & 8.43 & 160.63 & 42.11 \\
\hline Guo-Tang (Boundary) & 38.23 & 349.04 & 119.13 \\
\hline Hammett-Perkins & 14.41 & 51.47 & 73.59 \\
\hline \multicolumn{4}{|c|}{ (c) Testing time (in seconds) } \\
\hline & FNN & CNN & LNN \\
\hline Braginskii & 11.65 & 13.03 & 14.50 \\
\hline Guo-Tang (Local) & 0.49 & 0.69 & 6.87 \\
\hline Guo-Tang (Boundary) & 0.40 & 0.57 & 7.92 \\
\hline Hammett-Perkins & 0.27 & 0.92 & 12.13 \\
\hline
\end{tabular}

a much greater number of trainable parameters. We attribute this to the fact that the higher dimensional optimization inherent in having more decision variables in the objective function leads to a very complicated loss surface with many local minima. This implies that an optimization converges very quickly but is stuck at a sub-optimal location of a response surface, leading to lower accuracy on some models. The relative mean-squared-errors in Table II indicate that the best architectures are seen to approach ideal single-precision accuracy. This point is noteworthy, since efficient deployments of neural networks using platforms such as TensorFlow are commonly associated with single precision operations. 
Table II. A tabulation of the features of the optimized architectures found after a grid-based hyperparameter search showing how the different architectures (FNN, CNN and LNN) compare with respect to (a) meansquared error and (b) relative mean-squared-error for our different requirements. The asterisked quantity refers to the accuracy on the extrapolation data set assessed in Section V D.

\begin{tabular}{|c|c|c|c|}
\hline \multicolumn{4}{|c|}{ (a) Testing mean-squared-error } \\
\hline & FNN & CNN & LNN \\
\hline Braginskii & $3.26 \times 10^{-3}$ & $2.19 \times 10^{-4}$ & $1.13 \times 10^{-5}$ \\
\hline Guo-Tang (Local) & $9.03 \times 10^{-2}$ & $1.44 \times 10^{-1}$ & $1.48 \times 10^{-1}$ \\
\hline Guo-Tang (Boundary) & $1.10 \times 10^{-3}$ & $3.70 \times 10^{-4}$ & $1.28 \times 10^{-5}$ \\
\hline Hammett-Perkins & $4.67 \times 10^{-4}$ & $2.63 \times 10^{-2}$ & $1.97 \times 10^{-1}$ \\
\hline Hammett-Perkins* & $2.43 \times 10^{-3}$ & $6.70 \times 10^{-2}$ & $2.30 \times 10^{-2}$ \\
\hline (a) Testing relative mean-squared-error \\
\hline & DNN & CNN & LNN \\
\hline Braginskii & $8.61 \times 10^{-3}$ & $5.78 \times 10^{-4}$ & $2.98 \times 10^{-5}$ \\
\hline Guo-Tang (Local) & $3.11 \times 10^{-2}$ & $4.96 \times 10^{-2}$ & $5.09 \times 10^{-2}$ \\
\hline Guo-Tang (Boundary) & $3.78 \times 10^{-4}$ & $1.28 \times 10^{-4}$ & $4.41 \times 10^{-6}$ \\
\hline Hammett-Perkins & $1.19 \times 10^{-4}$ & $6.69 \times 10^{-3}$ & $5.01 \times 10^{-2}$ \\
\hline Hammett-Perkins* & $2.93 \times 10^{-3}$ & $8.14 \times 10^{-2}$ & $2.79 \times 10^{-2}$ \\
\hline
\end{tabular}

\section{A note on extrapolation}

In the previous sections, we have demonstrated the applicability of learned surrogates when subject to the domain of the training data. In practice, one may have a lot of data to learn from but that data may not have a broad span of a system's parameter space to generate training data; this may be considered as a balance between big data, i.e. a large quantity, and broad data, a large and statistically meaningful quantity of data. Thus, predictions may have to be extrapolated based on what was learnt from the available training domain. Before presenting outcomes of a closure surrogate in an extrapolating regime, we wish to note that extrapolating outside the training domain using a learned neural network architecture is a non-trivial task that requires careful application. A simple demonstrative example of the unreliability of trying to emulate even the most simple of functions, the identity $f(x)=x$, was presented recently ${ }^{26}$. While an exact analytic solution 
of network coefficients can be found for a simple network architecture like the identify function, to allow accurate extrapolation outside a training domain, Gin et al. ${ }^{26}$ highlight the sensitivity of learned network coefficients and thus the reliability of predictions outside the training domain even for such a simple problem. We believe this is an important lesson to keep in mind when applying neural network methods to real problems, and emphasizes that a network may learn to mimic behaviour of a set of training data but cannot reliably learn the underlying general functional relationships relating input and output quantities.

One of the key challenges of machine learning is to detect extrapolation. Machine learning frameworks are notoriously unreliable in extrapolating regimes as mentioned previously. Detecting extrapolation is useful for determining if more data should be generated and the framework should be retrained using this new data before deployment. For example, we outline the performance of the FNN obtained for the Hammett-Perkins closure surrogate in the previous section for a data point which is generated from a slightly different underlying distribution and the result is shown in Figure 27. It can be seen that the true profile has more high frequency content which the trained network struggles to predict even though low frequency trends are recovered somewhat. The PDF of the true dataset is also recovered approximately (note how this new data set is distinctly multi-modal). The scatter plots also show consistent errors through the entirety of the domain. Quantitative assessments of the drop in performance during extrapolation are also outlined in Table II where an entry in the Hammett-Perkins closure section indicates that the testing MSE is higher for this inference.

Finally, we outline the use of T-distributed Stochastic Neighbor Embedding (t-SNE) ${ }^{27}$ which is a nonlinear embedding of our training and testing data onto a two-dimensional space. The embedding only requires the input data (i.e., the target values need not be known) and can be a check to determine the risk of extrapolation. Figure 28 shows this analysis on the new extrapolative dataset with the multimodal distribution of outputs. Two distinct clusters emerge corresponding to the two distinct distributions in our total dataset. Note however, that this method cannot be coupled with a computational deployment of a closure as it is significantly costly in comparison with a forward pass through any of these networks. However, it can be a useful diagnostic to determine if a machine learning framework has contributed to failure of a simulation. 


\section{CONCLUSION}

From this study we have found there is utility in choosing a neural surrogate closure's network architecture based on the physics expected in the chosen problem. A simple schematic summary of this is shown Fig. 29, demonstrating that, for a fixed amount of training data, the relative performance of different network architectures varies with the spatial locality of the target closure.

In the simplest limit is local closure, where point-wise values of closed quantities can be expressed as a functions of macroscopic values at the same points. The primary example of this regime used in this work was the Braginskii closure for a collisional magnetized plasma, in which velocity-space equilibration through collisions sets up a local closure. In this scenario, we observed that a simple local network clearly outperformed the more complex CNN and FNN network architectures. The LNN architecture robustly and efficiently reproduced the required physics in high dimensional simulations without the excessive computational burden more general connected network architectures would produce ${ }^{15}$.

Next, one can consider intermediate regimes where non-locality exists but is contained over a finite range of influence. This concept has notably been discussed by Hazeltine ${ }^{6}$ in the context of source and boundary edge properties influencing the dynamics of the bulk plasma. In this scenario, we observe that physics-informed local networks with boundary data generally performed the best. However the margin of improvement compared to the nearest competing architecture, $\mathrm{CNN}$, is not as large compared to the other two extremes discussed in this study. We therefore recommend that both physics-informed local networks and CNN architectures, similar to that commonly used in image processing where there is a relationship between neighbouring points, can yield a good balance between reproducing the required non-local physics, without an FNN.

Finally, in the extreme of a globally non-local closure, such as in the case in which collisionlessphase-mixing ${ }^{15,16,28,29}$ provides a truly global influence on point-wise values, we find that a FNN is clearly required to emulate the global non-locality of this style of closure, with no competing architecture rivaling the accuracy capable of FNNs.

Our insights from this article suggest that the effective development of data-driven closures for plasma physics applications require embedded physical intuition, thorough validation for generalization and cognizance of the dangers of extrapolation. Our future endeavors, will build on our conclusions for this work to develop closures that port fidelity from 6D simulations to fluid plasma simulations effectively. 


\section{ACKNOWLEDGMENTS}

We acknowledge productive discussions with Dr. Sandeep Madireddy and Dr. Bethany Lusch for this article. This material is based upon work supported by the U.S. Department of Energy (DOE), Office of Science, Office of Advanced Scientific Computing Research, under Contract DE-AC02-06CH11357. This research was funded in part and used resources of the Argonne Leadership Computing Facility, which is a DOE Office of Science User Facility supported under Contract DE-AC02-06CH11357. Part of this work was performed at Los Alamos National Laboratory (contract No. 89233218CNA000001) with support from the SciDAC project on Tokamak Disruption Simulation (TDS) by Office of Fusion Energy Science and Office of Advanced Scientific Computing and by the Los Alamos National Laboratory LDRD program under project number 20180756PRD4.

\section{DATA AVAILABILITY STATEMENT}

The data that support the findings of this study are available from the corresponding author upon reasonable request.

\section{REFERENCES}

${ }^{1} \mathrm{~F}$. Chen, Introduction to plasma physics and controlled fusion. Third edition. (Springer International Publishing, 2016).

${ }^{2}$ S. I. Braginskii, “Transport Processes in a Plasma,” Reviews of Plasma Physics 1, 205 (1965).

${ }^{3}$ P. Helander and D. Sigmar, Collisional Transport in Magnetized Plasmas, Cambridge Monographs on Plasma Physics (Cambridge University Press, 2005).

${ }^{4}$ W. Park, E. V. Belova, G. Y. Fu, X. Z. Tang, H. R. Strauss, and L. E. Sugiyama, "Plasma simulation studies using multilevel physics models," Physics of Plasmas 6, 1796-1803 (1999), https://doi.org/10.1063/1.873437.

${ }^{5}$ E. D. Held, J. D. Callen, C. C. Hegna, C. R. Sovinec, T. A. Gianakon, and S. E. Kruger, "Nonlocal closures for plasma fluid simulations," Physics of Plasmas 11, 2419-2426 (2004), https://doi.org/10.1063/1.1645520.

${ }^{6}$ R. D. Hazeltine, "Transport theory in the collisionless limit," Physics of Plasmas 5, 3282-3286 (1998), https://doi.org/10.1063/1.872996. 
${ }^{7}$ S. Yarlanki, B. Rajendran, and H. Hamann, "Estimation of turbulence closure coefficients for data centers using machine learning algorithms," in 13th InterSociety Conference on Thermal and Thermomechanical Phenomena in Electronic Systems (2012) pp. 38-42.

${ }^{8}$ C.-W. Chang and N. T. Dinh, "Classification of machine learning frameworks for data-driven thermal fluid models,” International Journal of Thermal Sciences 135, 559 - 579 (2019).

${ }^{9}$ O. San and R. Maulik, "Machine learning closures for model order reduction of thermal fluids," Applied Mathematical Modelling 60, 681 - 710 (2018).

${ }^{10}$ K. Montes, C. Rea, R. Granetz, R. Tinguely, N. Eidietis, O. Meneghini, D. Chen, B. Shen, B. Xiao, K. Erickson, and M. Boyer, "Machine learning for disruption warnings on alcator c-mod, DIII-d, and EAST," Nuclear Fusion 59, 096015 (2019).

${ }^{11}$ J. Kates-Harbeck, A. Svyatkovskiy, and W. Tang, "Predicting disruptive instabilities in controlled fusion plasmas through deep learning," Nature (London) 568 (2019), 10.1038/s41586019-1116-4.

${ }^{12}$ D. Wroblewski, G. Jahns, and J. Leuer, "Tokamak disruption alarm based on a neural network model of the high- beta limit," Nuclear Fusion 37, 725-741 (1997).

${ }^{13}$ D. Rastovic, "Targeting and synchronization at tokamak with recurrent artificial neural networks,” Neural Computing and Applications 21, 1065-1069 (2012).

${ }^{14}$ E. Coccorese, R. Martone, and F. C. Morabito, "A neural network approach for the solution of electric and magnetic inverse problems," IEEE Transactions on Magnetics 30, 2829-2839 (1994).

${ }^{15}$ C. Ma, B. Zhu, X.-Q. Xu, and W. Wang, "Machine learning surrogate models for landau fluid closure," Physics of Plasmas 27, 042502 (2020), https://doi.org/10.1063/1.5129158.

${ }^{16}$ G. W. Hammett and F. W. Perkins, "Fluid moment models for Landau damping with application to the ion-temperature-gradient instability," Phys. Rev. Lett. 64, 3019-3022 (1990).

${ }^{17}$ Z. Guo and X.-Z. Tang, "Parallel Heat Flux from Low to High Parallel Temperature along a Magnetic Field Line,” Phys. Rev. Lett. 108, 165005 (2012).

${ }^{18} \mathrm{~J}$. Bedrossian and N. Masmoudi, "Inviscid damping and the asymptotic stability of planar shear flows in the 2d euler equations," (2013), arXiv:1306.5028 [math.AP].

${ }^{19}$ P. J. Morrison, "Hamiltonian description of vlasov dynamics: Action-angle variables for the continuous spectrum," Transport Theory and Statistical Physics 29, 397-414 (2000), https://doi.org/10.1080/00411450008205881. 
${ }^{20}$ J. M. Heninger and P. J. Morrison, “An integral transform technique for kinetic systems with collisions," Phys. Plasmas 25, 082118 (2018).

${ }^{21}$ Z. Guo and X.-Z. Tang, "Parallel transport of long mean-free-path plasma along open magnetic field lines: Parallel heat flux," Physics of Plasmas (1994-present) 19, 062501 (2012).

${ }^{22}$ A. Krizhevsky, I. Sutskever, and G. E. Hinton, "Imagenet classification with deep convolutional neural networks," in Advances in neural information processing systems (2012) pp. 1097-1105.

${ }^{23}$ R. Maulik and O. San, "A neural network approach for the blind deconvolution of turbulent flows," Journal of Fluid Mechanics 831, 151-181 (2017).

${ }^{24}$ M. Abadi, A. Agarwal, P. Barham, E. Brevdo, Z. Chen, C. Citro, G. S. Corrado, A. Davis, J. Dean, M. Devin, S. Ghemawat, I. Goodfellow, A. Harp, G. Irving, M. Isard, Y. Jia, R. Jozefowicz, L. Kaiser, M. Kudlur, J. Levenberg, D. Mané, R. Monga, S. Moore, D. Murray, C. Olah, M. Schuster, J. Shlens, B. Steiner, I. Sutskever, K. Talwar, P. Tucker, V. Vanhoucke, V. Vasudevan, F. Viégas, O. Vinyals, P. Warden, M. Wattenberg, M. Wicke, Y. Yu, and X. Zheng, "TensorFlow: Large-scale machine learning on heterogeneous systems," (2015), software available from tensorflow.org.

${ }^{25}$ D. P. Kingma and J. Ba, "Adam: A method for stochastic optimization," (2014), arXiv:1412.6980 [cs.LG].

${ }^{26}$ C. Gin, B. Lusch, S. L. Brunton, and J. N. Kutz, "Deep learning models for global coordinate transformations that linearize pdes," arXiv preprint arXiv:1911.02710 (2019).

${ }^{27}$ G. E. Hinton and S. T. Roweis, "Stochastic neighbor embedding," in Advances in neural information processing systems (2003) pp. 857-864.

${ }^{28}$ G. W. Hammett, W. Dorland, and F. W. Perkins, "Fluid models of phase mixing, landau damping, and nonlinear gyrokinetic dynamics," Physics of Fluids B: Plasma Physics 4, 2052-2061 (1992), https://doi.org/10.1063/1.860014.

${ }^{29}$ D. Tsiklauri and T. Haruki, "Physics of collisionless phase mixing," Physics of Plasmas 15, 112902 (2008), https://doi.org/10.1063/1.3023157.

${ }^{30}$ M. Raissi, P. Perdikaris, and G. Karniadakis, "Physics-informed neural networks: A deep learning framework for solving forward and inverse problems involving nonlinear partial differential equations," Journal of Computational Physics 378, 686 - 707 (2019).

${ }^{31}$ J.-L. Wu, H. Xiao, and E. Paterson, "Physics-informed machine learning approach for augmenting turbulence models: A comprehensive framework," Phys. Rev. Fluids 3, 074602 (2018). 


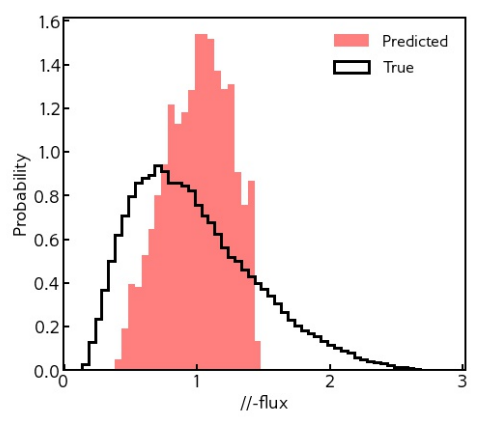

(a)

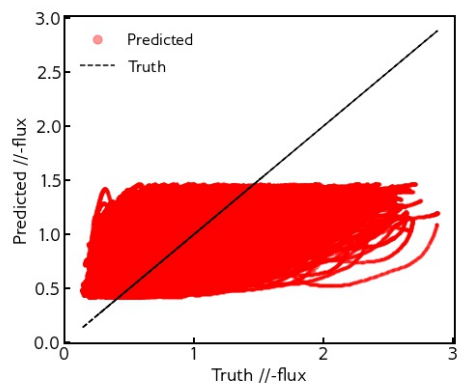

(c)

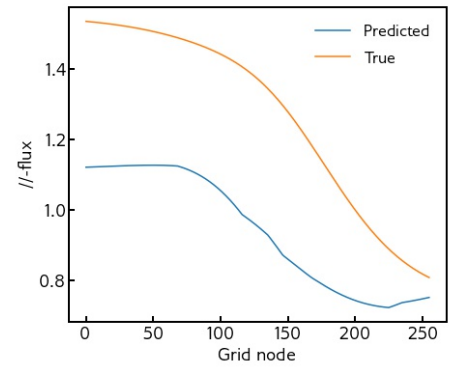

(e)

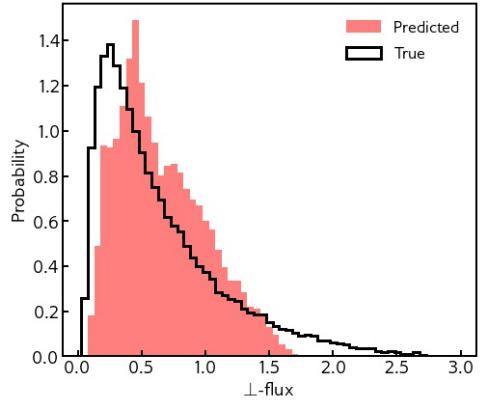

(b)

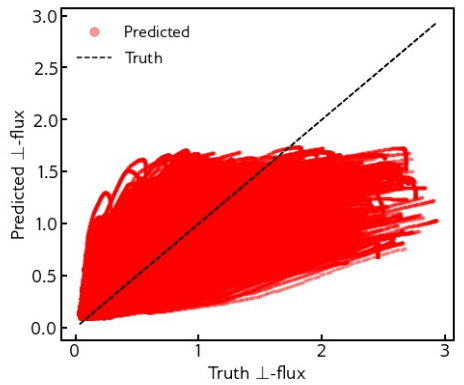

(d)

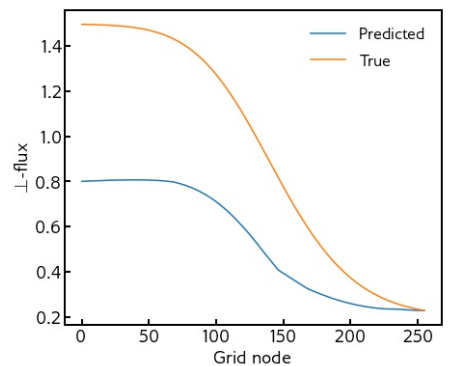

(f)

Figure 13. Machine learning results for learning the Guo-Tang closure with no boundary information for the locally connected machine learning framework. The top row (a) and (b) shows PDFs of the true and predicted parallel and perpendicular fluxes, the middle row (c) and (d) shows the scatter plots for the same fluxes, while the bottom row (e) and (f) shows a sample field prediction by the trained frameworks. All assessments are on the test data set. 


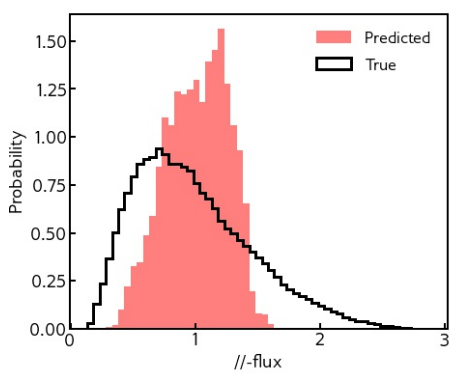

(a)

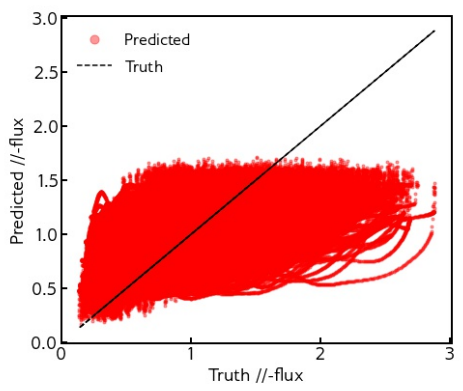

(c)

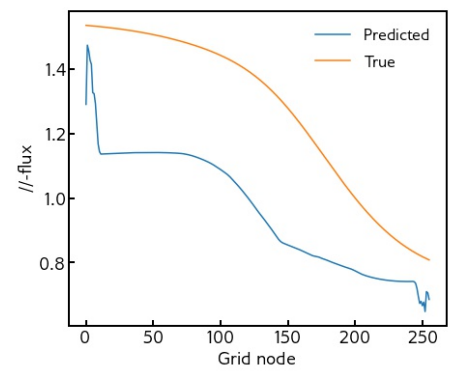

(e)

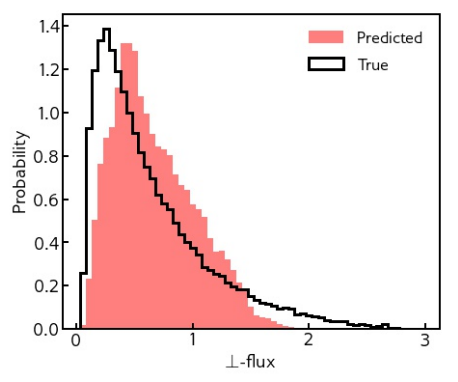

(b)

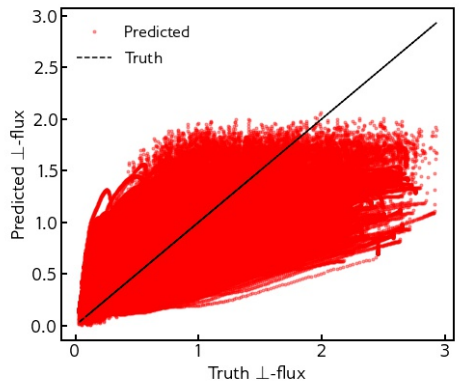

(d)

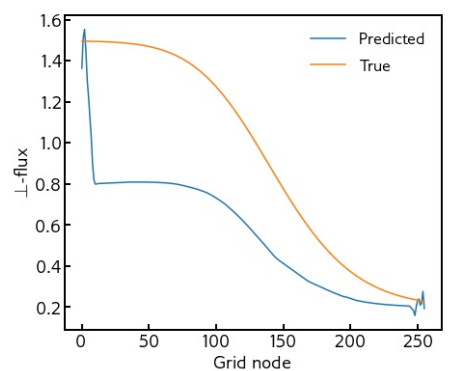

(f)

Figure 14. Machine learning results for learning the Guo-Tang closure with no boundary information for the CNN learning framework. The top row (a) and (b) shows PDFs of the true and predicted parallel and perpendicular fluxes, the middle row (c) and (d) shows the scatter plots for the same fluxes, while the bottom row (e) and (f) shows a sample field prediction by the trained frameworks. All assessments are on the test data set. 


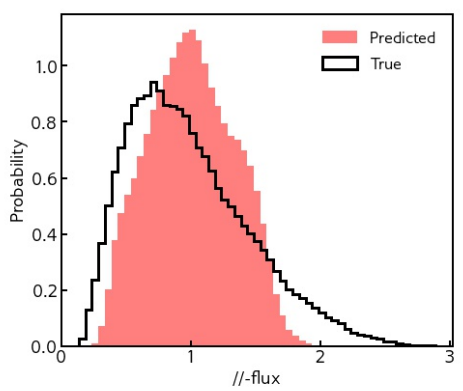

(a)

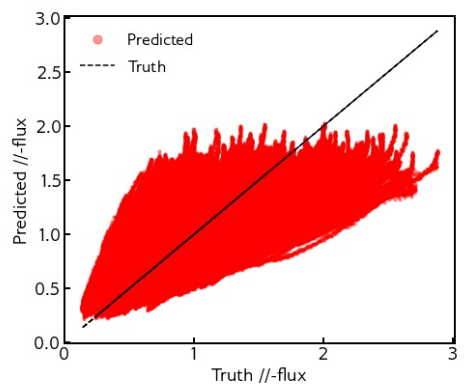

(c)

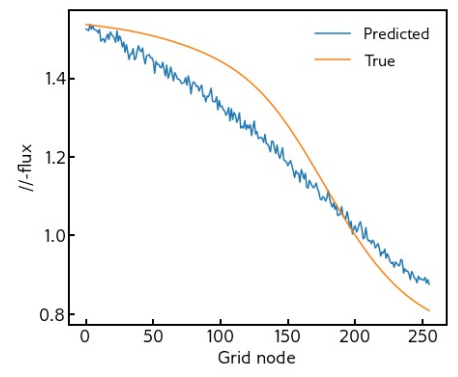

(e)

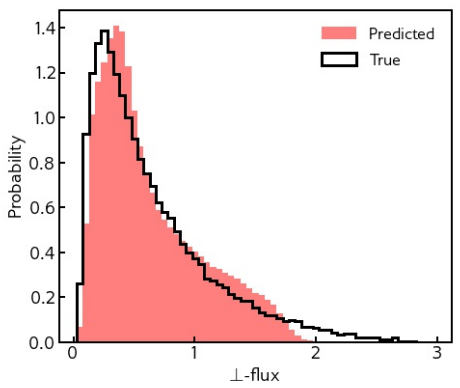

(b)

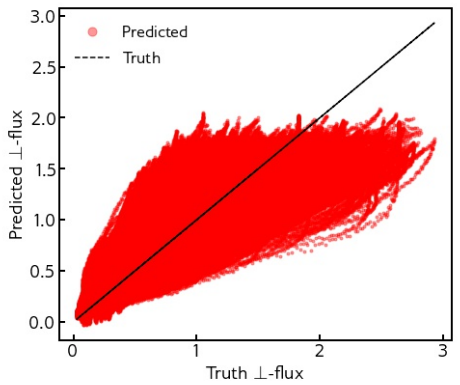

(d)

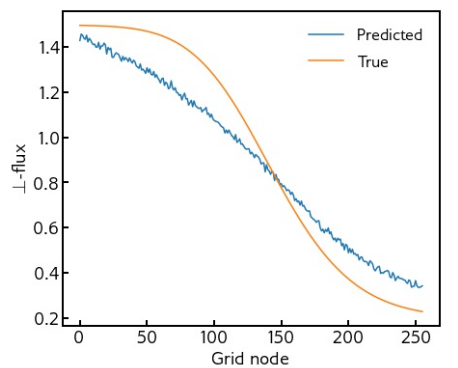

(f)

Figure 15. Machine learning results for learning the Guo-Tang closure with no boundary information for the FNN framework. The top row (a) and (b) shows PDFs of the true and predicted parallel and perpendicular fluxes, the middle row (c) and (d) shows the scatter plots for the same fluxes, while the bottom row (e) and (f) shows a sample field prediction by the trained frameworks. All assessments are on the test data set. 


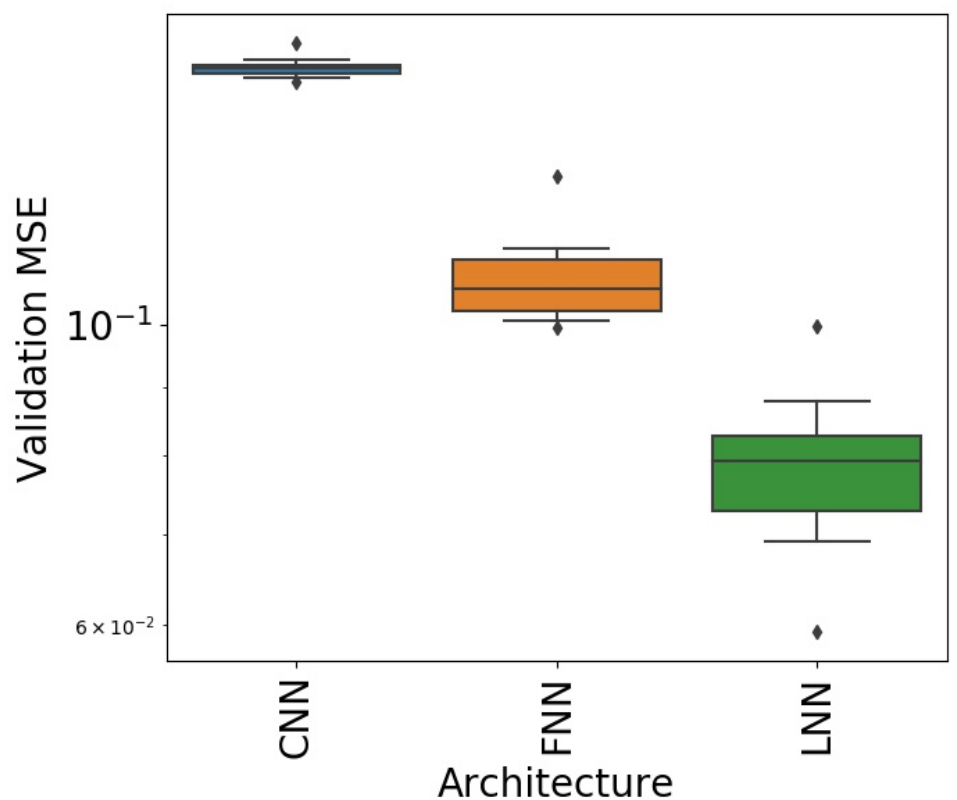

Figure 16. A grid-based hyperparameter search comparing accuracies for the LNN, CNN and FNN architectures for learning the Guo-Tang closure with local information only. The box plots are shown for validation mean-squared errors. The whiskers indicate the $5^{\text {th }}$ and $95^{\text {th }}$ percentile of evaluated models. The edges of the box indicate the $25^{\text {th }}$ and $75^{\text {th }}$ percentiles and the line within a box indicates the median value. The LNN performs better than the other architectures on the training data set for this closure but the absence of boundary information leads to poor performance across architectures. 


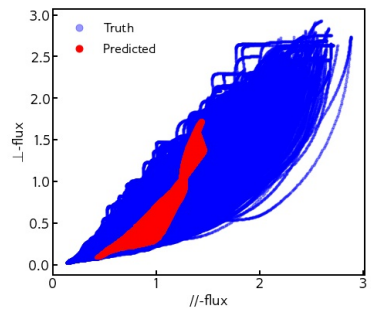

(a)LNN

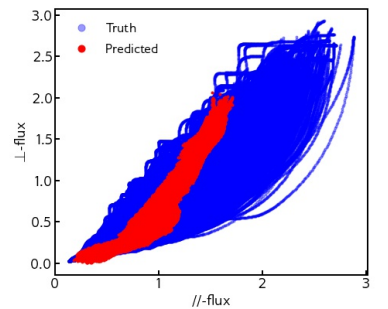

(b) $\mathrm{CNN}$

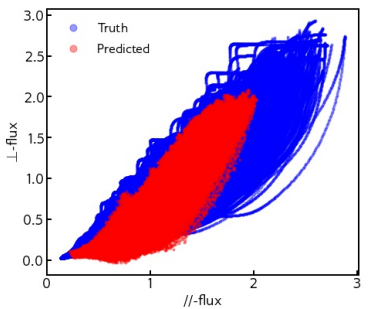

(c)FNN

Figure 17. Scatter plots for true and predicted parallel versus perpendicular fluxes of the Guo-Tang closure with solely local information for (a) LNN, (b) CNN, and (c) FNN architectures. For the Guo-Tang closure with solely local information, the FNN architecture is seen to perform the best on testing data. 


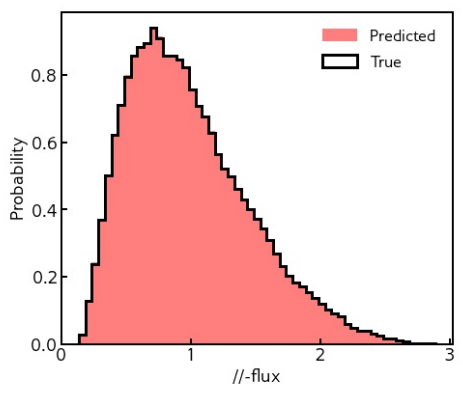

(a)

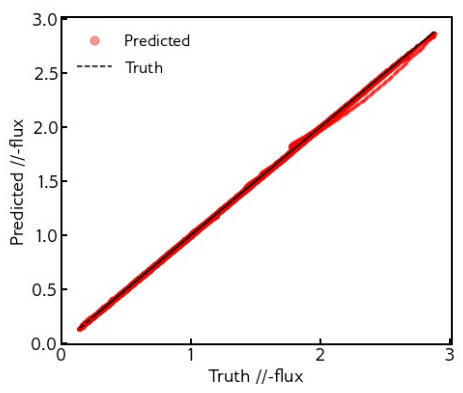

(c)

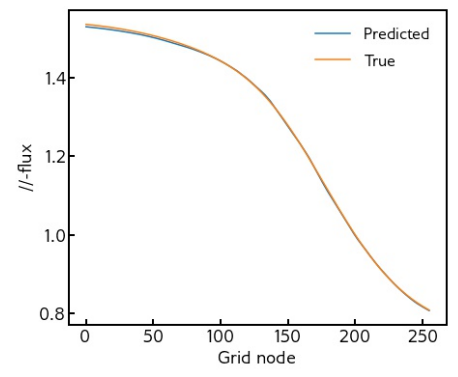

(e)

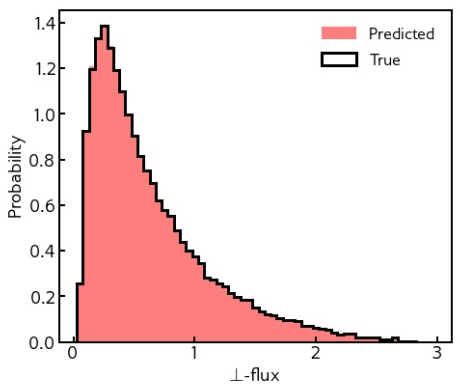

(b)

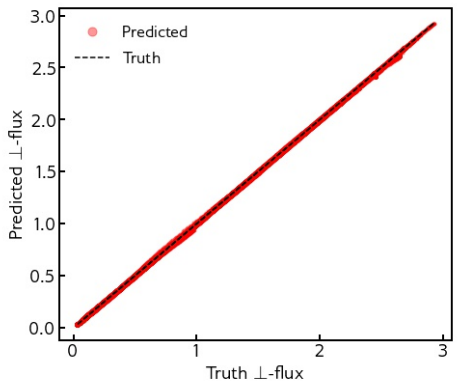

(d)

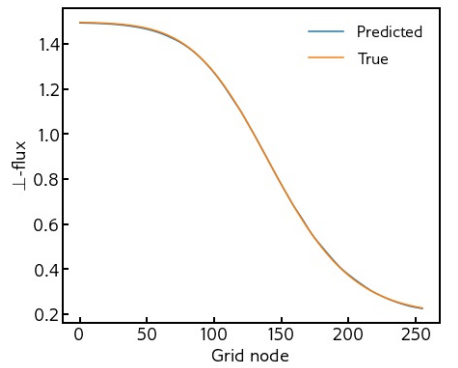

(f)

Figure 18. Machine learning results for learning the Guo-Tang closure for the LNN framework with inputs augmented by boundary information. The top row (a) and (b) shows PDFs of the true and predicted parallel and perpendicular fluxes, the middle row (c) and (d) shows the scatter plots for the same fluxes, while the bottom row (e) and (f) shows a sample field prediction by the trained frameworks. All assessments are on the test data set. 


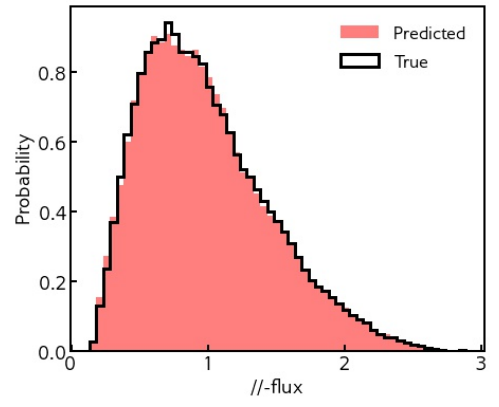

(a)

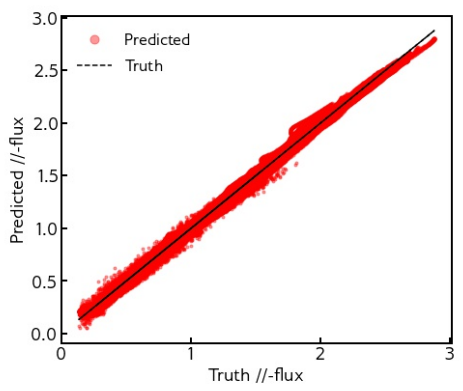

(c)

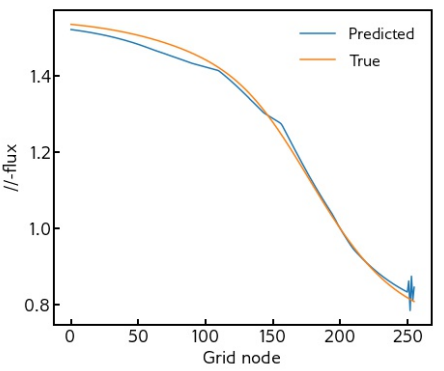

(e)

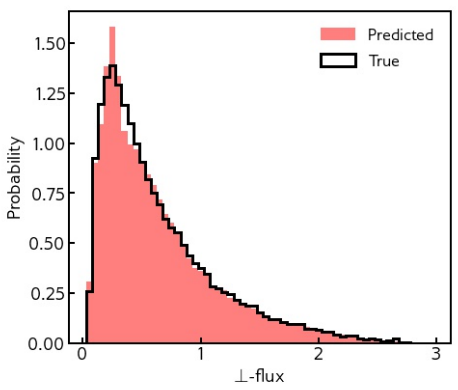

(b)

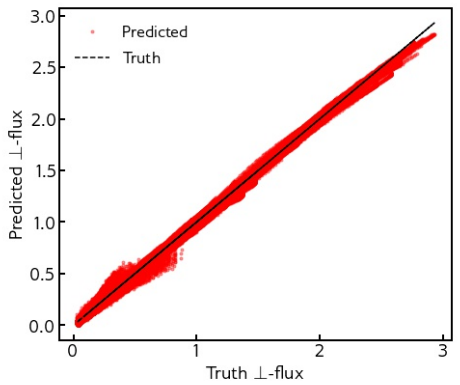

(d)

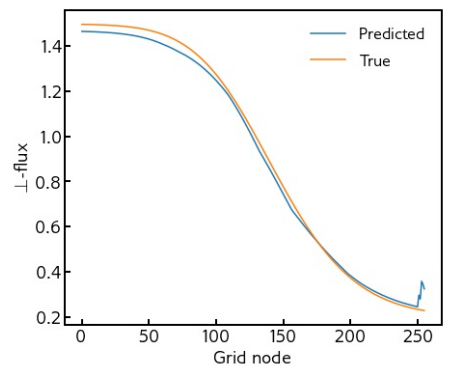

(f)

Figure 19. Machine learning results for learning the Guo-Tang closure for the CNN framework with inputs augmented by boundary information. The top row (a) and (b) shows PDFs of the true and predicted parallel and perpendicular fluxes, the middle row (c) and (d) shows the scatter plots for the same fluxes, while the bottom row (e) and (f) shows a sample field prediction by the trained frameworks. All assessments are on the test data set. 


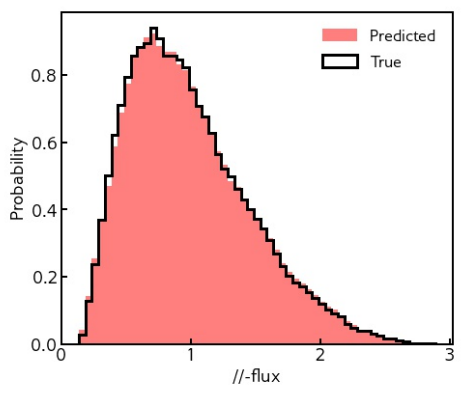

(a)

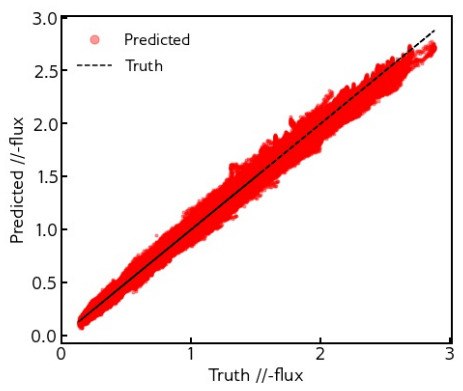

(c)

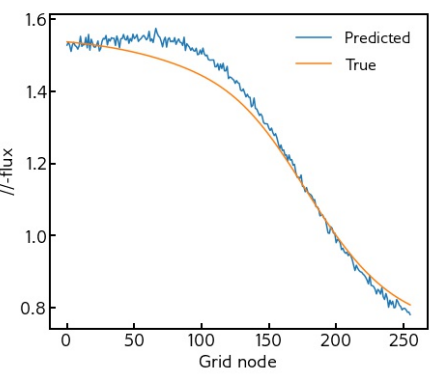

(e)

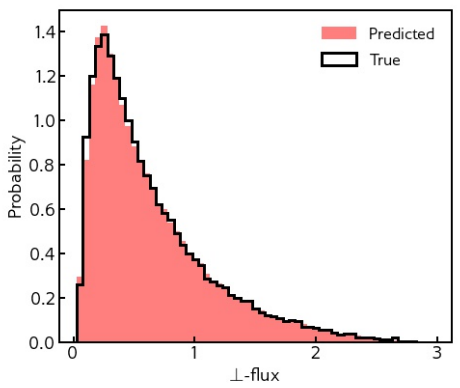

(b)

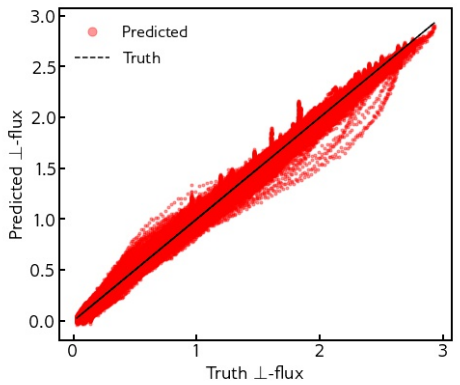

(d)

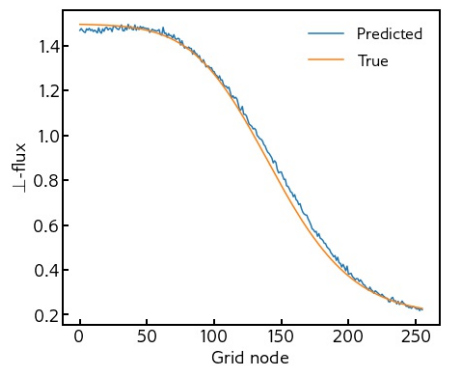

(f)

Figure 20. Machine learning results for learning the Guo-Tang closure for the FNN framework with inputs augmented by boundary information. The top row (a) and (b) shows PDFs of the true and predicted parallel and perpendicular fluxes, the middle row (c) and (d) shows the scatter plots for the same fluxes, while the bottom row (e) and (f) shows a sample field prediction by the trained frameworks. All assessments are on the test data set. 


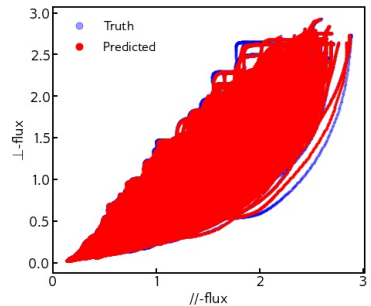

(a)LNN

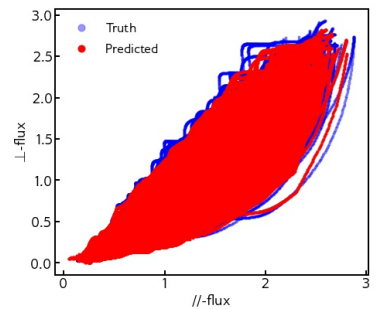

(b) $\mathrm{CNN}$

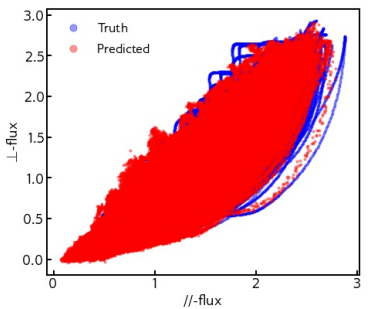

(c) $\mathrm{CNN}$

Figure 21. Scatter plots for true and predicted parallel versus perpendicular fluxes of the Guo-Tang closure with global information for (a) LNN, (b) CNN, and (c) FNN architectures. For the Guo-Tang closure with global information, all architectures are able to recover trends in the true testing data. 


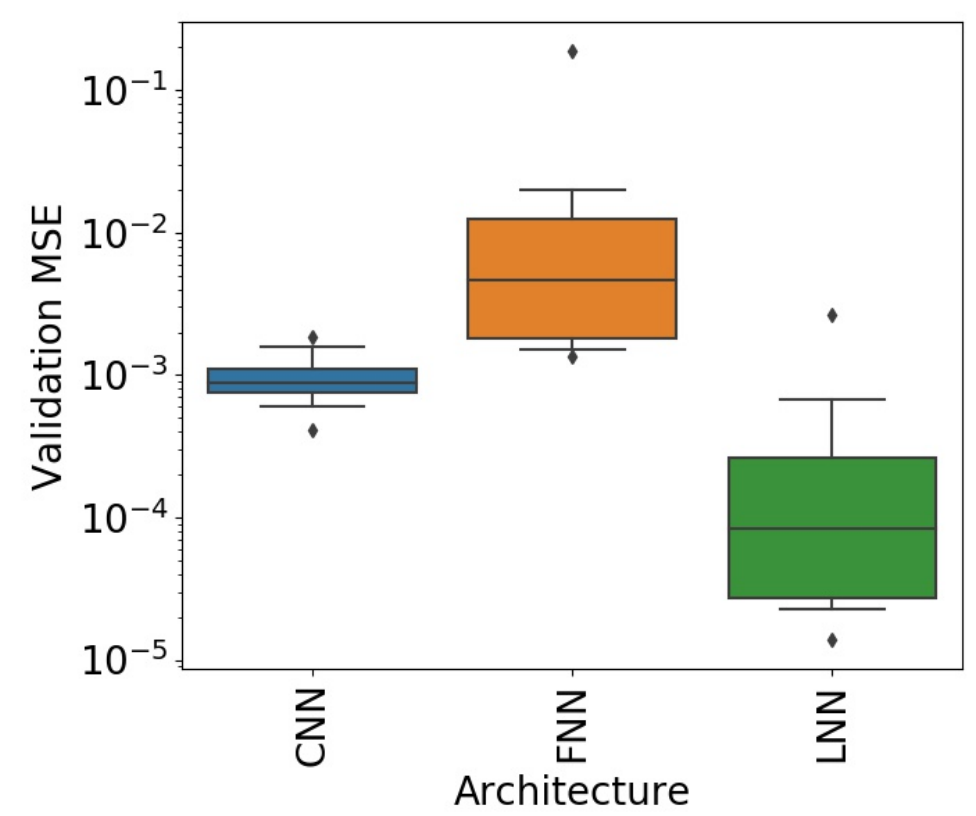

Figure 22. A grid-based hyperparameter search comparing accuracies for the LNN, CNN and FNN architectures for learning the Guo-Tang closure with global information. The box plots are shown for validation mean-squared errors. The whiskers indicate the $5^{\text {th }}$ and $95^{\text {th }}$ percentile of evaluated models. The edges of the box indicate the $25^{\text {th }}$ and $75^{\text {th }}$ percentiles and the line within a box indicates the median value. It is apparent that the FNN performs better than the other architectures for this closure. 


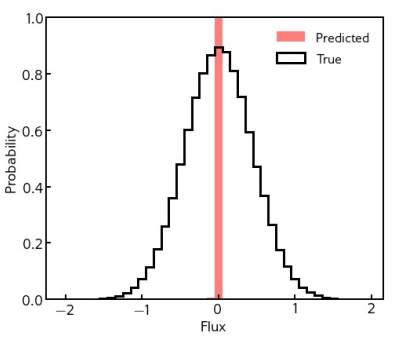

(a)

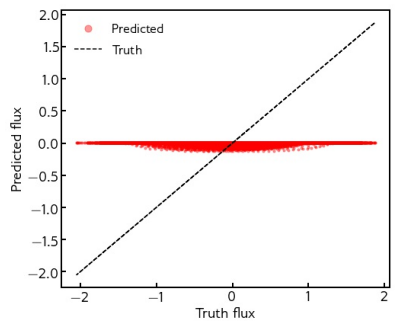

(b)

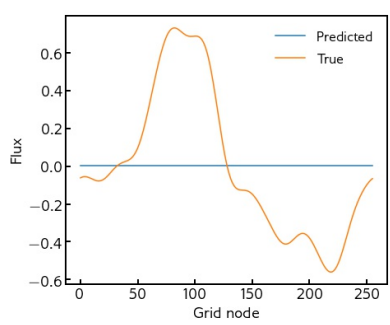

(c)

Figure 23. Results from machine learning the Hammett-Perkins closure with (a) PDFs, (b) scatter plots, and (c) a sample prediction visualized against the truth for the LNN. These are generated from the testing data set.

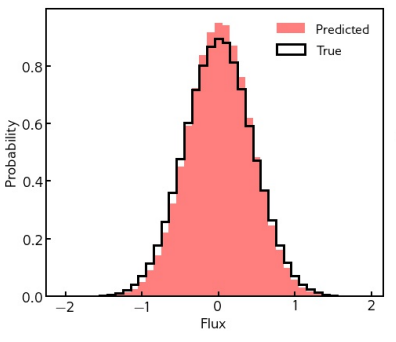

(a)

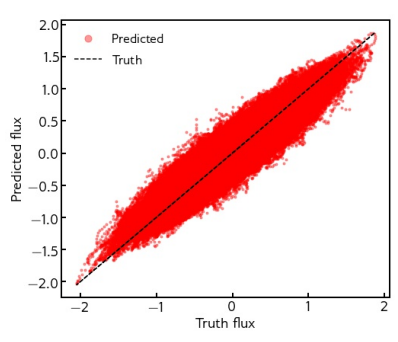

(b)

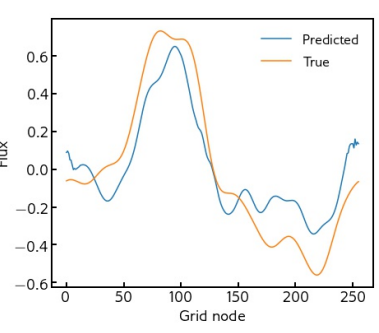

(c)

Figure 24. Results from machine learning the Hammett-Perkins closure with (a) PDFs, (b) scatter plots, and (c) a sample prediction visualized against the truth for the $\mathrm{CNN}$. These are generated from the testing data set. 


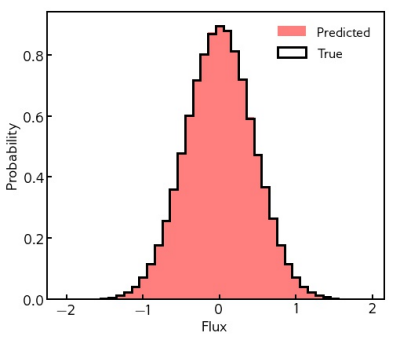

(a)

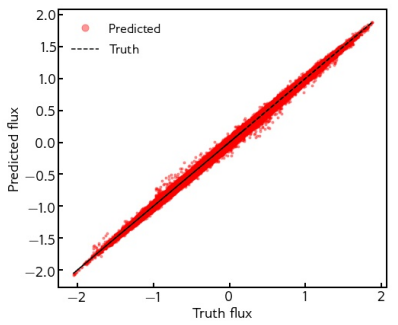

(b)

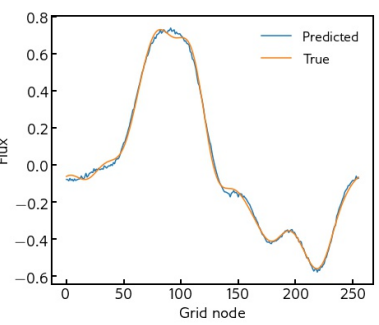

(c)

Figure 25. Results from machine learning the Hammett-Perkins closure with (a) PDFs, (b) scatter plots, and (c) a sample prediction visualized against the truth for the FNN. These are generated from the testing data set. 


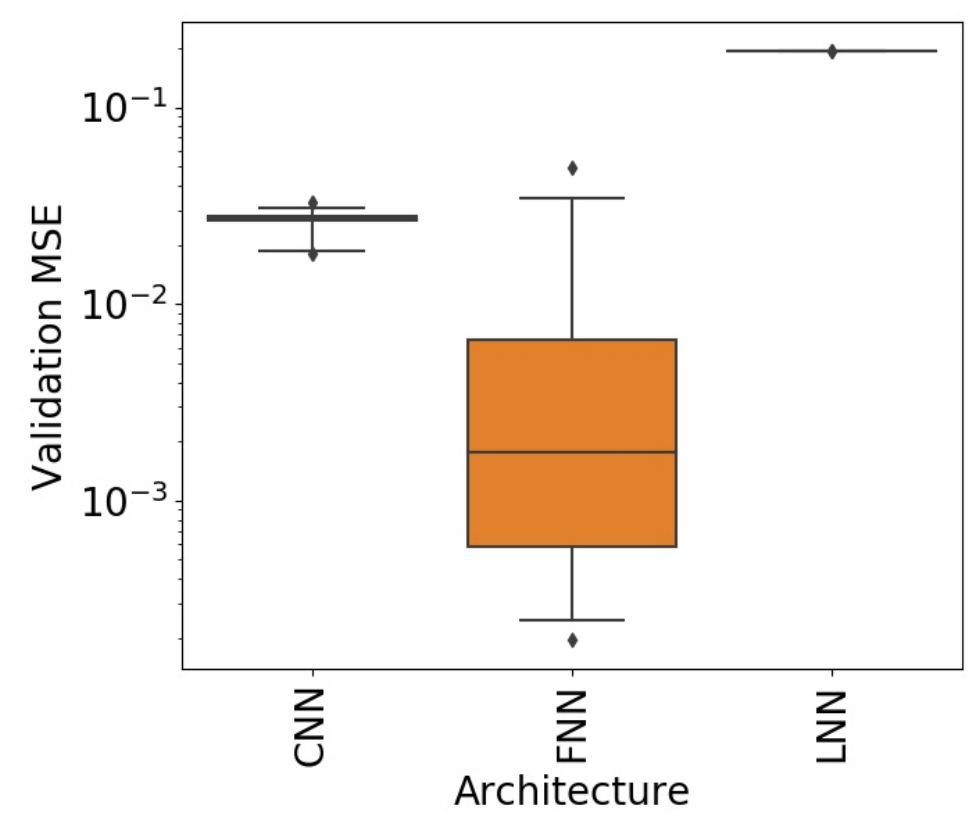

Figure 26. A grid-based hyperparameter search comparing accuracies for the LNN, CNN and FNN architectures trained for the Hammett-Perkins test case. The box plots are shown for validation mean-squared errors. The whiskers indicate the $5^{\text {th }}$ and $95^{\text {th }}$ percentile of evaluated models. The edges of the box indicate the $25^{\text {th }}$ and $75^{\text {th }}$ percentiles and the line within a box indicates the median value. It is apparent that the FNN performs well for this problem, while other architectures struggle. 


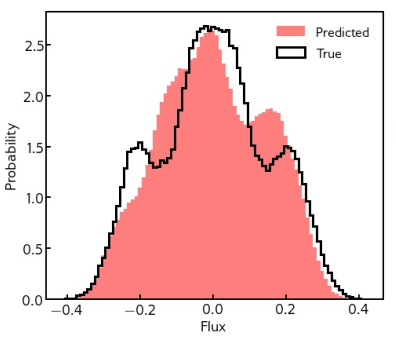

(a)

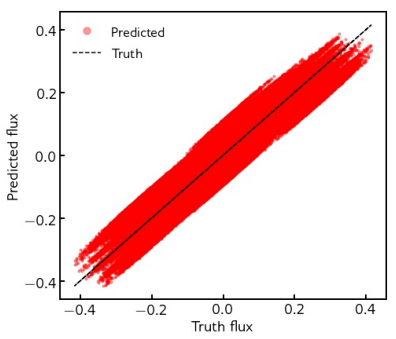

(b)

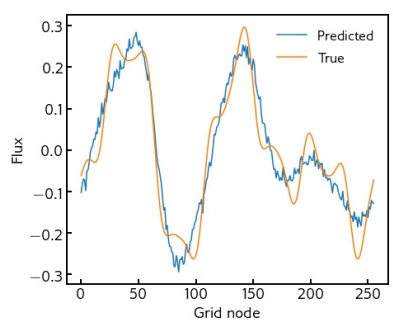

(c)

Figure 27. Extrapolative results from machine learning the Hammett-Perkins closure with (a) PDFs, (b) scatter plots, and (c) a sample prediction visualized against the truth for the FNN. These are generated for the testing data set from a different underlying distribution. Results of extrapolation outside the space of trained input data can be clearly observed with lesser accuracy in PDF prediction.

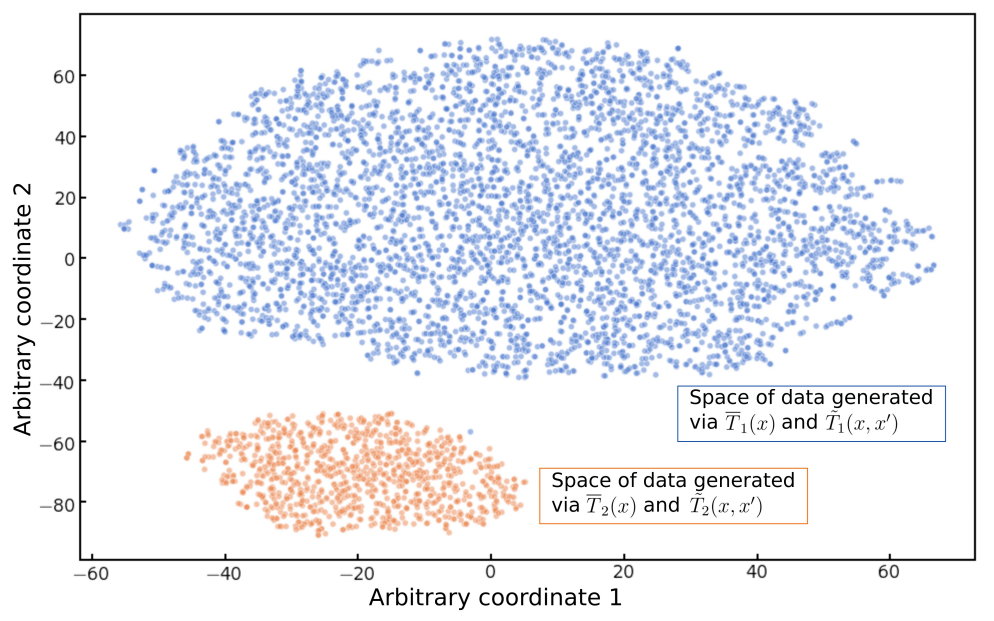

Figure 28. T-SNE encoding of training and testing datasets from different underlying distributions. The two clusters indicate two distinct distributions between training and testing. Formation of distinct clusters in input data is a key indicator of operating in an extrapolating fashion, in which care must be taken. 


\begin{tabular}{|c|c|c|c|}
\hline $\begin{array}{c}\text { Regimes of } \\
\text { locality to } \\
\text { inform closure }\end{array}$ & 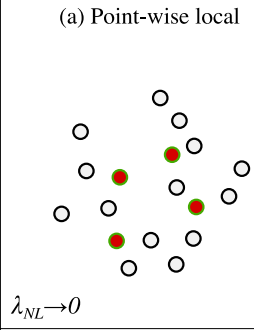 & (b) Finite non-locality & (c) Global non-locality \\
\hline $\begin{array}{c}\text { Suitable surrogate } \\
\text { network } \\
\text { architectures }\end{array}$ & $\begin{array}{l}\text { Locally connected } \\
\text { network }\end{array}$ & $\begin{array}{l}\text { Physics-informed local network } \\
\text { Convolutional NN }\end{array}$ & $\begin{array}{l}\text { Fully connected } \\
\text { network }\end{array}$ \\
\hline
\end{tabular}

Figure 29. Simple schematic of varying classes of closure formulations. In each figure a red focus point can be thought to have a non-local interaction length, $\lambda_{N L}$, bounded by the dashed green line containing blue points that influence the dynamics of a given focus point. This interaction region can be qualified in each region as (a) $\lambda_{N L} \rightarrow 0$ and local information is sufficient, (b) finite $\lambda_{N L}$ and finite region of interaction, and (c) $\lambda_{N L} \rightarrow \infty$ and the entire global domain must be considered. 


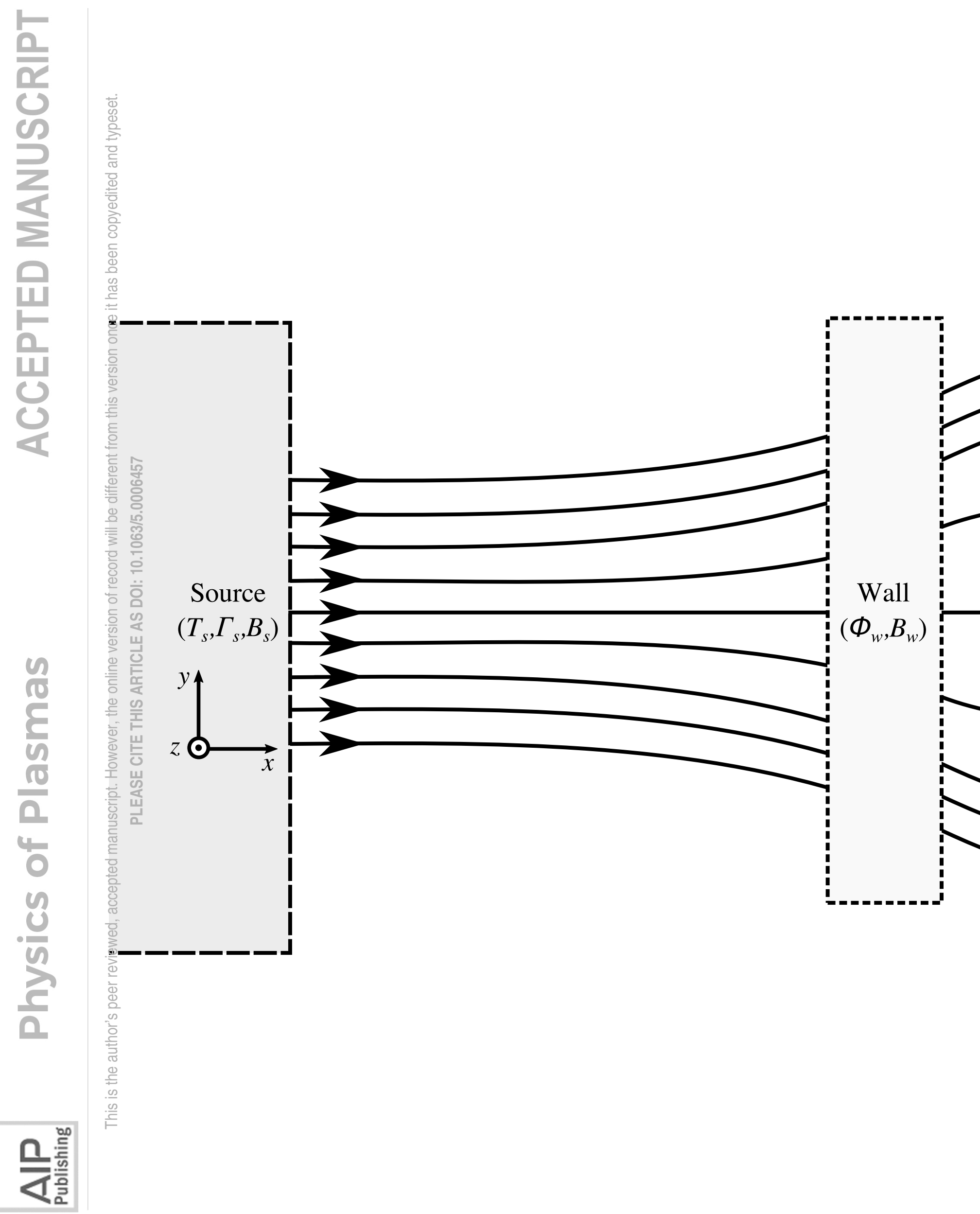



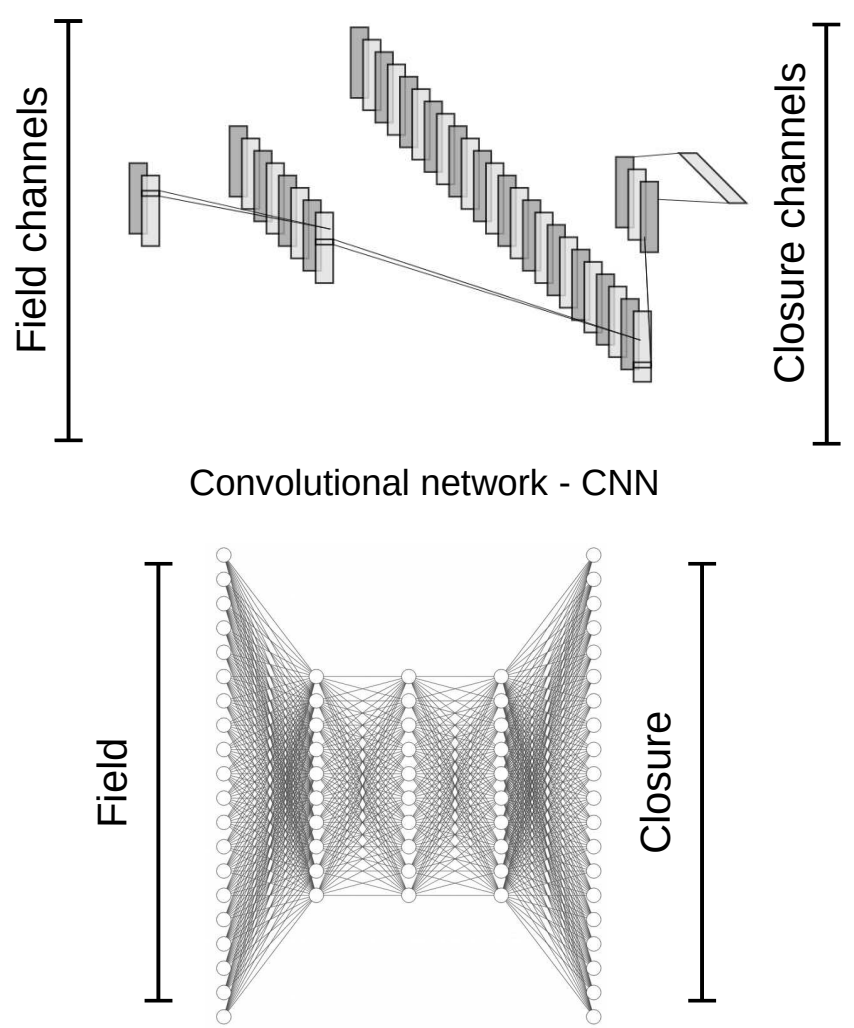

Fully connected network -FNN

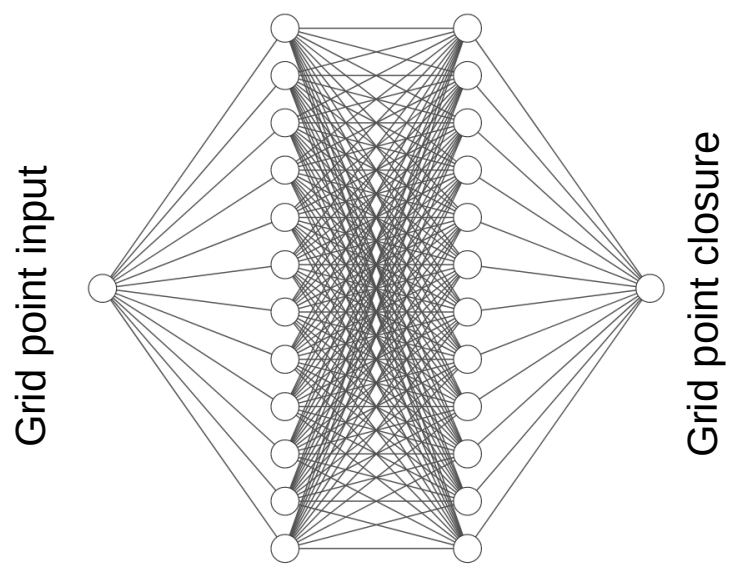

Locally connected network - LNN 


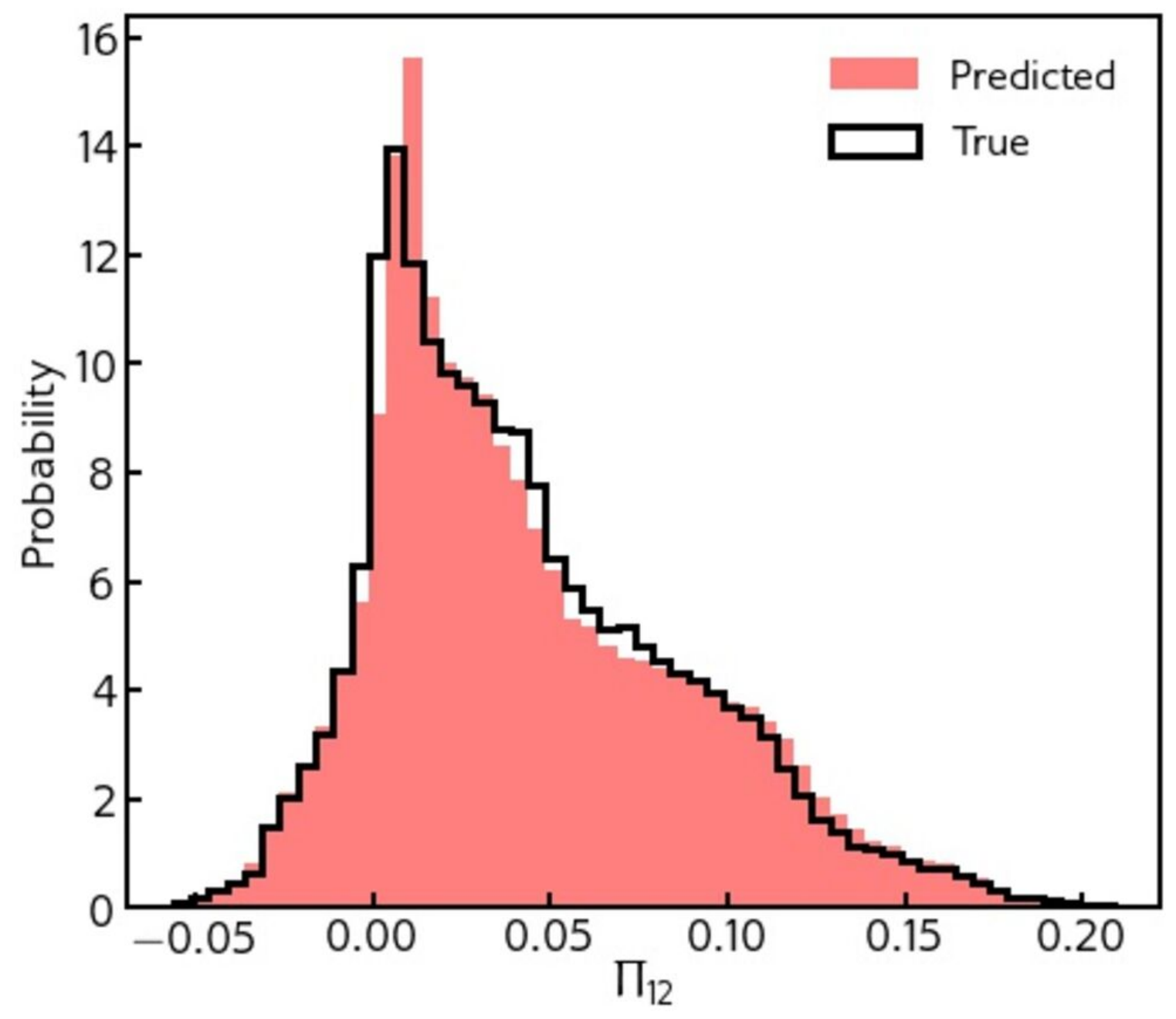

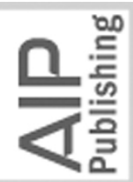




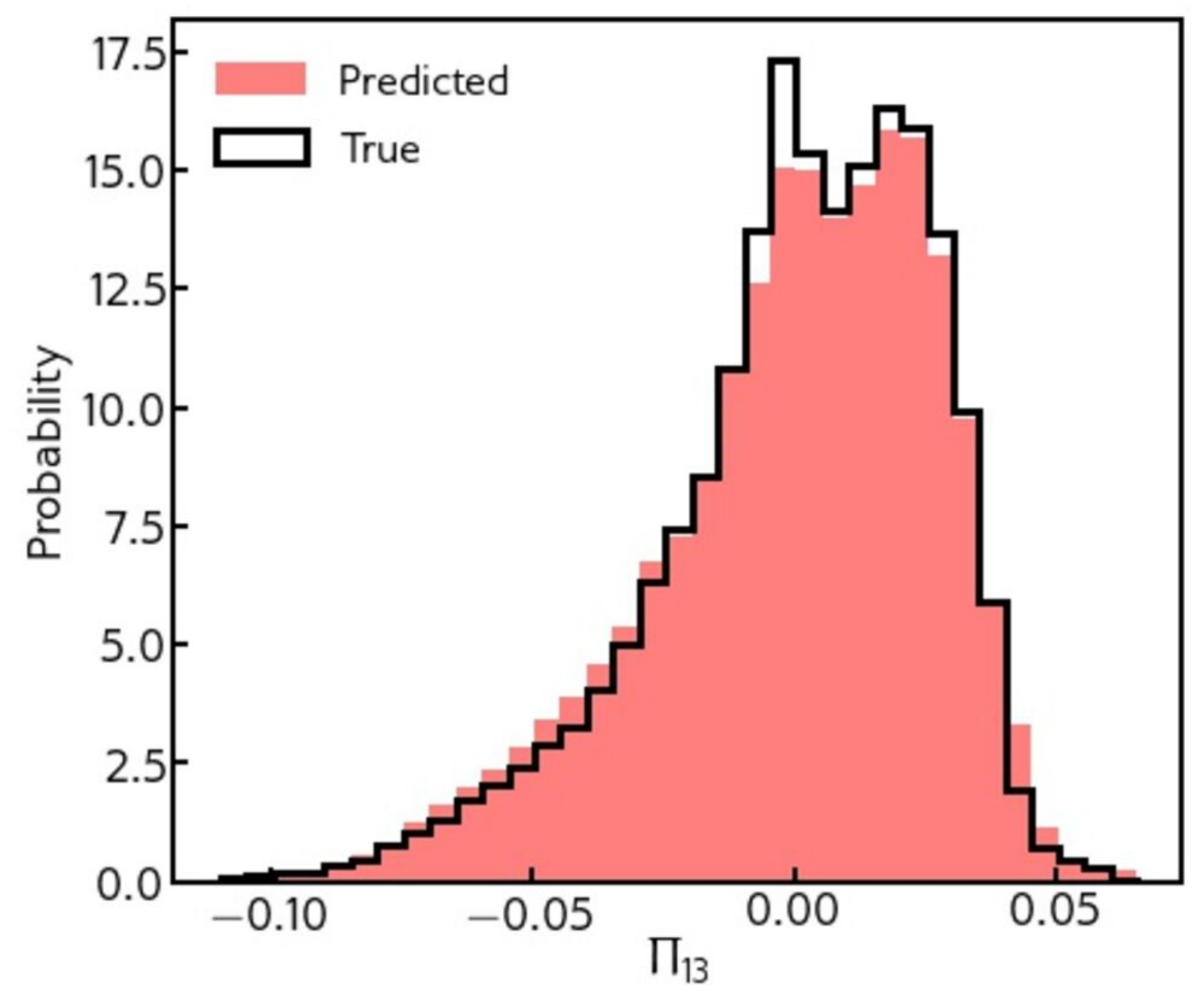

兄耪言 


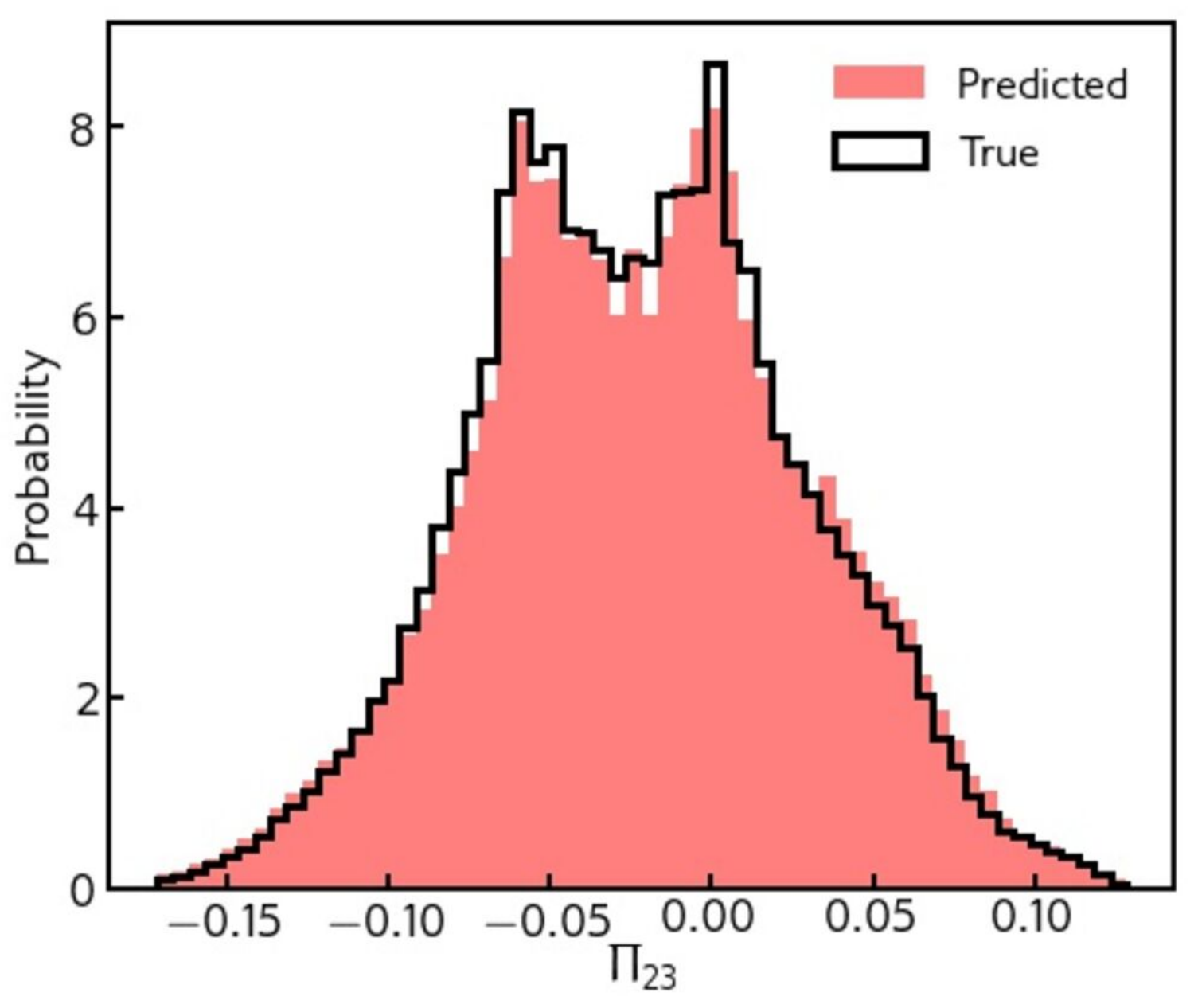

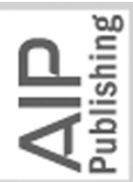




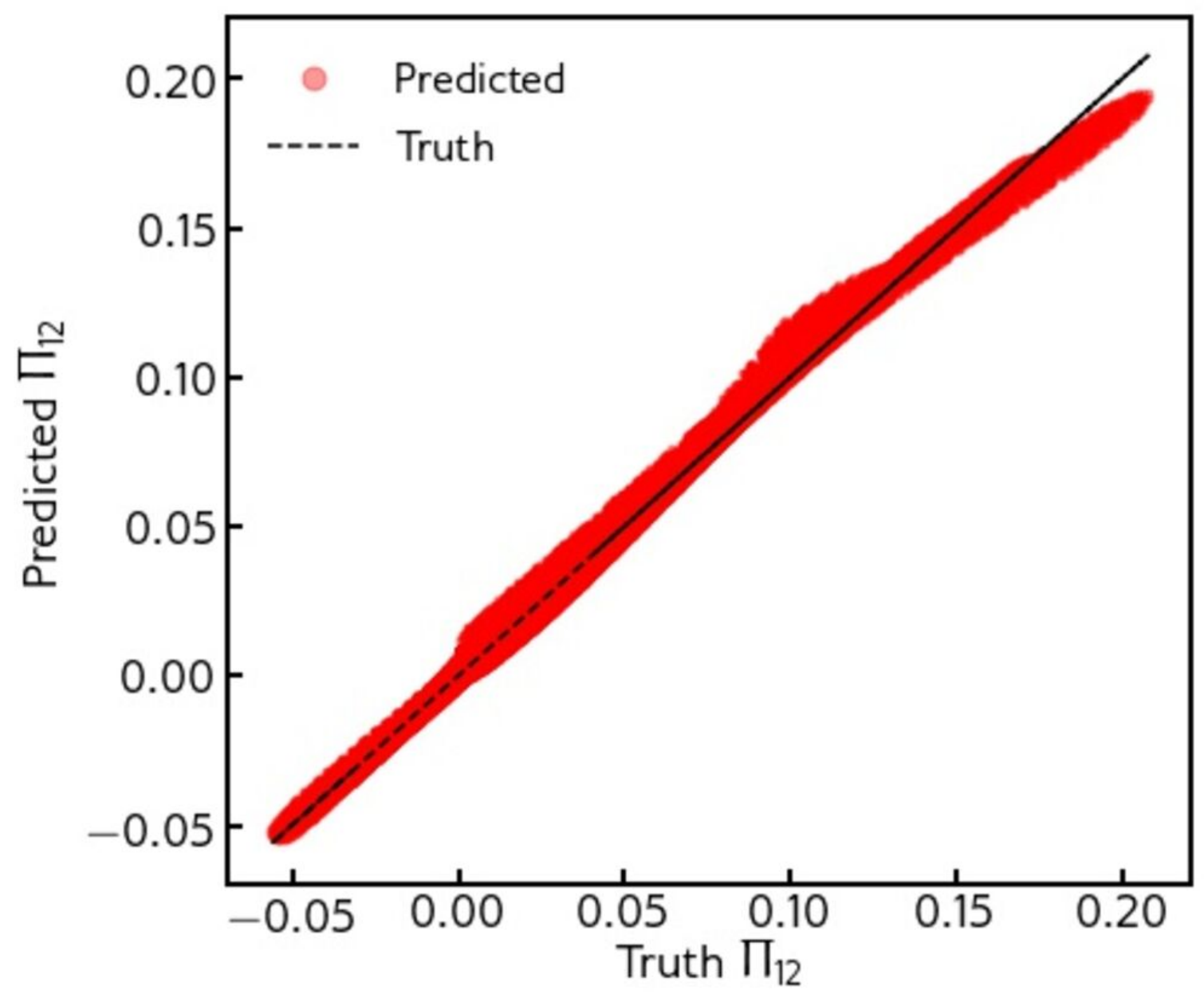

兄耪言 


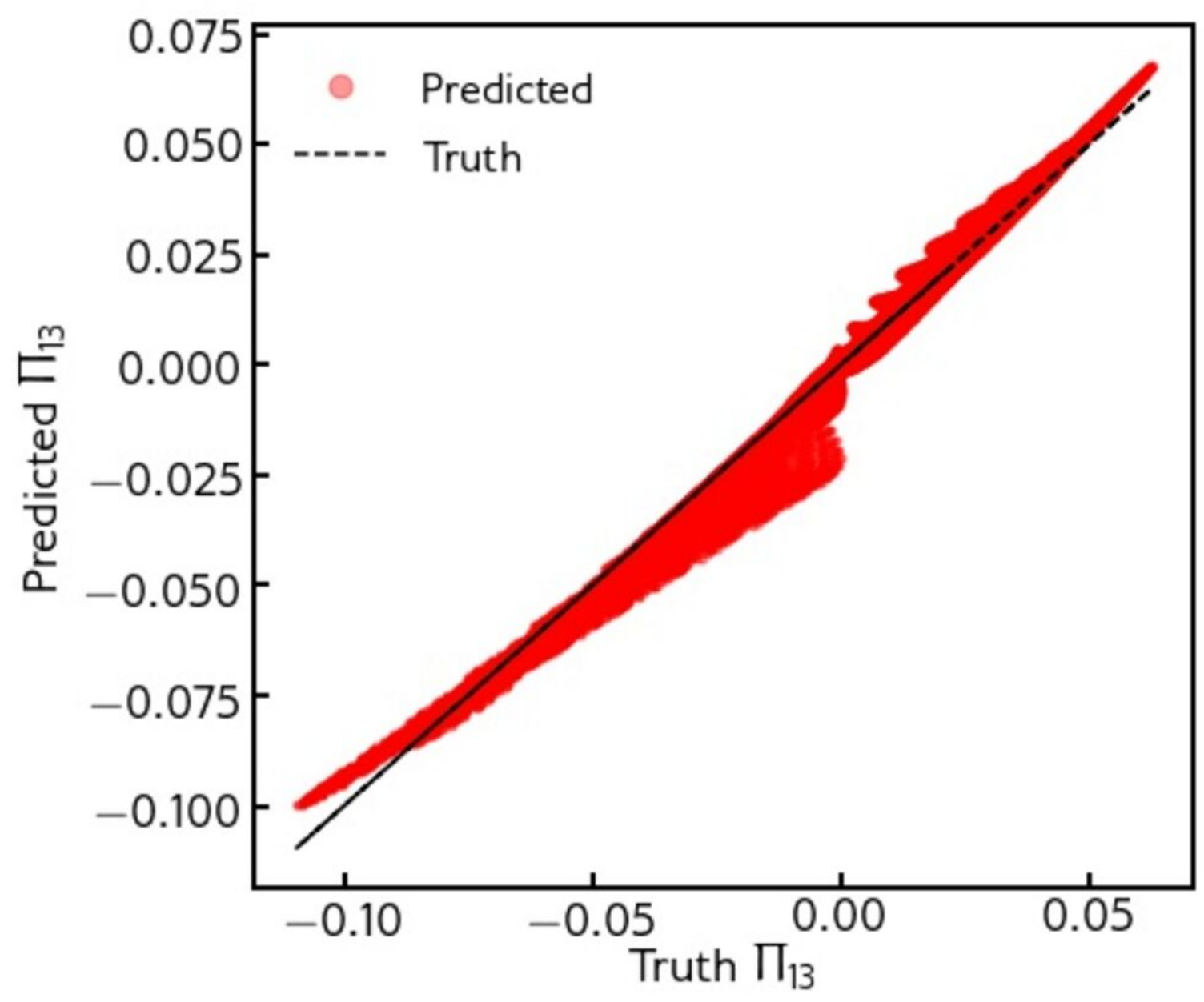

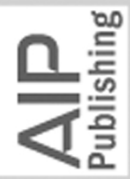




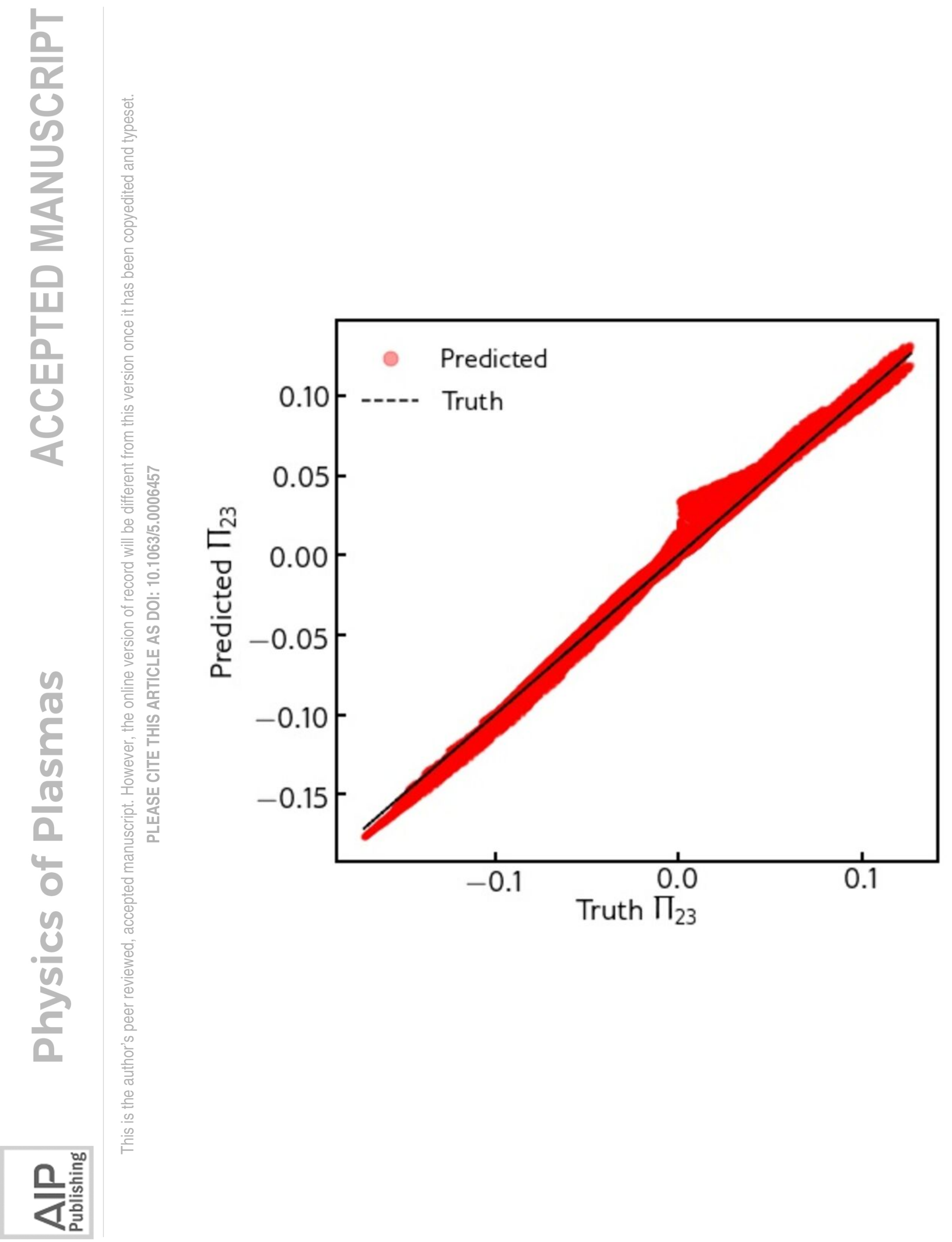




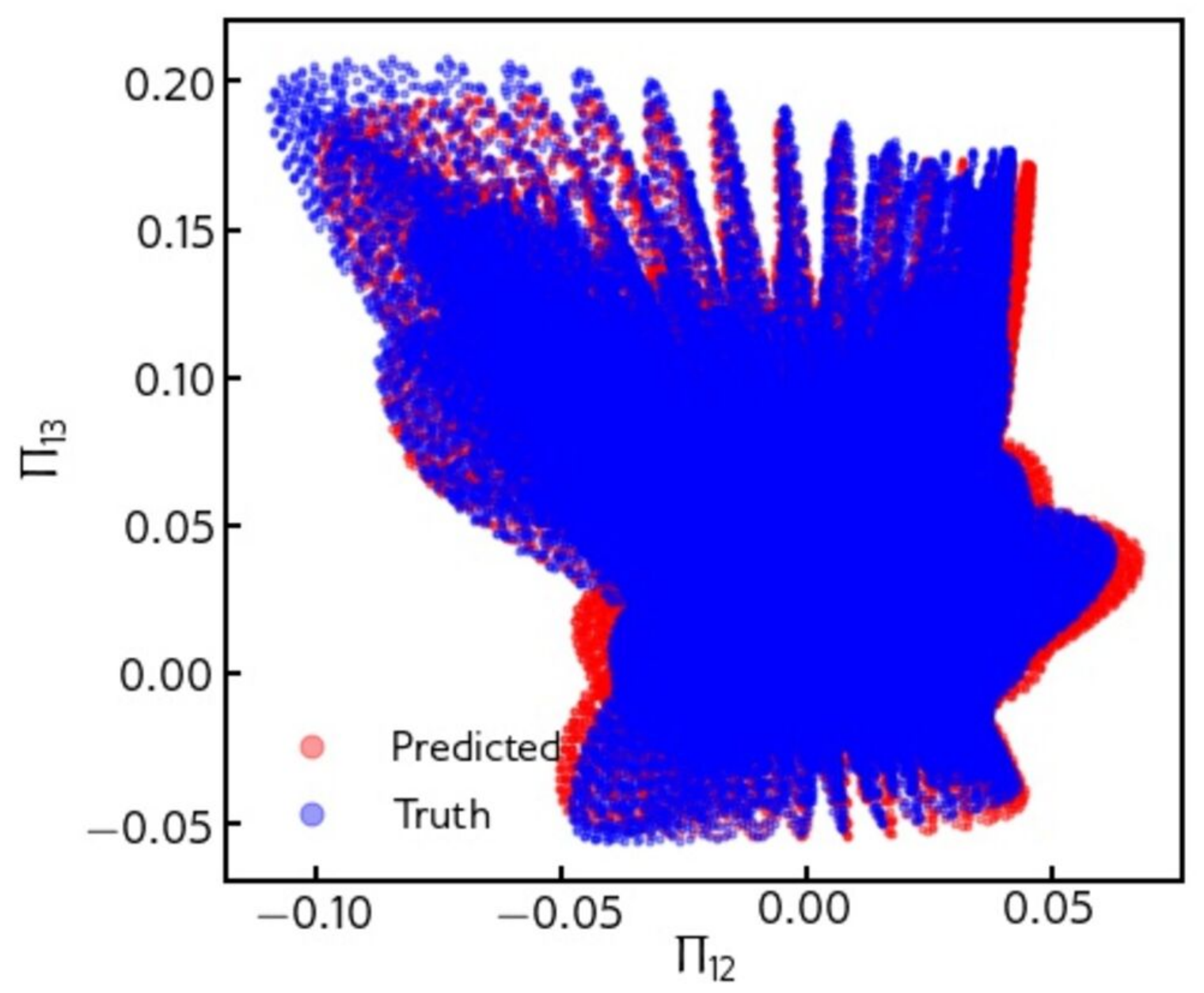

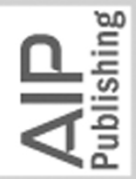




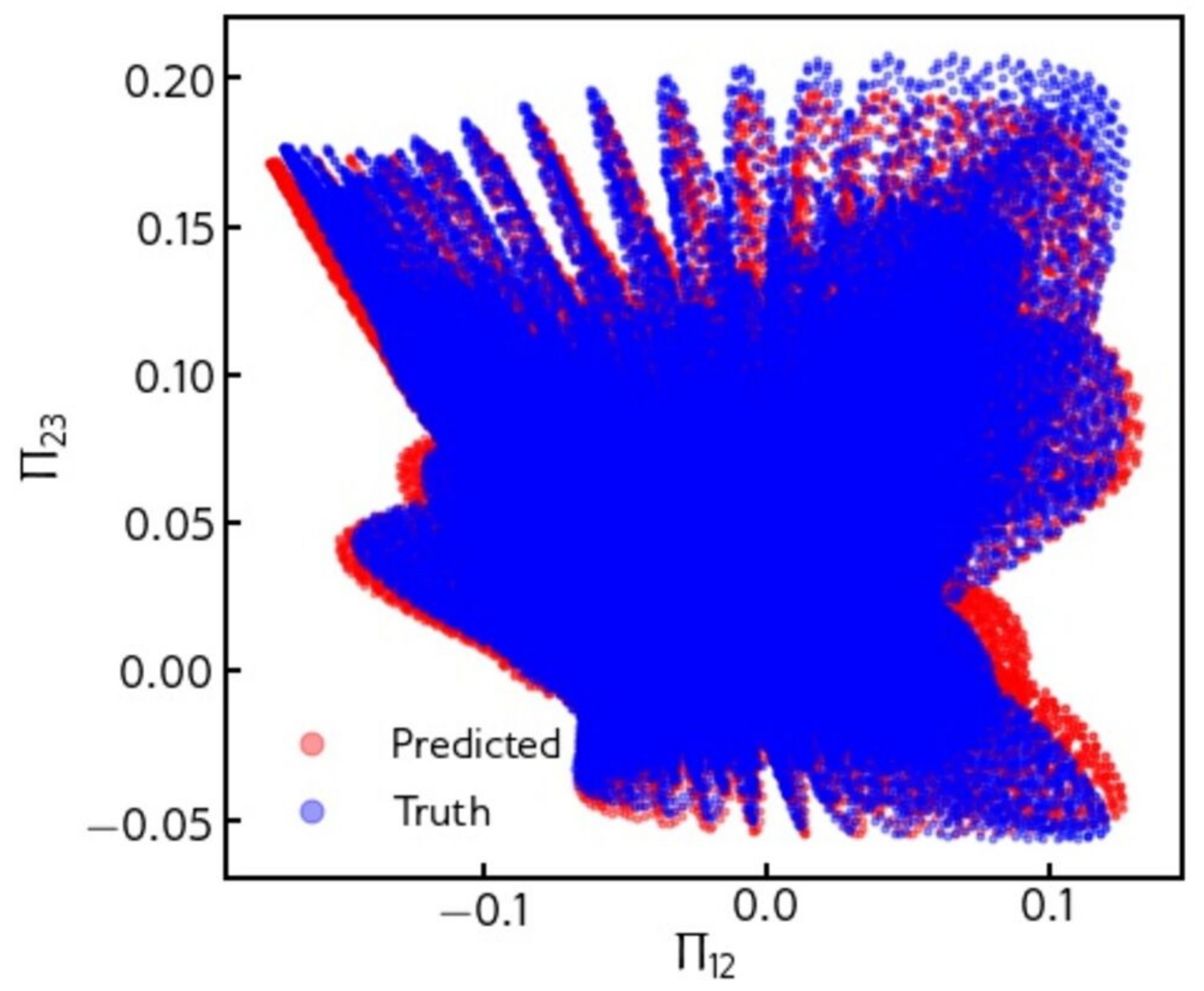

兄耪言 


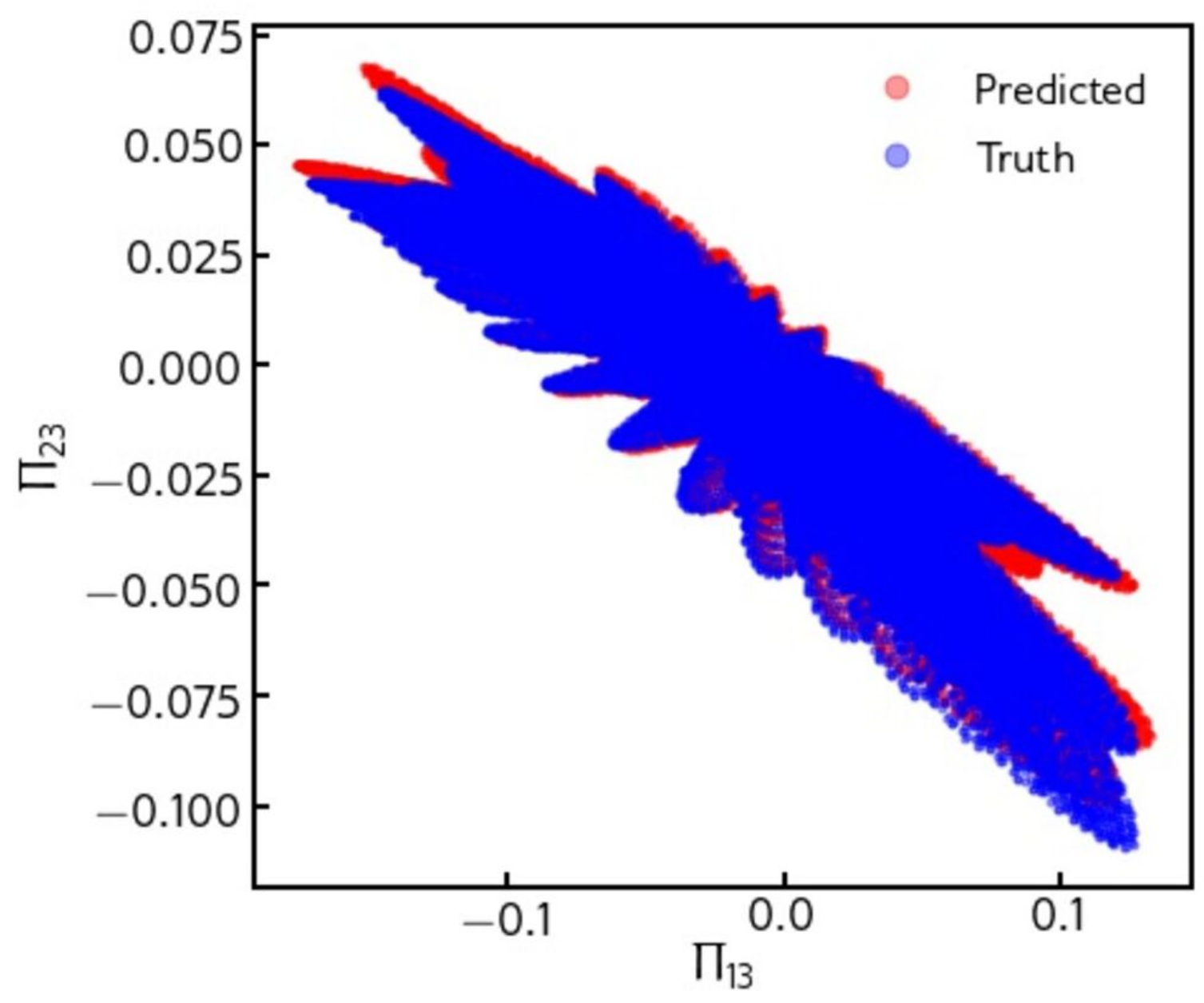

兄耪言 


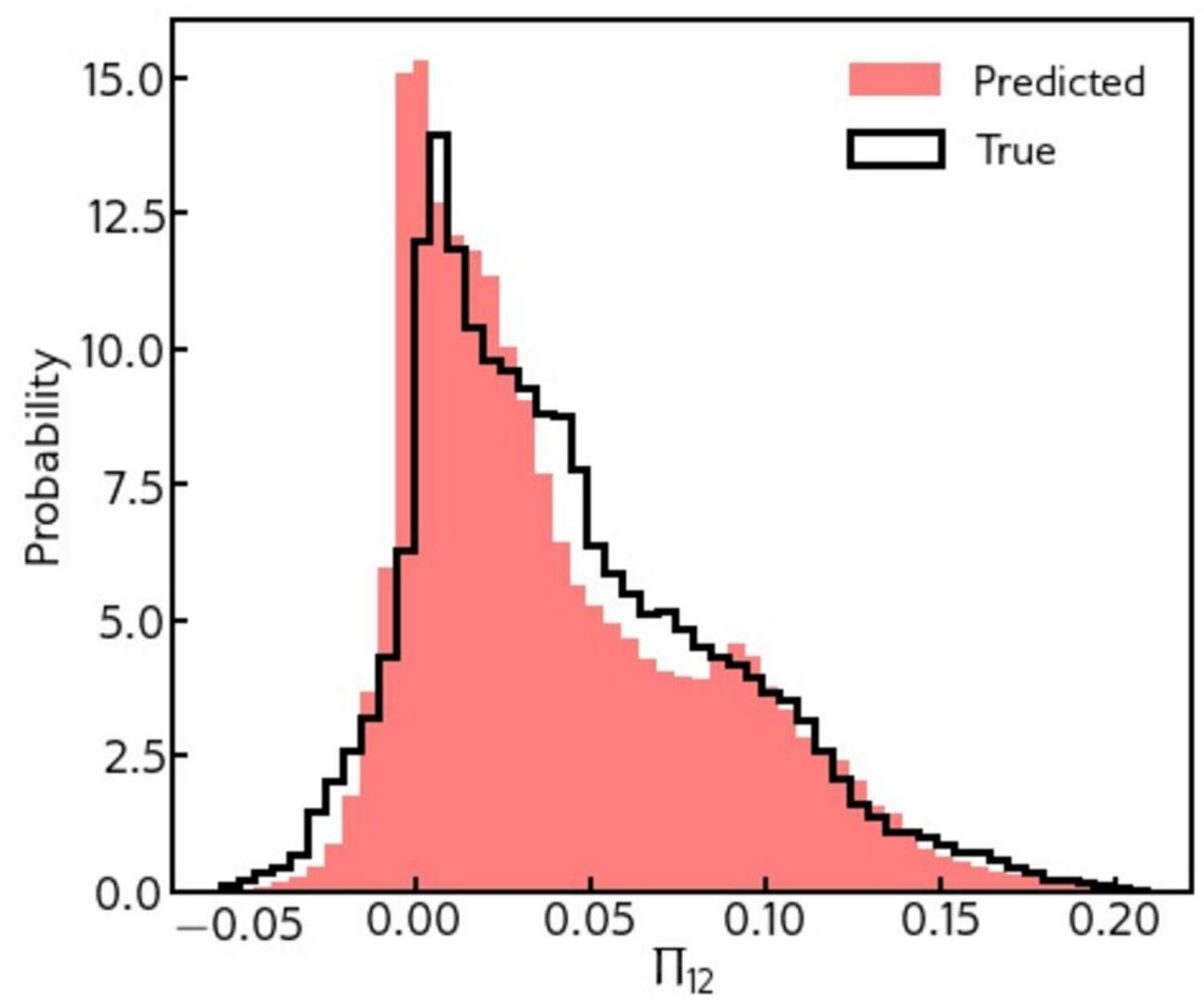

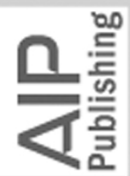




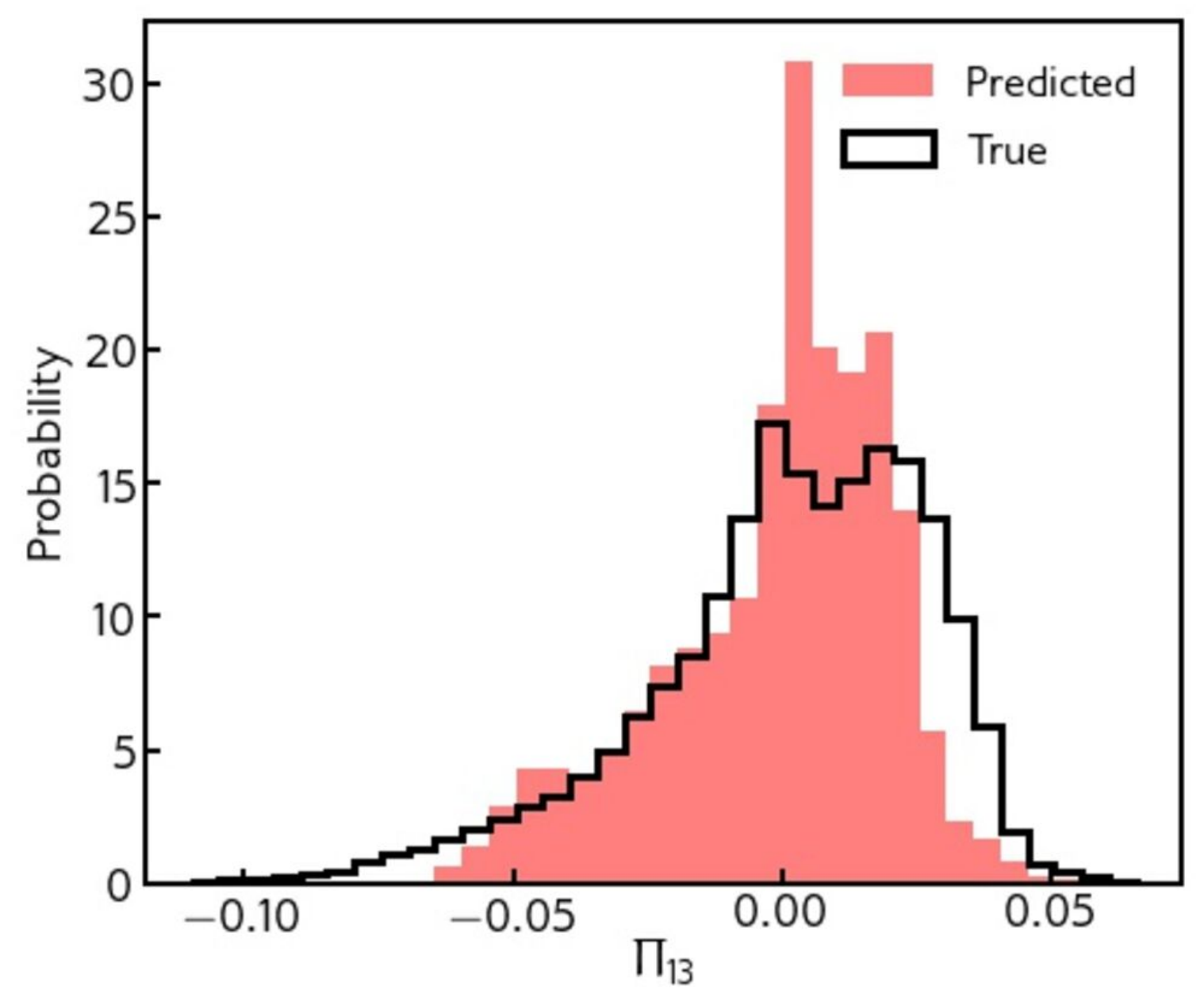

은 


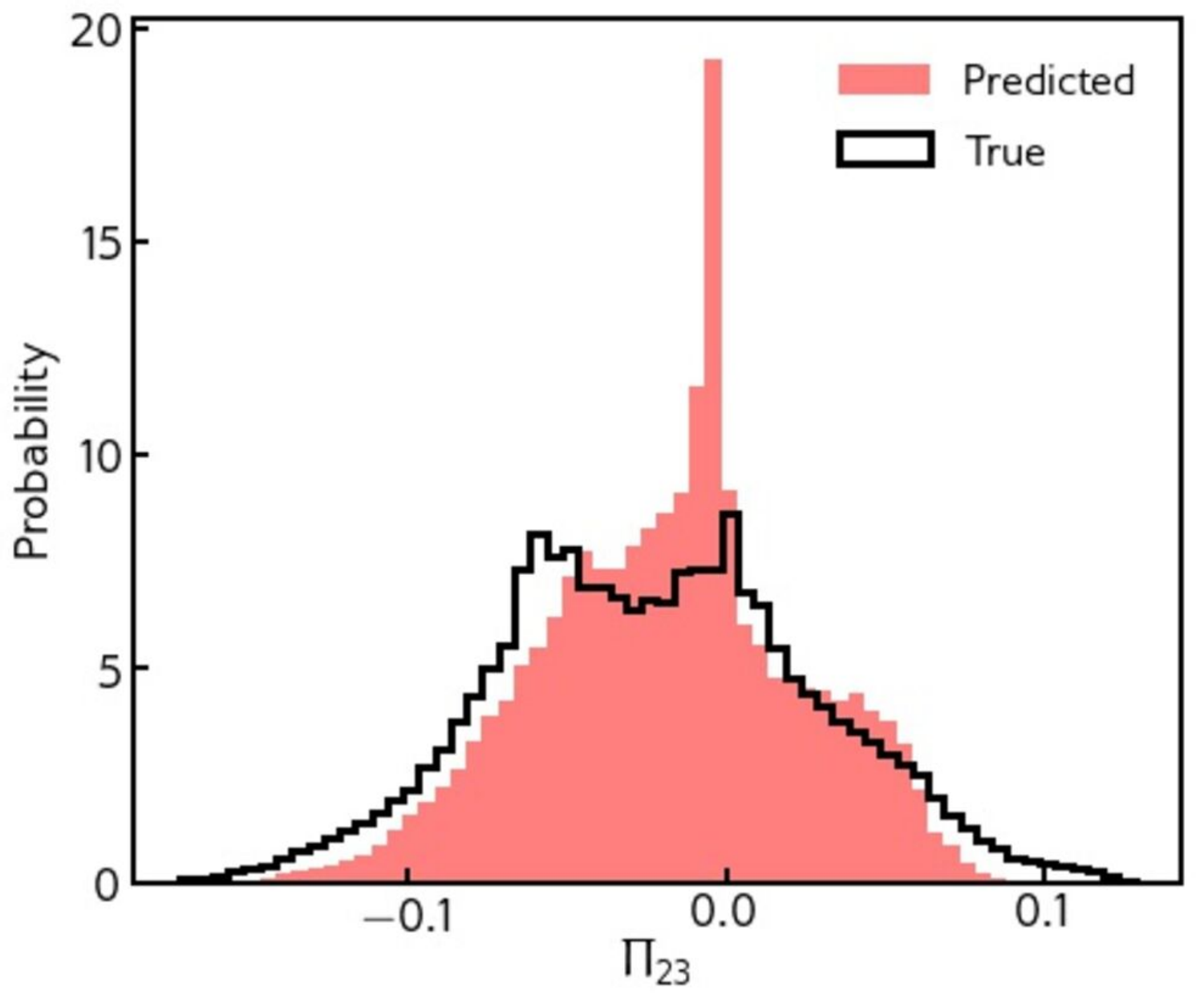

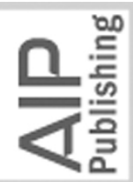




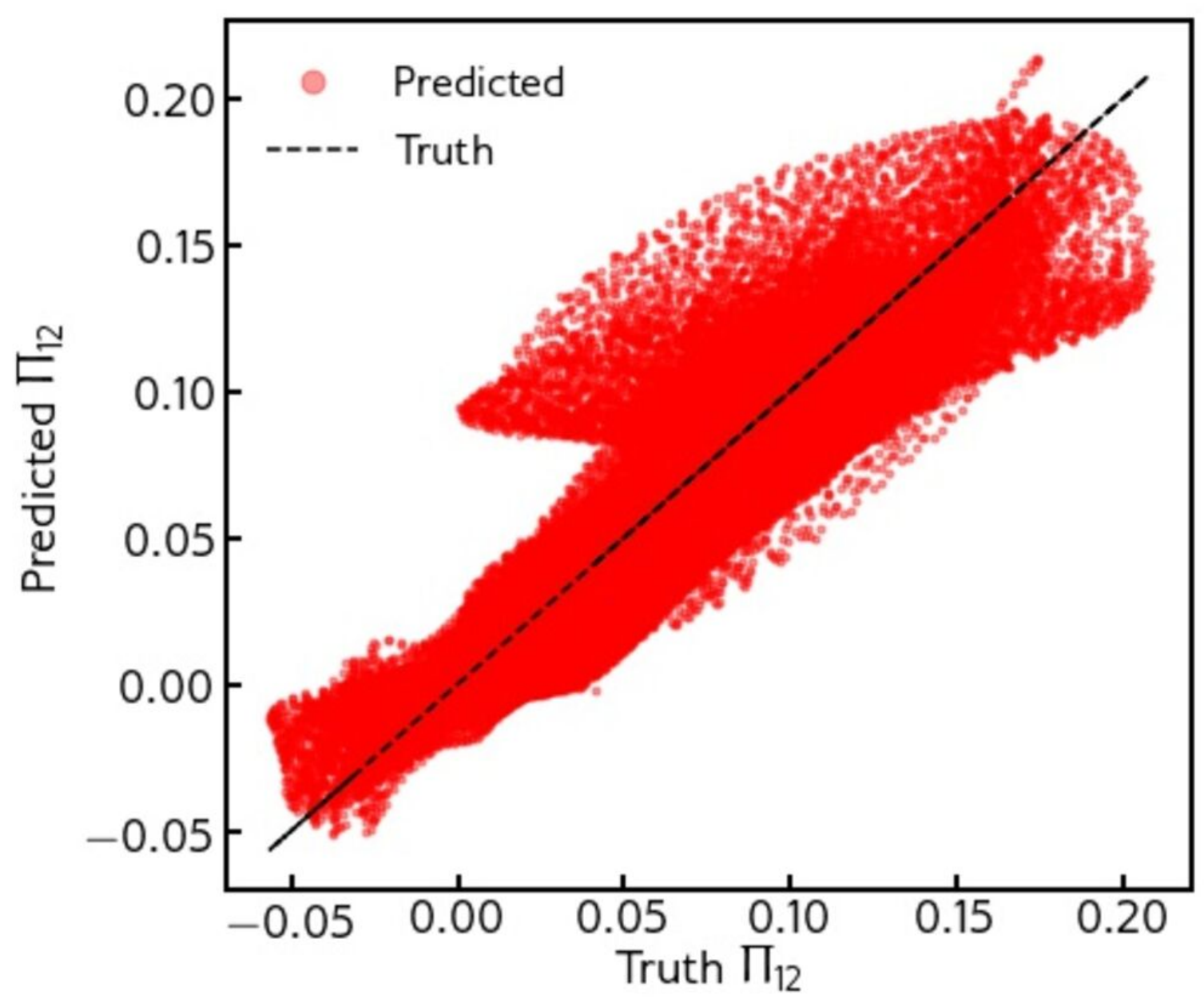

兄耪言 


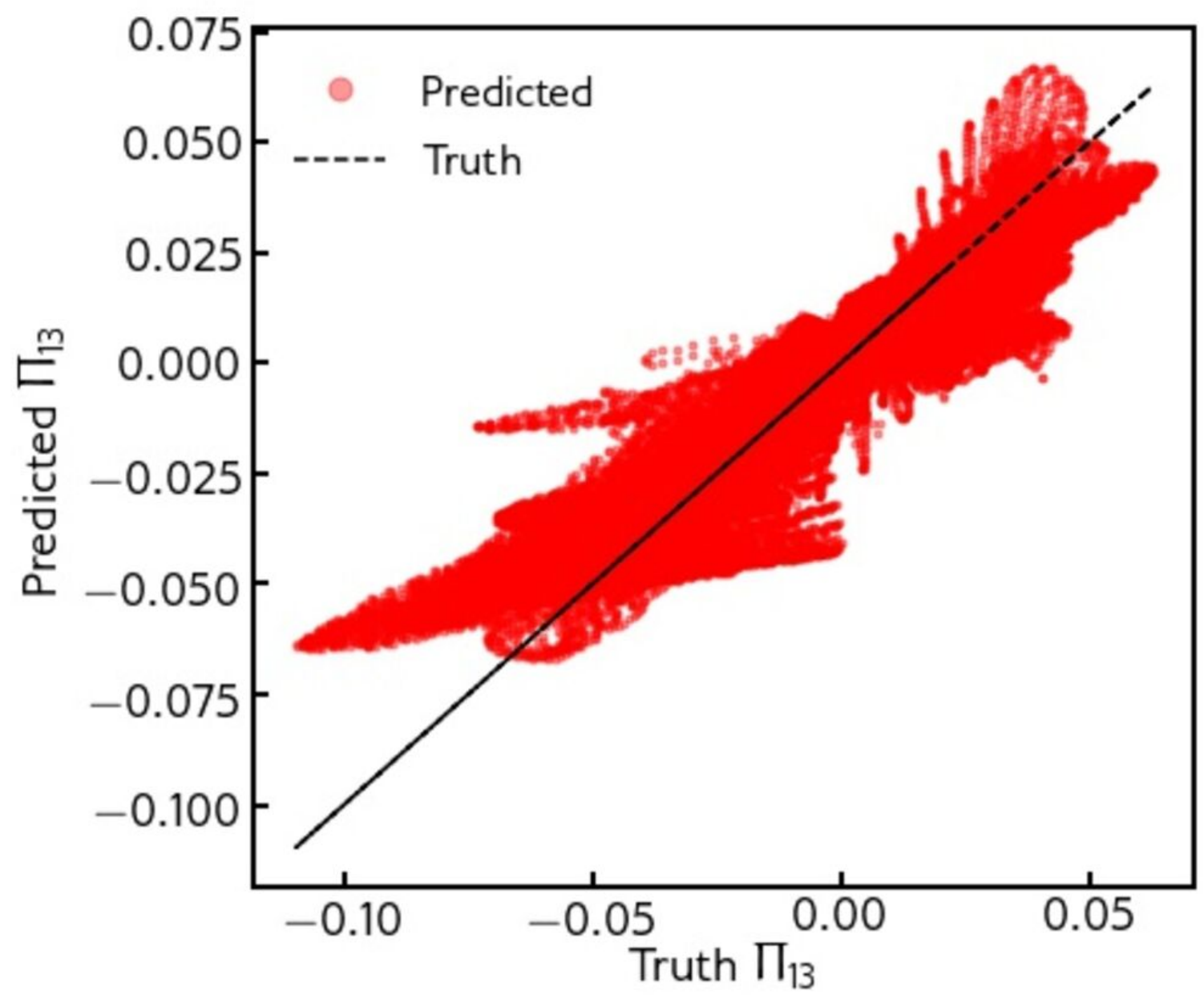

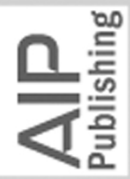




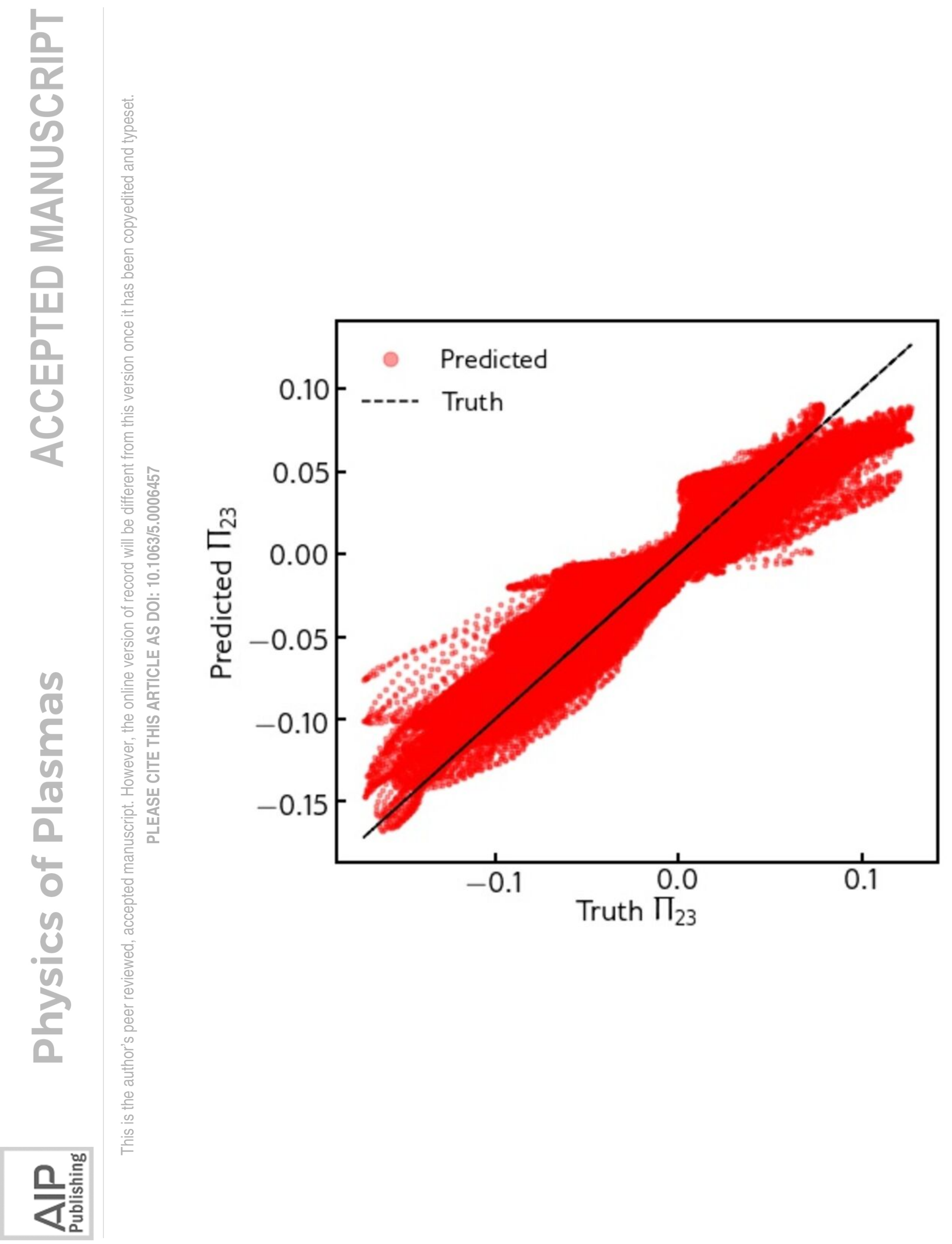






兄耪言 


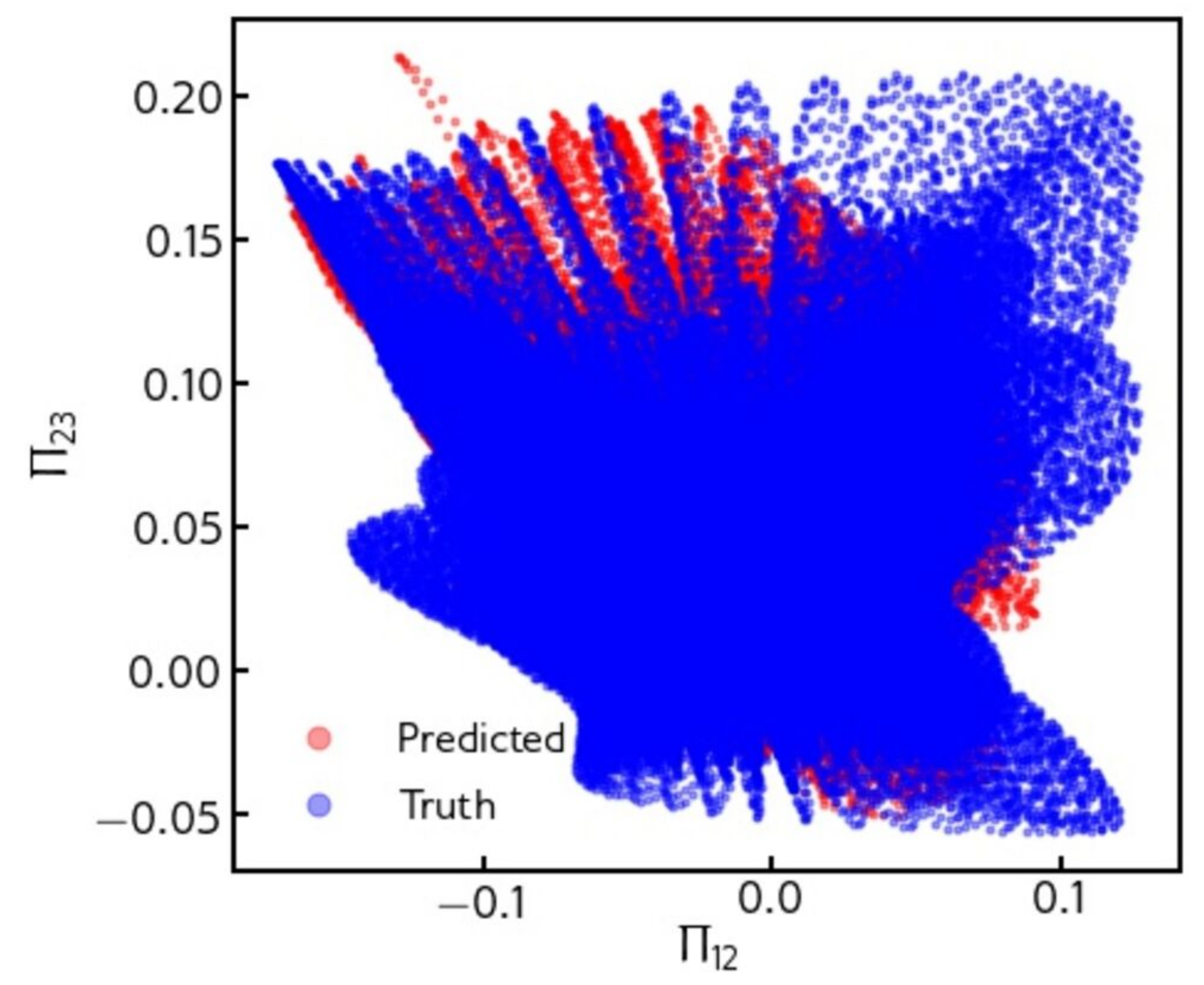

兄耪言 


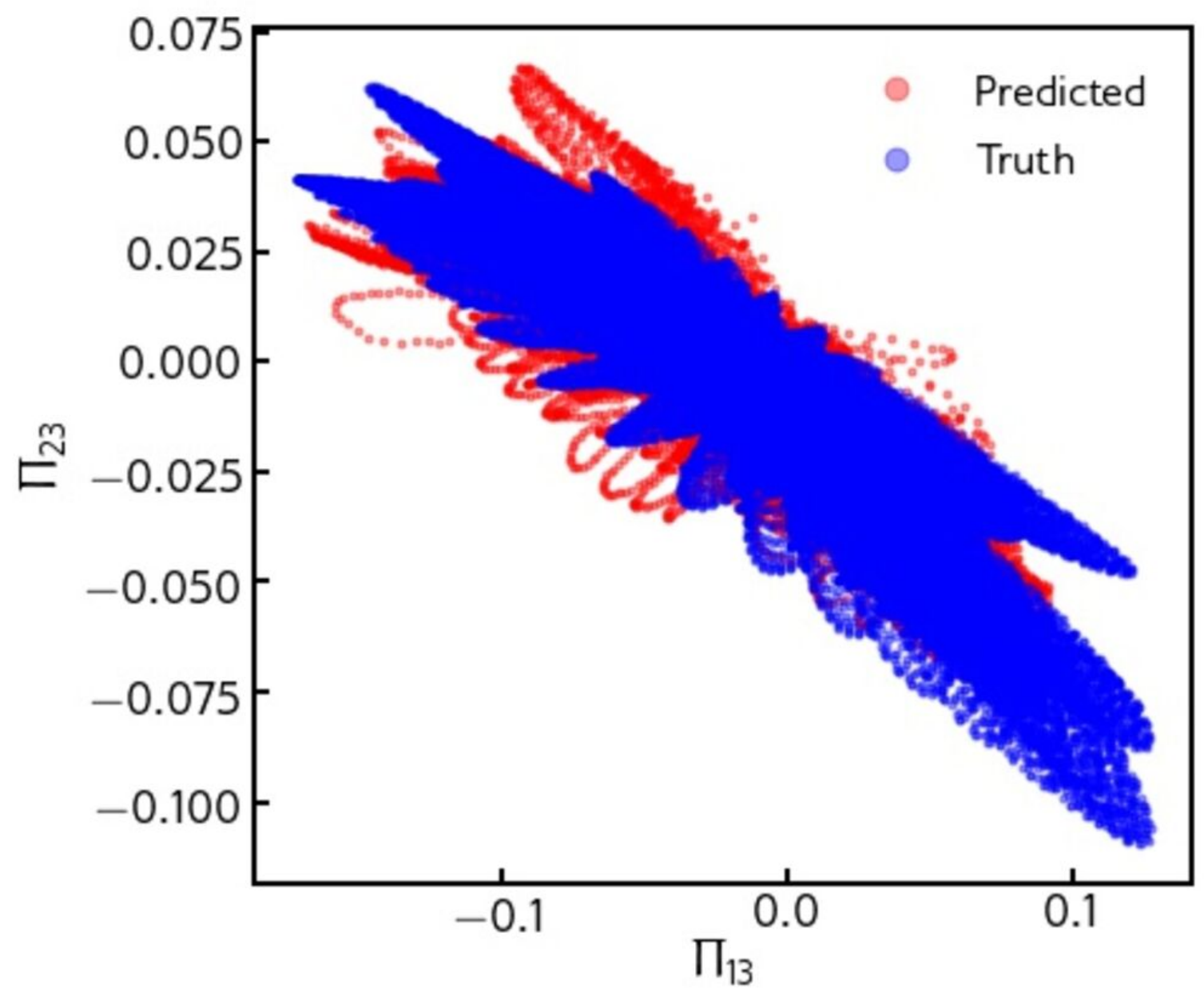

兄耪言 


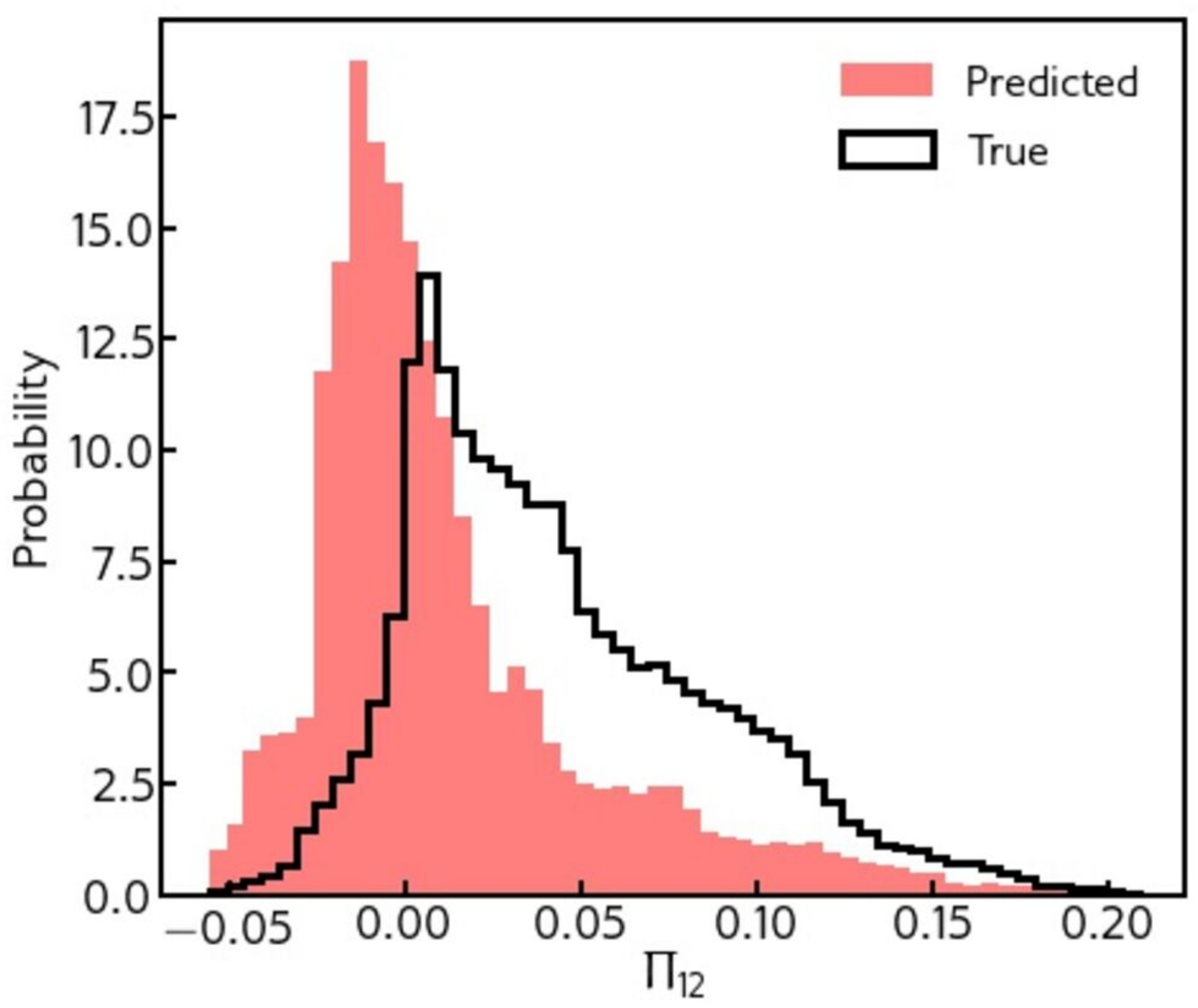

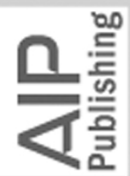




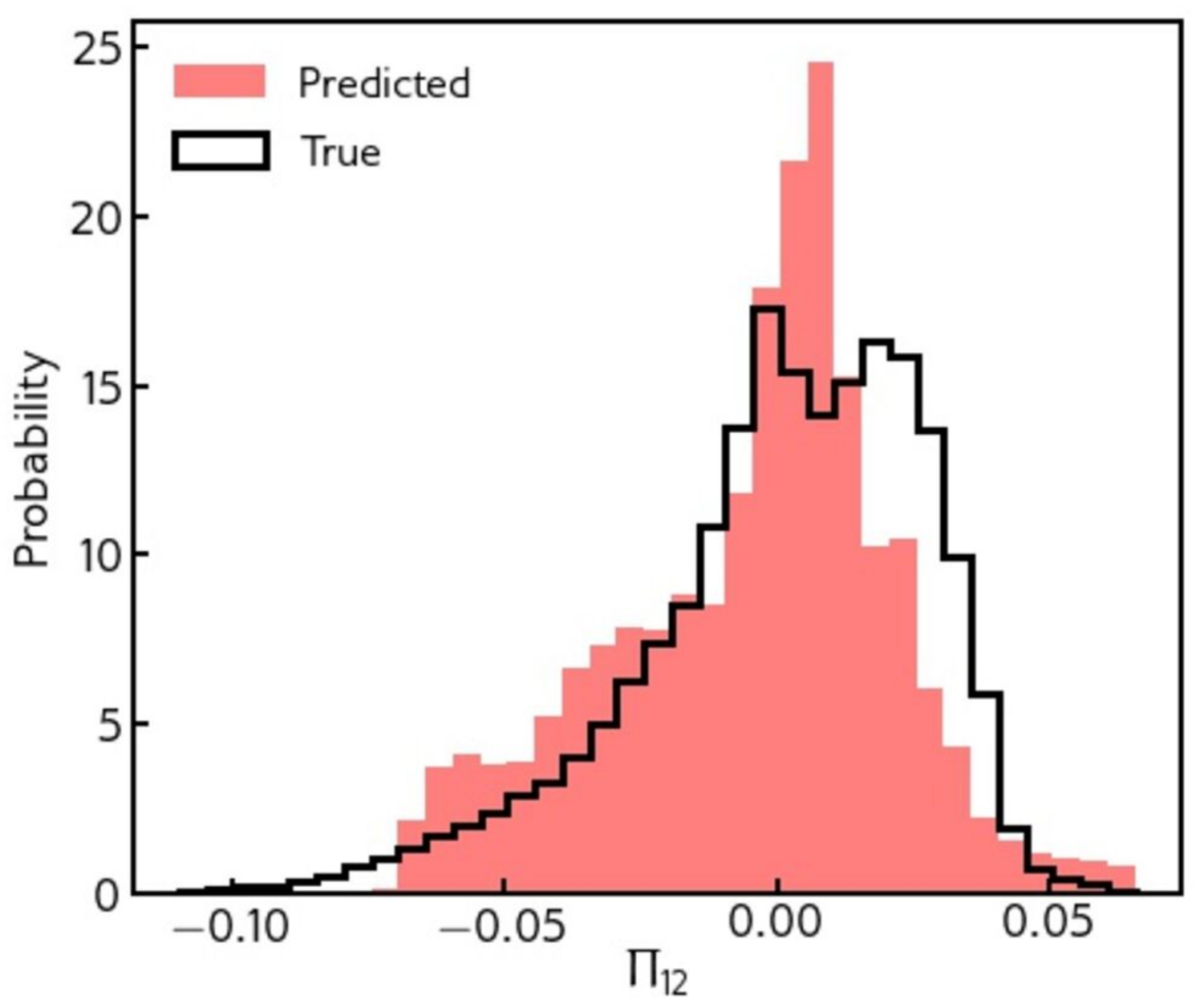

는 


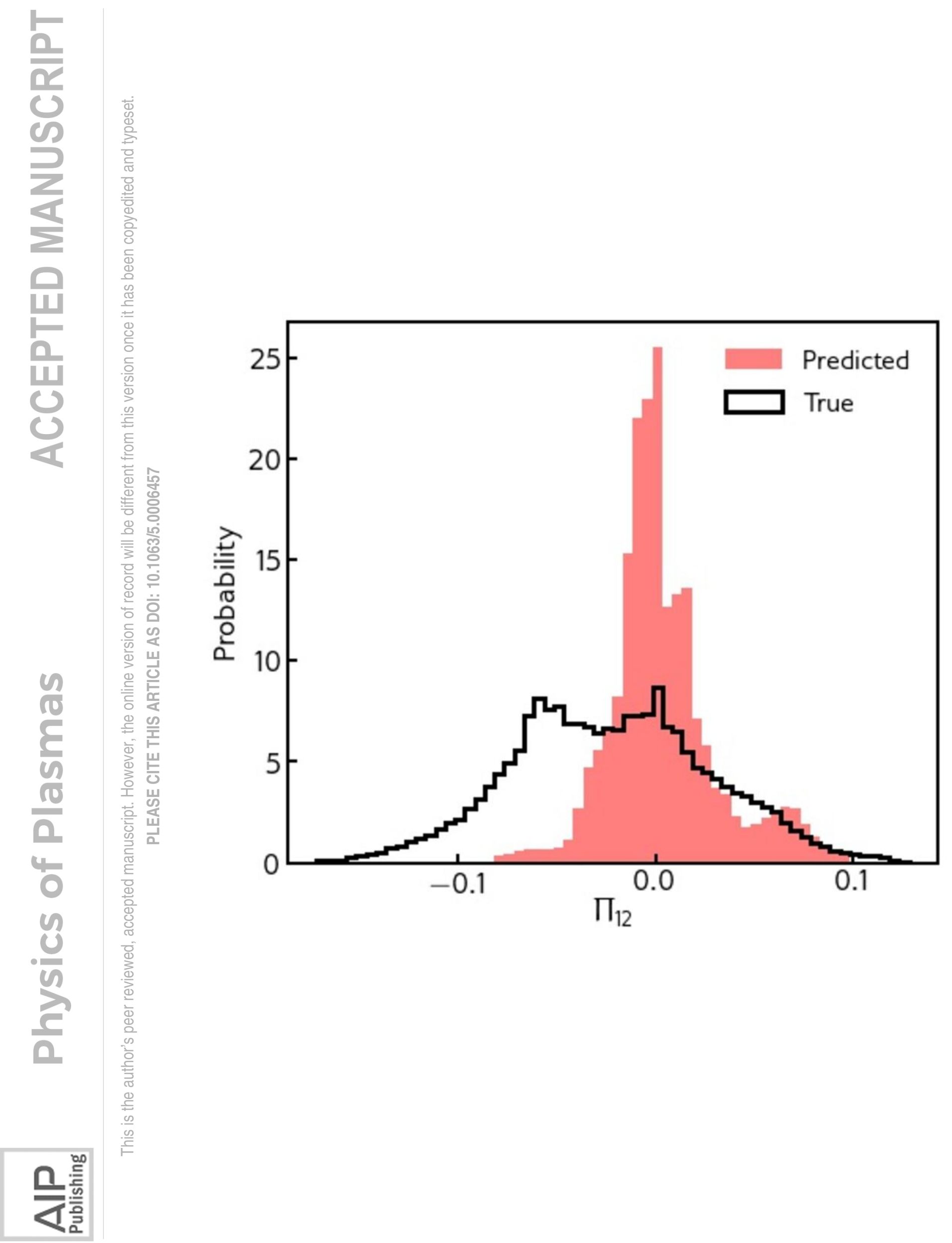




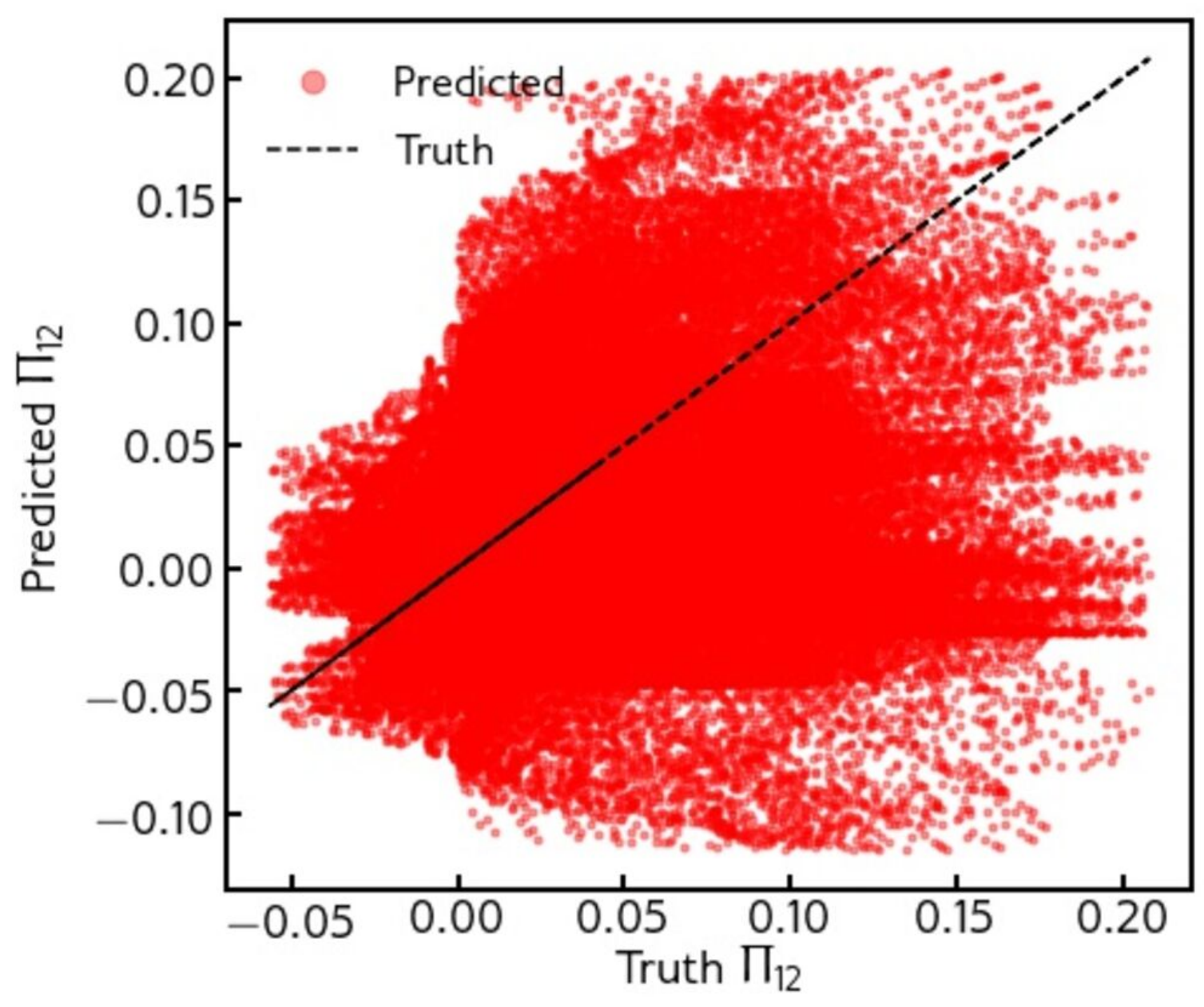

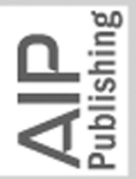




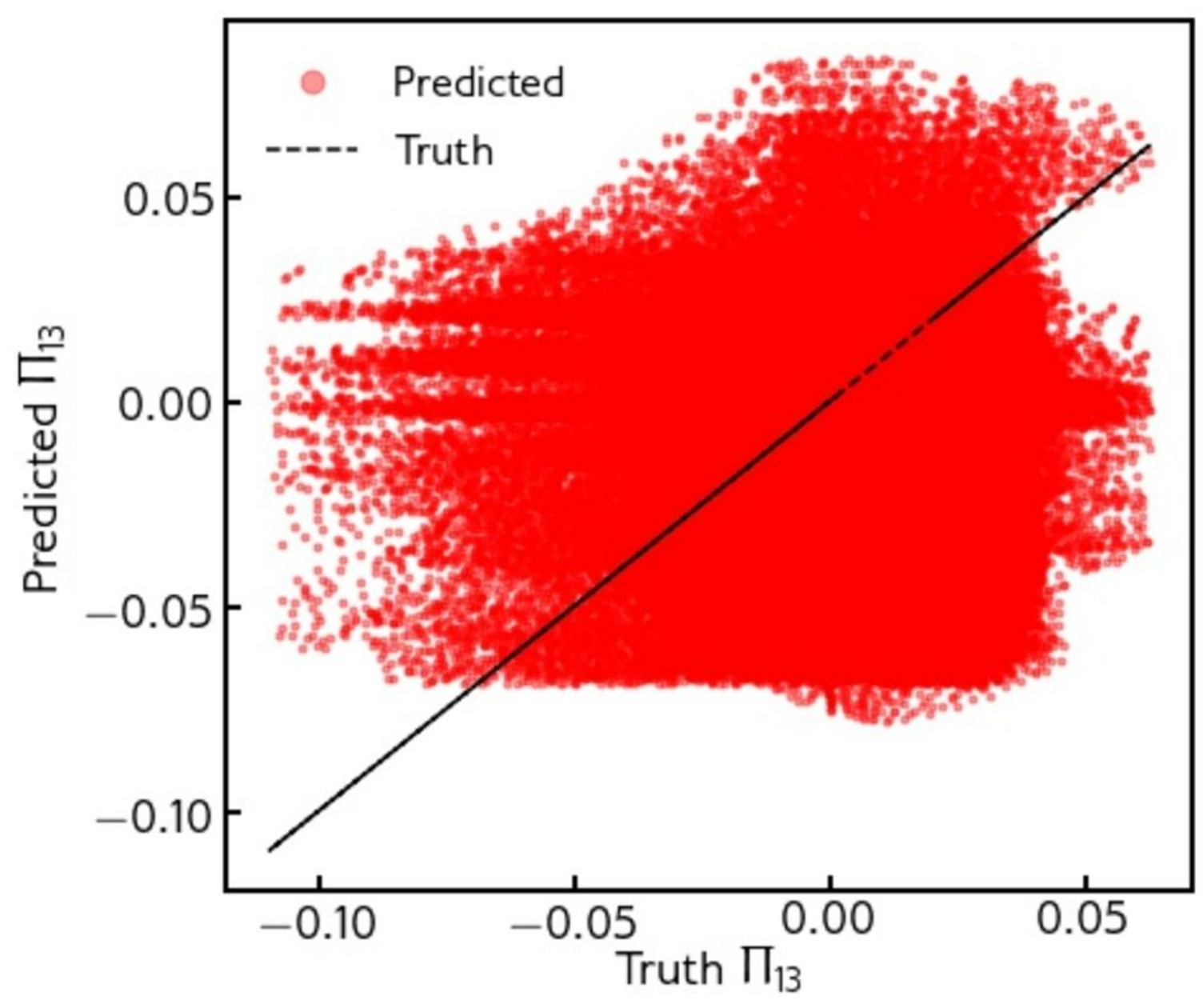

兄耪言 


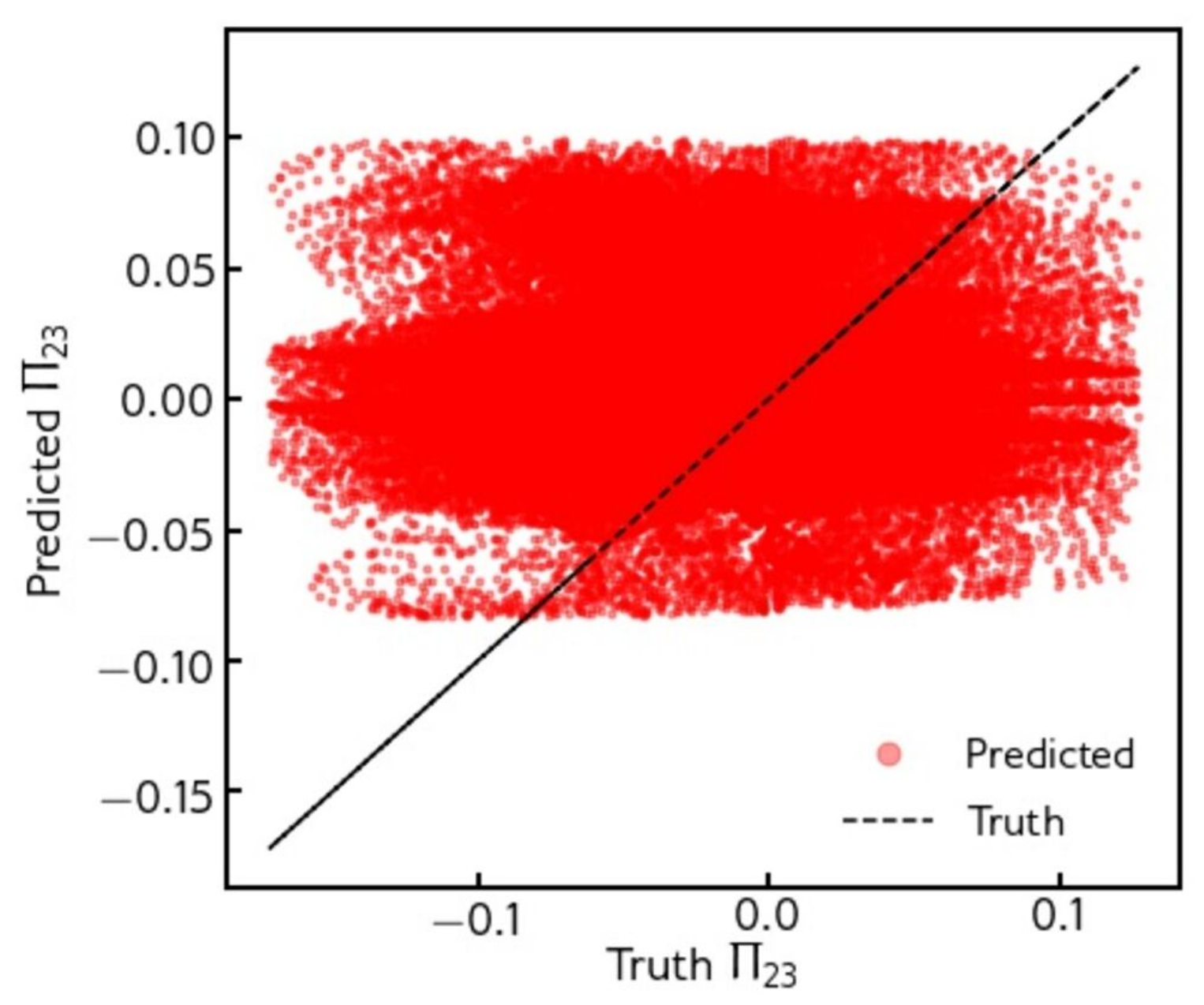

兄耪言 


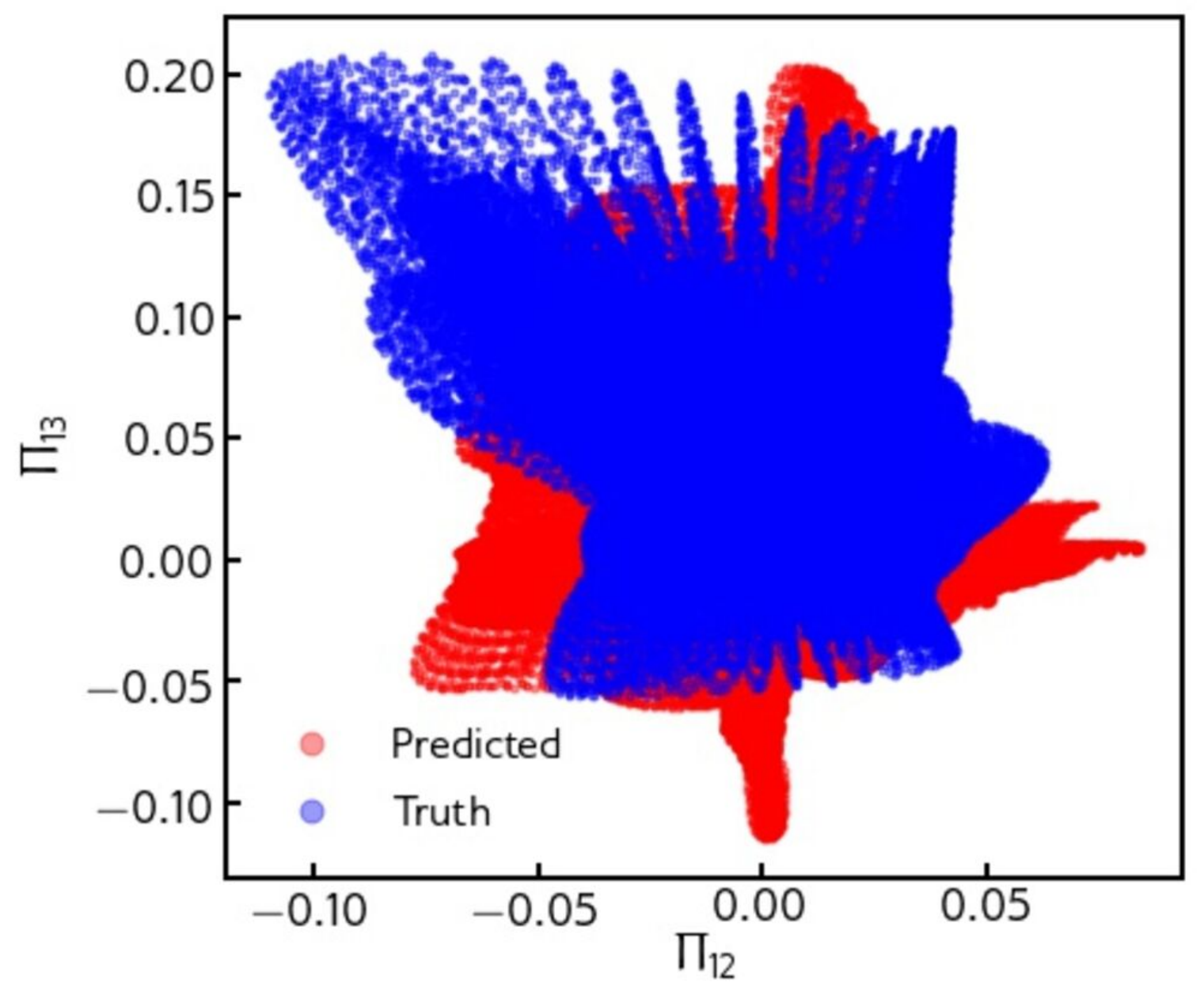

兄耪言 


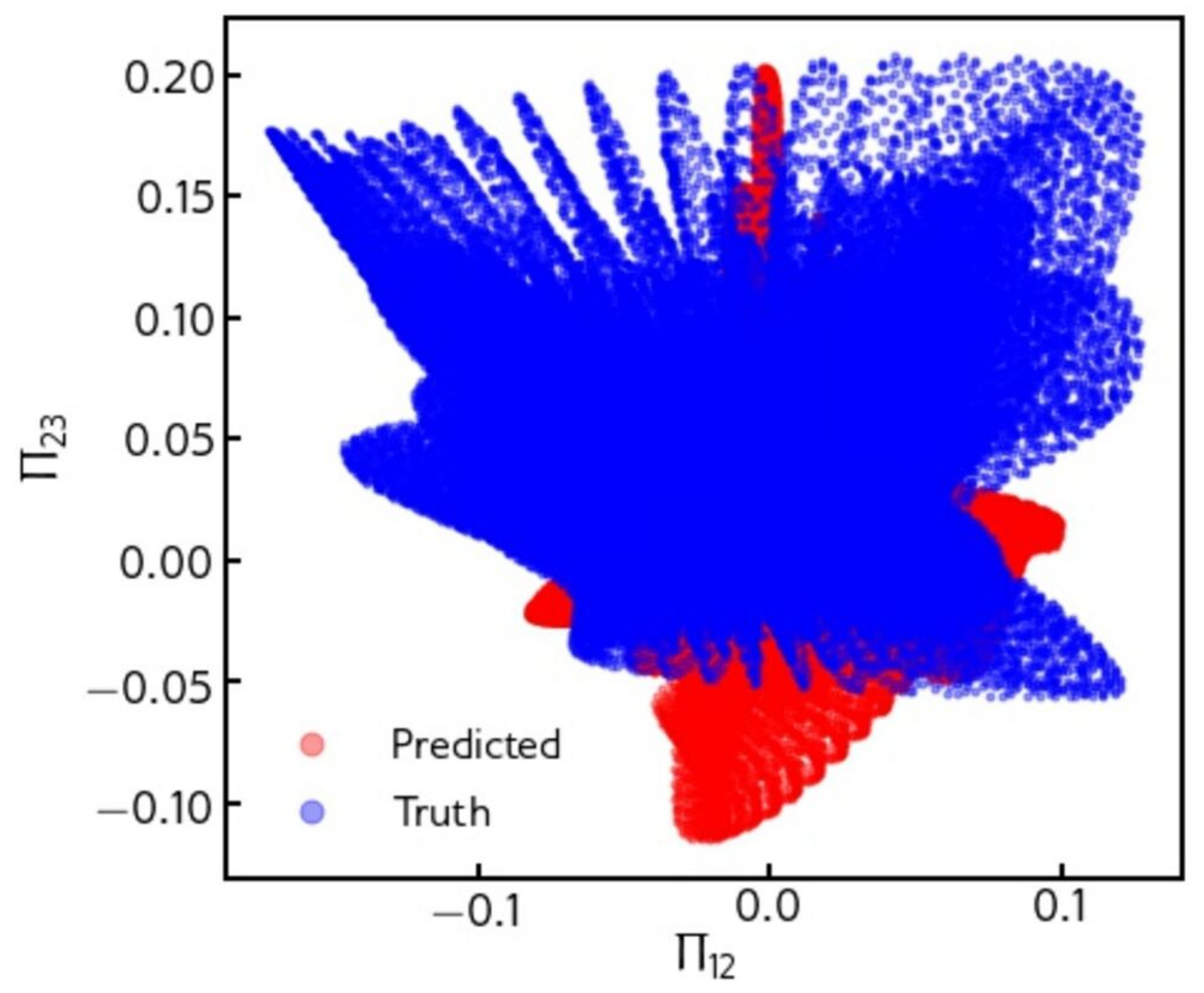

兄耪言 


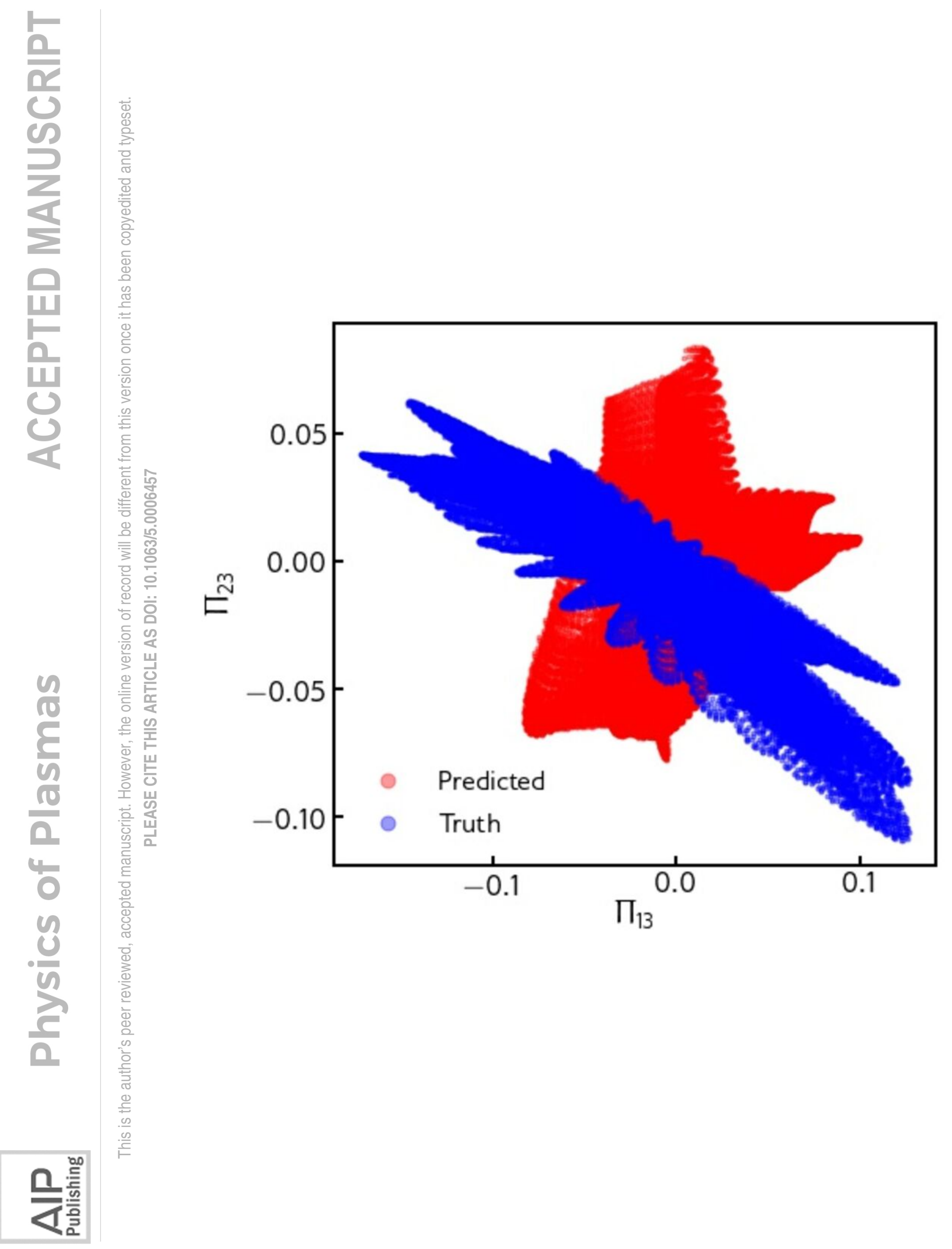




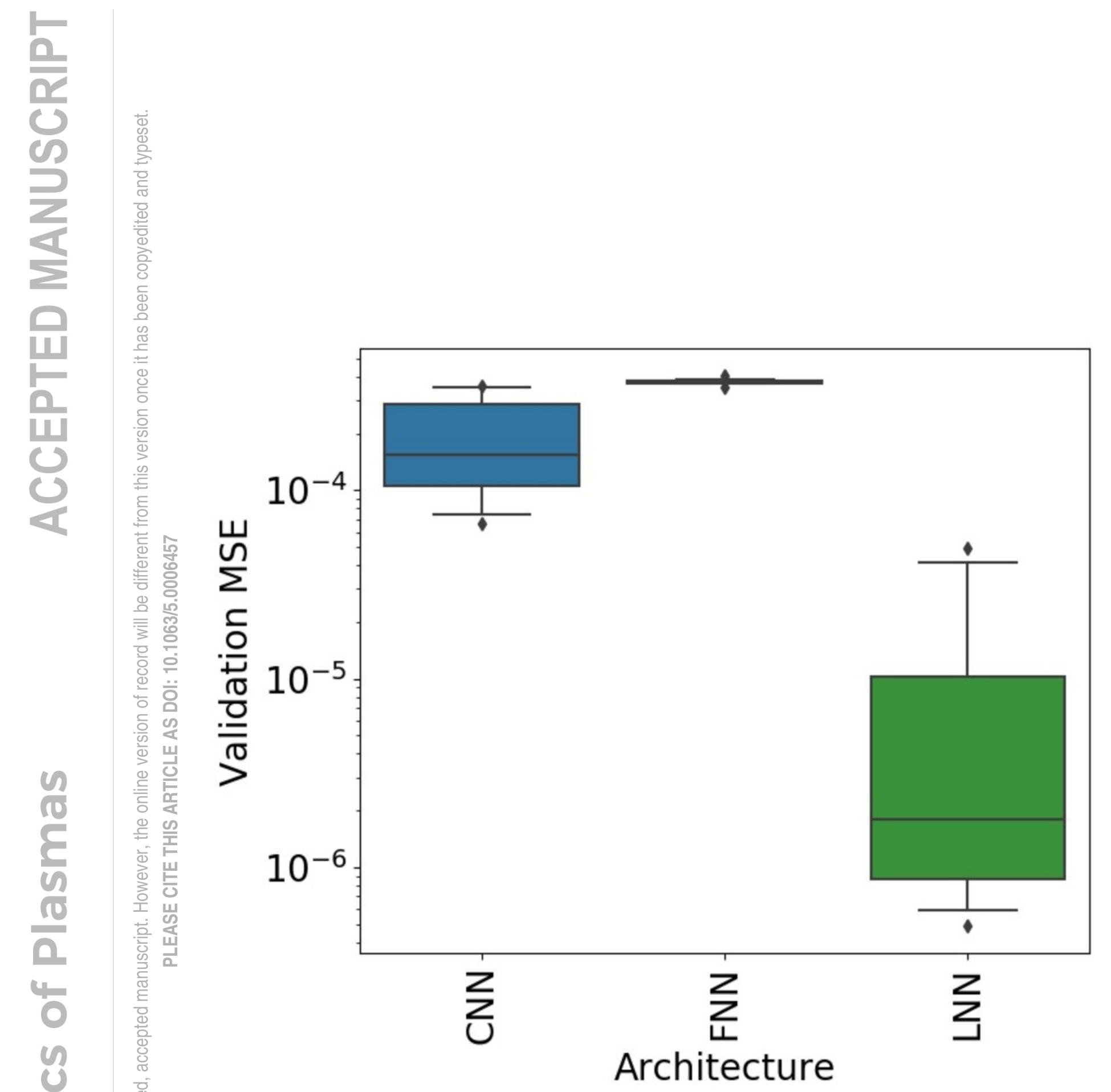

兄旁高 


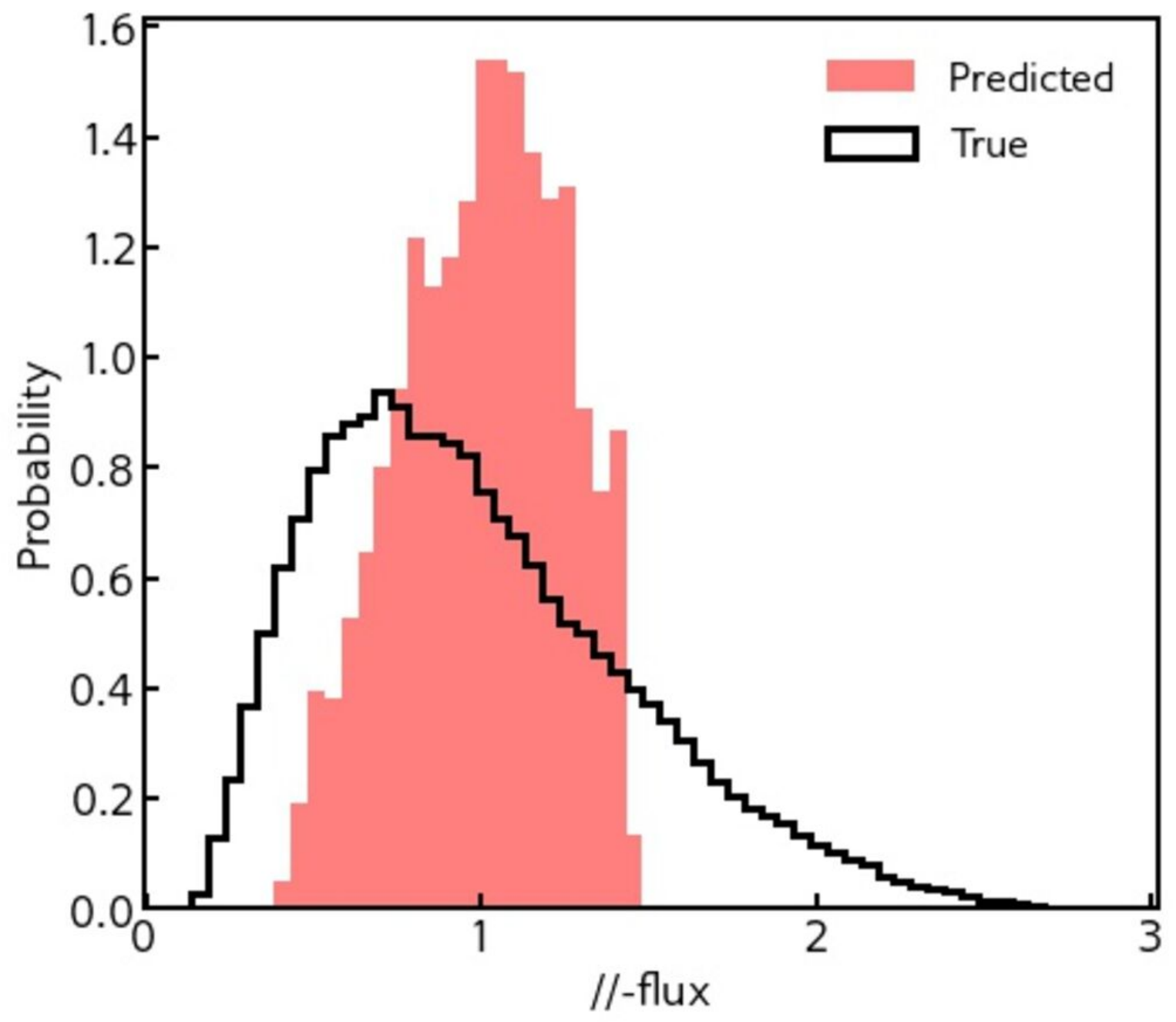

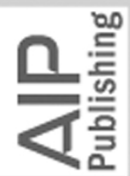




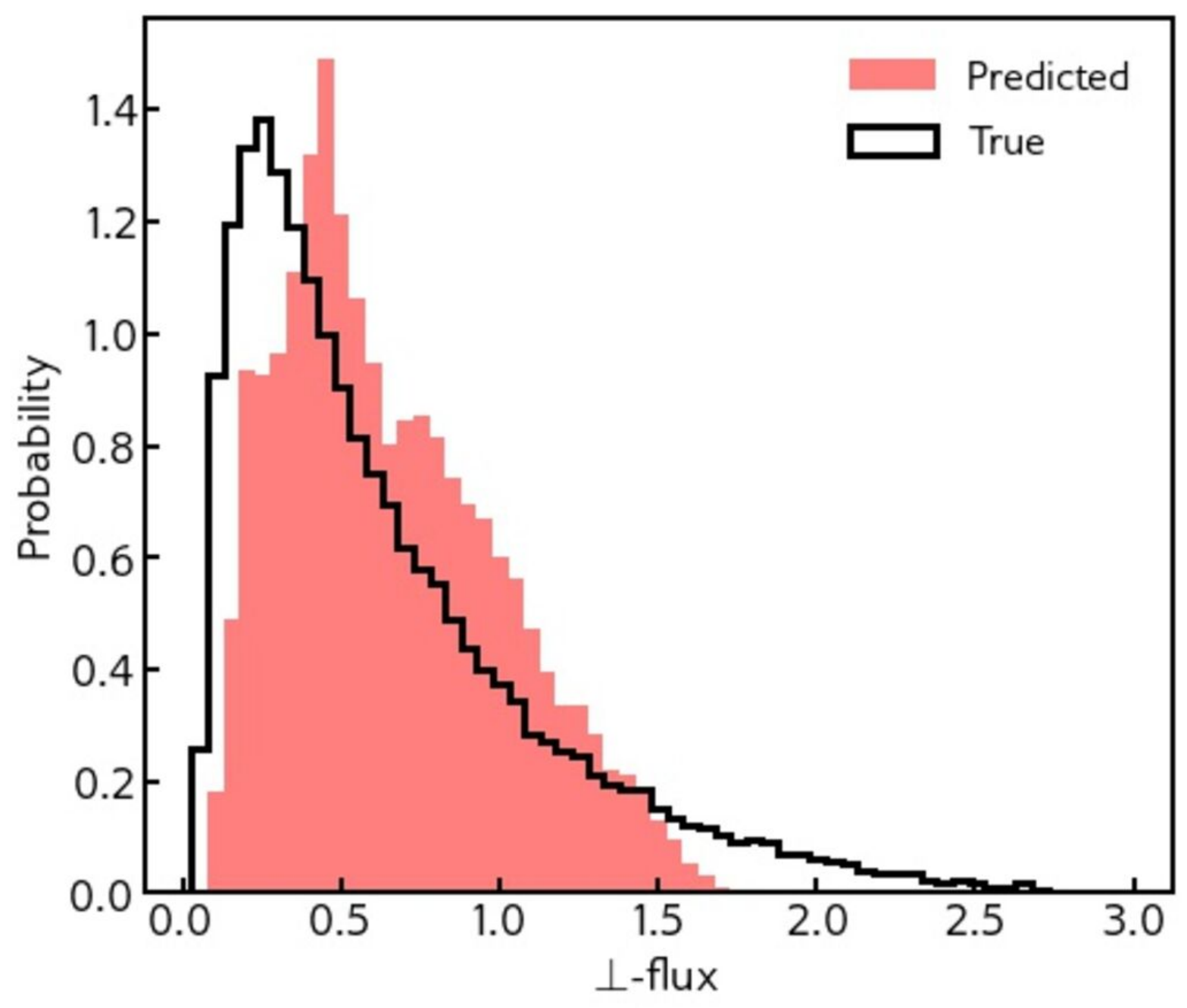

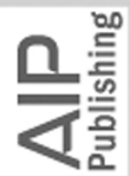




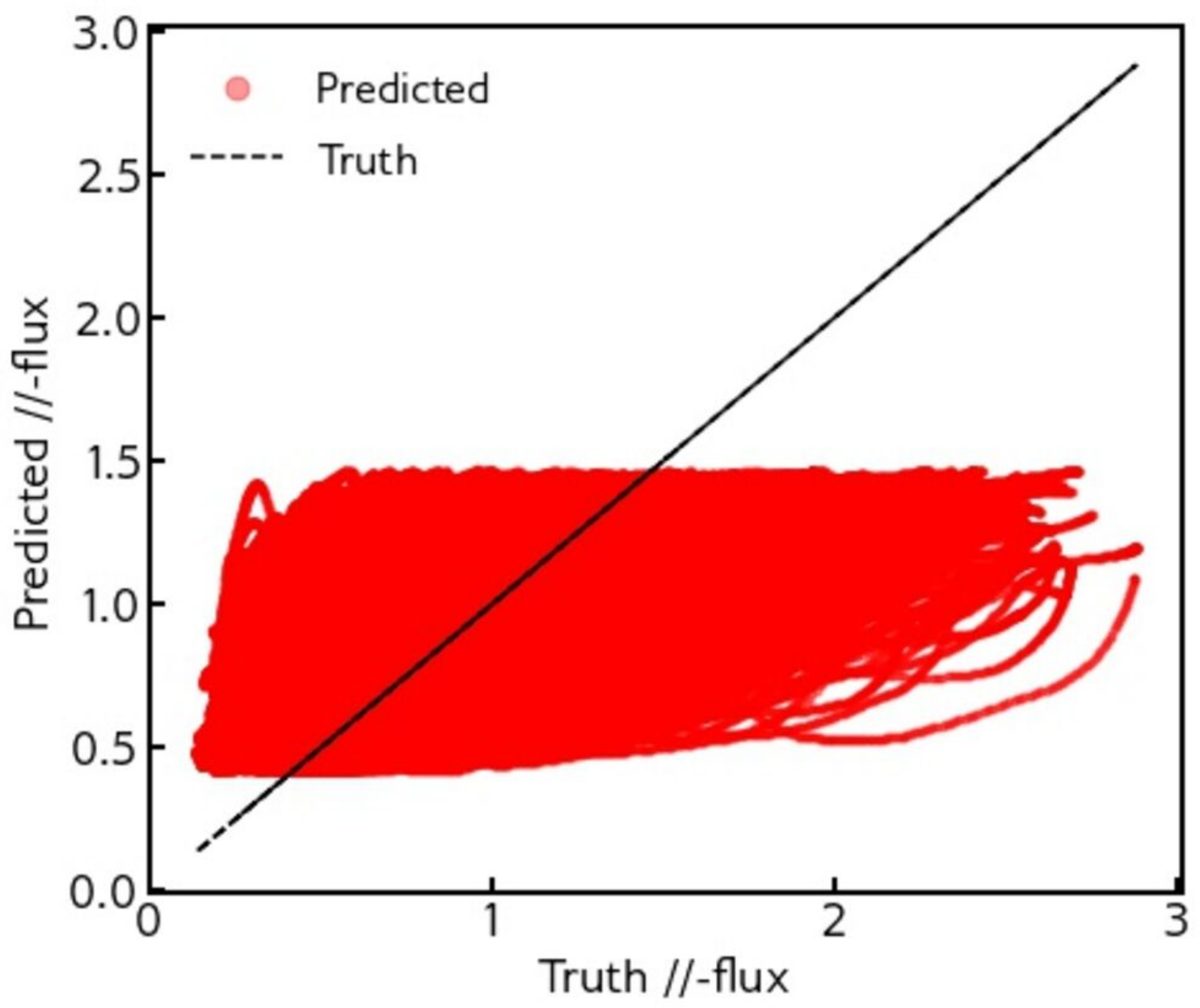

은 


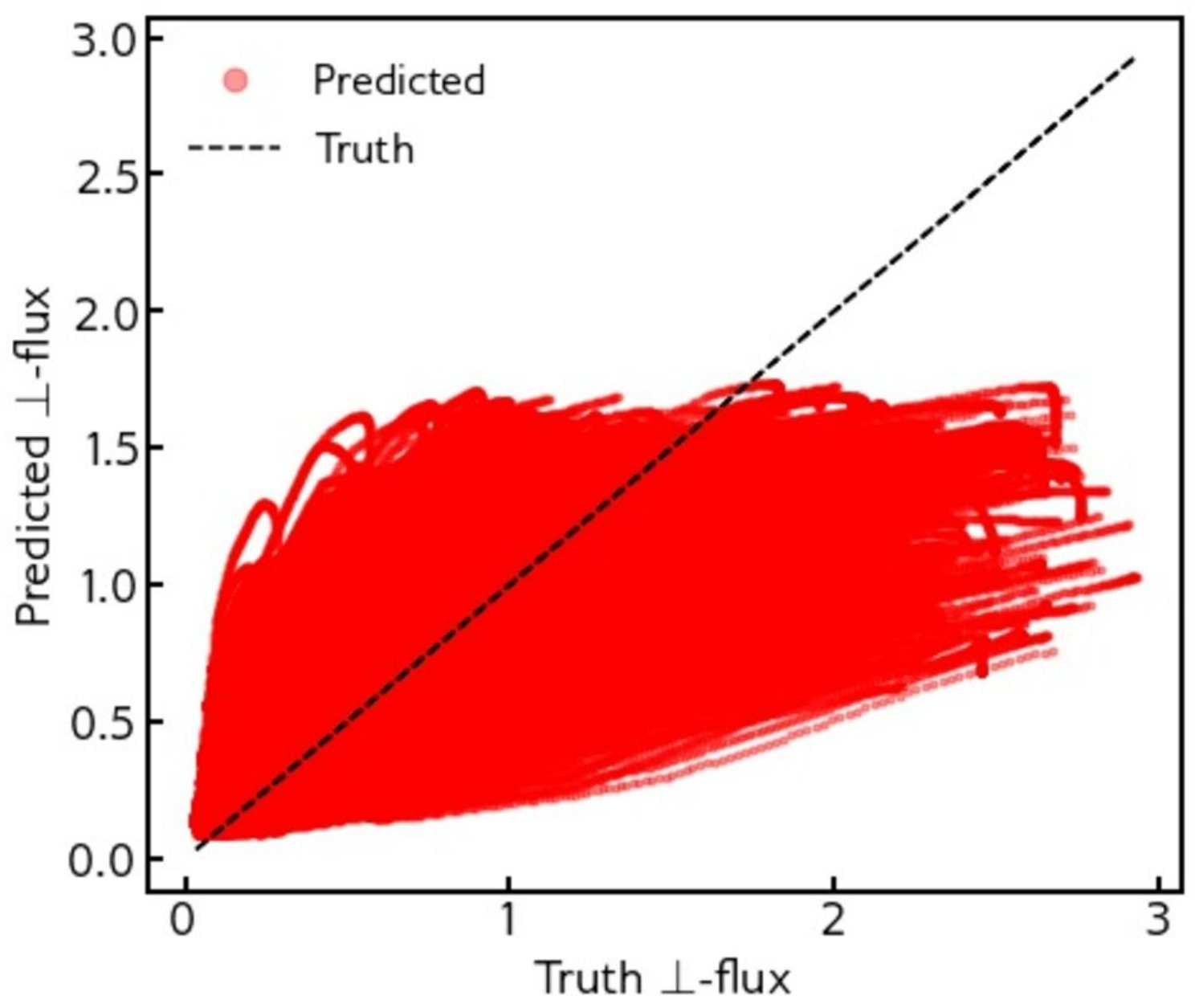

兄耪言 


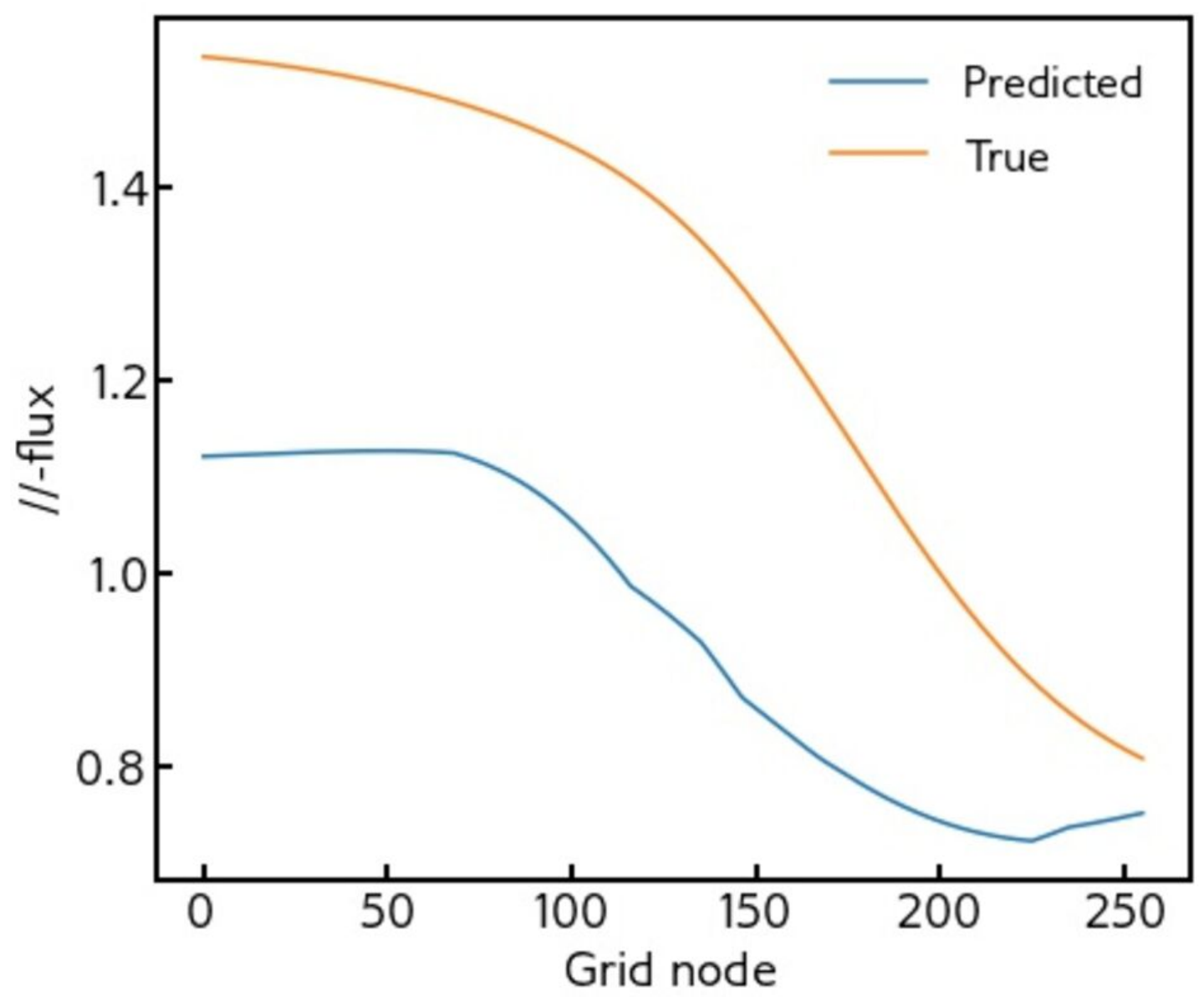

는 


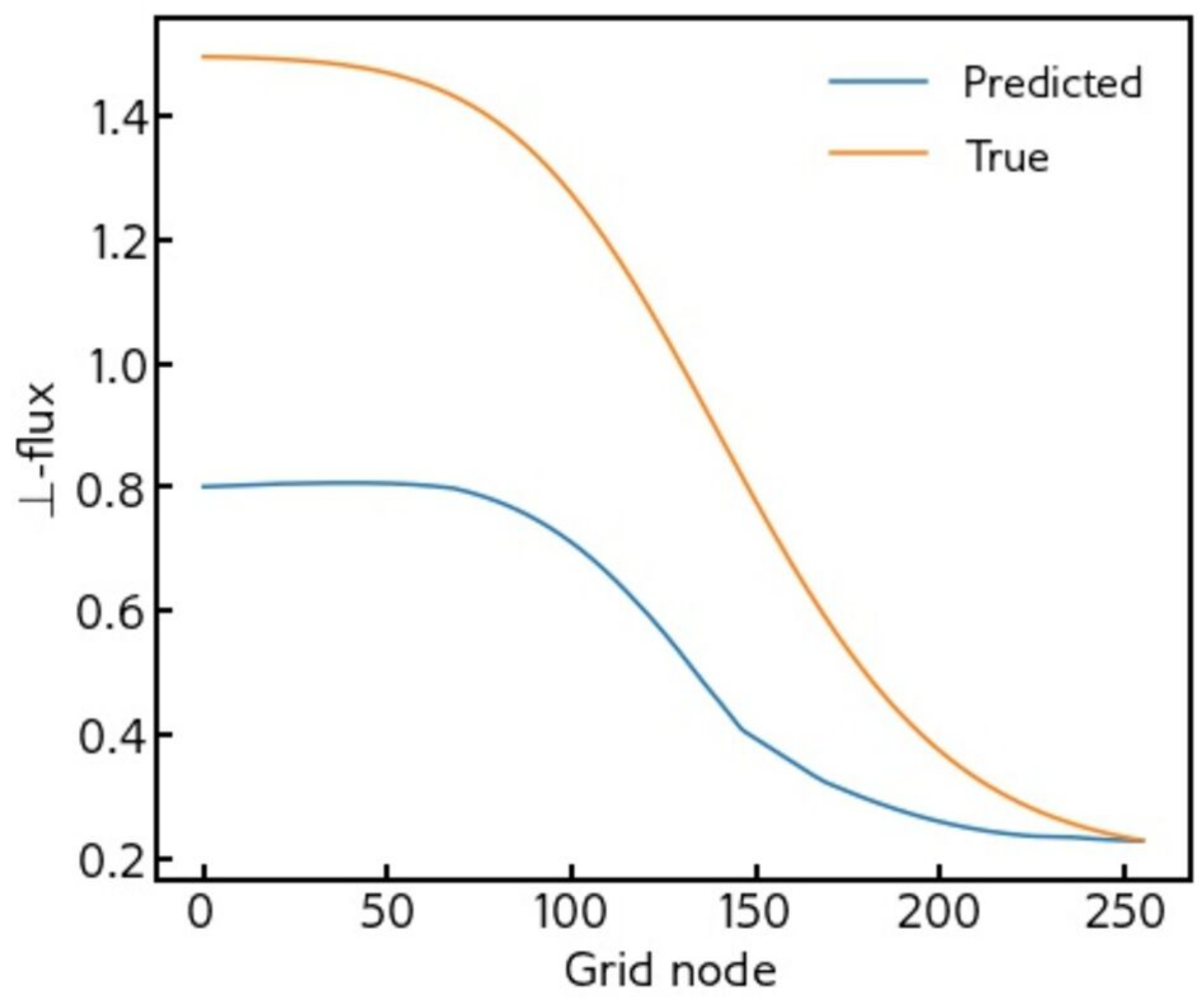

는 


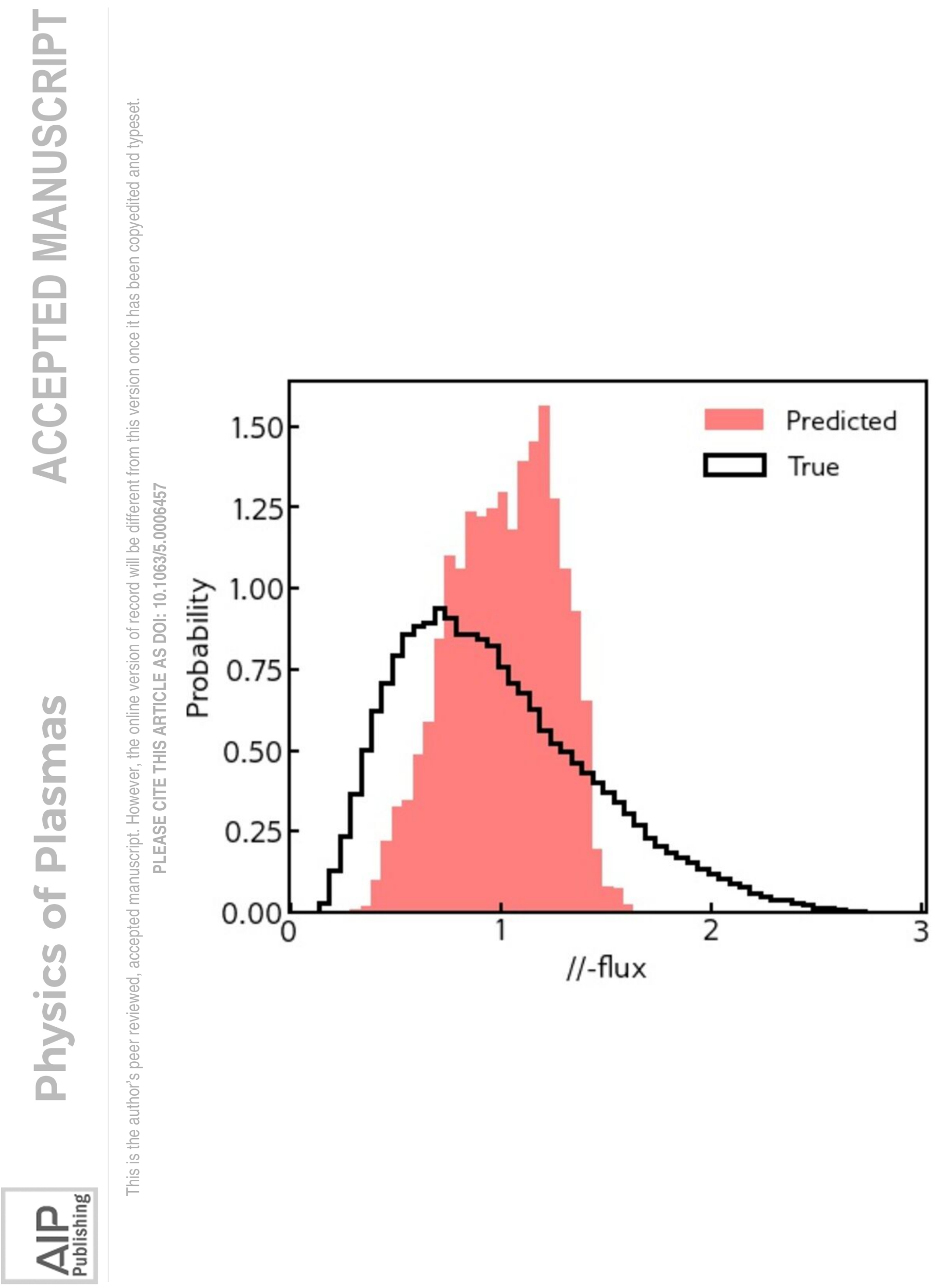




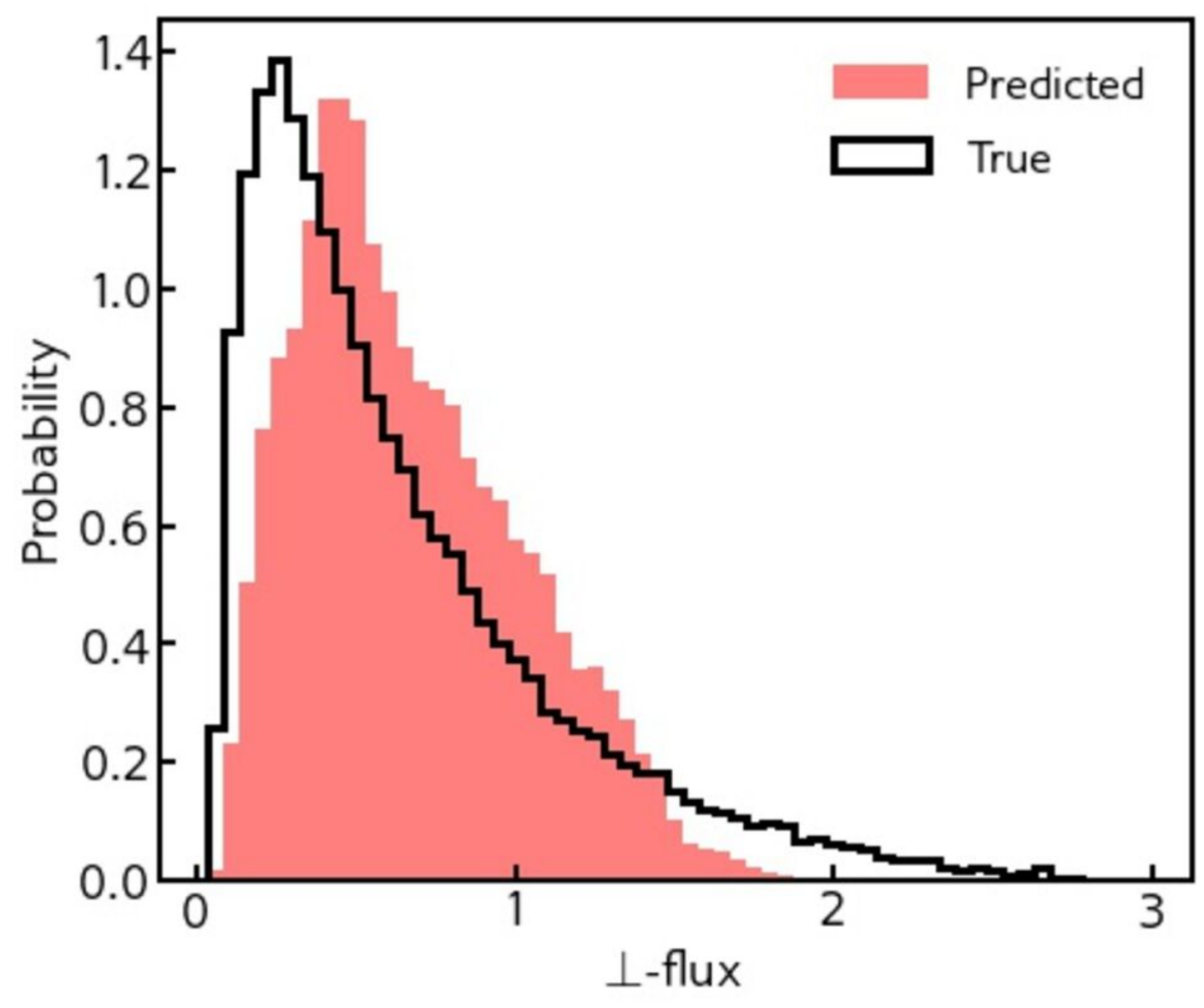

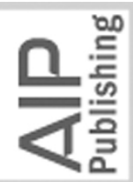




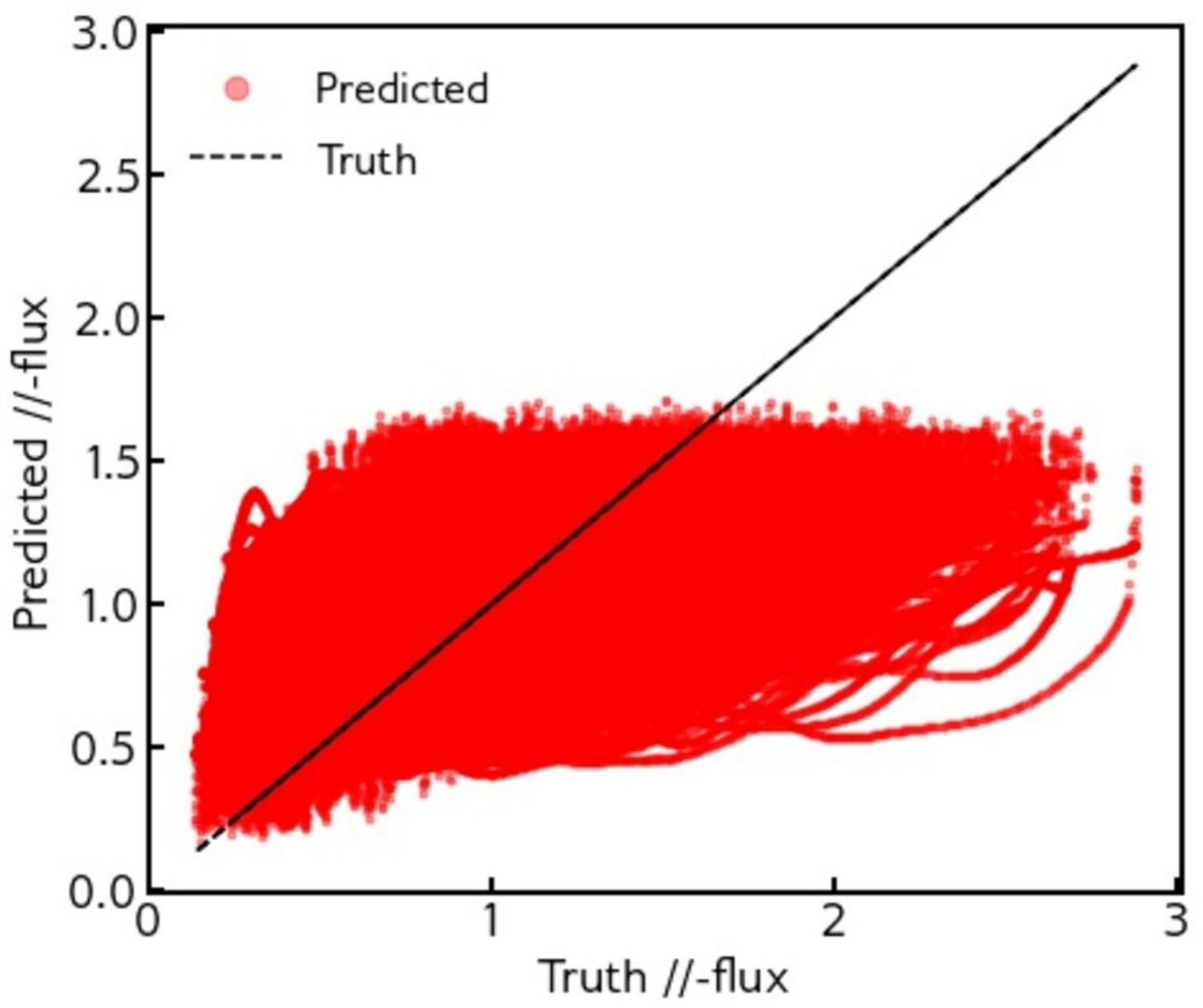

은 


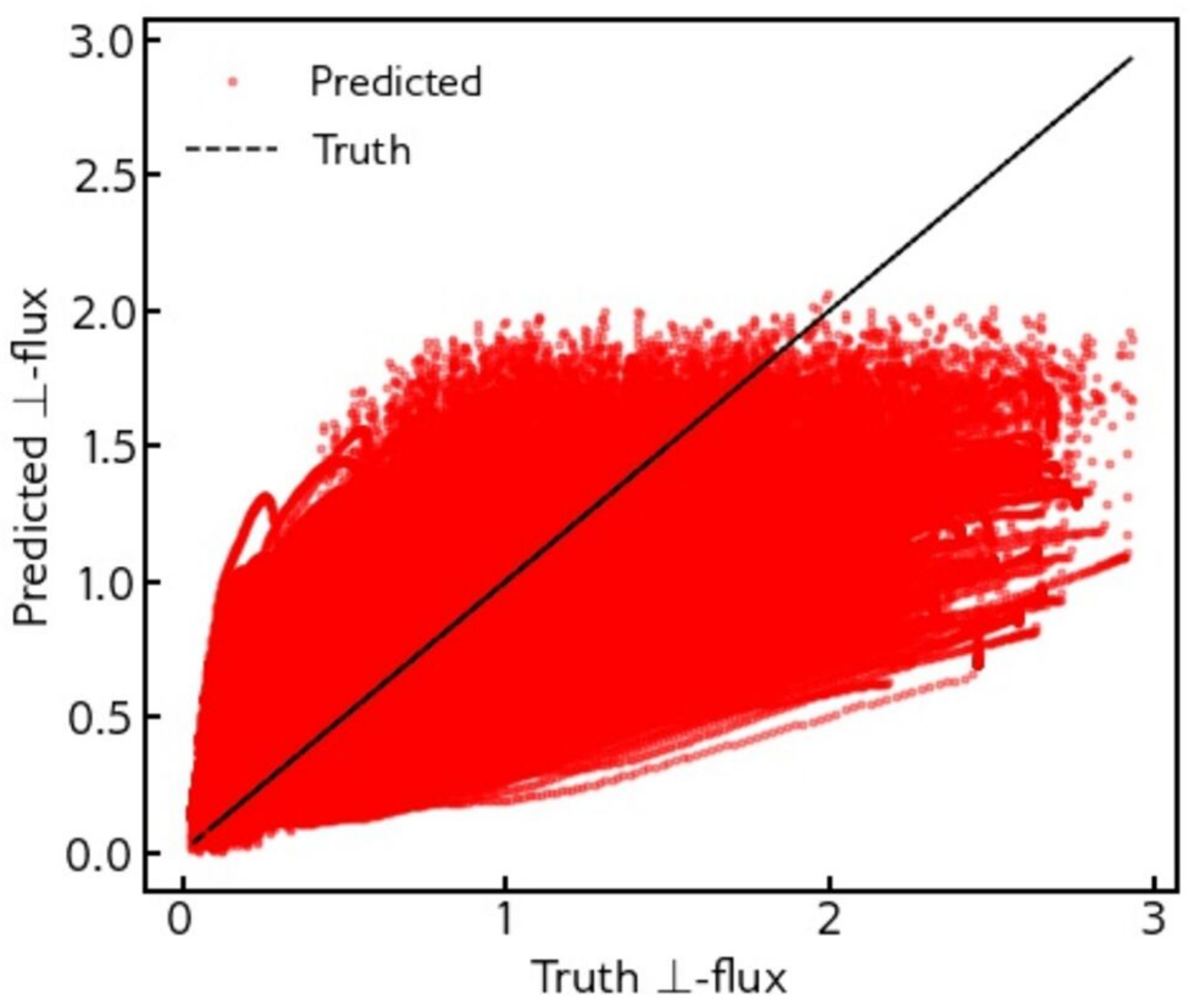

兄耪言 


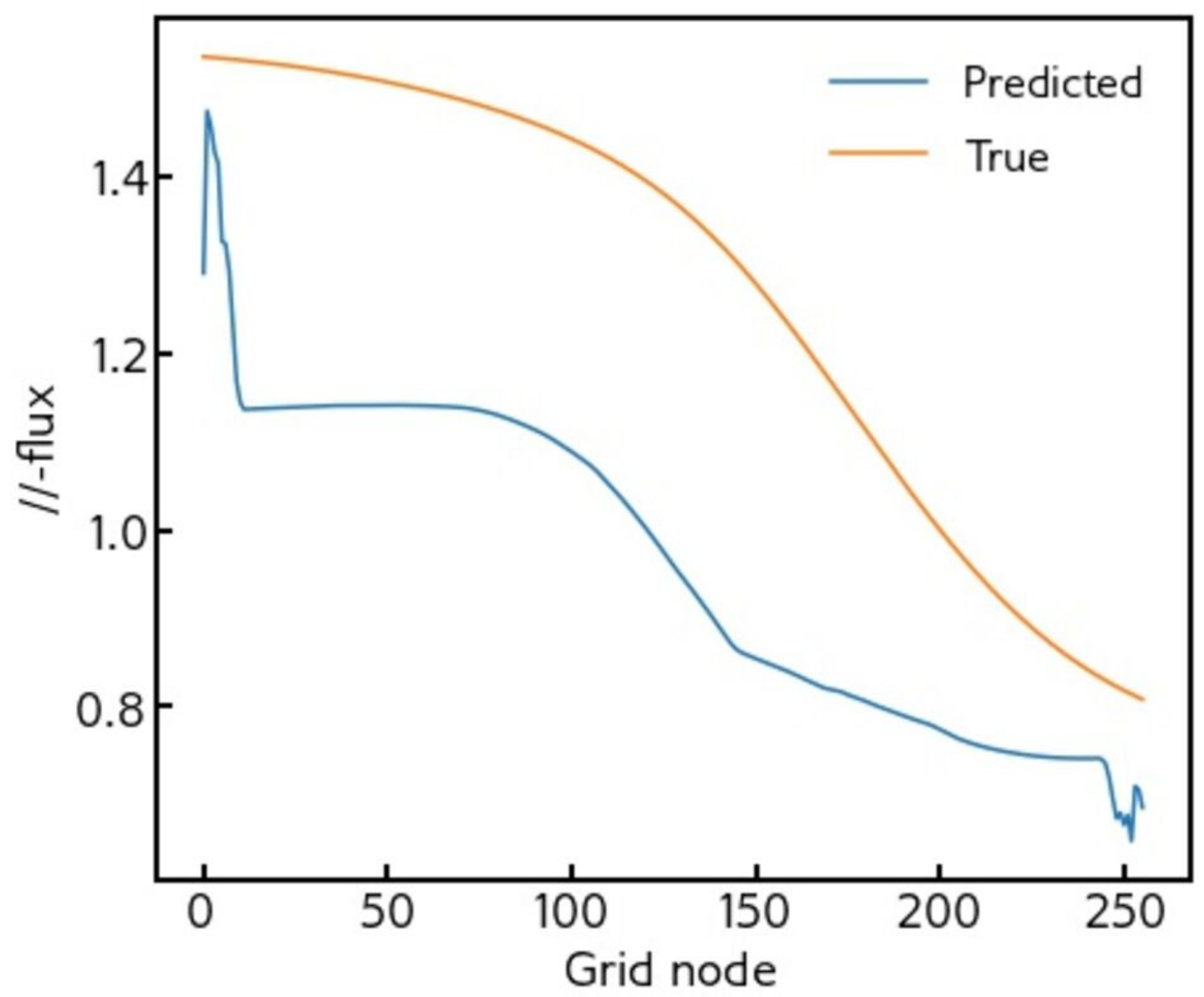

는 


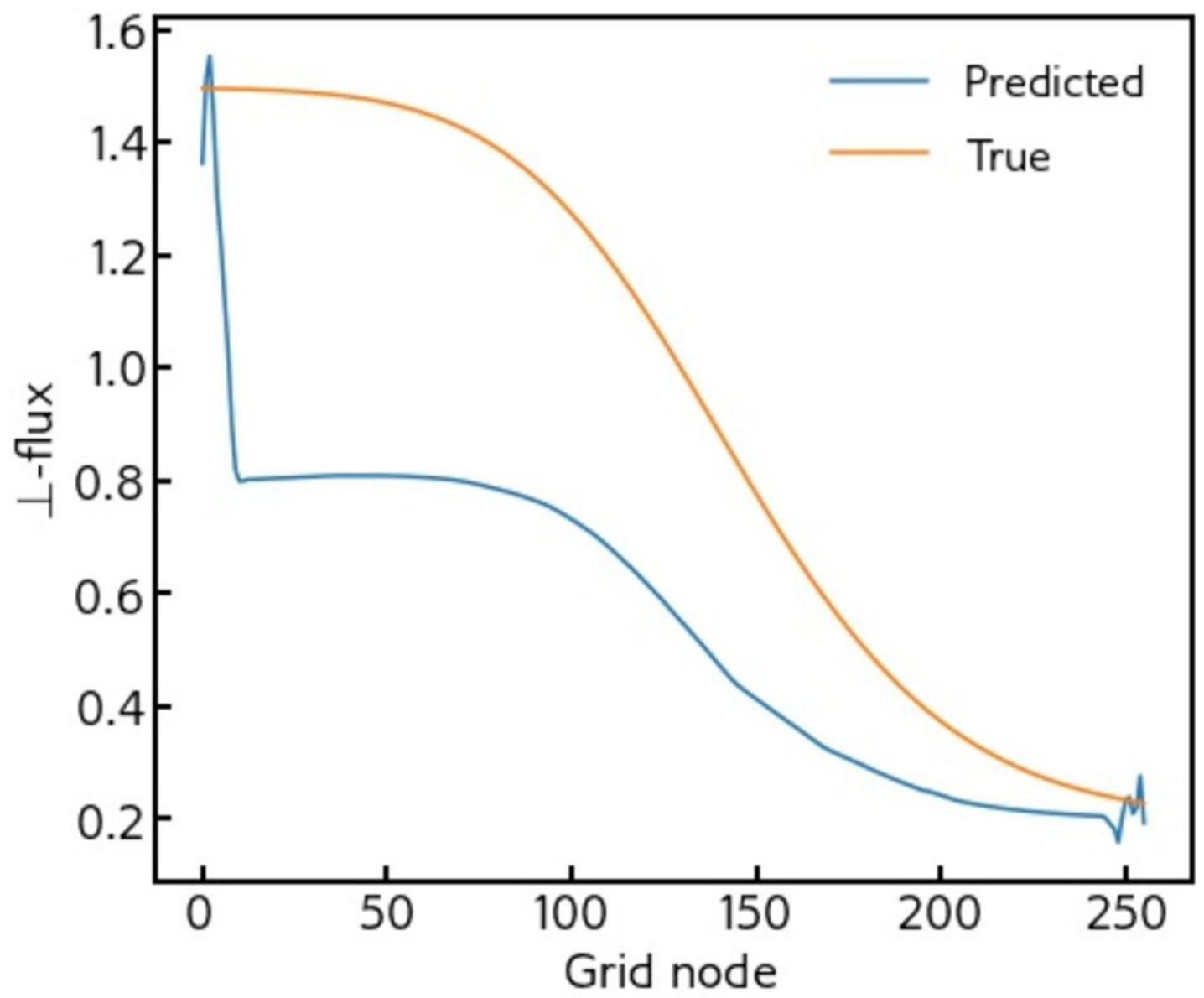

는 


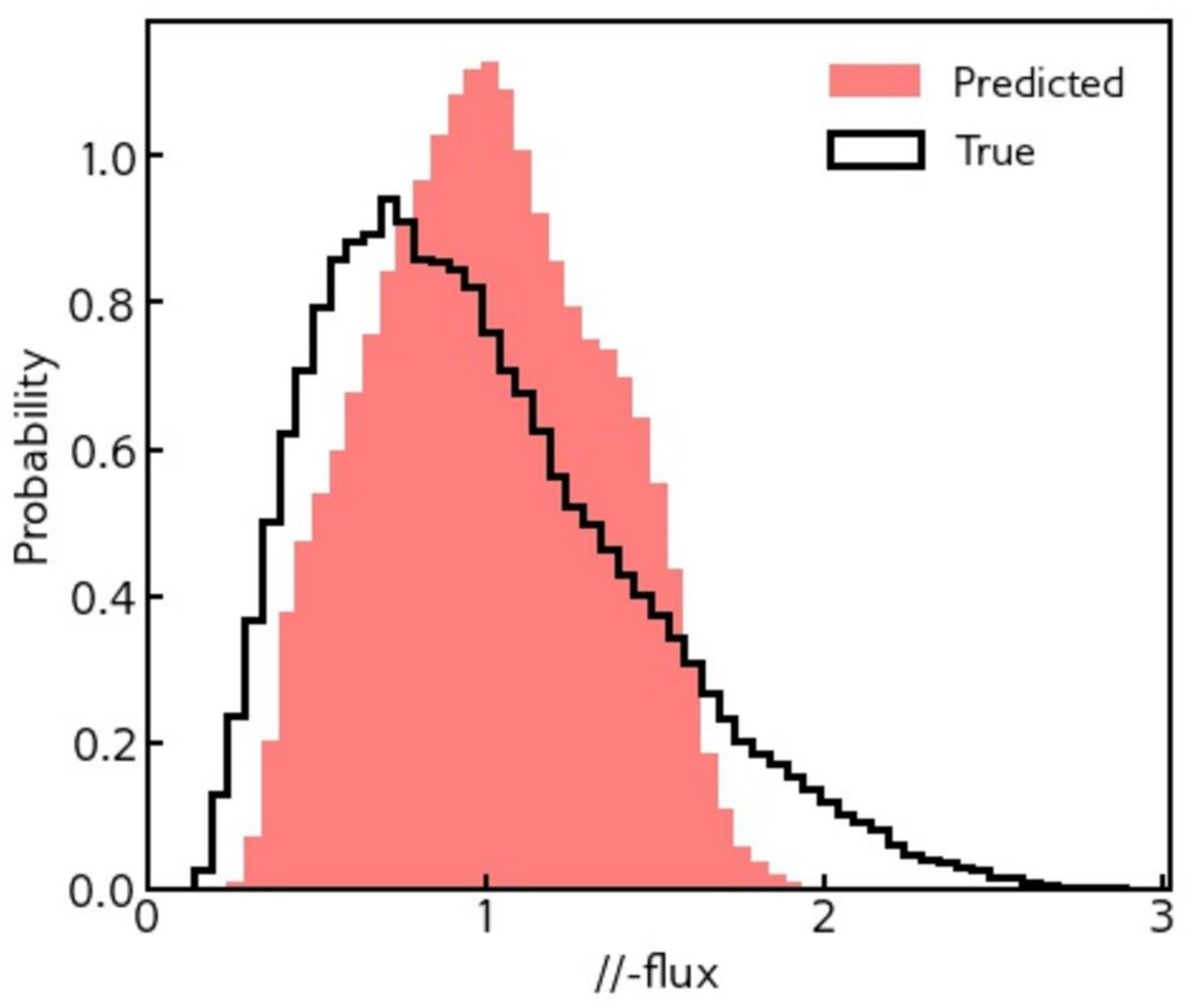

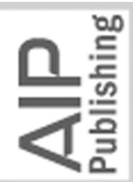




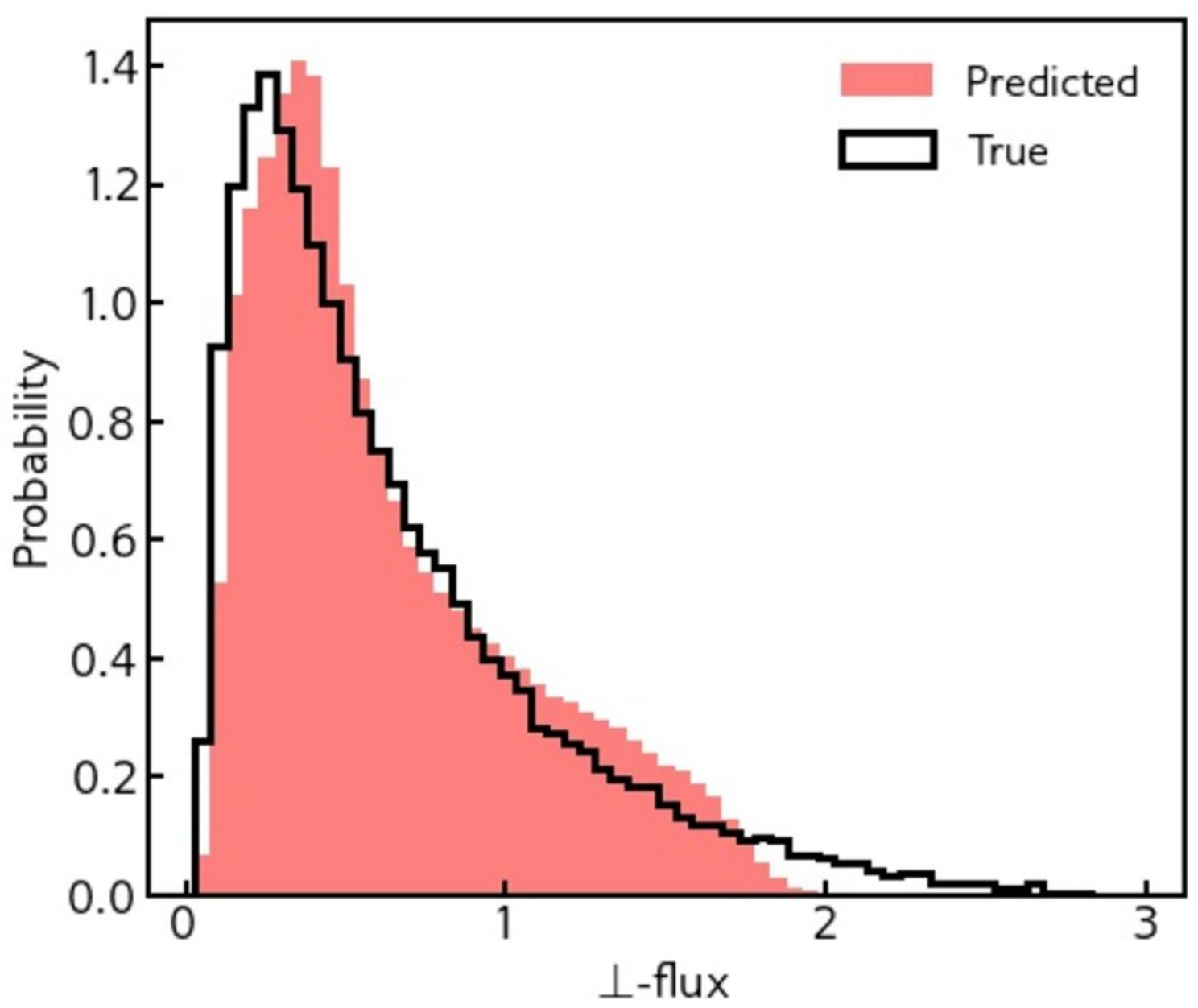

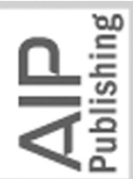




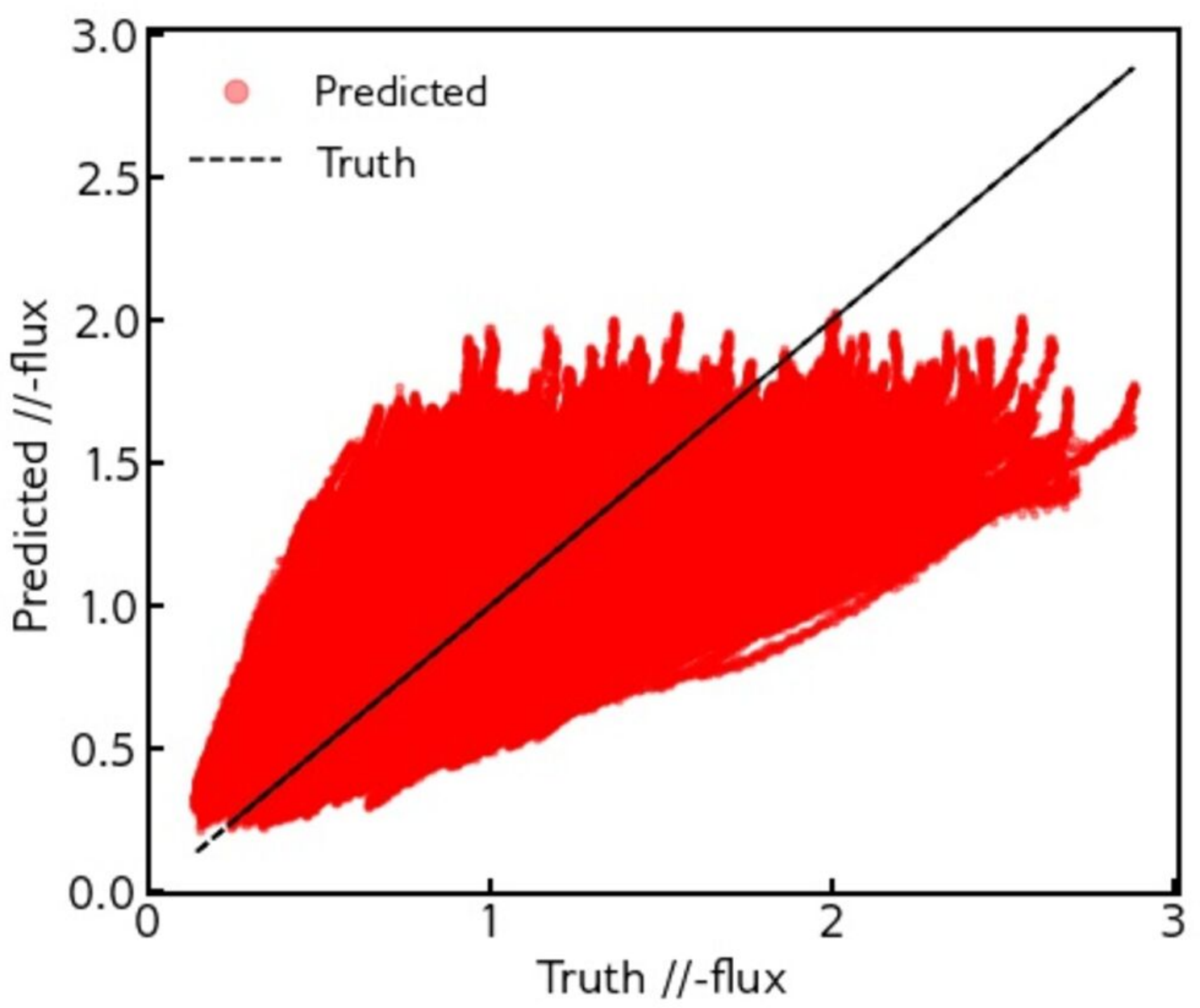

은 


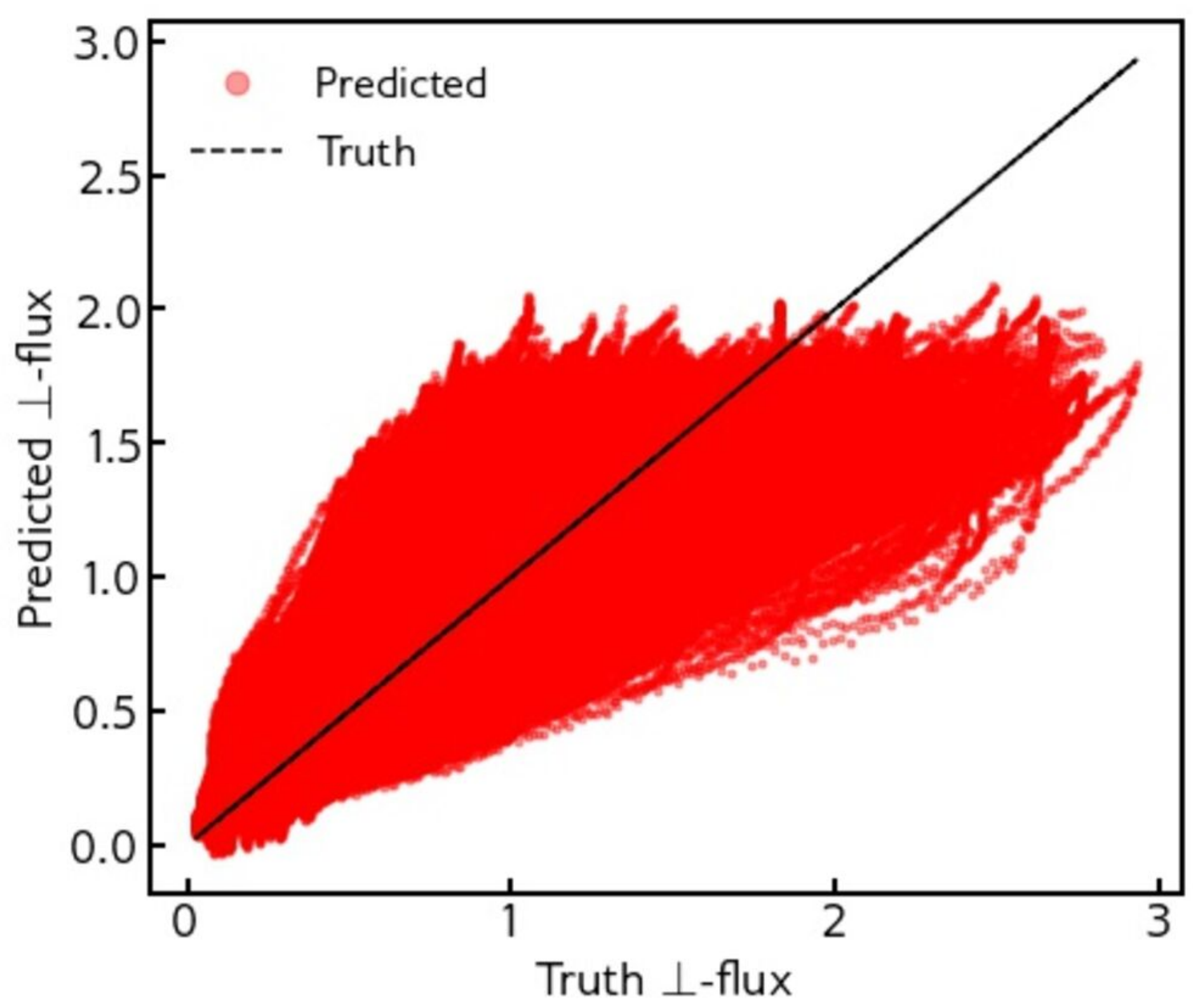

은 


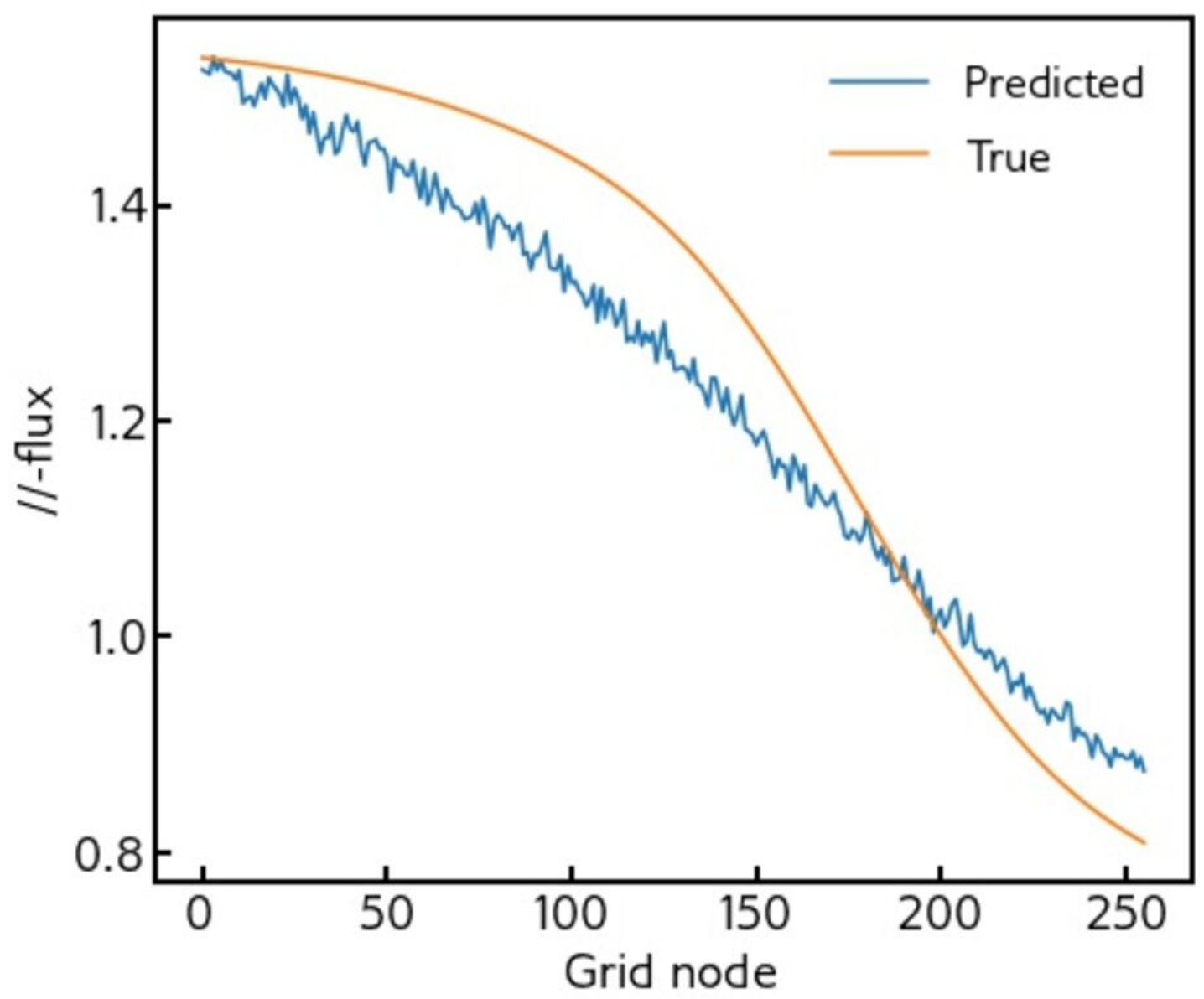

는 


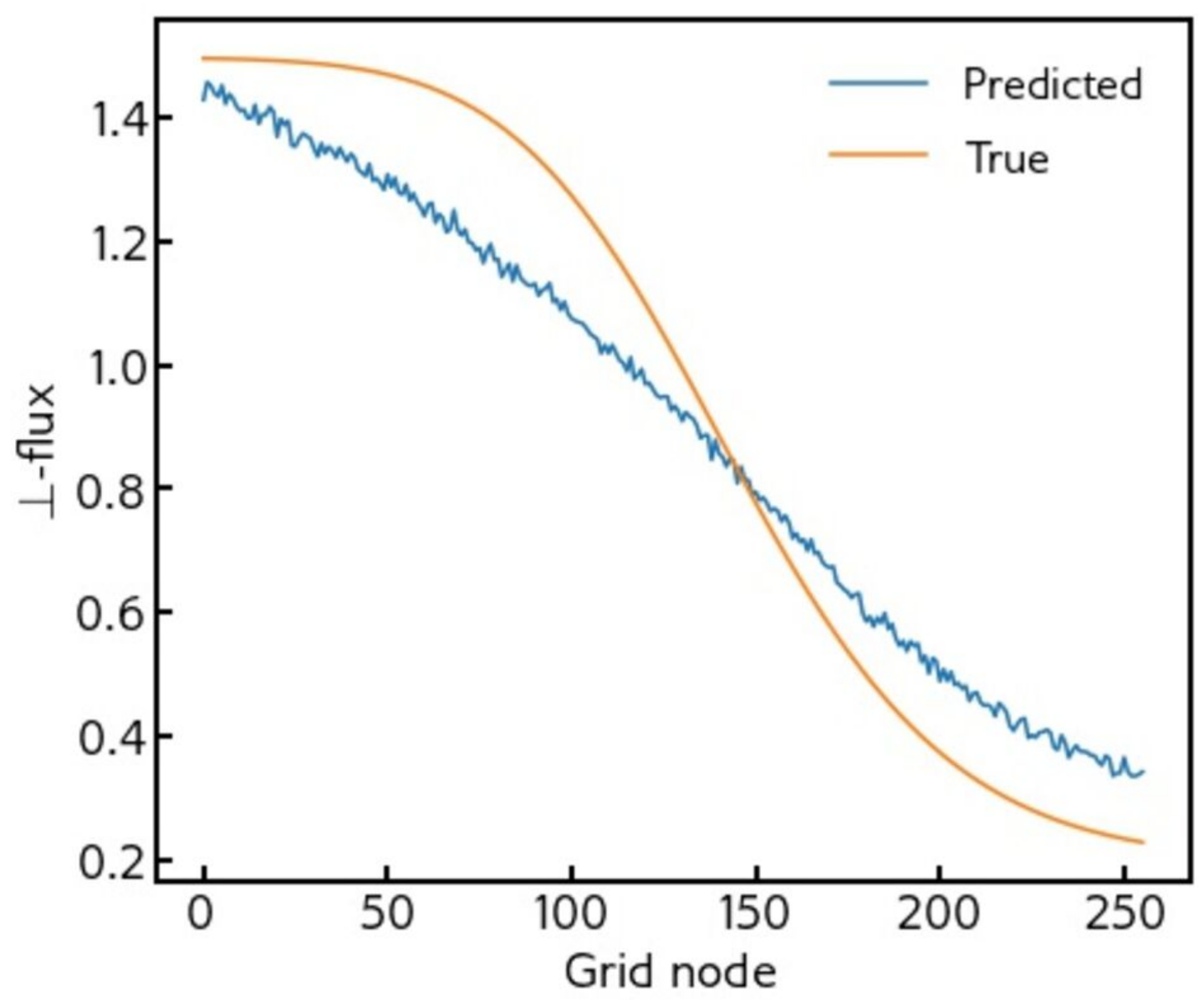

은 


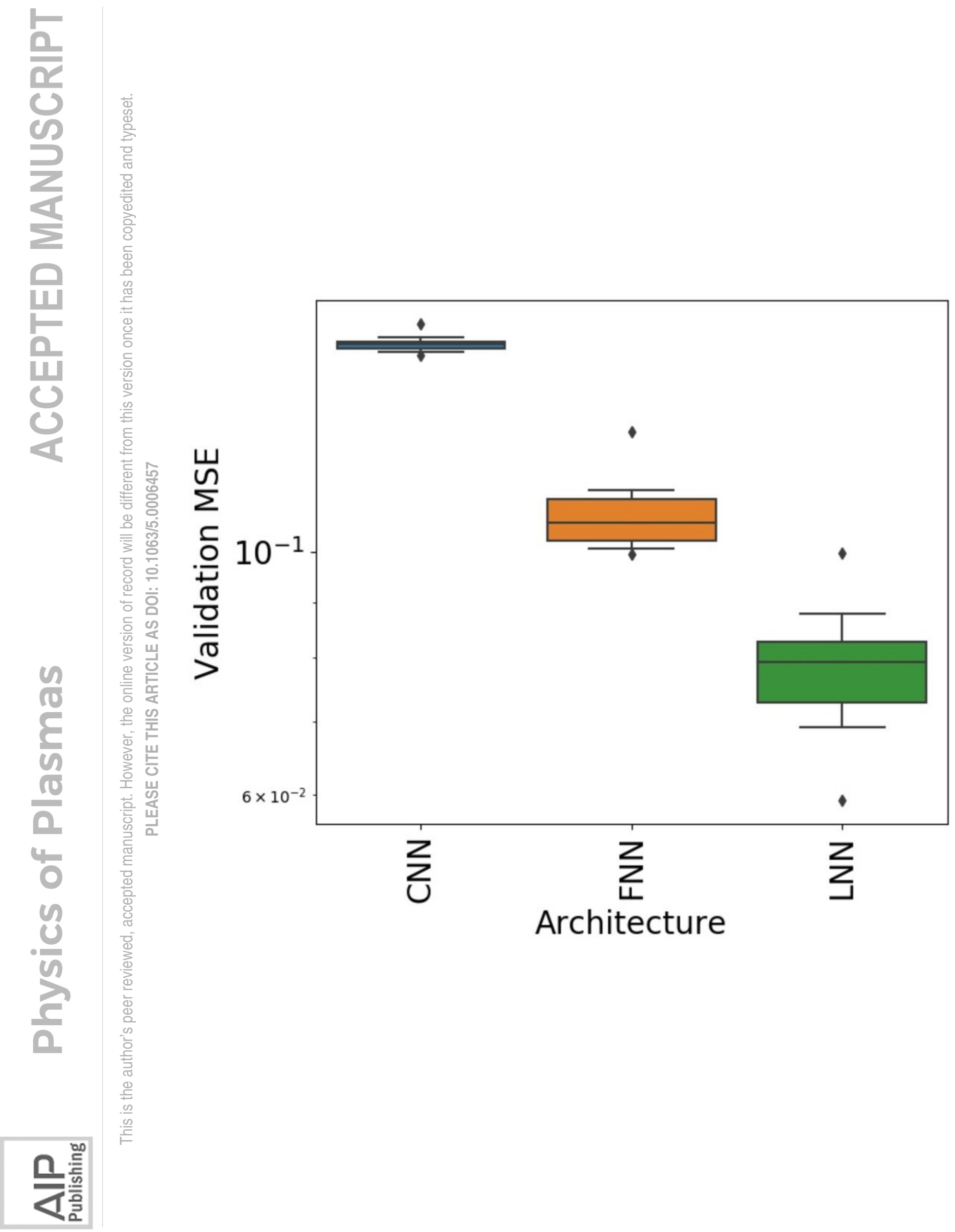




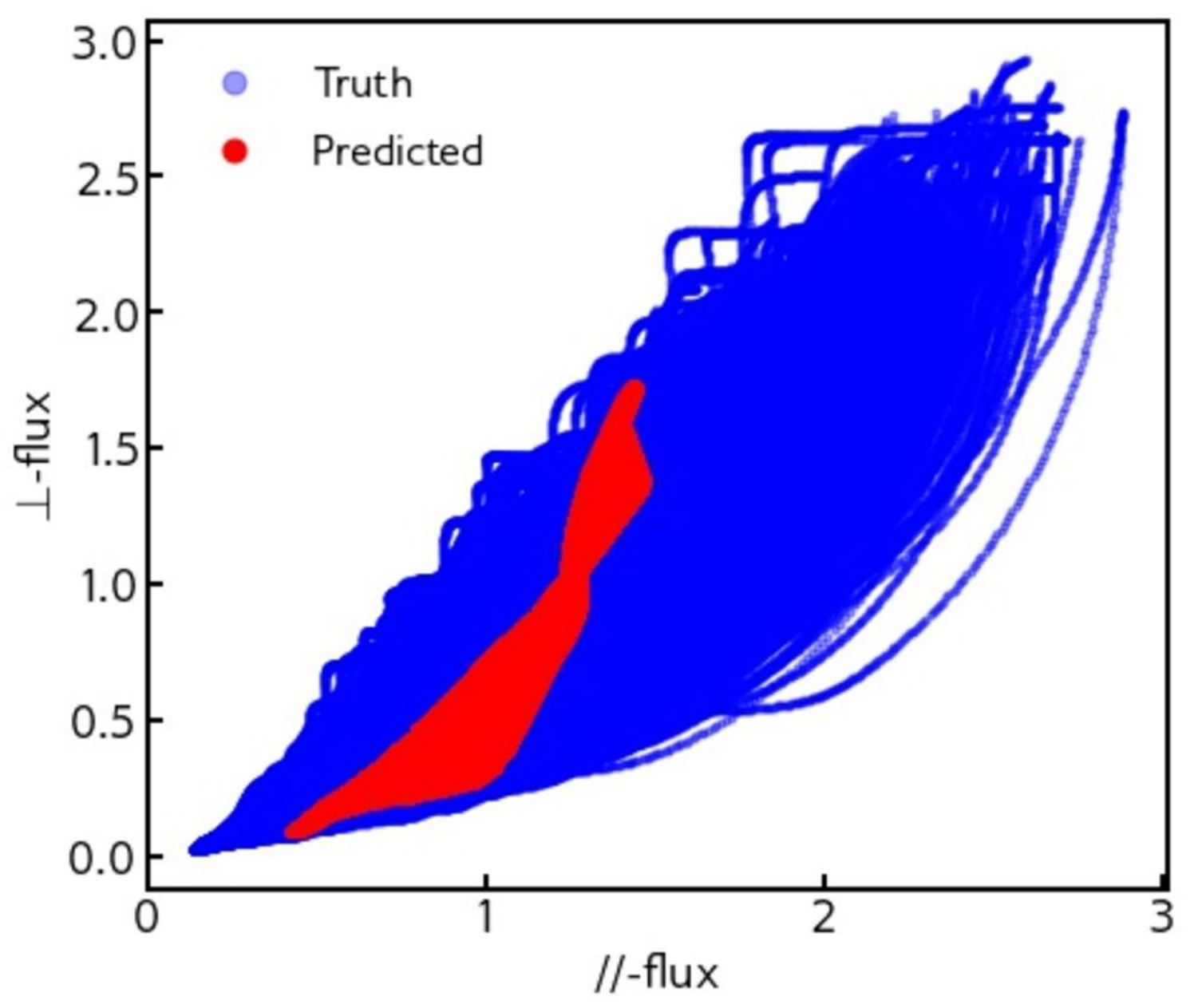

兄旁产 


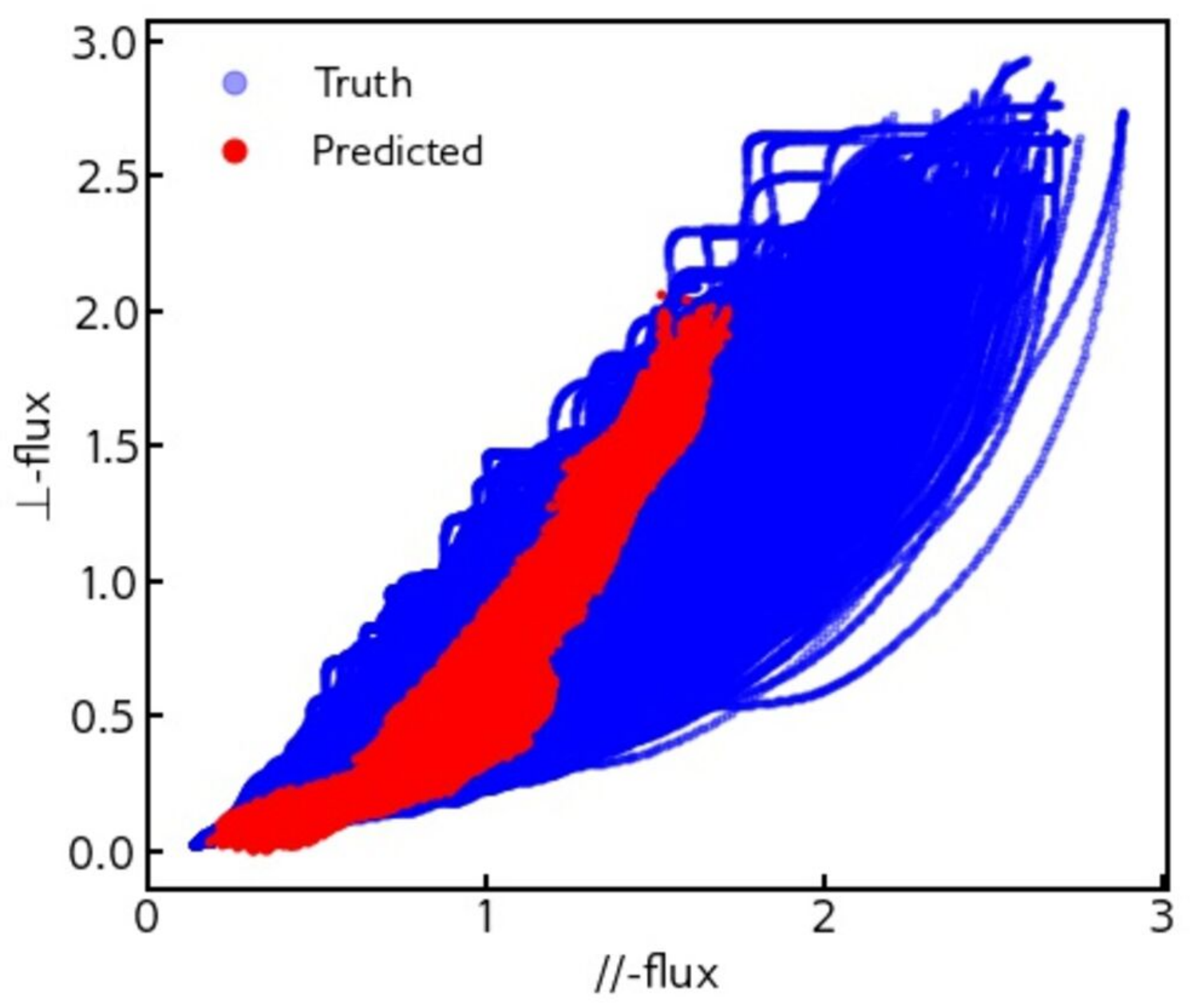

兄旁产 


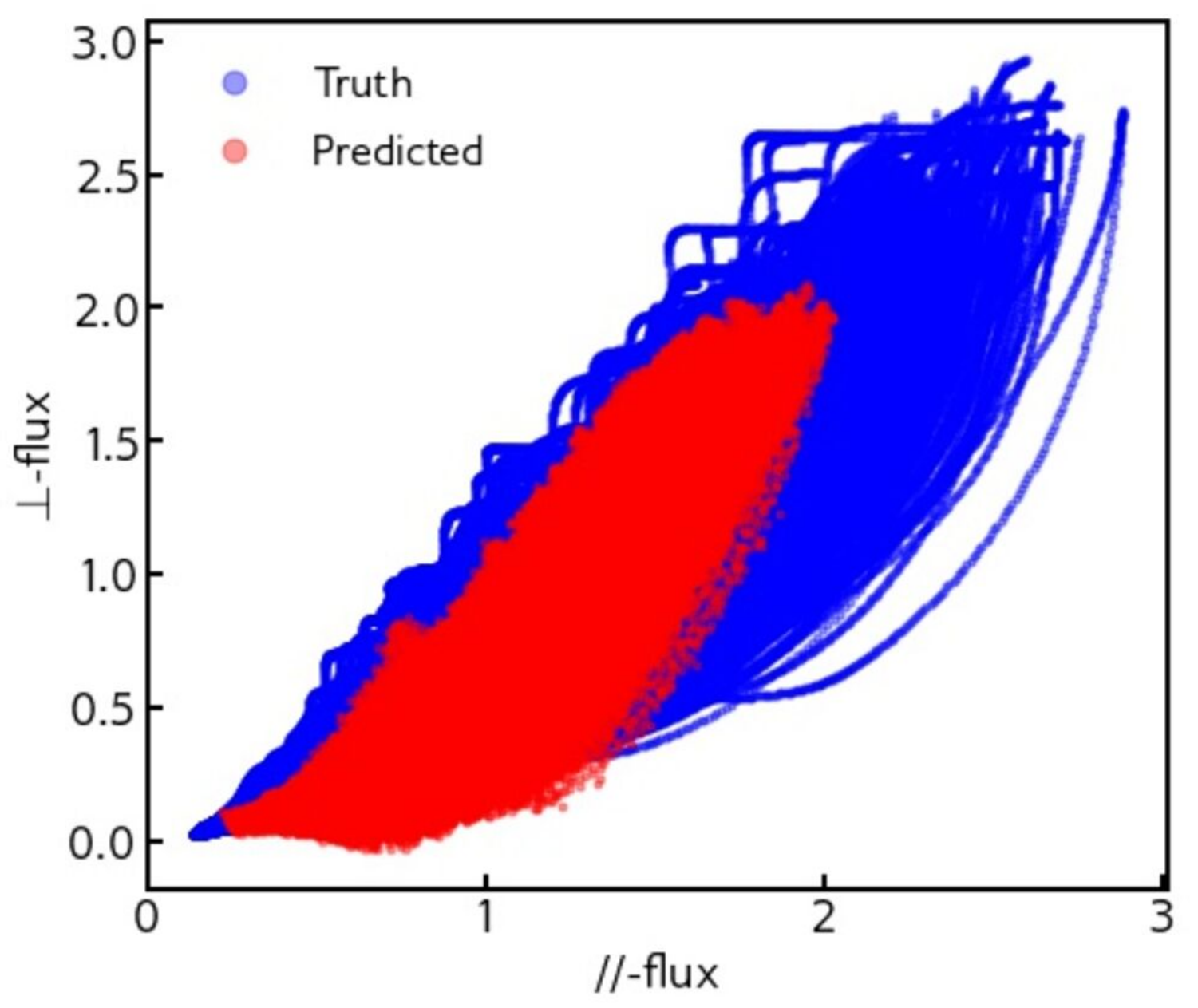

兄旁产 


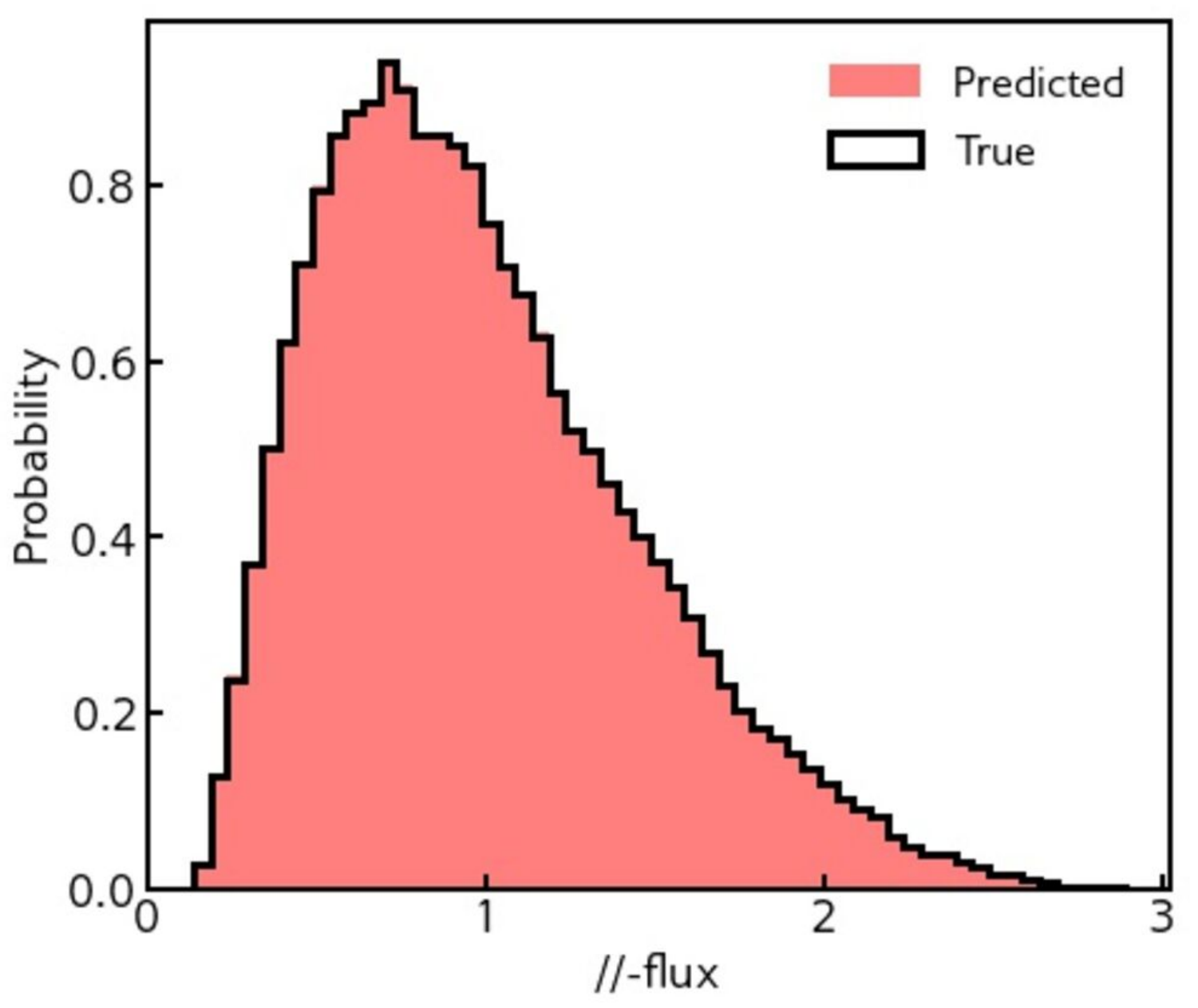

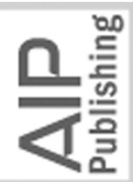




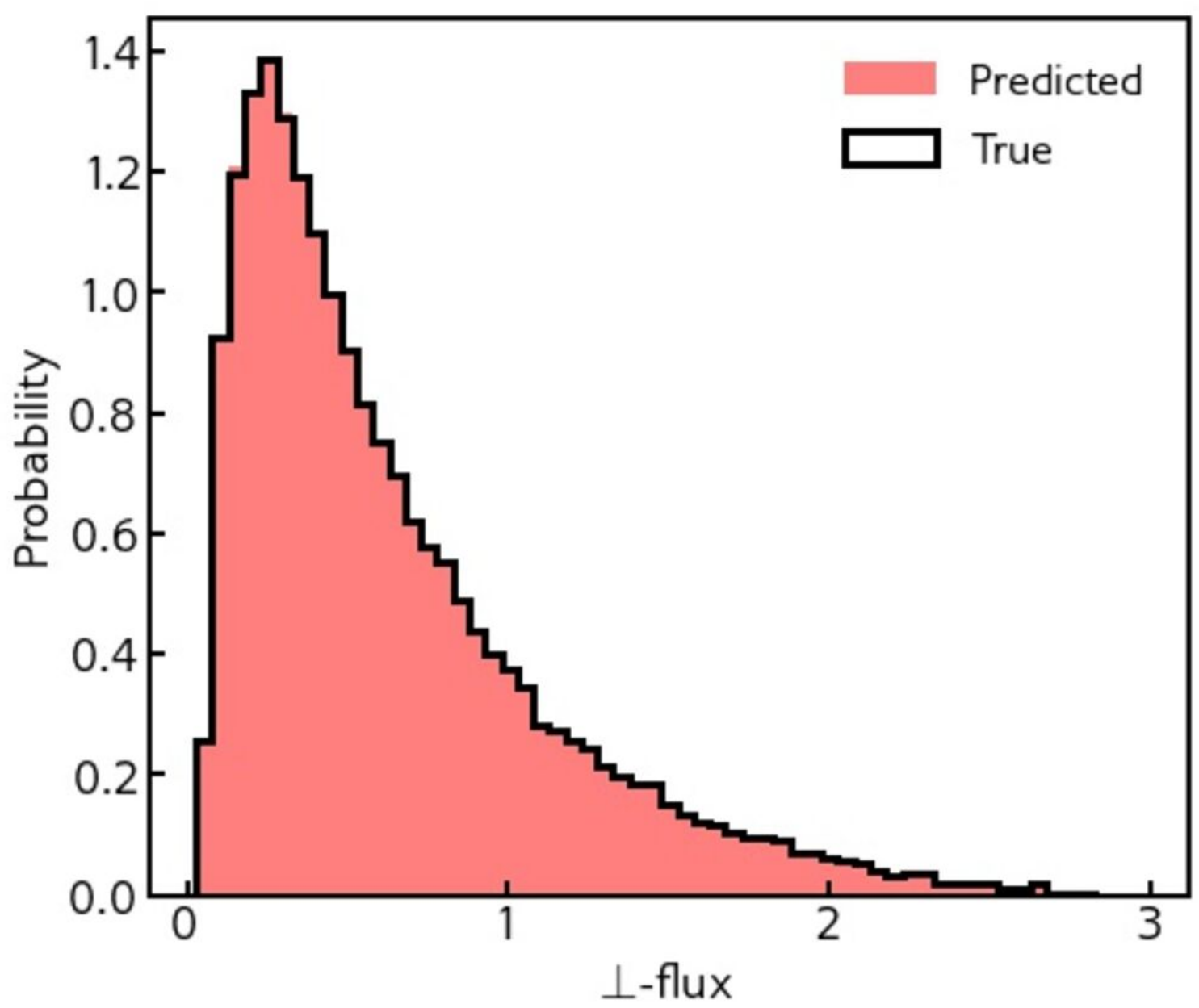

은 


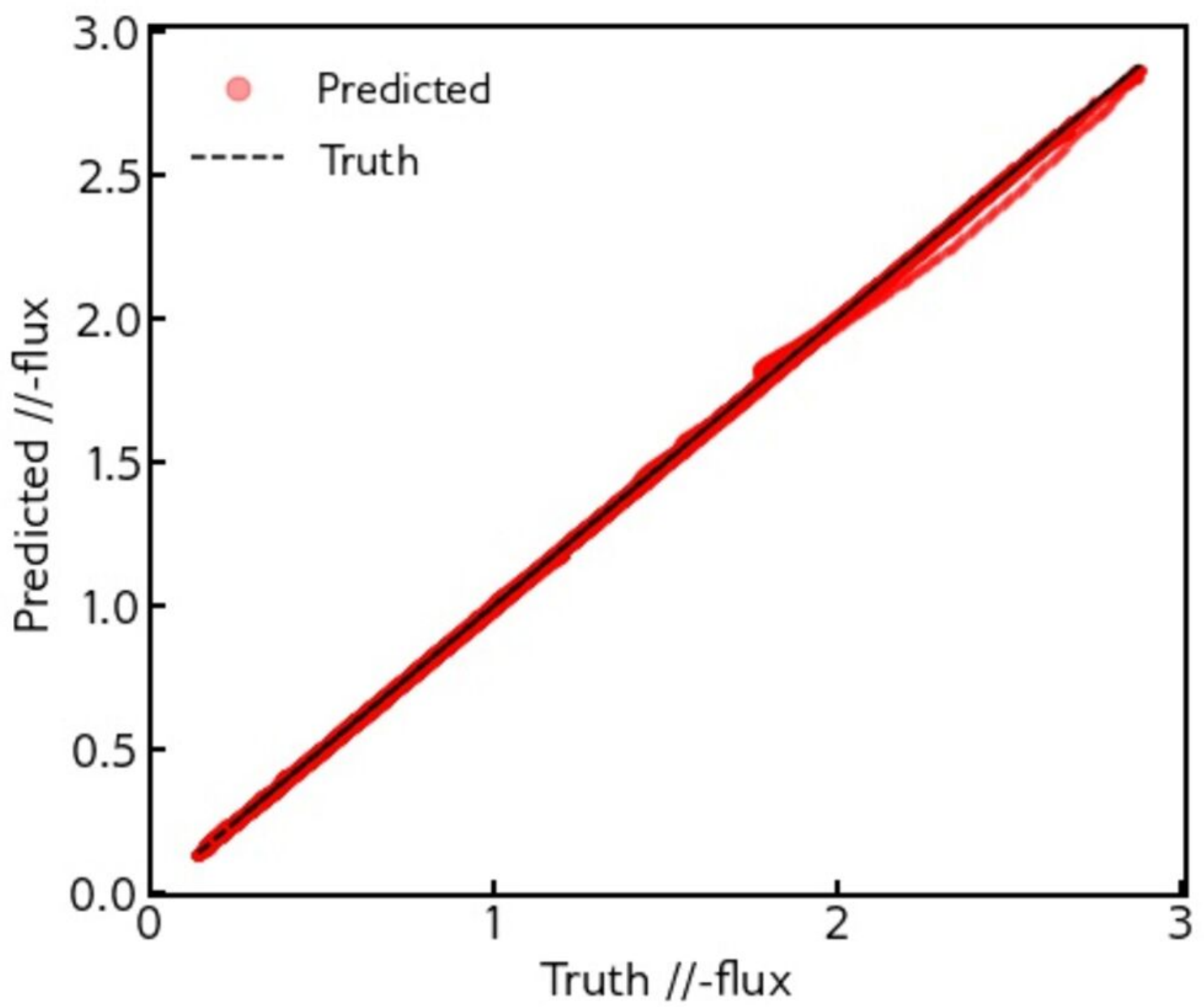

吅耪高 


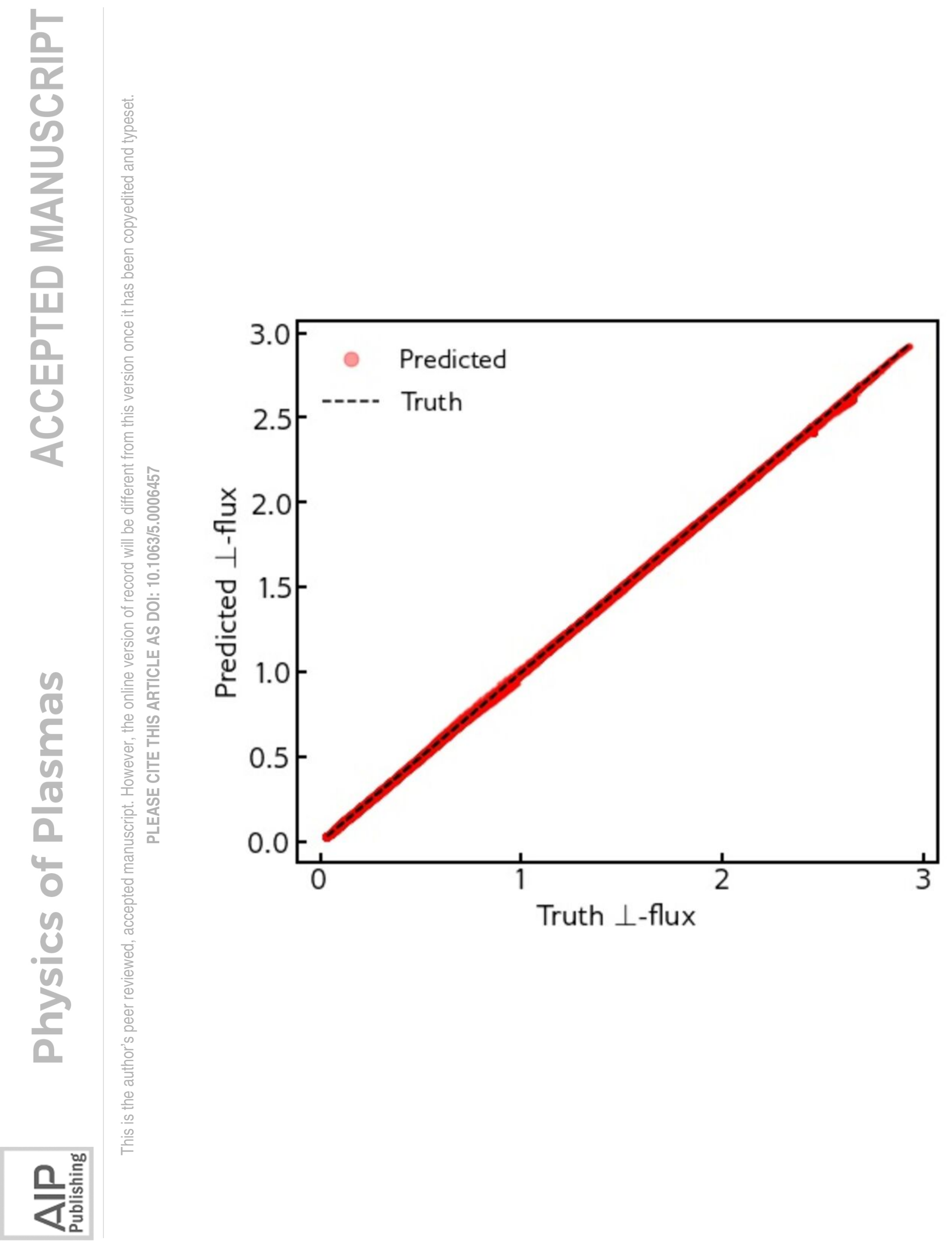




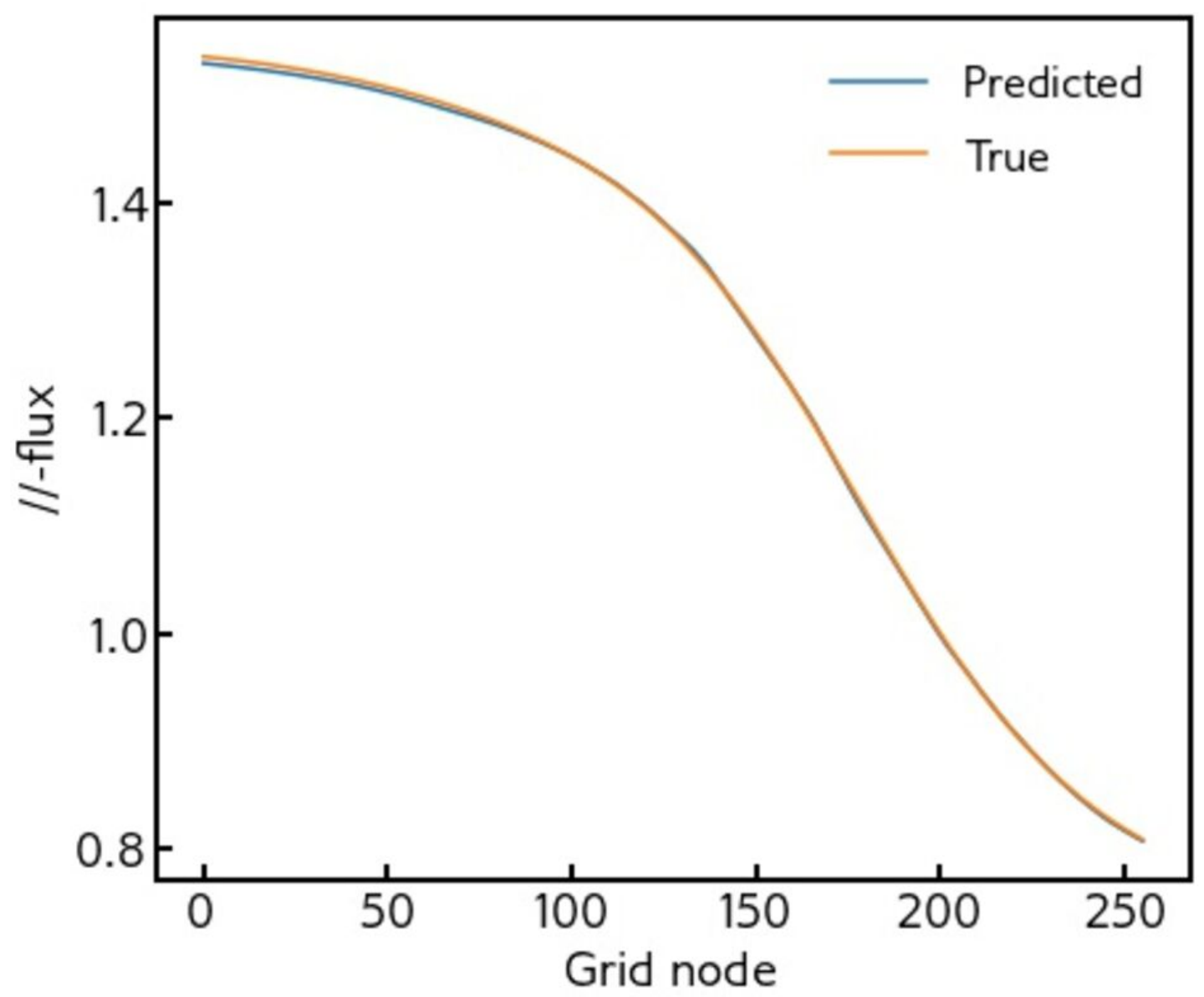

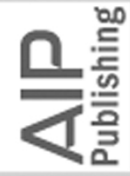




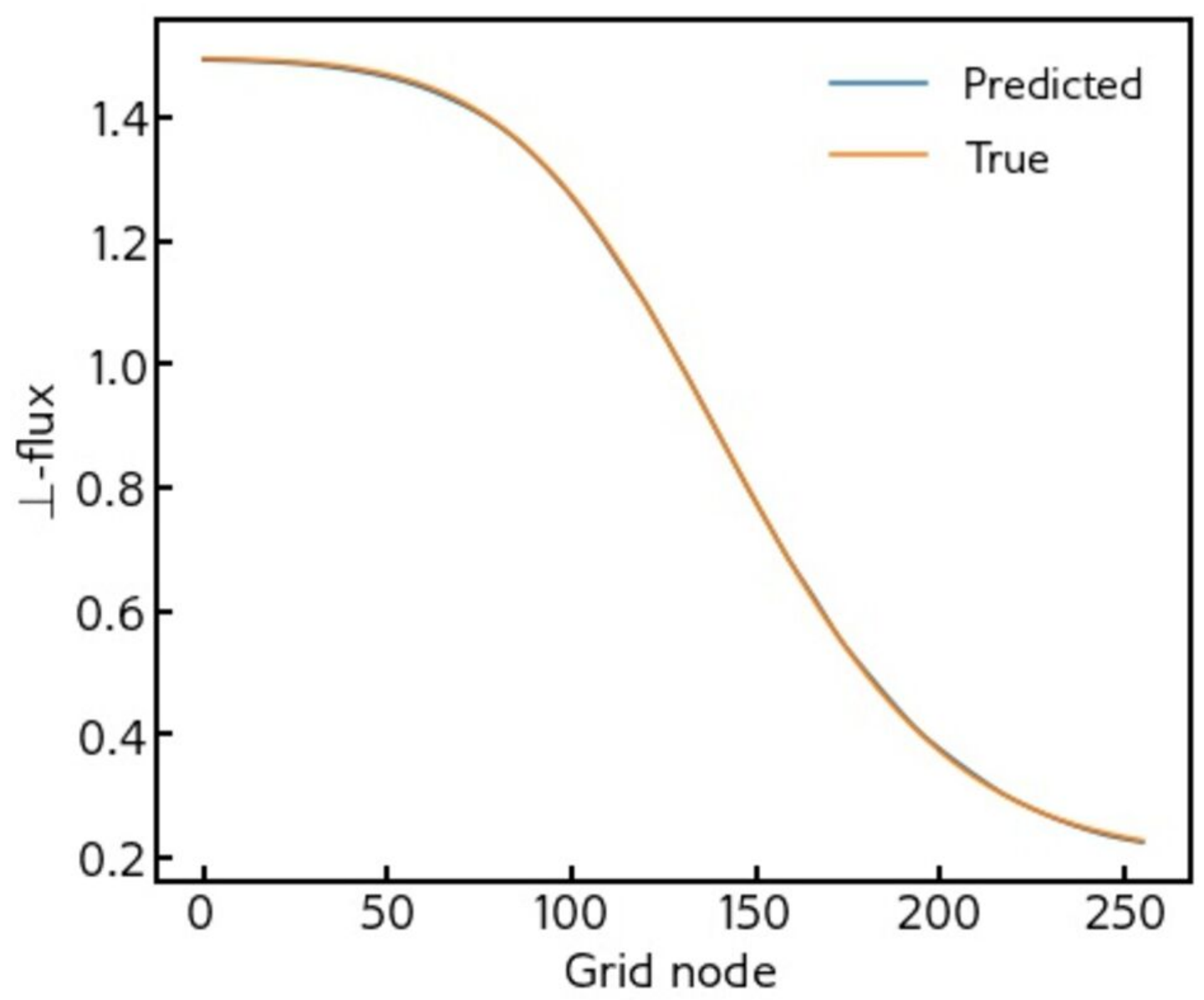

은 


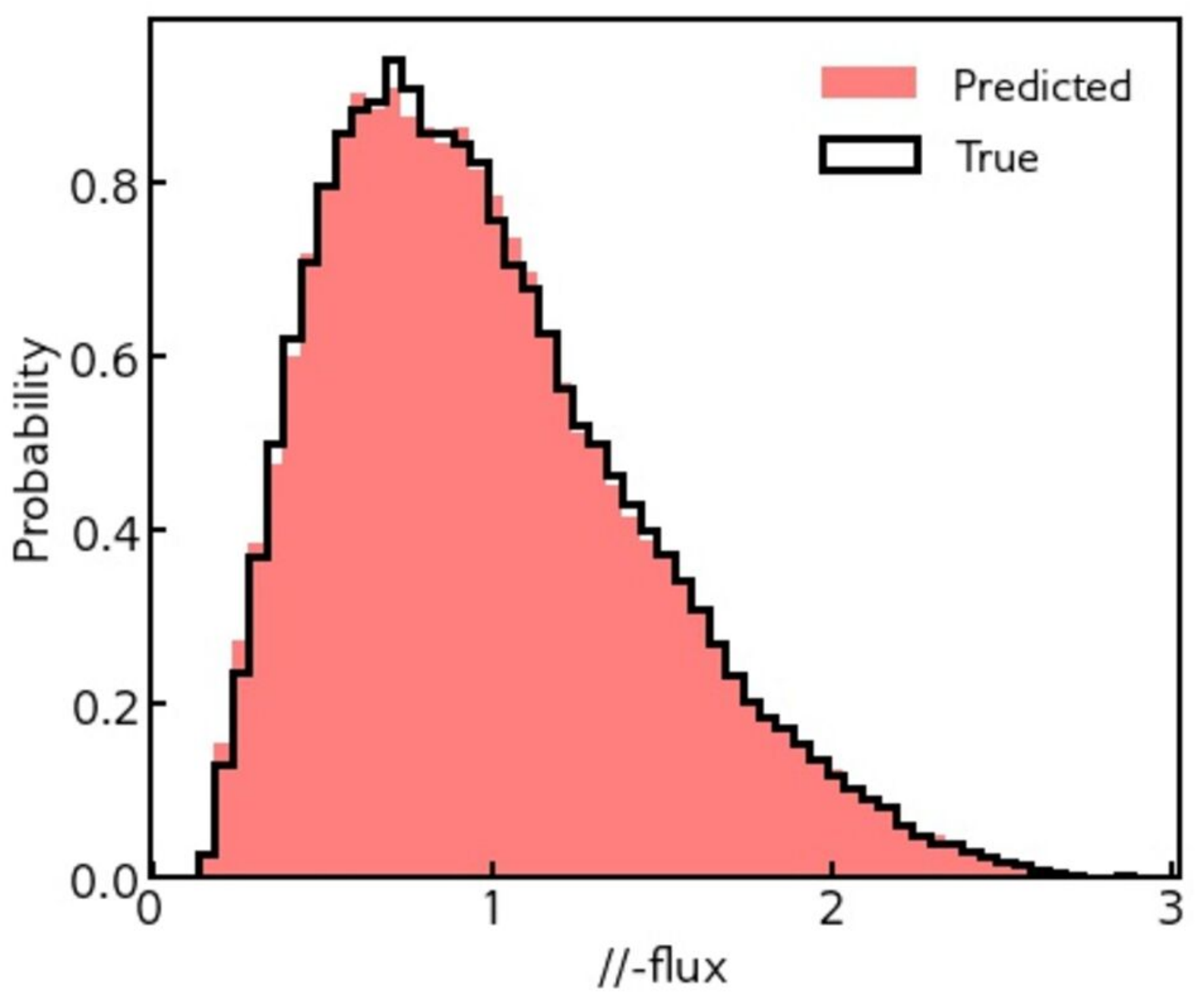

哭畺 


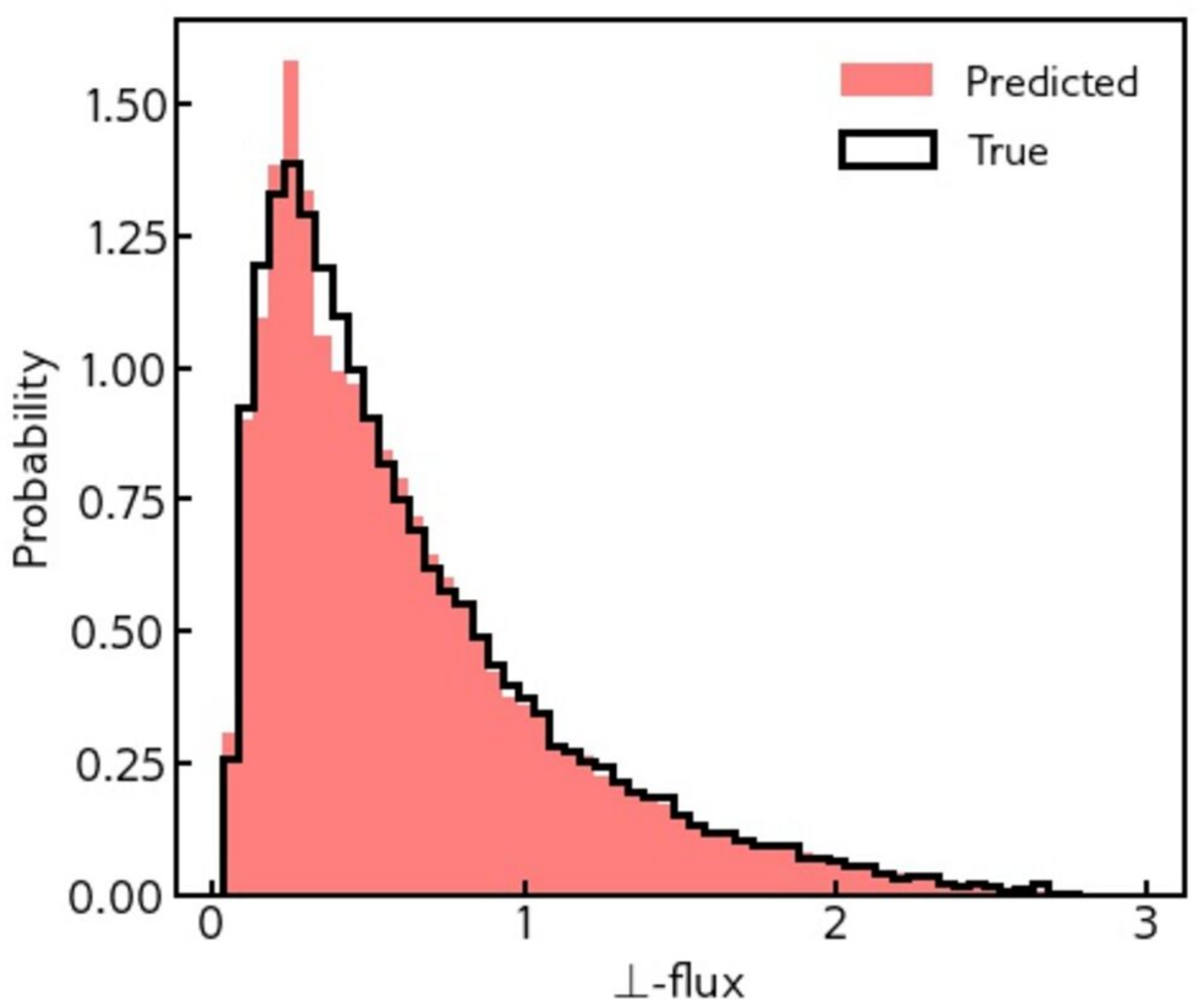

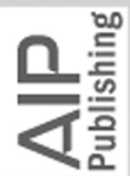




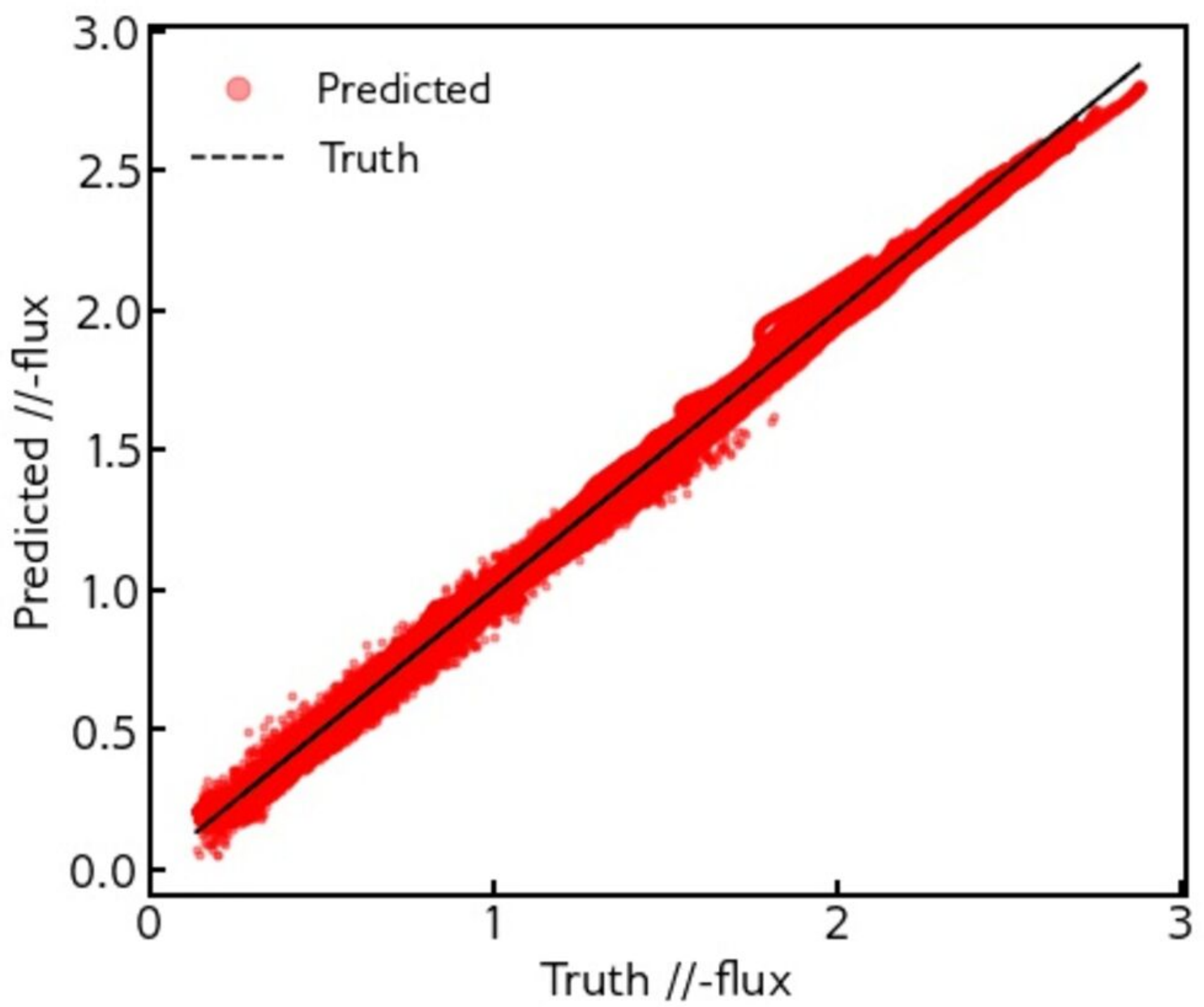

兄耪言 


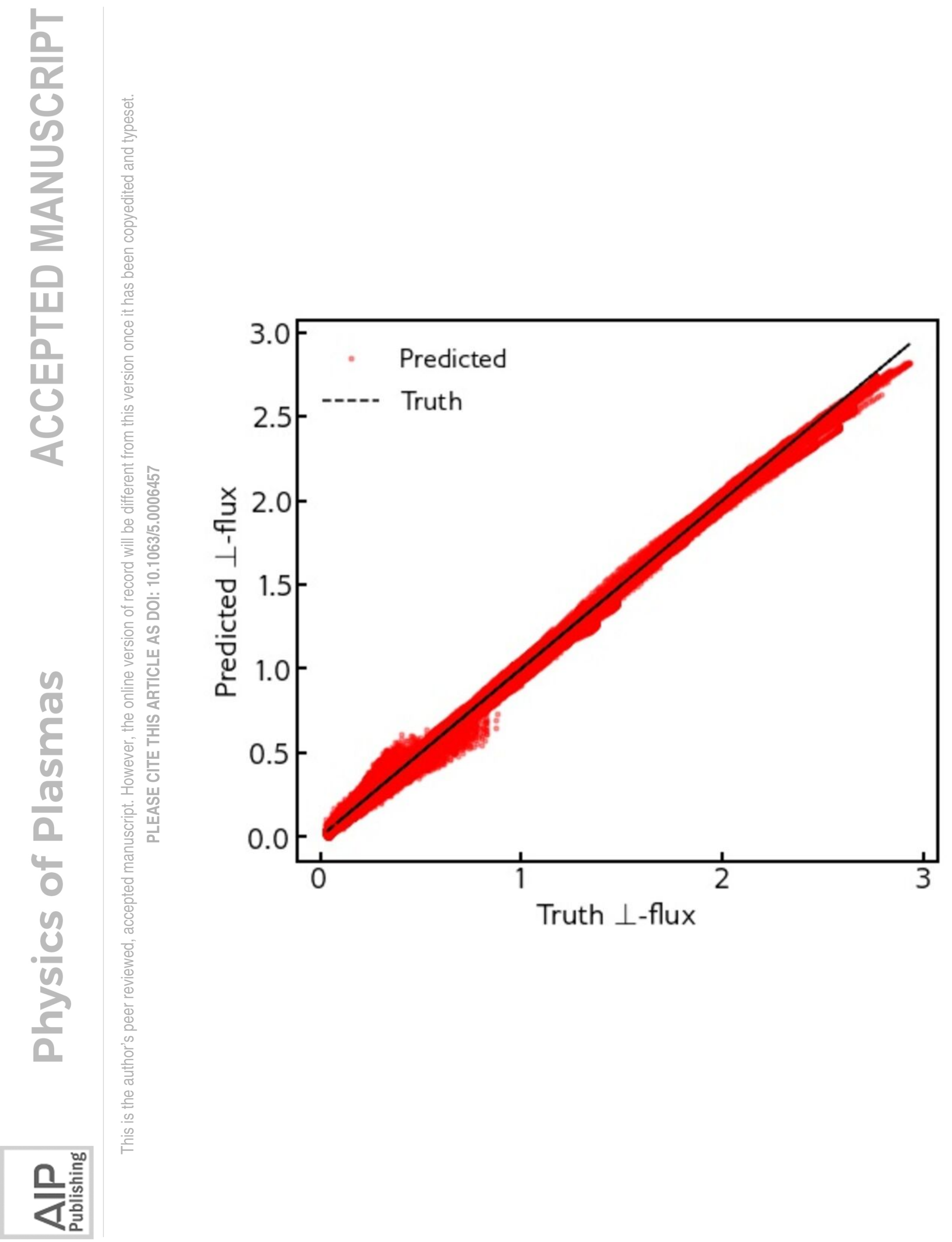




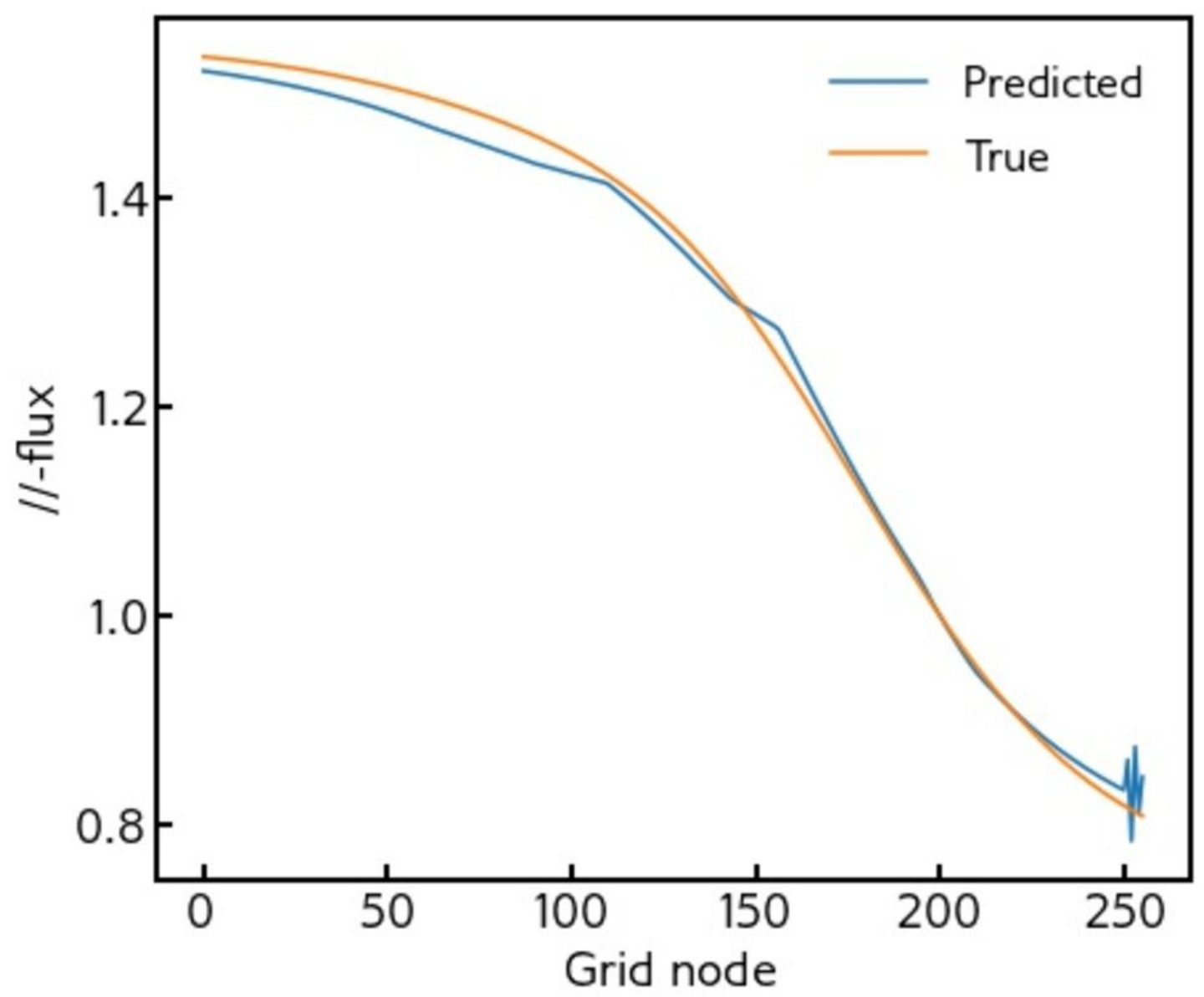

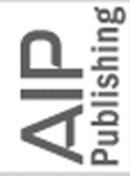




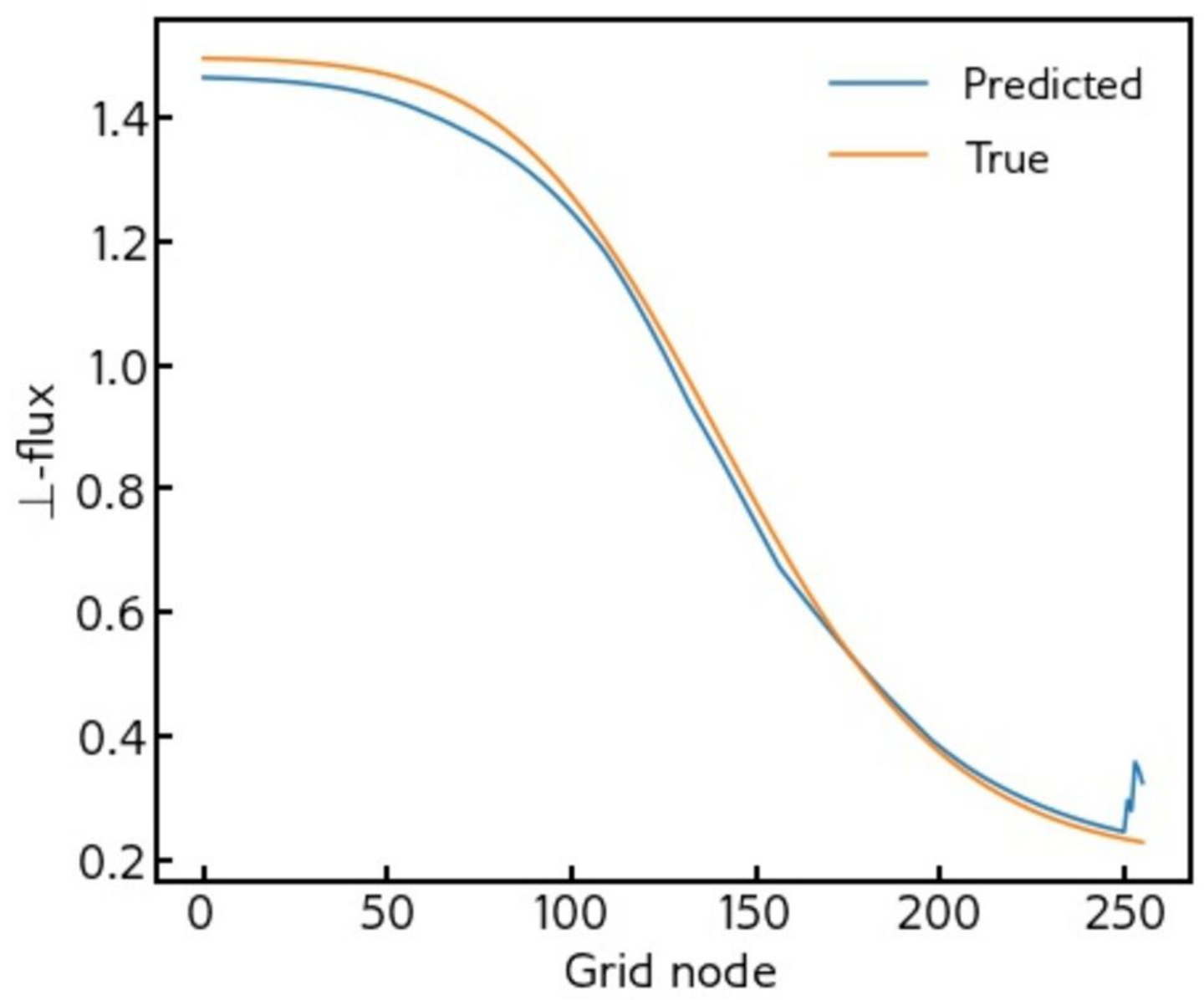

은 


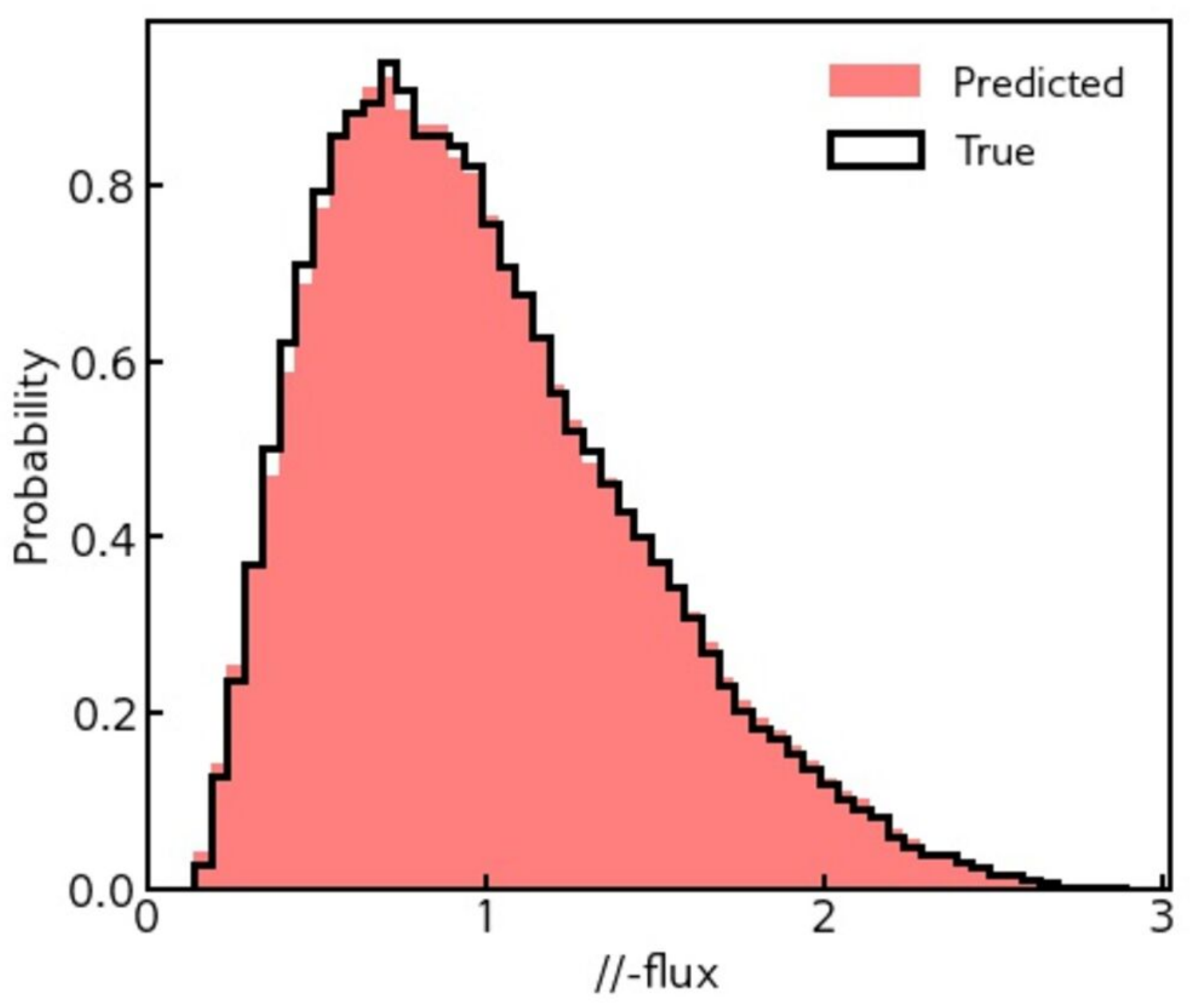

哭畺 


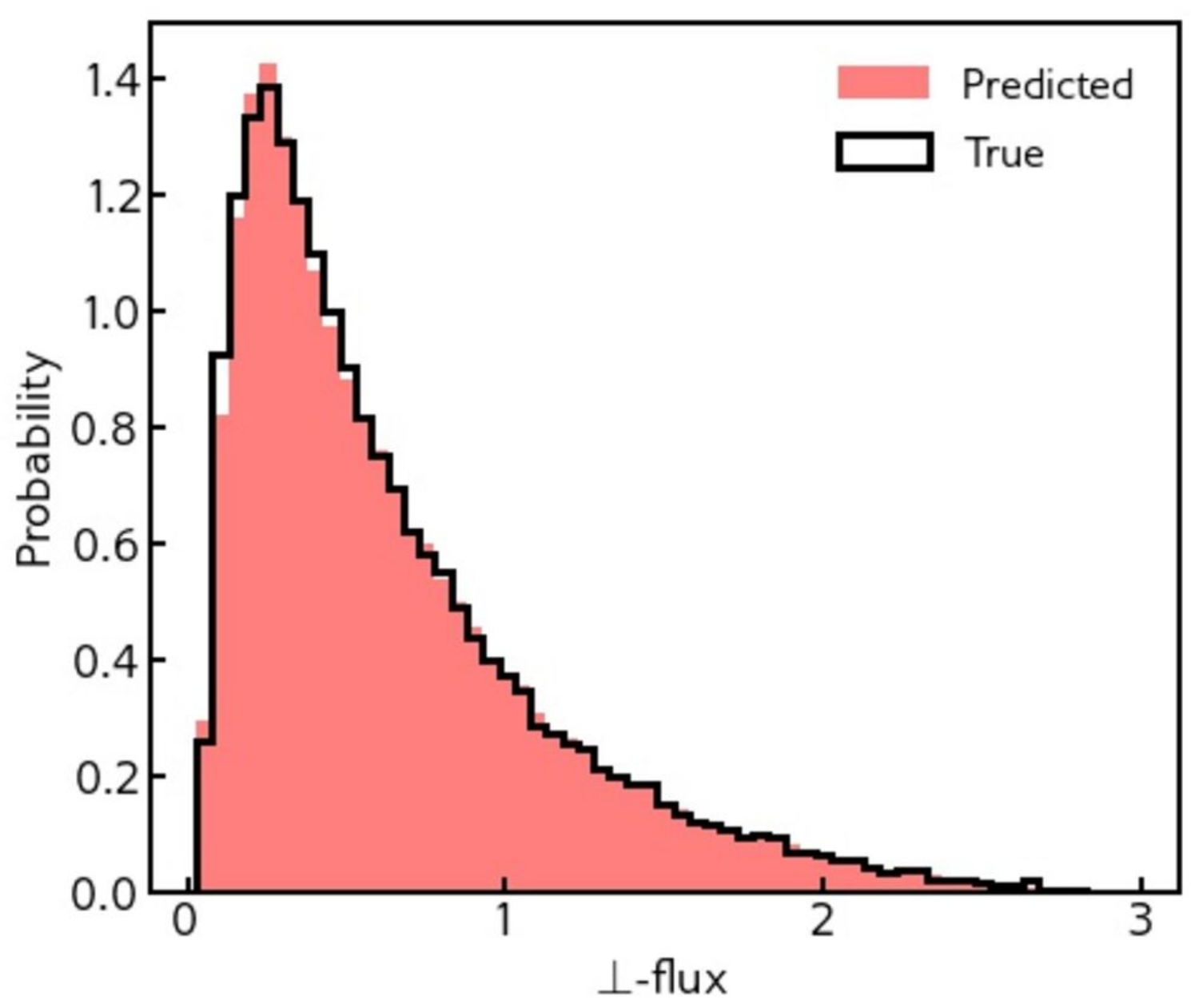

는 


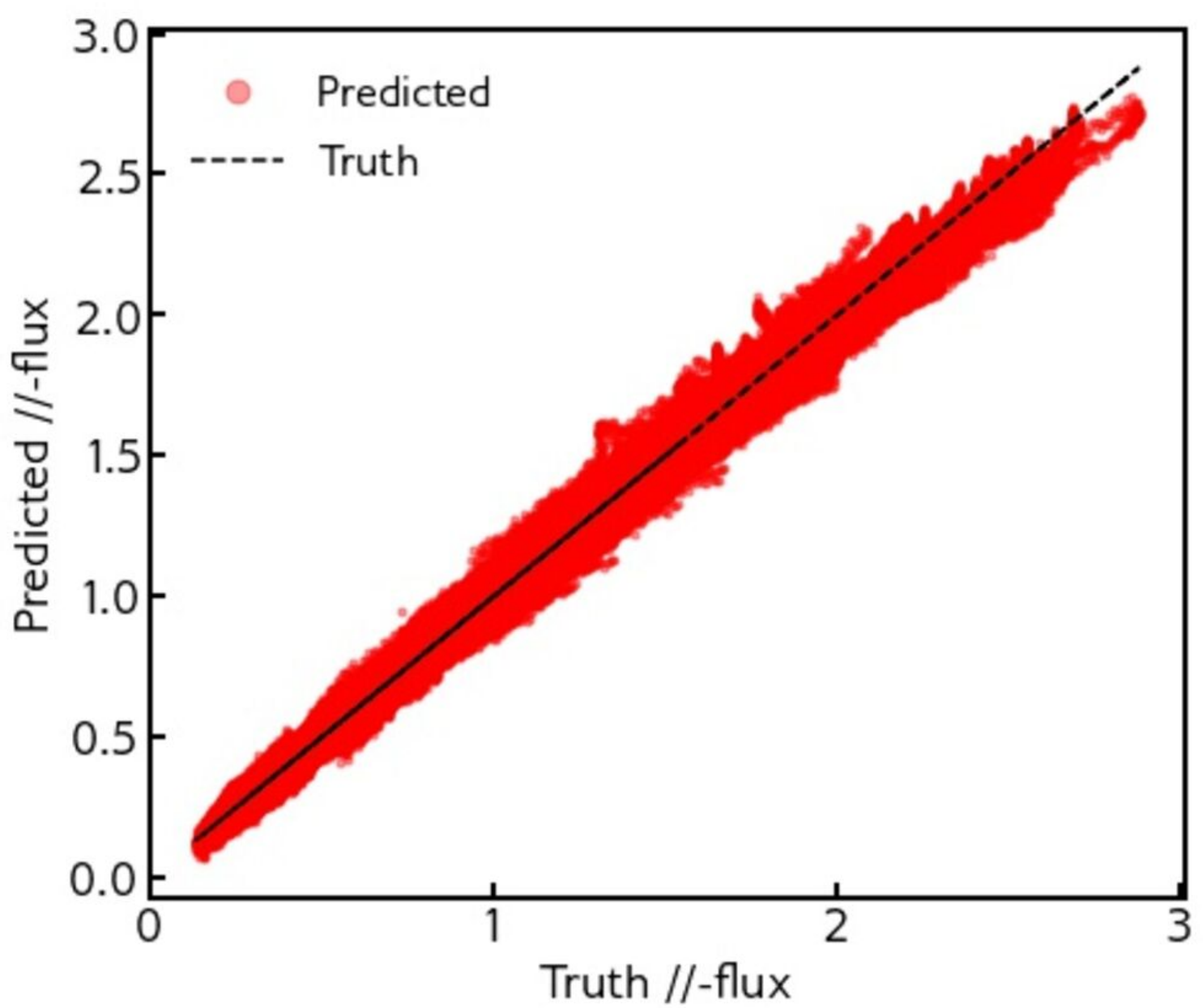

兄耪言 


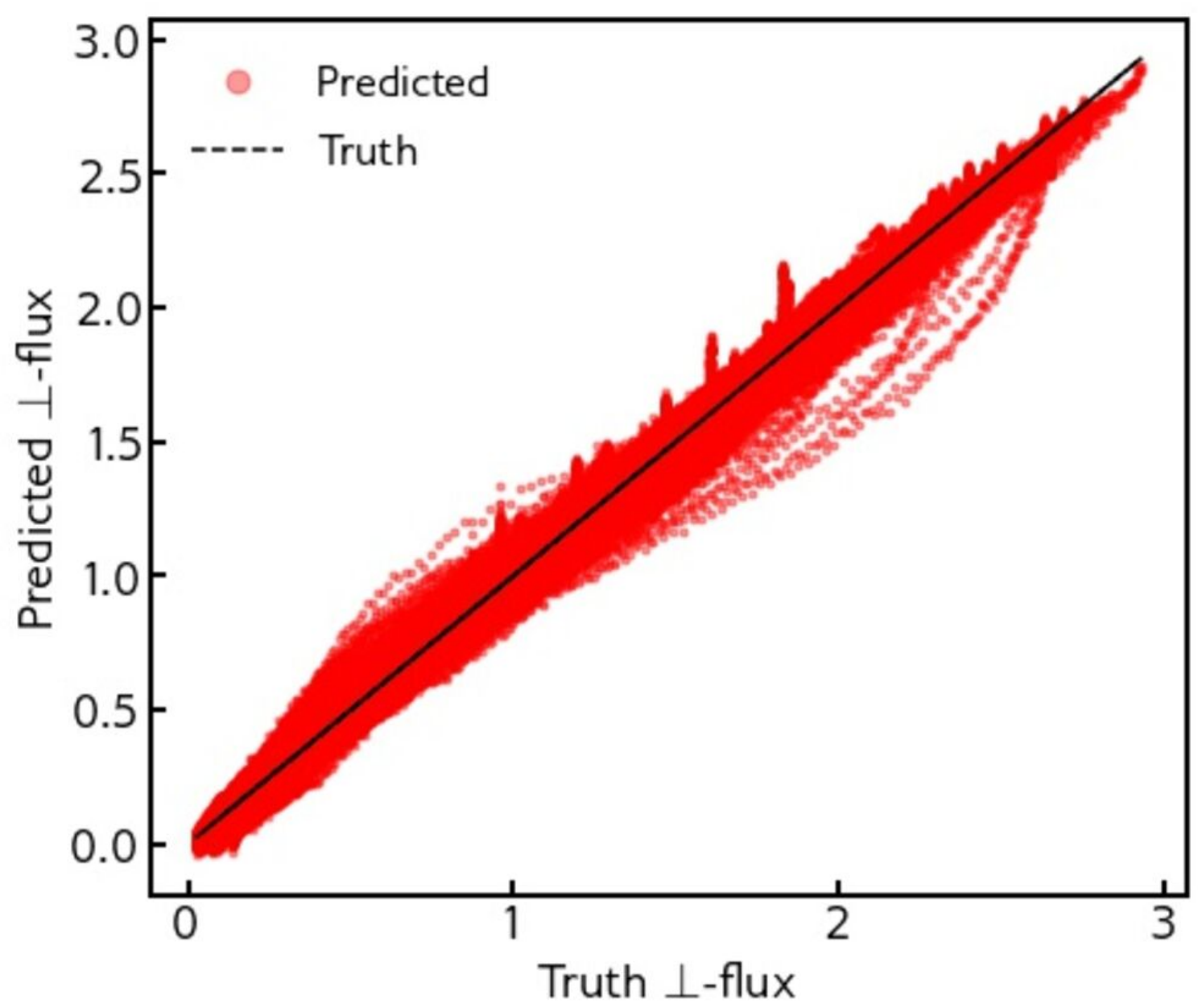

兄耪言 


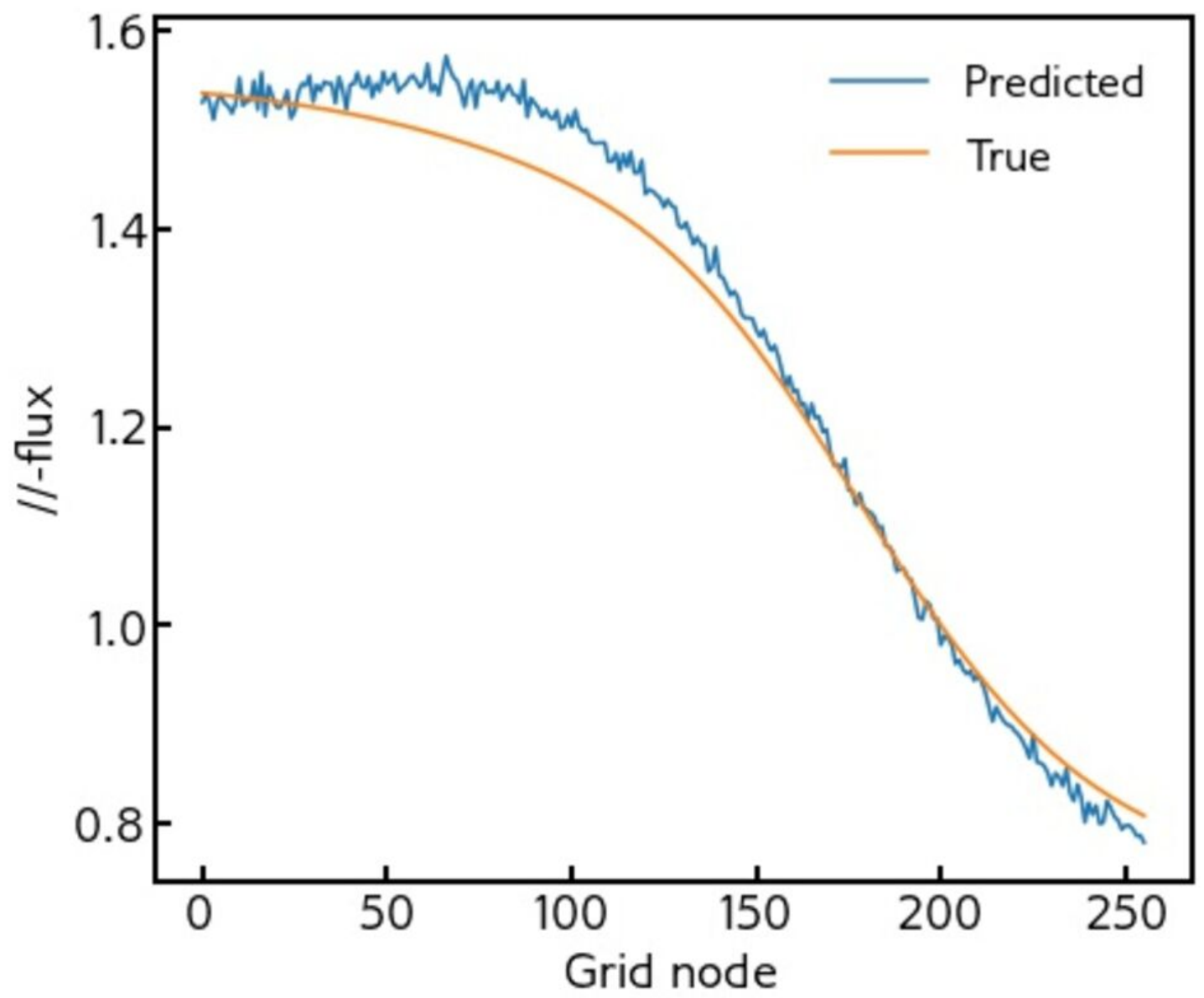

碞衰 


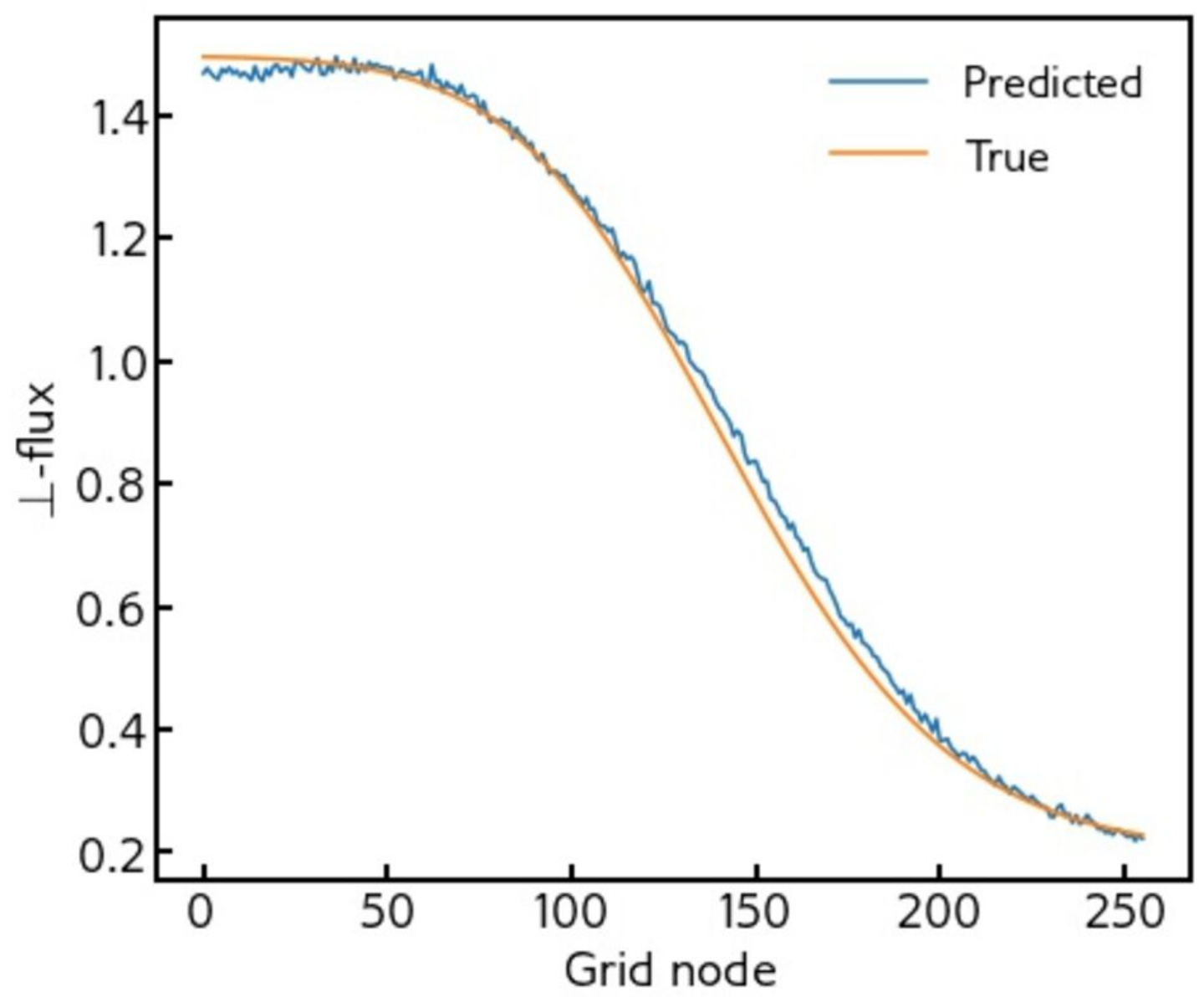

은 


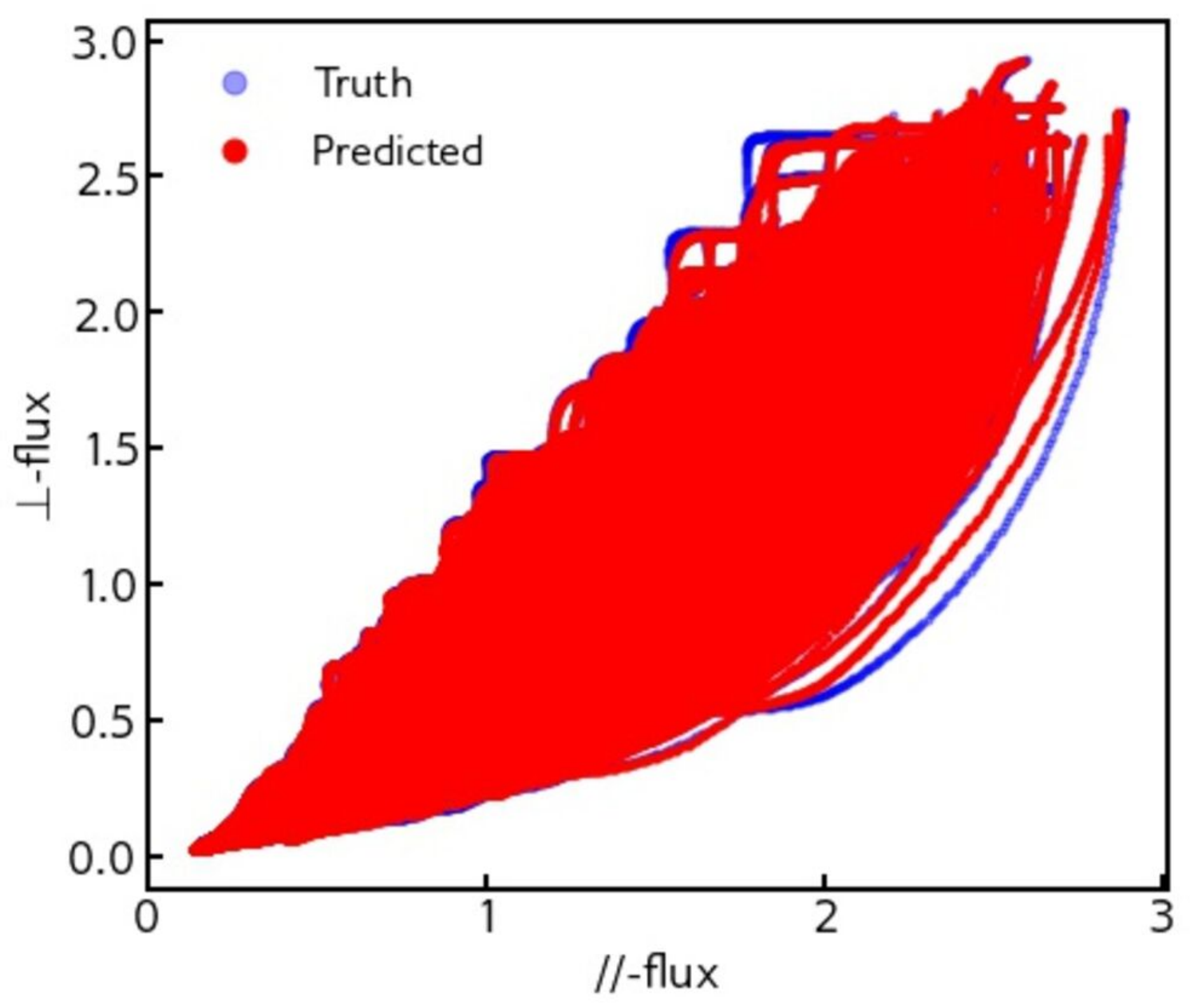

兄耪言 


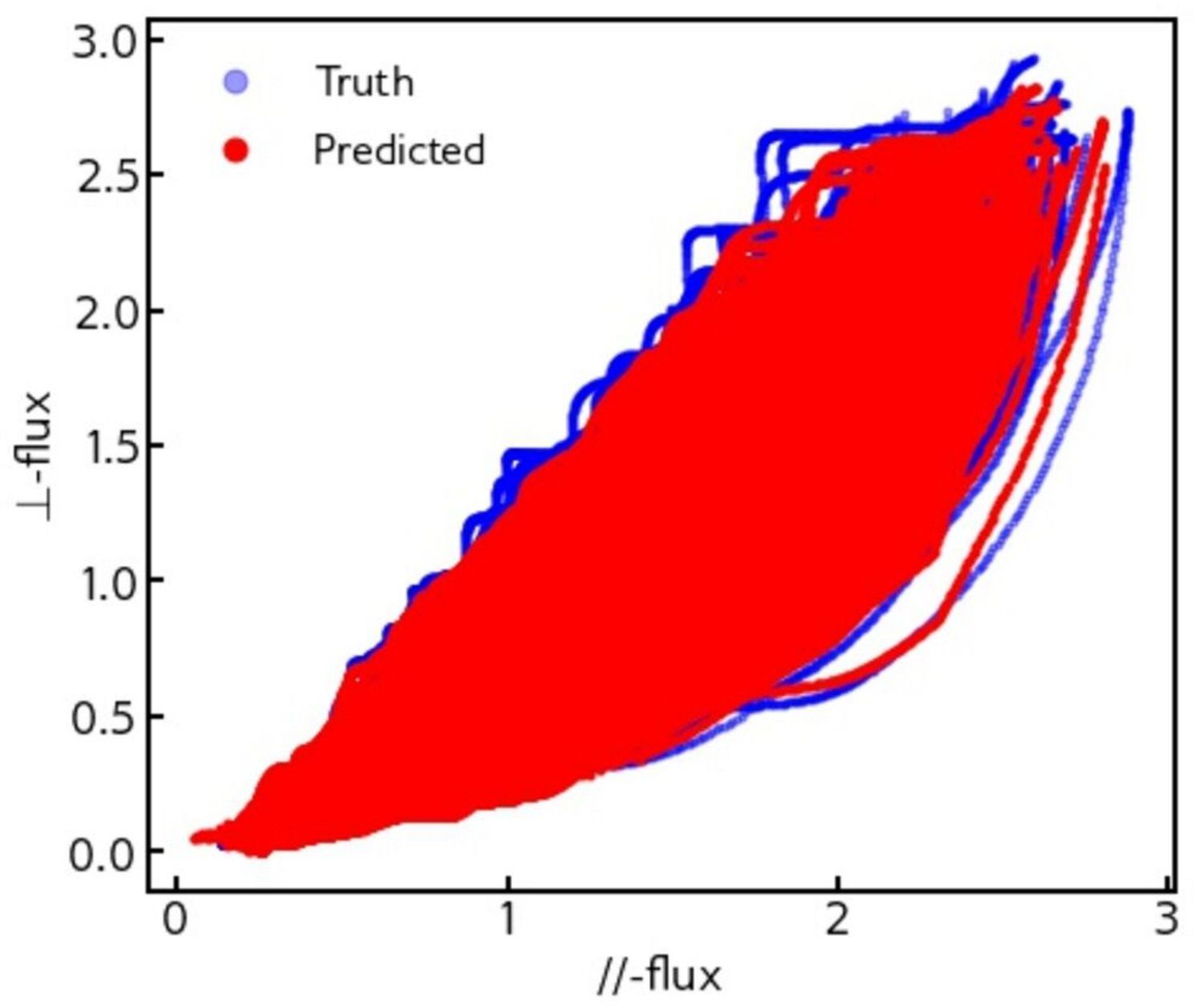

兄耪言 


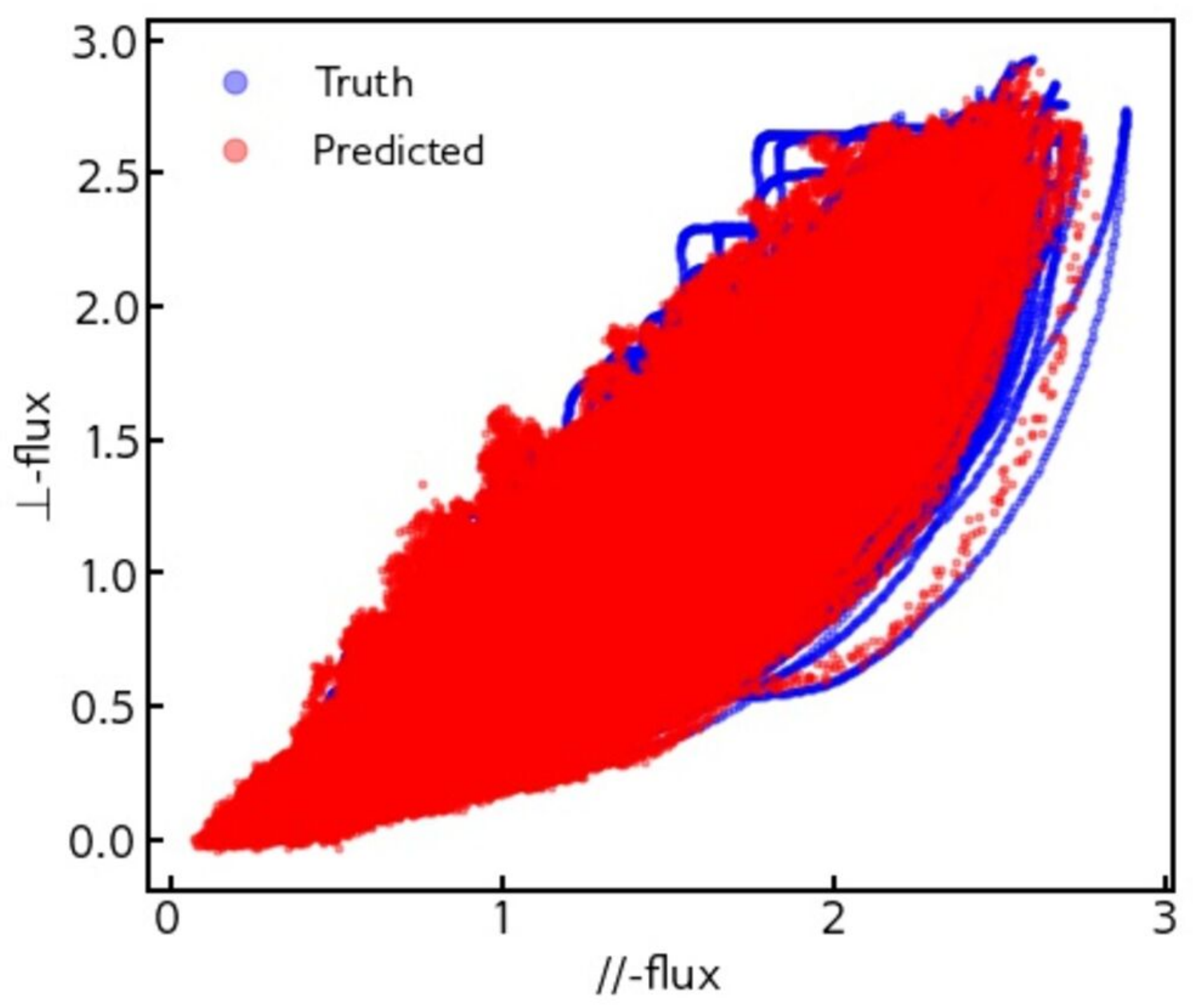

兄耪言 


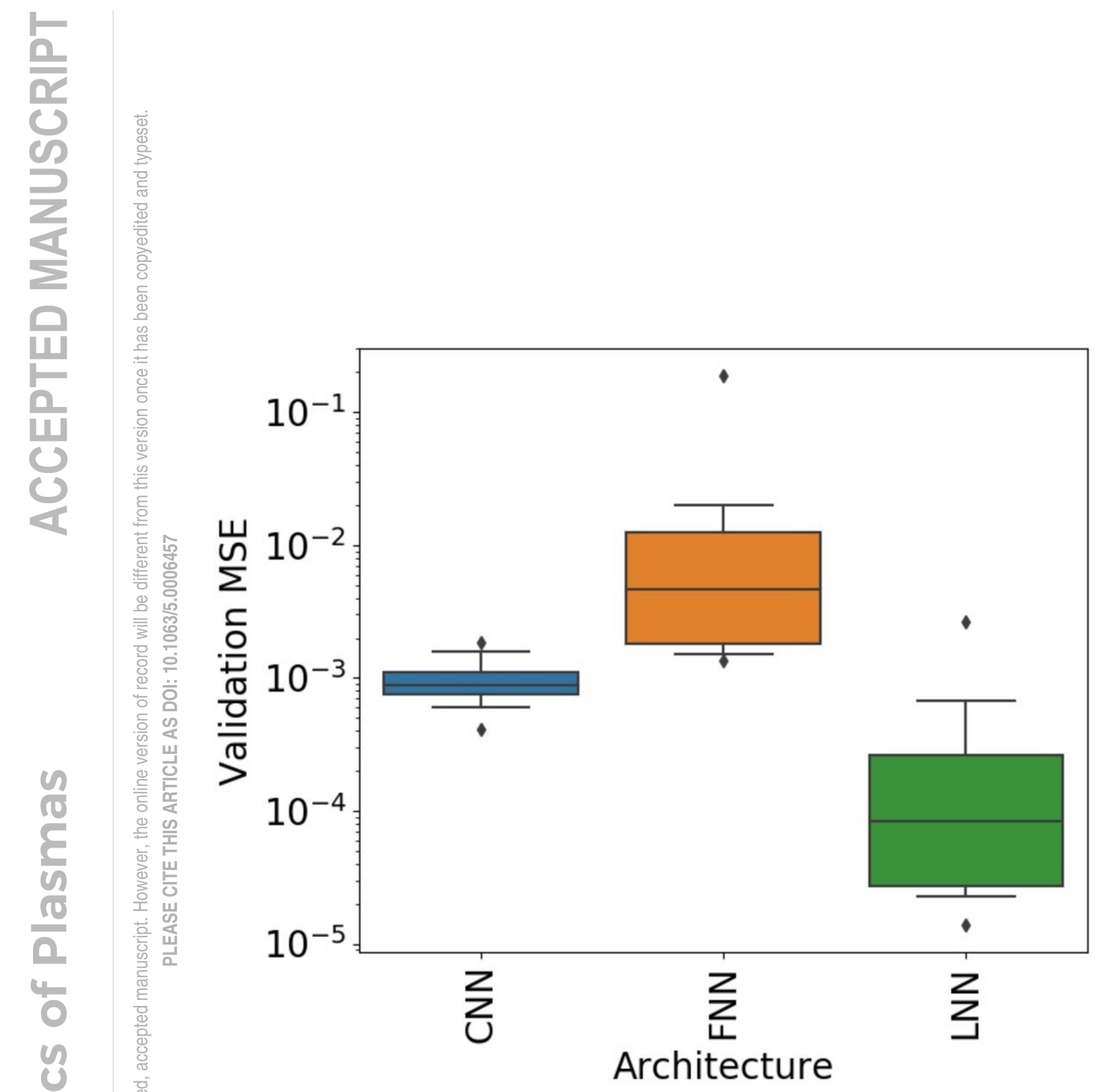

兄旁高 


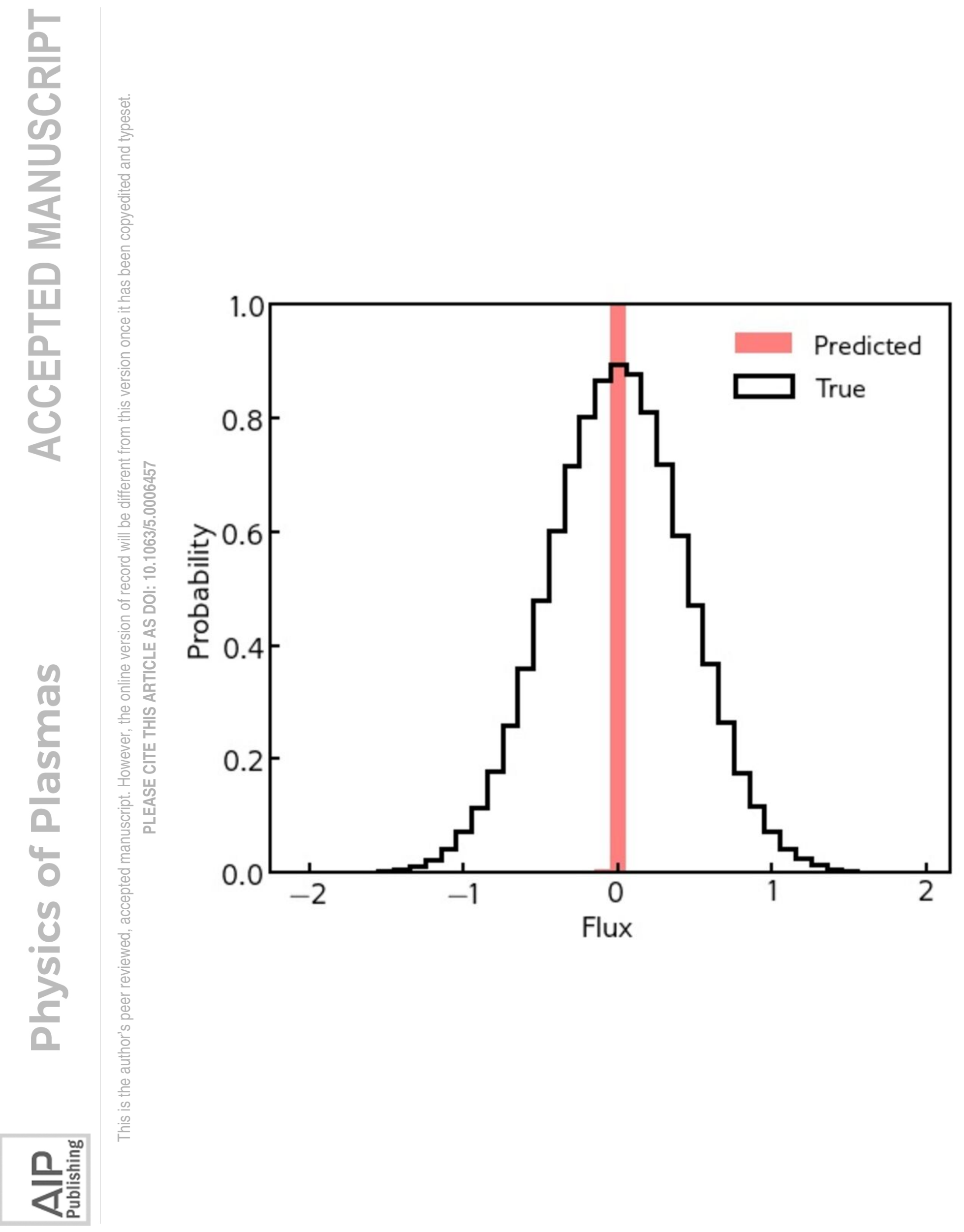




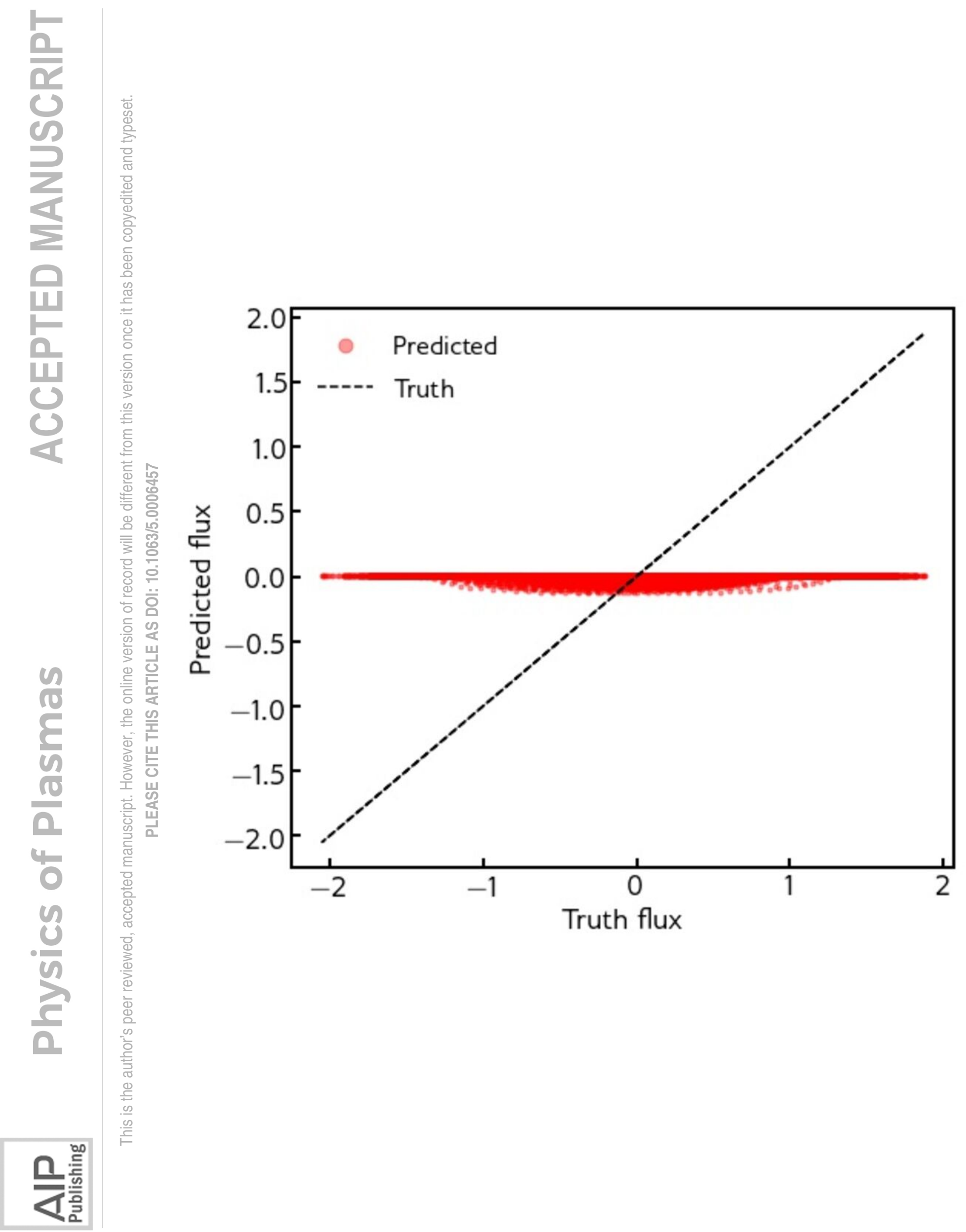




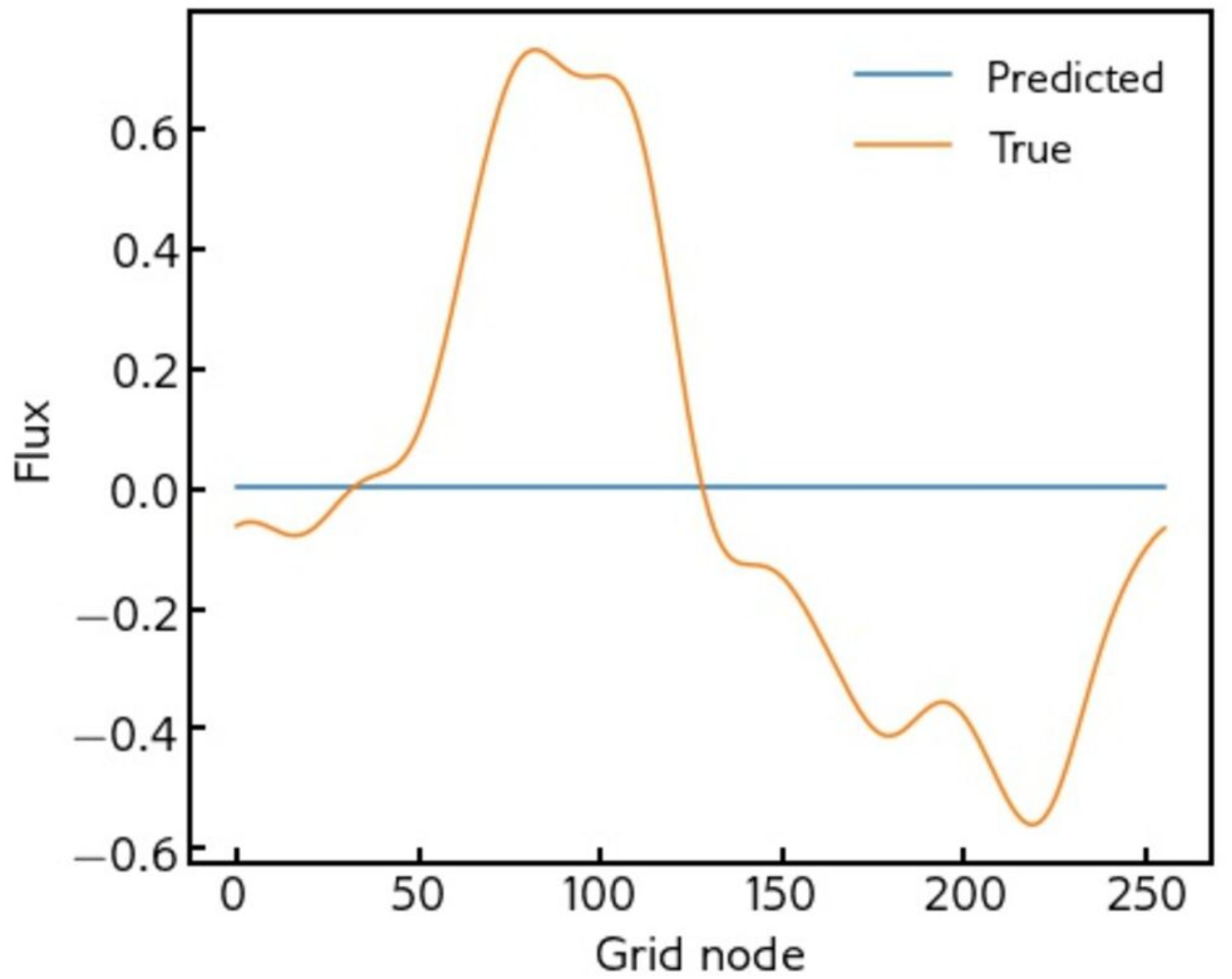

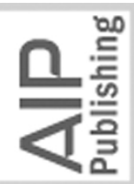




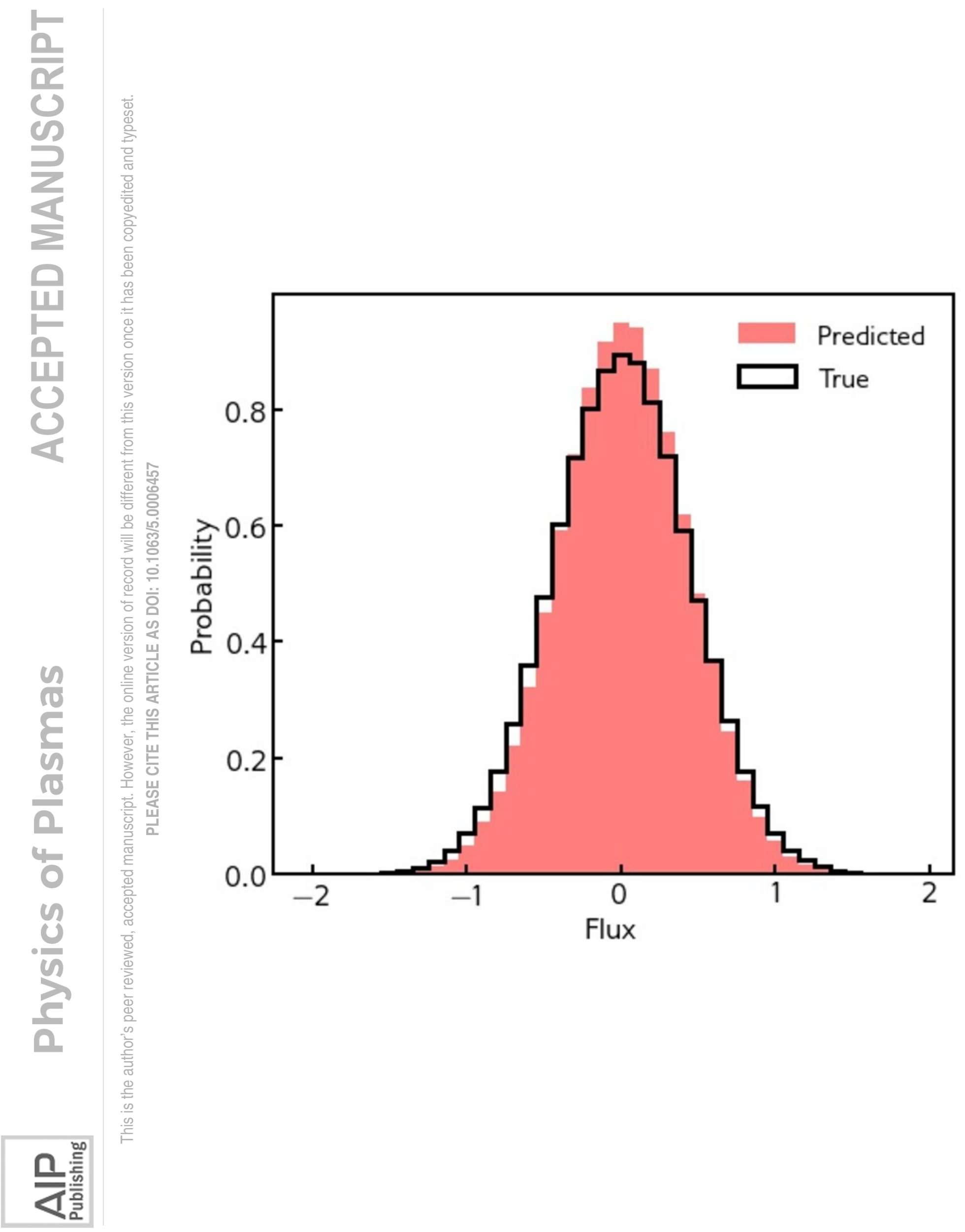




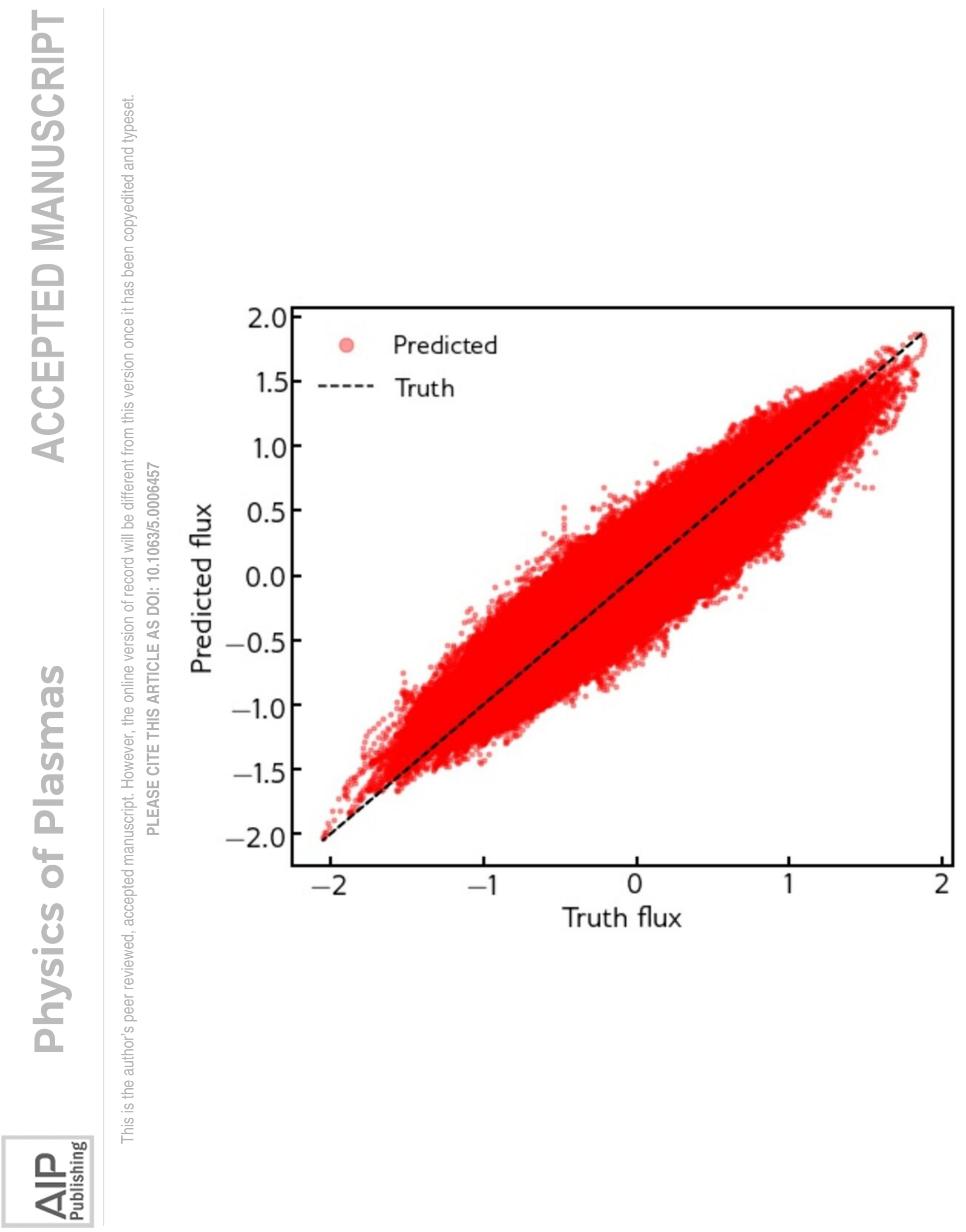




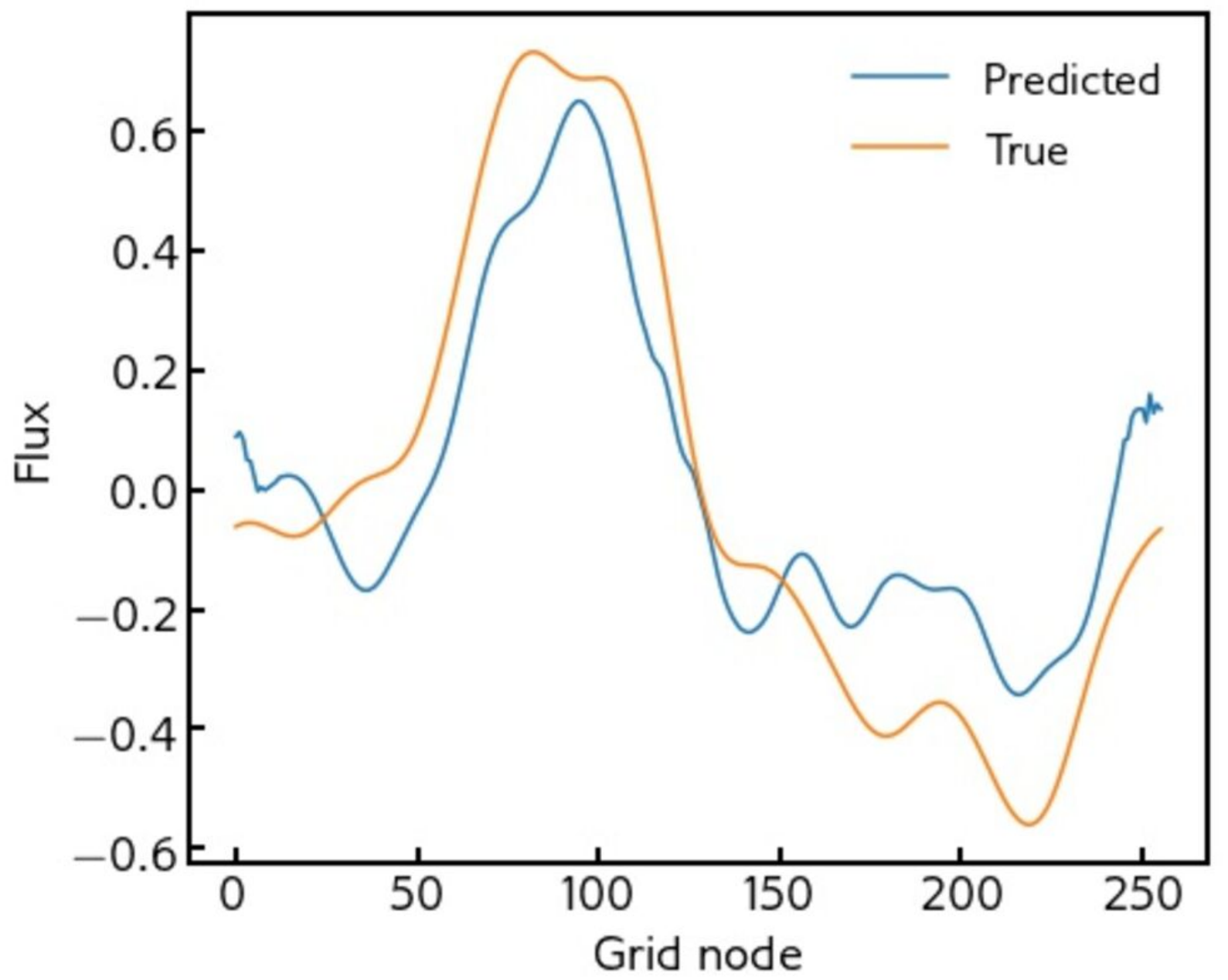

는 


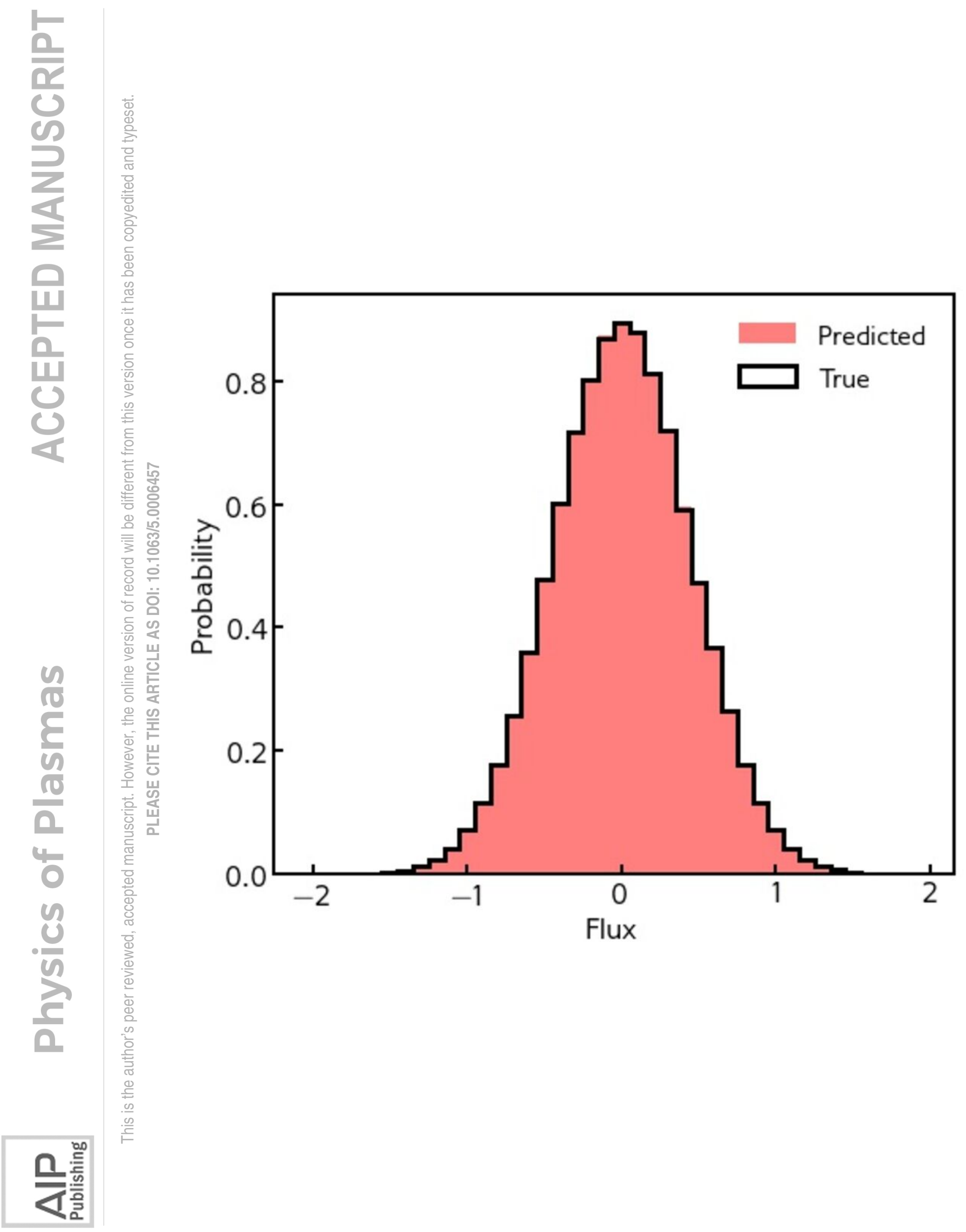




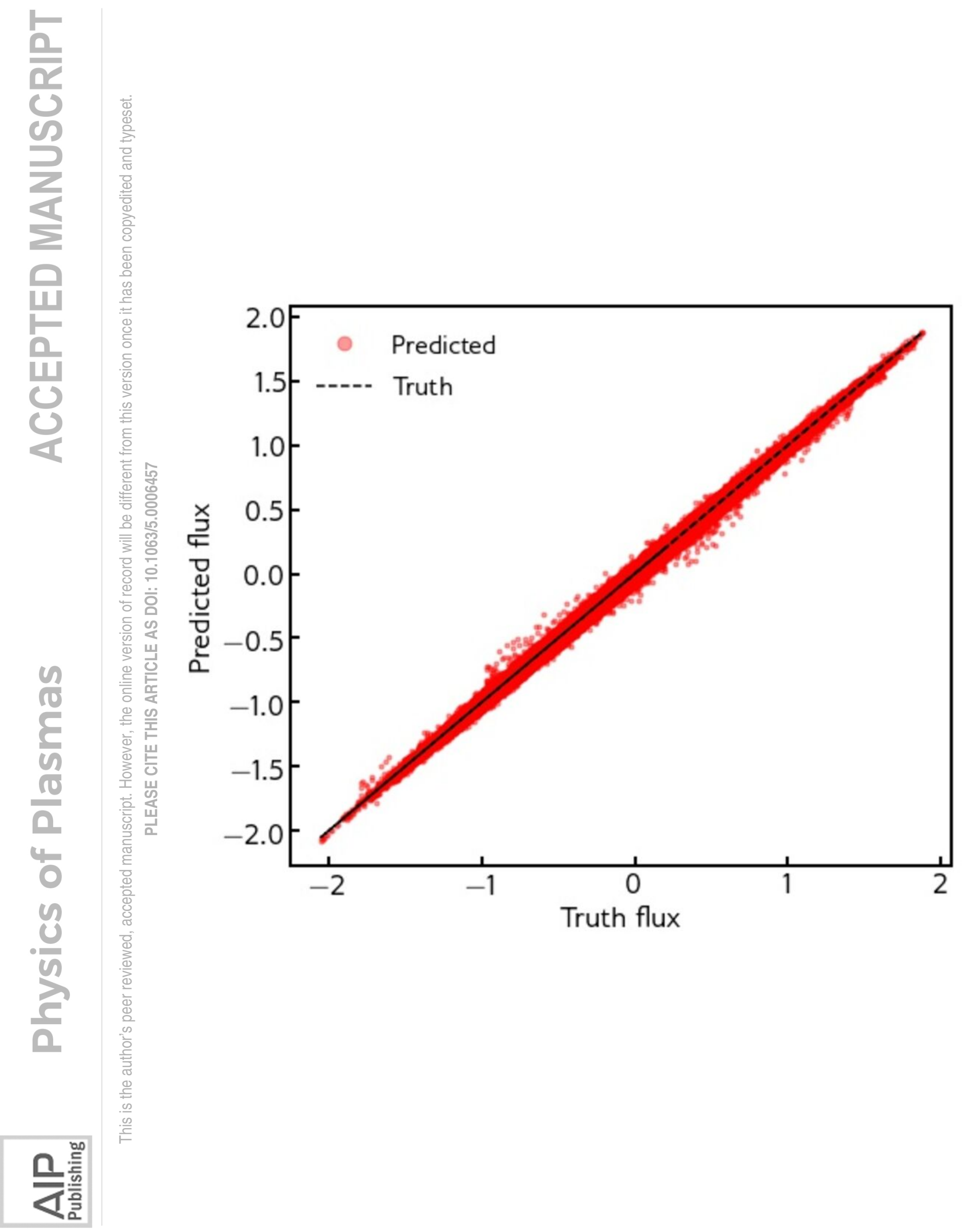




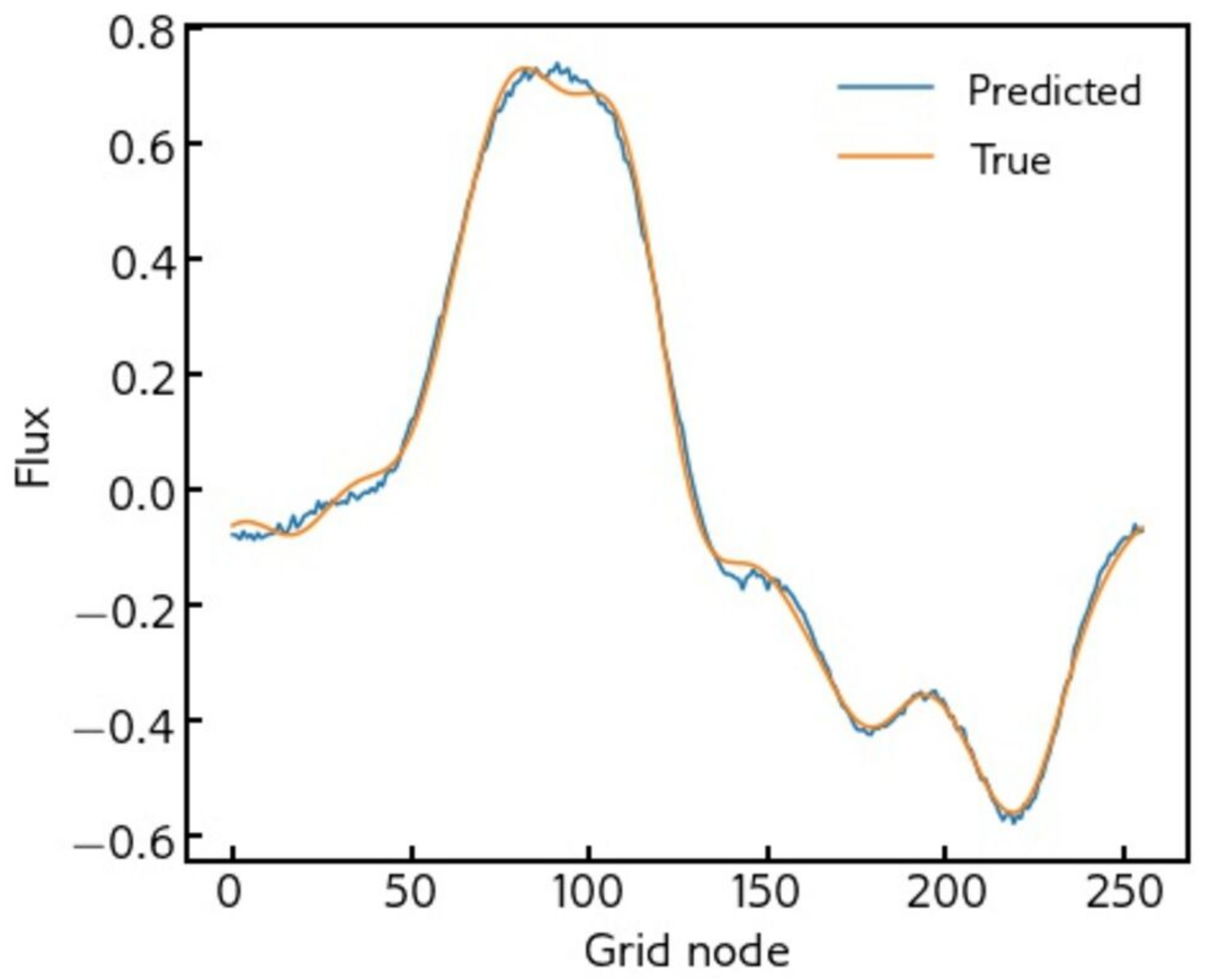

은 


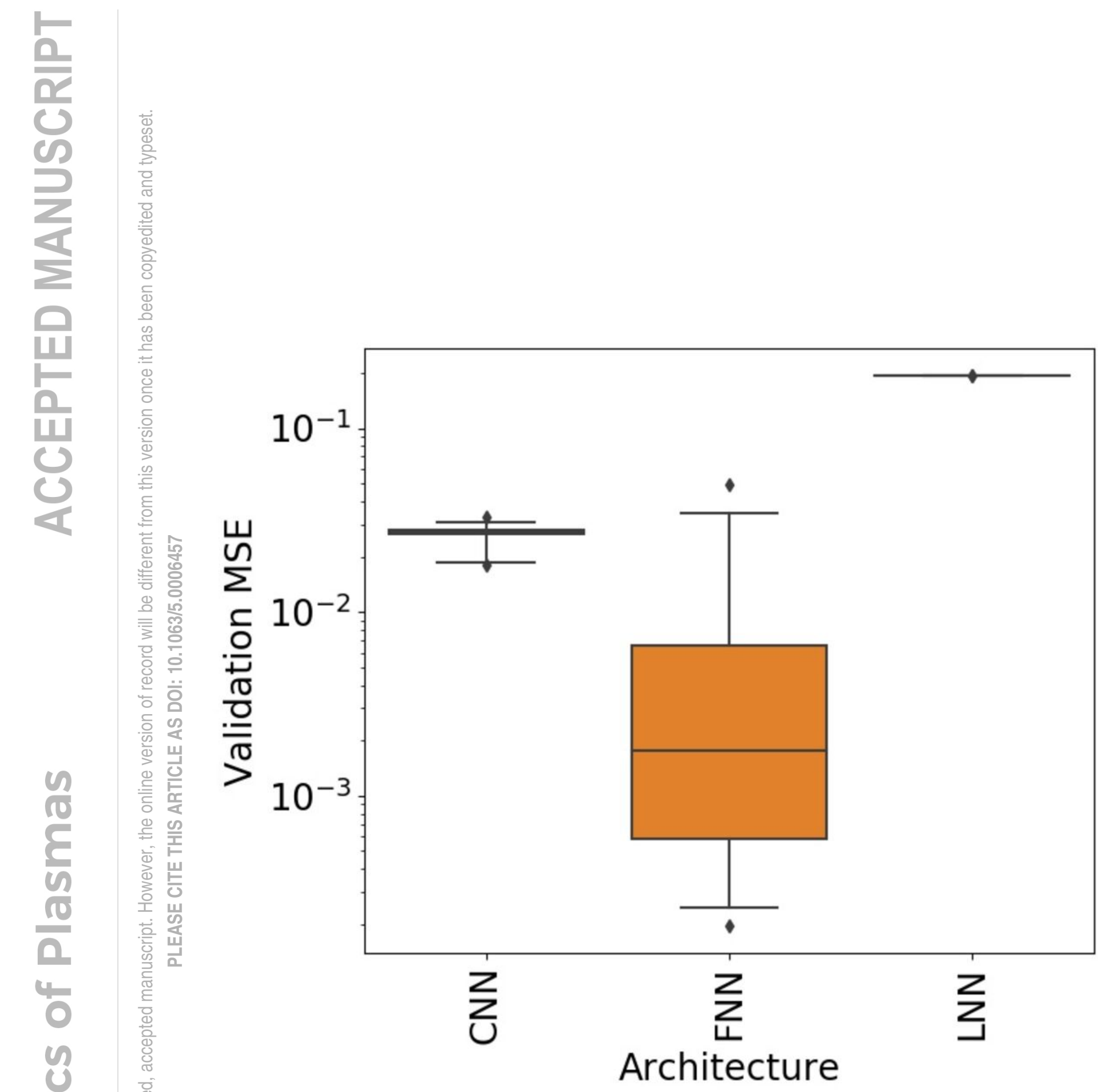

兄旁高 


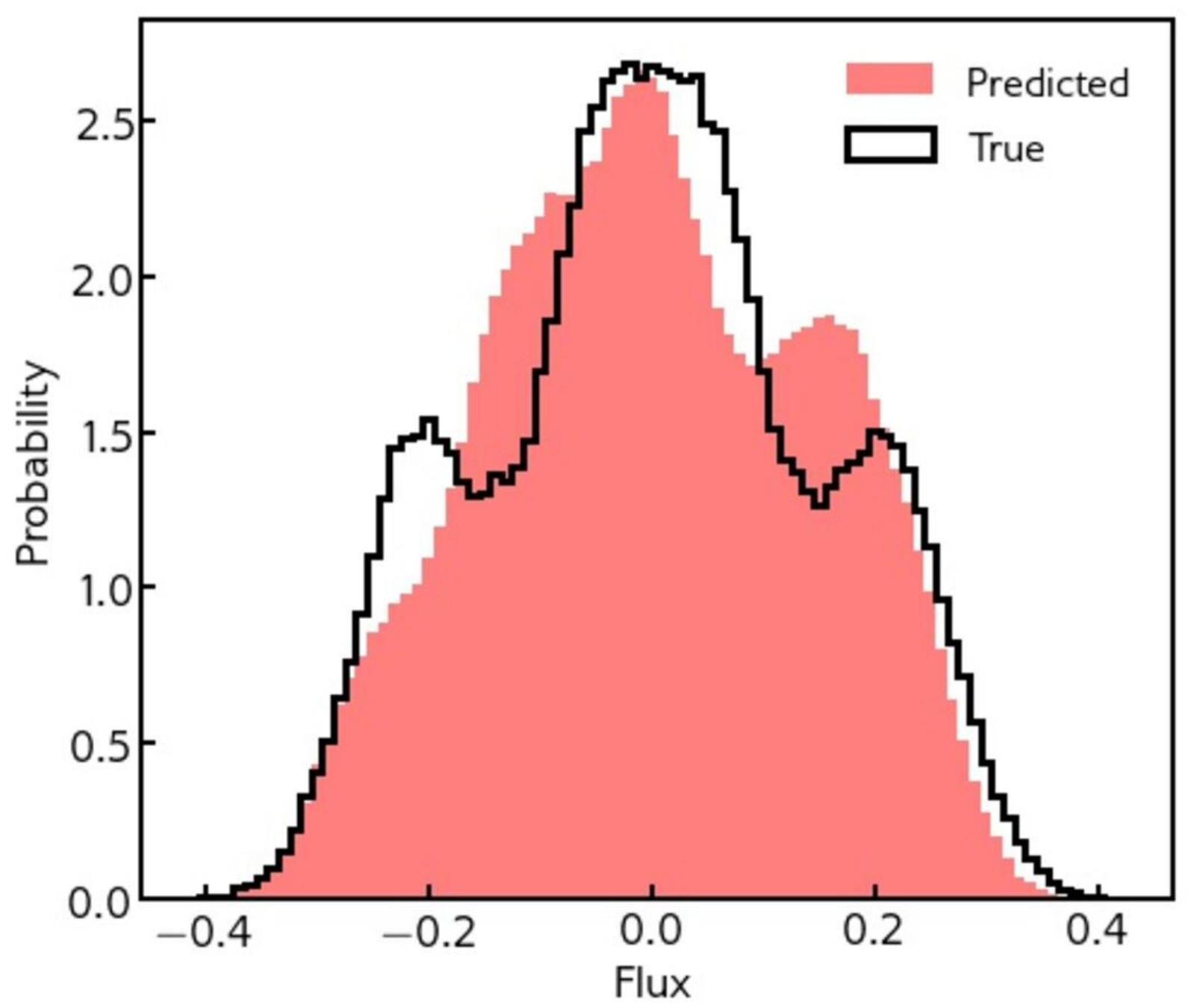

은 


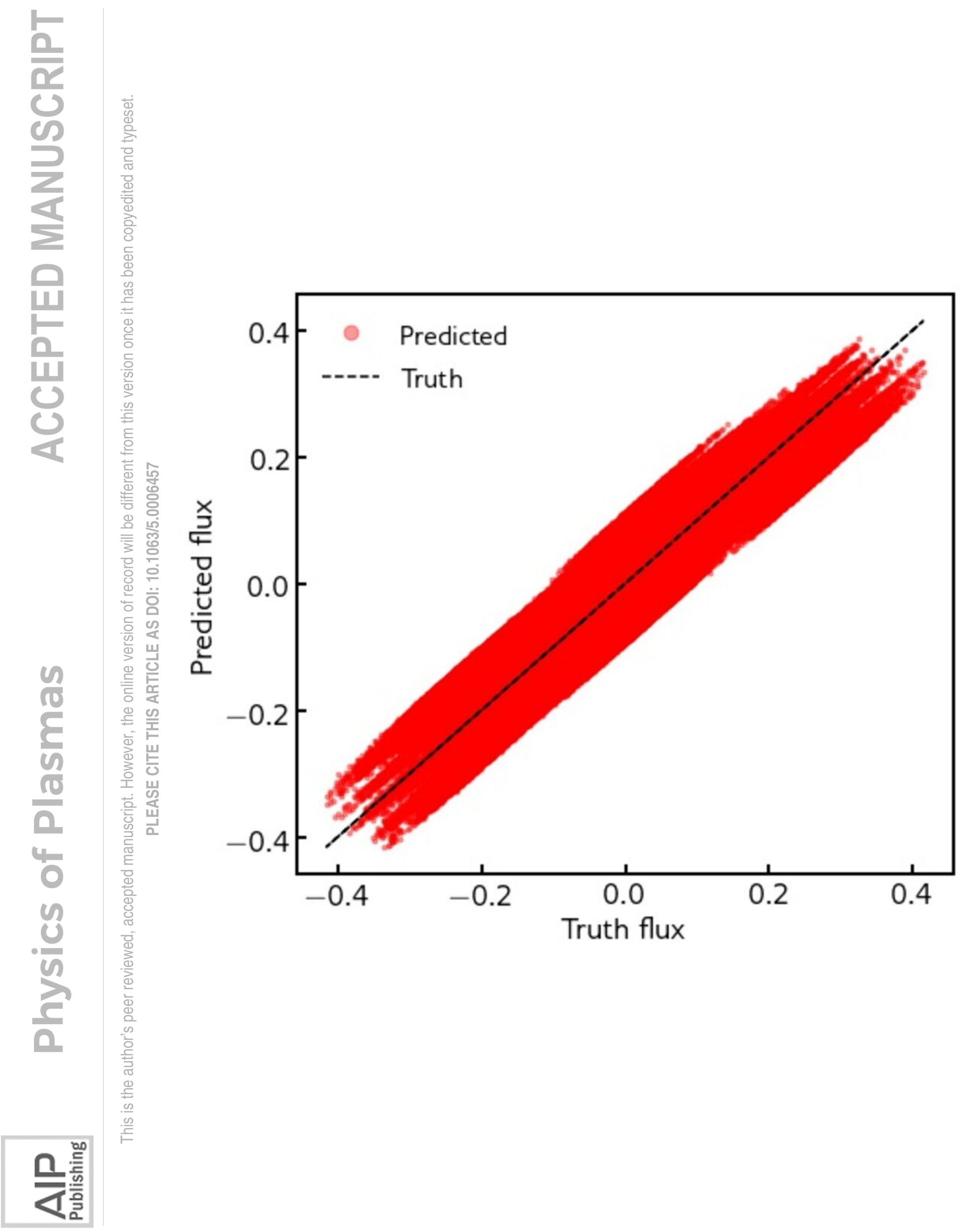




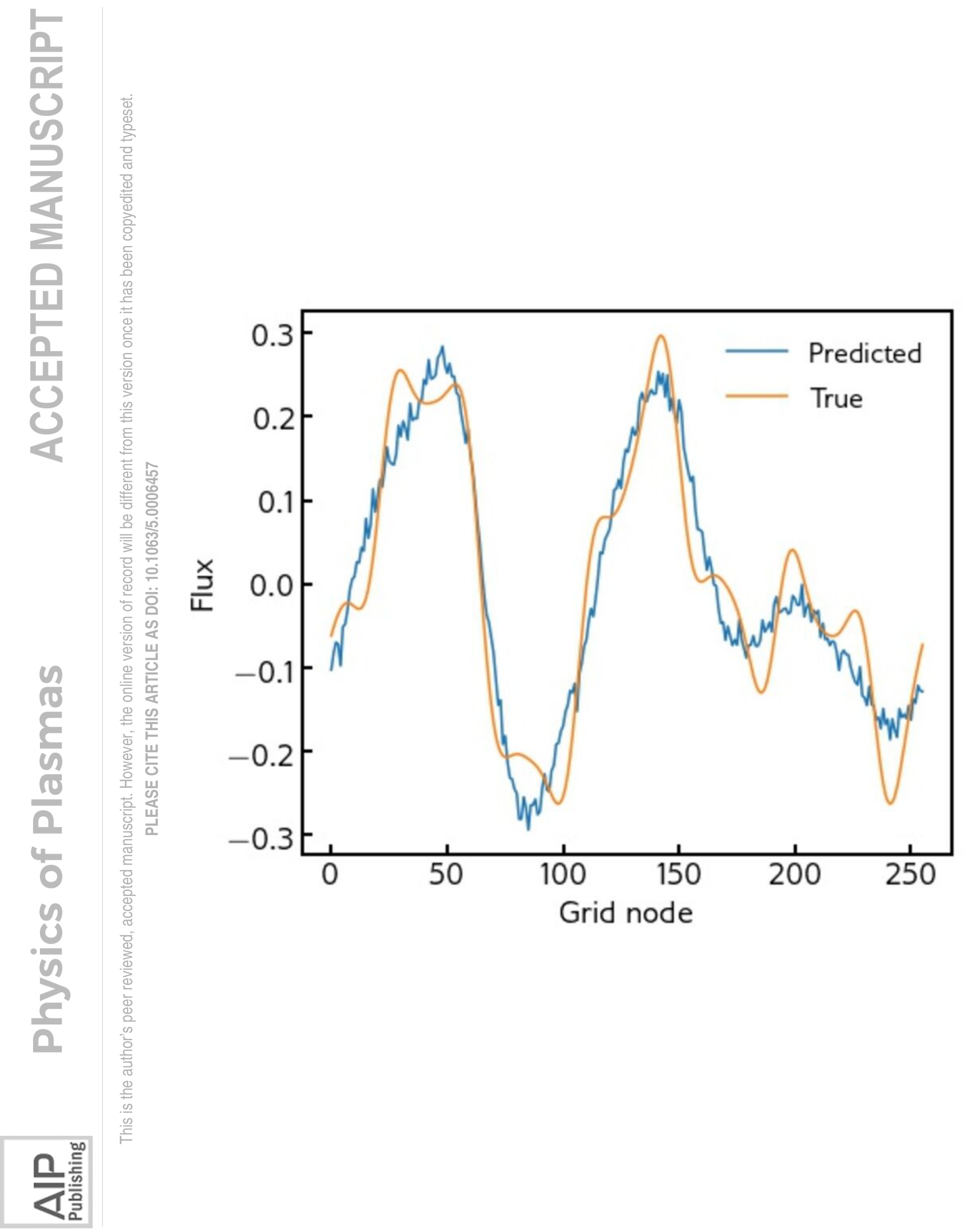




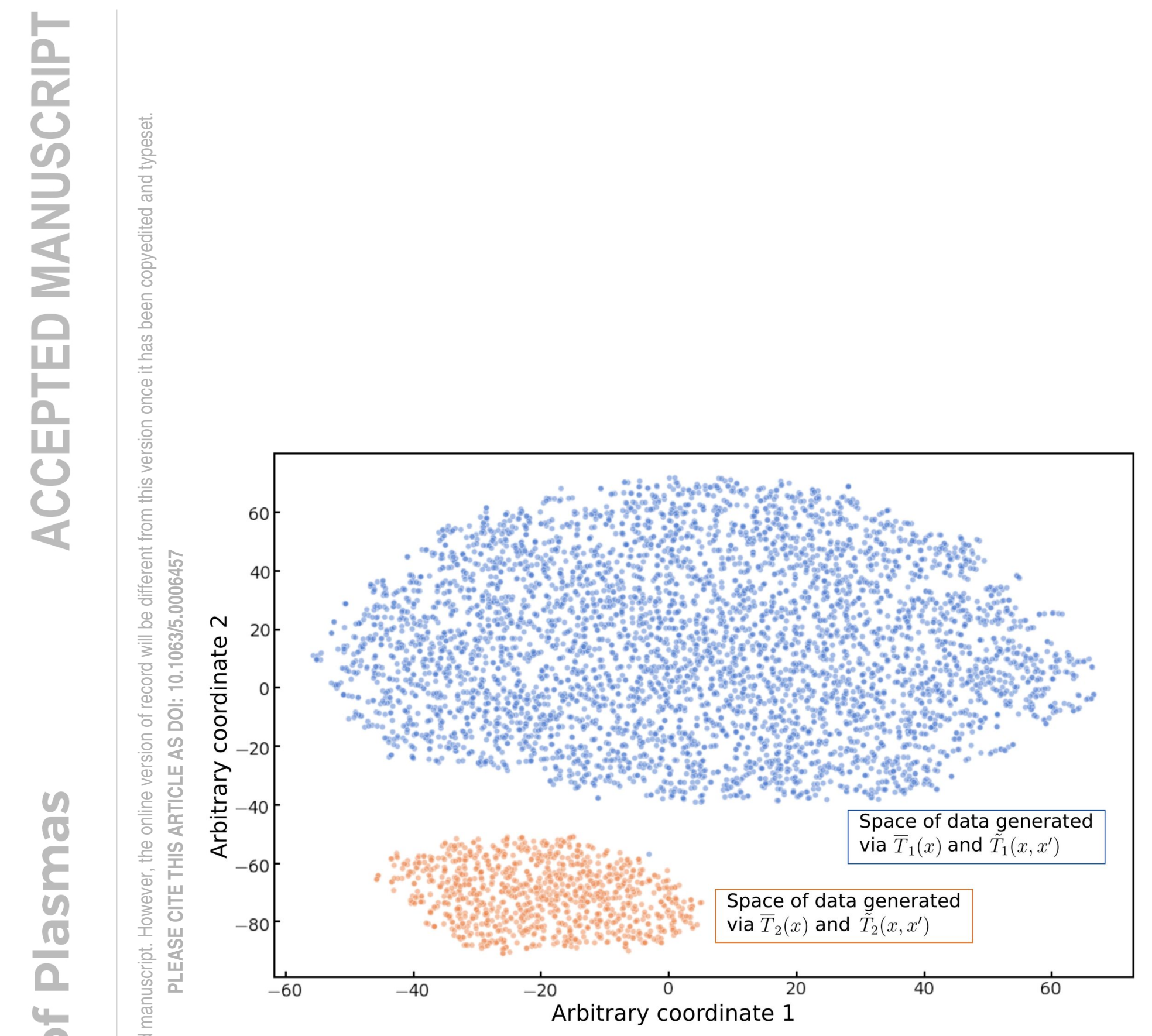




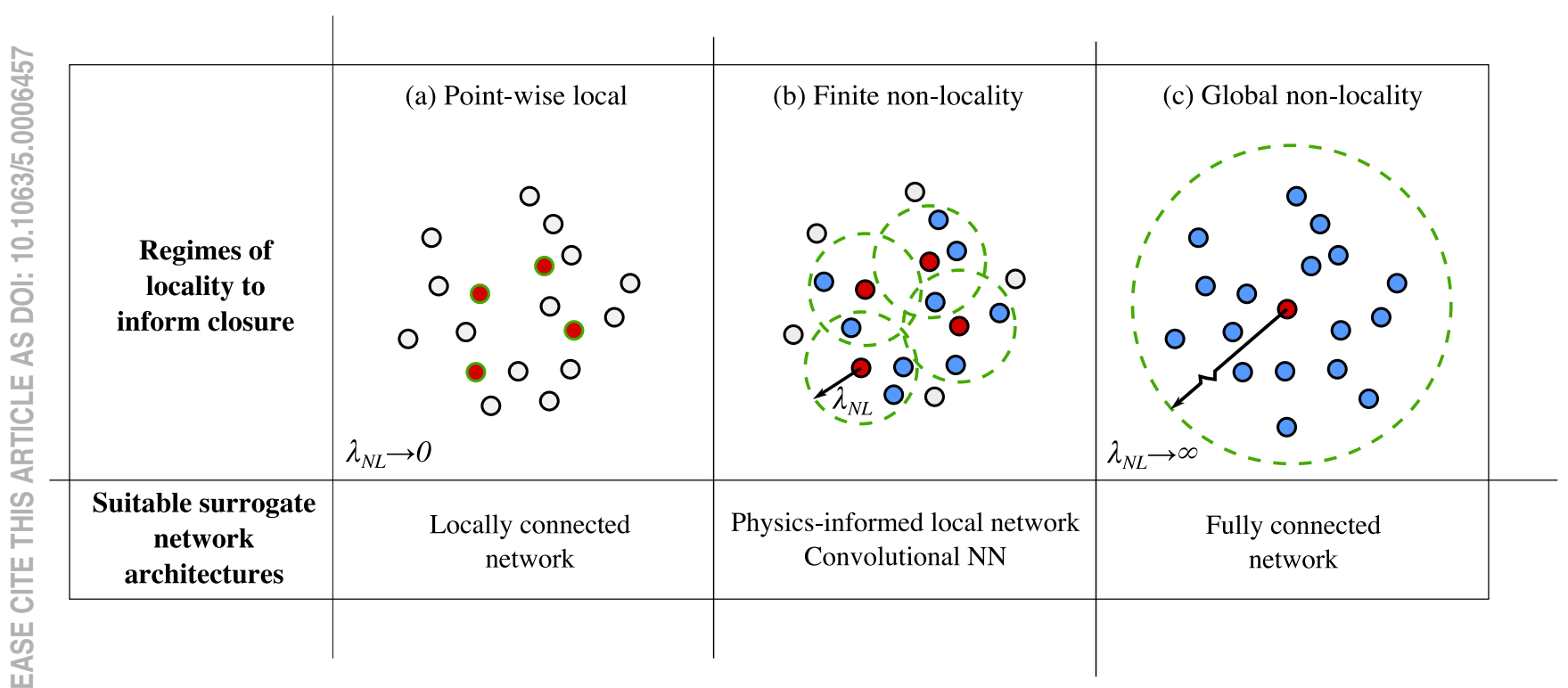

